Explosive Micro-Bubble Actuator

Dennis van den Broek 
The research described in this thesis was carried out at the Transducers Science and Technology Group of the MESA+ Research Institute at the University of Twente, Enschede, The Netherlands. The project was financially supported by the Dutch Technology Foundation (STW).

Promotiecommissie:

Voorzitter

prof. dr. ir. J. van Amerongen

Secretaris

prof. dr. ir. A.J. Mouthaan

Promotor

prof. dr. M.C. Elwenspoek

Referent

prof. dr. A. van Keulen

Leden

prof. dr. A. Prosperetti

prof. dr. ir. G.J.M. Krijnen

prof. dr. C.J. Kim

prof. dr. D. Lohse

prof. dr. T.H. van der Meer
Universiteit Twente

Universiteit Twente

Universiteit Twente

Technische Universiteit Delft

Universiteit Twente

Universiteit Twente

University of California, Los Angeles

Universiteit Twente

Universiteit Twente

van den Broek, Dennis

Explosive Micro-Bubble Actuator

Ph.D. Thesis, University of Twente, Enschede, The Netherlands

ISBN: 978-90-365-2743-9

Copyright (C) 2008 by D.M. van den Broek, Enschede, The Netherlands 


\section{Explosive Micro-Bubble Actuator}

\section{PROEFSCHRIFT}

ter verkrijging van

de graad van doctor aan de Universiteit Twente op gezag van de rector magnificus prof. dr. W.H.M. Zijm, volgens besluit van het College voor Promoties in het openbaar te verdedigen op vrijdag 31 oktober 2008 om 15:00 uur

door

Dennis Micha van den Broek geboren op 11 augustus 1979 te Vlaardingen 
Dit proefschrift is goedgekeurd door de promotor:

prof. dr. M.C. Elwenspoek 
aan mijn vader 


\section{Contents}

1 Actuators and Bubbles 1

1.1 Introduction . . . . . . . . . . . . . . . . . . . . . . . . 2

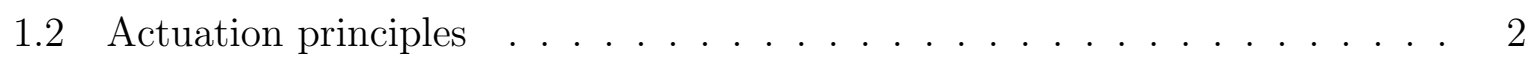

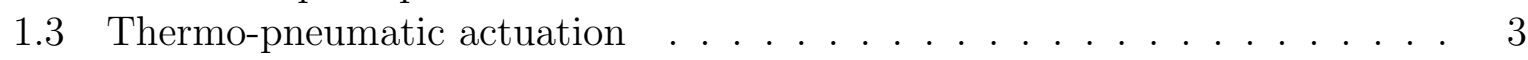

1.3.1 Thermal expansion . . . . . . . . . . . . . . . 3

1.3.2 Phase-change . . . . . . . . . . . . . . . . 3

1.3 .3 Actuator design . . . . . . . . . . . . . . . . . . . . . . . . . . . . . .

1.4 Bubble actuators . . . . . . . . . . . . . . . 5

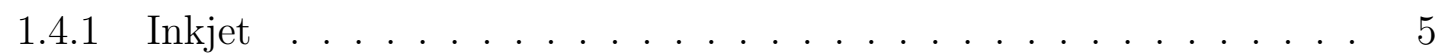

1.4 .2 Bubble pump ......................... 5

1.4.3 Mechanical displacement . . . . . . . . . . . . . . . . . . 6

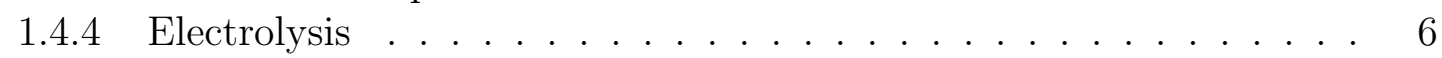

1.5 Bubble nucleation and bubble dynamics . . . . . . . . . . . . . 6

1.6 Motivation of the work . . . . . . . . . . . . . . 8

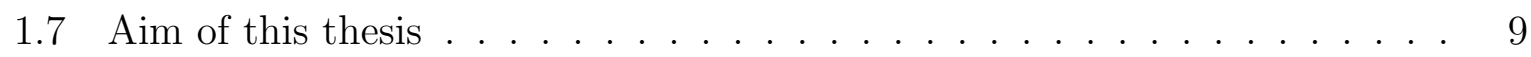

1.8 Outline of the thesis $\ldots \ldots \ldots \ldots \ldots$

2 Theory 11

2.1 Introduction . . . . . . . . . . . . . . . . . . . . 12

2.2 Heat transfer . . . . . . . . . . . . . . . . . . . . . . . 12

2.2.1 The 1-D conduction model . . . . . . . . . . . . . . . 13

2.2.2 The 2-D conduction model . . . . . . . . . . . . . . . 18

2.2.3 Repetition frequency . . . . . . . . . . . . . . . 25

2.2.4 Summary and conclusions on the heat transfer . . . . . . . . . . . 28

2.3 Bubble Generation . . . . . . . . . . . . . . . . . . . . . . 29

2.3.1 The phase transition . . . . . . . . . . . . . . . 30

2.3.2 Kinetic limit of superheat . . . . . . . . . . . . . . . 31

2.3.3 Nucleation probability . . . . . . . . . . . . . . . . 33

2.3.4 Spontaneous nucleation . . . . . . . . . . . . . . 35

2.4 Bubble dynamics . . . . . . . . . . . . . . . . . . . . . . . 36

2.4.1 Explosive evaporation . . . . . . . . . . . . . . . . 37

2.4 .2 Bubble collapse . . . . . . . . . . . . . . . . . . 39

2.4.3 The intermediate stage . . . . . . . . . . . . . . . . . . 39

2.4.4 Summary and conclusions on the bubble dynamics . . . . . . . . 39

2.5 Membrane deflection theory . . . . . . . . . . . . . . . . 40 
2.5.1 Static deflection . . . . . . . . . . . . . . . 41

2.5.2 Membrane vibrations . . . . . . . . . . . . . . . . . . 41

2.5.3 Summary and conclusions on the membrane response . . . . . . . . 46

3 Design and Fabrication $\quad 49$

3.1 Introduction . . . . . . . . . . . . . . . . . 50

3.2 Design and fabrication of the heaters . . . . . . . . . . . . 50

3.2 .1 Design . . . . . . . . . . . . . . . . . 52

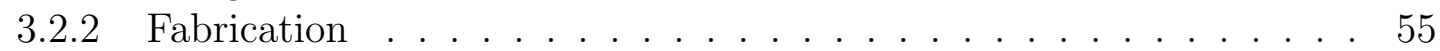

3.3 Fabrication by $\mathrm{KOH}$ etching . . . . . . . . . . . . . 56

3.3.1 Mask design . . . . . . . . . . . . . . . . . . . 57

3.4 Fabrication with Reactive ion etching . . . . . . . . . . . . 60

3.5 The completed device . . . . . . . . . . . . . . . . . 61

3.6 Experimental setup . . . . . . . . . . . . . . . . . . . . 62

4 Bubble dynamics $\quad 65$

4.1 Introduction . . . . . . . . . . . . . . . . . 66

4.2 Stroboscopic imaging . . . . . . . . . . . . . . . . . 66

4.3 Resistance Thermometry . . . . . . . . . . . . . . . . . 67

4.3.1 Calibration ....................... 68

4.4 Bubble nucleation . . . . . . . . . . . . . . . . . . . . . . . . . 69

4.4 .1 Imaging . . . . . . . . . . . . . . . . . . . . 69

4.4 .2 Average heater temperature . . . . . . . . . . . . . . 69

4.4.3 Influence of applied power on the bubble nucleation . . . . . . . . . 70

4.4 .4 Reproducibility . . . . . . . . . . . . . . . 74

4.5 Different heater geometries . . . . . . . . . . . . . . 76

4.5.1 Early nucleation . . . . . . . . . . . . . . . . . . . . . . . . . . . . . 78

4.5.2 Current density simulation . . . . . . . . . . . . . . . . . . . 79

4.5.3 Effects of early nucleation . . . . . . . . . . . . . . . 80

4.6 Bubble growth and collapse . . . . . . . . . . . . . . . . 81

4.6.1 The growth stage . . . . . . . . . . . . . . . . . . 82

4.6.2 The intermediate stage . . . . . . . . . . . . . . . . 84

4.6 .3 The final stage . . . . . . . . . . . . . . . . . 87

4.6.4 The influence of the cavity pressure . . . . . . . . . . . . . . . 90

4.7 Repetition frequency . . . . . . . . . . . . . . . . . . 93

4.7.1 Effects on the temperature . . . . . . . . . . . . . 94

4.7.2 Effect on the bubble nucleation . . . . . . . . . . . . . 96

4.7.3 Effect on the bubble growth and collapse . . . . . . . . . . . . . . 100

4.8 Summary and conclusions . . . . . . . . . . . . . . . . . . 102

5 Response of the membrane $\quad 105$

5.1 Introduction . . . . . . . . . . . . . . . . 106

5.2 Static deflection . . . . . . . . . . . . . . . 106

5.2.1 Different membrane sizes . . . . . . . . . . . . . . . . . . . 108

5.3 Dynamic deflection . . . . . . . . . . . . . . . . . . . 110 
5.3.1 Effect of the membrane geometry . . . . . . . . . . . . . . . . 112

5.3.2 Effect of the membrane size . . . . . . . . . . . . . . . . 115

5.3.3 Effect of the applied heating power . . . . . . . . . . . . 116

5.3.4 Effect of the cavity pressure . . . . . . . . . . . . . . . 117

5.4 Initial dynamic deflection . . . . . . . . . . . . . . . . . . . 119

5.4.1 Nucleation and the maximum deflection . . . . . . . . . . . . 120

5.4.2 Effect of the heater size and the membrane size . . . . . . . . . . 123

5.4.3 Effect of the pressure inside the cavity . . . . . . . . . . . . . 129

5.5 Ideal response . . . . . . . . . . . . . . . . . . . . . . . . 132

5.6 Effect of the repetition frequency . . . . . . . . . . . . . 133

5.6.1 Deflection control . . . . . . . . . . . . . . . 135

5.6.2 Influence of the cavity pressure at high repetition frequencies . . . . 136

5.7 Summary and conclusions . . . . . . . . . . . . . . . . 138

6 Conclusion and Outlook $\quad 141$

6.1 Overall conclusion . . . . . . . . . . . . . . . . . . . . . . . . . 142

6.2 Design improvements . . . . . . . . . . . . . . . . . . 145

6.2.1 Heaters and substrate . . . . . . . . . . . . . . . . . . 145

6.2.2 Optimal properties of the liquid . . . . . . . . . . . . . . . . 149

6.2 .3 Membrane . . . . . . . . . . . . . . . . . . . . . 151

6.3 Applications . . . . . . . . . . . . . . . . . . 151

$\begin{array}{ll}\text { References } & 155\end{array}$

$\begin{array}{ll}\text { Summary } & 163\end{array}$

$\begin{array}{ll}\text { Samenvatting } & 165\end{array}$

$\begin{array}{ll}\text { Dankwoord } & 167\end{array}$ 


\section{Chapter 1}

\section{Actuators and Bubbles}

A large variety of actuators can be found in literature. This chapter provides a brief overview of different actuator principles with a focus on thermo-pnuematic actuation and actuators, which utilize bubbles to provide the actuation force. The second part of this chapter will give a summary of the research on bubble nucleation and bubble dynamics under high heat-flux conditions. At the end of this chapter, an outline of this thesis is given. 


\subsection{Introduction}

The last decades there has been a trend to miniaturize systems of virtually any kind. This trend has many reasons: small systems are potentially cheaper to produce, they can have properties large systems have not, they may facilitate use, and they can be more energy efficient. This miniaturization has led to systems of a few $\mathrm{mm}^{3}$ and smaller. These microsystems have typical details in the micrometer range and Micro Electrical Mechanical Systems technology is used to fabricate these systems. In general microsystems comprise of sensors, components that deliver some information, intelligence to process information and decide over some particular action, and an actuator that performs the action. Several types of forces can be used to perform an action. This resulted in a large number of different microactuator types and an even larger number of actuator designs. The microbubble actuator uses the principle of thermo-pneumatic actuation. The introduction of a large amount of heat causes explosive evaporation and creates a bubble in a liquid. The pressure impulse generated by the formation of a bubble will cause a membrane deflection. Although the force generated by bubbles is used in several successful actuator designs, bubble generation and bubble dynamics are not completely understood and still topics of research.

\section{$1.2 \quad$ Actuation principles}

There are many ways to generate a force and therefore many different actuator principles. In general MEMS actuators can placed in four different families and each family can be divided in several classes. The four families are:

- Electrostatic

- Magnetic

- Thermal

- Piezoelectric

The type of actuator used in a microsystem depends on the required performance of the actuator. Here one can think of the generated force, the displacement and repetition frequency. This performance is different for each family and each family has its limitations. Table 1.1 shows the performance and the limitations of the different families.

\begin{tabular}{|c|c|c|c|c|}
\hline & Force & Stroke & Frequency & Limitation \\
\hline Electrostatic & - & $+/-$ & + & Weak \\
Piezoelectric & $+/-$ & $+/-$ & + & Material \\
Thermal & + & + & - & Low frequency \\
Magnetic & - & - & $+/-$ & Weak \\
\hline
\end{tabular}

Table 1.1: Preformance and limitations of the different actuator families 
Electrostatic and magnetic actuators are relatively weak, especially if the dimensions of the actuator have to be small. Piezoelectric materials are hard to incorporate into the present MEMS fabrication techniques and this limits the use of the piezoelectric effect in micro-actuators. Thermal actuators can generate relatively large force and a large stroke, but the repetition frequency is limited to a couple of hundred Hertz due to the relatively slow process of heat diffusion.

\subsection{Thermo-pneumatic actuation}

The micro-bubble actuator can be classified as a thermo-pneumatic actuator. The thermopneumatic actuators belongs to the thermal actuator family and uses the pressure generated by adding heat to the system to produce work.

\subsubsection{Thermal expansion}

Most materials expand upon heating. If this expansion occurs in an area with a fixed volume a pressure buildup will occur. This excessive pressure can be used to perform mechanical work. The simplest systems consist of a small cavity filled with a material, in most cases a fluid, that expands upon heating. This can be either a liquid, like water, isopropanol or special designated fluids like the Performance Fluids fabricated by $3 \mathrm{M}[1,2]$ or a gas, which in most cases is normal air $[3,2,4,5,6,7,8,9,10,11,12]$. On one side the cavity is sealed off by a membrane. A heater is placed inside the cavity to supply the heat needed for expansion. The expanding fluid will lead to a pressure increase inside the cavity and a membrane deflection. The pressure inside the cavity and thus the membrane deflection is directly related to the temperature of the working liquid.

\subsubsection{Phase-change}

Heating a material can also result in a phase change. In general a phase change also corresponds to a significant change in material properties, such as the density. For example the transfer from the liquid phase to the vapour phase of a fluid generally leads to a much larger volume expansion than a normal thermal expansion. Therefore it can generate considerable forces in small volumes. The working material can be a liquid, for example water, pentane, ethanol,a refrigerant like freon-11 or the earlier mentioned Preformance Fluids $[13,14,15,16]$. The phase change from the solid to the liquid phase will also result in volume expansion for most materials. In this case Paraffin is often used as the working material $[17,18]$.

\subsubsection{Actuator design}

Thermo-pneumatic actuators are used as pumps or valves. In both cases a deflecting membrane will be used to provide the intended action. The main difference between the two applications is that a valve requires a continuous deflection to keep a certain state (for example open or closed). In most cases the valve is open in the initial state and a continuous heating power has to be applied to close the valve, these valves are thus 
normally open. An example of a normally closed valve is given by Henning et al. [19]. In general the design of these valves is much more complicated than for a normally open valve. Both types of valves require a continuous power to keep the valve out of its normal position. The bistable valve fabricated by Goll et all. [3] uses a prebuckled membrane to create two stable states. This type of microvalve thus only needs power during switching.

In a pump the deflection of the membrane results in the displacement of certain volume of fluid. To transport the fluid through the system a flow in a certain direction must be generated. There are several ways to achieve such a flow. Valves can regulate the direction of the flow. Most often passive valves $[8,14,12]$ are used, because active valves require actuators to control the valves.

The number of moving components in the pump can be minimized by using nozzle/diffuser structure. A vibrating membrane combined with the different flow characteristics of the nozzle/diffuser will lead to a fluid flow $[4,5,18]$. A liquid flow can also be generated when a number of actuators are placed in series in a channel to form a peristaltic pump $[7,20]$. If the membranes of the individual actuators are deflected following the correct sequence a fluid flow will be generated in a certain direction.

As discussed in 1.3.1 the simplest thermo-pneumatic actuators consist of a cavity filled with a working material sealed on one side by a flexible membrane. MEMS fabrication techniques are used to fabricated the cavity, the membrane and other structures. Therefore most of the actuators are made from silicon, glass or Polydimethylsiloxaan (PDMS). The membrane has to be strong, but also flexible to get a considerable deflection. Several materials are used to form a membrane. Polymers like silicon rubber[14, 1, 2], parylene[2], polymide [21, 3], PDMS [6, 7, 10, 18, 20], Kapton [9, 8], Mylar [8] are used because of their strength and flexibility, but the silicon itself is also used as a membrane material $[4,13,5,19,15,12]$. In order to reduce the residual stress and generate a larger deflection a corrugated membrane can be used instead of a flat membrane [5, 4, 13]. Identical to the membrane the heaters, which are needed to heat the working material, are also made from different materials. In most cases metals like gold, copper or platinum are used, but heaters were also made form highly doped silicon. This enabled the fabrication of an actuator completely based on silicon technology[13, 5].

The transfer of heat between the different parts of the actuator is very important for its performance. A large part of the heat generated by the heater will be lost in the substrate if the heater is placed directly on the substrate. To reduce the heat loss to the substrate heaters are placed on a membranes $[2,13,7]$ or suspended in the cavity containing the working material[15, 10]. The maximum operating frequency depends not only on the time it takes the actuator to heat the working liquid and generate a membrane deflection, but also on the time it requires to return to its initial state. This last step takes much more time $[15,9]$ and often is the limiting step for the maximum frequency. For all the thermo-pneumatic actuators mentioned in this section the maximum operating frequency is only several Herz. The highest frequency of $100 \mathrm{hz}$ was reported for a peristaltic pump fabricated by Jeong et al. [20]. The maximum flow for this pump however was generated at a repetition frequency of $2 \mathrm{~Hz}$. The maximum deflection generated by the different actuators depends on the design, but are generally several microns. The largest deflection found in literature for a membrane actuator made with MEMS technology was $375 \mu \mathrm{m}$ [9]. 


\subsection{Bubble actuators}

The explosive micro-bubble actuator will use the pressure impulse generated by bubbles created in a working liquid to deflect a membrane. This is the first time that bubbles are used in a thermo-pneumatic actuator to deflect a membrane. The mechanical work generated by micro-bubbles, however, is already successfully used in a number of microsystems.

\subsubsection{Inkjet}

One of the most successful applications of bubbles in a micro-system is the thermal bubble jet printer. Vapour bubbles generated by a rapid heating of ink in nozzles act as pressure generators $[22,23,24]$. Small droplets are ejected from the nozzle with a high precision and with frequencies up to $35 \mathrm{kHz}[25]$. The size and the speed of the droplets and the maximum frequency are highly dependent on the dynamics of the generated vapour bubbles. Kang et al. [26] used an array of microheaters to control the generated bubble volume and thus volume of the ejected droplet. In most bubble jet systems the ejected droplets and the vaporized liquid are of the same material. If however a high viscosity liquid is used as a fluid plug, a droplet of this liquid is fired from a nozzle after a bubble is created in a low-boiling temperature liquid propellant [27].

\subsubsection{Bubble pump}

A growing or collapsing bubble will exert a force on the surrounding liquid and in an open system this will result in a liquid flow. The movement of the bubble interface can be used to pump a fluid. In order to create a pumping effect there has to be some asymmetry. This asymmetry can be achieved by using a nozzle/diffuser structure [28, 29]. The bubble is created and controlled in a pumping chamber by a heater. One side of the chamber is connected to a nozzle and another side is connected to the diffuser. As the bubble expands, the flow at the diffuser is larger than at the nozzle and when the bubble collapses the flow at the diffuser is smaller than at the nozzle. Therefore a net flow is generated from the nozzle to the diffuser during each growth and collapse cycle.

Another way to create an asymmetry is achieved by creating a bubble inside a channel. The heater, at which the bubble is generated, is located closer to one end of the channel than the other [30]. The asymmetric placement of the heater and thus of the bubble location results in a net flow, if the collapse and growth are periodically cycled. Placing more heaters in the channel made it possible to apply a temperature gradient across the channel $[31,32]$. The generation and movement of bubbles can be manipulated by changing this gradient. The variation in vapour pressure and surface tension along the channel can also be used to create a pumping effect.

A bubble formed in a small channel can not only pump a liquid, but can also be used to block the channel and prevent liquid flow. The bubble can thus be used as a valve. Van der Wijngaart et al. [33] used gas bubbles created in a PDMS channel structure as microvalves. 


\subsubsection{Mechanical displacement}

The explosive micro-bubble actuator is the first actuator that uses the forces generated by thermal bubbles to displace a membrane. Nevertheless bubbles were already used to displace other mechanical structures. The deflection of a microcantilever generated by a growing vapour bubble was studied by Lin et al. [34] and by Lee et al. [35]. A floating gate structure was fabricated by Papavasiliou et al. [36] and used as a valve.

\subsubsection{Electrolysis}

Bubbles can also be generated by electrolysis. By passing a current through aqueous solution gas bubbles are created. Identical to thermally created bubbles these bubbles can perform work. A comparative study of electrolysis and boiling for bubble-driven microactuations was done by Meng et al.[37]. Examples of actuators, which use electrolysis to generate bubbles, are an active valve fabricated by Neagu et al. [38], the floating gate described in the previous section [39] and a T-switch created by Ho et al. [40].

\subsection{Bubble nucleation and bubble dynamics}

The fact that liquid can be superheated and bubbles can grow in a super heated liquid is known for a long time. In 1953 Plesset and Zwick [41] described the growth of vapour bubbles in a superheated liquid and a detailed description of bubble nucleation in liquids is given by Blander and Katz [42]. The latter gives a review of theoretical and experimental aspects of homogeneous and heterogeneous bubble nucleation. An extensive research on bubble nucleation and especially on explosive boiling was done by Skripov [43]. His book titled Metastable Liquids describes the theory behind explosive boiling and presents the results of his research. Another work on bubble nucleation and growth, where the different types and stages of boiling are discussed, was written by van Stralen [44].

In spite of the extensive research on bubble nucleation and growth in the past there are still a lot of questions on this subject and numbers of papers were written relatively recently on the subject $[45,46,47,48,49,50,51]$. In each paper a small part of the process is investigated and described, extending the theory somewhat further. Experiments were done mostly with thin metal wires $[42,43,52,53]$ and in a few cases on relatively large planar heaters [54]. Main focus in these experiments was the maximum attainable superheat and heat transfer characteristics of the wire in the working liquid. With the advancing MEMS technology the fabrication of heaters with sizes down to microns or even sub-microns became possible. The decrease in heater size resulted in an increase heat-flux and explosive boiling in small structures became possible. This resulted in the development of the first bubble jet printers. In these inkjet printers small droplets of ink are ejected from a nozzle due to the high pressure in vapour bubbles, generated by rapid heating of the ink.

Asai et al. were the first to produce a model, which described the bubble growth and liquid flow in a bubblejet [22]. The model was divided in three parts. In the first the initial heating of the ink was calculated by using a 1-D conduction model and the total energy production. The second part dealt with the bubble growth and collapse together with the 
droplet formation utilizing the Clausius-Clapeyron equation and energy conservation. The last part of the model described the refilling of the nozzle by surface tension. In the same paper numerical calculations on the bubble jet system, which show that the nucleation temperature is estimated to be $270^{\circ} \mathrm{C}$ and initial pressure $4,5 \mathrm{MPa}$, are presented. These results are compared with measurements done on a real bubble jet for three different types of ink. This paper and another article [55], where the classical nucleation theory is applied to the initial stage of bubble formation, are combined in [56]. The model for the bubble dynamics is supported by top and side view images of bubbles created in the liquid for various heat-fluxes. More insight was gained by solving the three dimensional Navier-Stokes equation for the bubble jet printer [57].

The success of the bubble jet printer has lead to a fast increase in the number of studies on the nucleation and dynamics of bubbles generated under high heat-flux conditions. Iida et al. [58] investigated the bubble generation in ethyl alcohol on a $100 \times 250 \mu \mathrm{m}^{2}$ heater for heating rates over $10^{7} \mathrm{~K} / \mathrm{s}$ and saw a "caviarwise bubble generation". The same heater was used to investigate the bubble generation in toluene and water [59]. From images of the heater directly after the moment of nucleation a bubble number density is estimated and the results were compared with the homogeneous and heterogeneous nucleation theory. Avedisian et al. [60] used the heater of commercial inkjet to investigate the bubble nucleation in water. The heater resistance was calibrated before measurements. With this calibration curve the average temperature could be estimate from the resistance during heating pulses. Lin et al. [61] were the first to use a heater made of polysilicon and because the development of MEMS technology the effect of a heater with a irregular shape could be measured. Further investigations on the growth and the collapse of bubbles created due to pulse heating by a micrometer sized heater are described in following papers $[62,63,64,65,66,23,67,50]$.

Bubbles normally are assumed to be spherical, but if one applies a high heat-flux the generated bubbles cover the heater with a film of vapour. The height of this vapour film is hard to measure. The light intensity at the edges of the bubble depends on the curvature of the bubble interface. Yin et al. [68] used this effect to get an idea about the bubble shape for different heat fluxes. Another method to get an insight in the bubble shape is to set the focal plane on different heights, thus getting a complete image of the bubble shape [69]. The most straight forward method is by combining the top and side-view images. Side-view images can be taken by using two microscopes in a perpendicular arrangement or by tilting the sample under a $90^{\circ}$ angle. This is not as simple as it sounds because in most cases the focal distance of the microscope limits the freedom to move the sample around. However Hong et al. [70] managed to make top and side view images of bubble growth and compared the results with the results from 3-D numerical simulation presented in the same paper. Also the articles of Asai [56], Okuyma et al. [71] and Varlamov et al. [72] contain side-view images. It can be seen that especially for high heating rates the height of the initial bubble is relatively small and thus the bubble cannot be assumed spherical. At repetition frequencies of the heat pulses greater than $20 \mathrm{~Hz}$ Varlamov et al. [72] observed the formation of even more complicated bubble structures, which where the result of the interaction between the bubble created on the heater surface and a gas bubble situated near the heater surface.

In order to use a bubble to drive an actuator it necessary to find a way to measure 
the force generate by the expanding and collapsing bubble. This can be done by measuring the acoustic pressure waves emitted by the bubble $[73,74]$. It is also important to know the temperature in the substrate and liquid for the heater working at high frequencies, especially when the working liquid contains degradable molecules, for example DNA molecules $[75,76,77]$. For these cases one can calculate a allowable repetition frequency for the device [78], this is the maximum frequency for which a specific part of the sample stays below certain temperature if the device is used in a repetition mode.

\subsection{Motivation of the work}

Microactuators are key components in numerous microsystems. Several principles can be used to generate a force. All the principles have their specific drawbacks and advantages. In general electrostatic actuators are fast but weak, thermal actuators are strong but slow and piezoelectric actuators require high actuation voltages and the materials are difficult to process with standard micro-fabrication technologies.

In many applications strong and fast microactuators are required. Examples are positioning of optical components such as mirrors or lenses, instruments in micromanipulation, actuators for pumps and valves, loudspeakers and ultrasoundgenerators. Therefore there is much effort put into the search for alternative actuator principles.

In this project an actuator, which is potentially fast and strong will be designed and investigated. It employs the explosive like generation of vapour bubbles in a liquid when a small electric resistor is heated quickly. Microtechnology offers the possibility to fabricate structures with small feature size. Diffusion processes generally limit the kinetics of thermal actuators. The micro-bubble actuator has the dimension in the micrometer range and will be heated by microsecond pulses. The diffusion will take place over short distances which makes the actuator potentially fast. Combined with the large pressure that can be generated in the thermal domain, the actuator should also be strong.

Typically actuators using the principle of a thermodynamic phase change are slow because the diffusion processes rule the dynamics. However, if heat is generated fast enough the liquid surrounding the heating element cannot diffuse away and a small volume of superheated liquid is the result. The same principle is used in the thermal inkjet printers. The basic difference is that here the working liquid is contained in a closed system, which is meant to displace a membrane, while in ink jet printers the liquid itself is displaced and continuously renewed.

The bubble formation and dynamics are very complex and still a topic of research. Despite the commercial success of the inkjet printer, the number of studies on the bubble generation, growth and collapse is limited. A complete model, which treads all aspects of bubble generation and the resulting pressure- and temperature field is lacking. Investigation of the bubbles under these high heat-flux conditions will increase the knowledge of these processes. This information can be used to create a complete model and will help to improve the design of the actuator. 


\subsection{Aim of this thesis}

The aim of the project was to design an actuator, which uses explosive evaporation to create a bubble inside a closed cavity. The pressure generated during the bubble growth will be utilized to deflect a membrane (figure 1.1). The explosive micro-bubble actuator will be fabricated using standard MEMS technology.

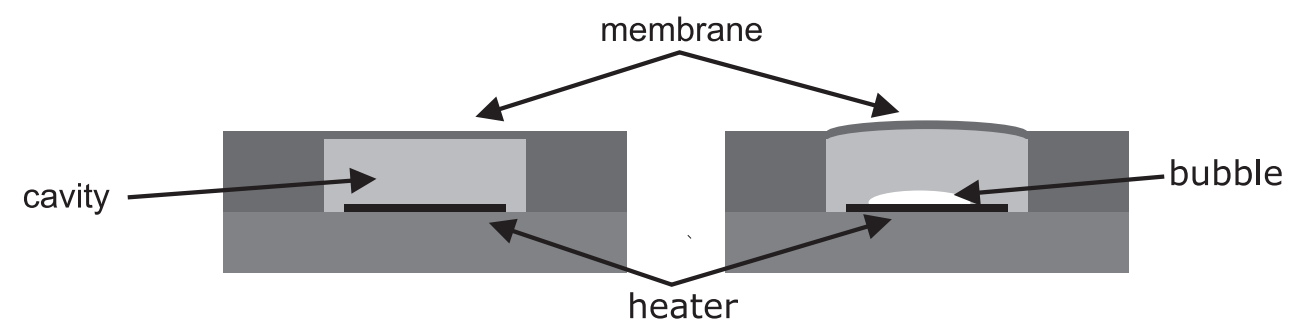

Figure 1.1: Simplified images of the bubble actuator showing the working principle. The left image shows the actuator before heating and the right image shows the actuator with a bubble inside the cavity formed by a short heating pulse from a heater at the bottom of the cavity.

In this thesis the possibilities and the limitations of the explosive micro bubble actuator will be investigated. In particular the maximum membrane deflection and the maximum operation frequency will be examined. The influence of different operating and design parameters, such a heating power and heater geometry, on the performance is studied. This information can be used to find the best operating conditions and design rules for future devices.

The performance of the actuator will be highly dependent on the generation and dynamics of the created bubbles. Heating conditions, such as heating power and pulse length, will have a considerable effect on the generation of bubbles by explosive evaporation. Also in the further development of the bubbles these parameters will be important. A study of the influence of these variables and other parameters will help to increase the insight in the bubble dynamics and provide the necessary experimental data to verify the validity of newly developed models.

An actuator, which generates deflection of several micrometers at repetition frequencies of several $\mathrm{kHz}$, can be used in several applications. The specific requirements of the actuator performance will be different for each application. The results from this research and the developed design rules can be used to create a device fulfilling the requirements for the specific application and find the conditions for an optimal performance.

\subsection{Outline of the thesis}

The first part of this chapter gave a brief summary of different actuator principles. The working principle and design of several thermopnuematic actuators and bubble actuators was discussed. In the second part of the chapter an overview of research and literature on bubble generation and bubble dynamics under high heat-flux conditions was given. 
In chapter 2 some theoretical aspects of the two main principles of the explosive micro-bubble actuator will be discussed. The first part deals with the heat-transfer in the system, the bubble generation and bubble dynamics. Several simple conduction models are presented and used for numerical simulations to predict the effect of different heating conditions. The bubble generation takes place by high heat-flux heating pulses and this will result in spontaneous nucleation. After bubble generation the bubble grows explosively and finally the vapour bubble will collapse in same manner as a cavitation bubble. The second part of the chapter deals with the response of the membrane. A continuous pressure results in a static deflection of the membrane. The sudden generation and growth of a bubble create a pressure impulse and this will start the dynamic deflection of the membrane. After this initial deflection the membrane returns to its original state in a free-damped vibration.

The design and fabrication of the system, which contains a heater, a cavity filled with liquid and a membrane, will be discussed in chapter 3 . This chapter will also discuss the setup used for the experiments.

The bubble generation and the bubble dynamics were studied by resistance thermometry and stroboscopic imaging and the results are shown in chapter 4 . The effects of the heating parameters, such as pulse length, heating power and repetition frequency, as well as the effect of different heater sizes and geometries were investigated. In contrast to the research on the bubble generation in the ink jet printers and most other studies on bubble generation under high heat-flux conditions, the bubbles in the explosive micro-bubble actuator are generated in a closed system. Therefore influence of the pressure inside the cavity on the bubble generation and dynamics were also examined.

The response of the membrane to the generation of a bubble is treated in chapter 5. The membrane speed and dynamic deflection were measured with a laser-vibrometer. A constant pressure difference across the membrane will result in a static deflection of the membrane. This deflection was measured with a white light interferometer and these measurements can be used to calibrate the membrane. The maximum dynamic deflection and the maximum repetition frequency are two important factors, which determine the overall performance of the actuator. A large number of parameters will effect these factors. The geometry and size of the membrane and the heaters, the pressure inside the cavity and the heating conditions are examples of these parameters.

The last chapter will give an detailed overview of the main conclusions of this thesis. A number of recommendations and improvements to the design will be discussed, as well as some future applications. 


\section{Chapter 2}

\section{Theory}

In this chapter some theoretical aspects of the two main principles of the micro-bubble actuator will be discussed. The first part covers the heat-transfer in the system, the bubble generation and bubble dynamics. The second part of the chapter deals with the response of the membrane. 


\section{$2.1 \quad$ Introduction}

In this chapter the theory behind two main principles of the micro-bubble actuator will be discussed. The first part of the chapter will deal with the transport of heat in the system and the formation of bubbles. The process of bubble generation and collapse can be divided in several steps. First the liquid is heated by joule heating. Short intensive heating pulses are used to superheat the liquid. The theory of heat conduction is discussed and some models are developed to describe the heat-transfer through the system. At the moment of nucleation the liquid will have temperatures close to the critical temperature and explosive evaporation will occur. This will result in spontaneous nucleation. After nucleation, the bubble will grow or shrink depending on the heating conditions. The dynamical behaviour of the bubble is very complex, but with some assumptions it is possible to estimate important parameters like the growth speed, bubble volume and collapse speed. Finally the bubble will collapse and the liquid will cool down to its initial state.

The second part of the chapter will treat the behaviour of the membrane. Two different cases will be discussed; the static case where a constant deflection is caused by a pressure difference across the membrane and the dynamic case where the membrane vibrates due to an excitation.

\section{$2.2 \quad$ Heat transfer}

In the first stage the liquid is heated by a thin film resistor located on a substrate. A potential difference is applied across the heater and a current will flow through the heater. The resistance of the heater results in energy dissipation in the form of heat in the metal layer. As a result the heater will start to heat up. The power $(P)$ generated by joule heating can be calculated using equation(2.1)

$$
P=V I=I^{2} R=\frac{V^{2}}{R}
$$

here $V$ is the applied voltage, $I$ is the resulting current through the heater and $R$ is the resistance of the heater. The generated heat will be distributed through the rest of the system following the laws of thermodynamics. Heat can be transferred by three different mechanisms: conduction, convection and radiation. Before the bubble is generated the liquid is at rest and the heat-transfer due to convection can be neglected in the first stage. The contribution due to radiation is still relatively small at the temperatures reached during the heating pulse and will therefore be neglected in this case, this only leaves heat transfer due to conduction. In this case the heat transfer is governed by two fundamental equations [79]. The first describes the heat transfer by conduction:

$$
\frac{\partial T}{\partial t}=D \nabla^{2} T
$$

here $T$ is the temperature and $D$ is the thermal diffusivity. The thermal diffusivity $D$ is related to the the thermal conductivity $k$ by:

$$
D=\frac{k}{\rho c_{P}}
$$


where $\rho$ is the density and $c_{p}$ is the isobaric heat capacity. The second equation defines the temperature gradient for a certain heat-flux $(q)$ :

$$
k \nabla T=q
$$

\subsubsection{The 1-D conduction model}

A simple model was constructed to get an estimate of the surface temperature of the heater and the temperature of the liquid surrounding the heater during the first step. The heating pulse will be short and the heat penetrates only a small distance into the liquid and substrate. This distance will be much smaller than the length and width of the heater. Therefore the conduction can be treated as one-dimensional. The thermal diffusion length $l_{d}$ for the one-dimensional case is given by

$$
l_{d}=\sqrt{\pi D t}
$$

where $t$ is the pulse length. For pulses of $10 \mu$ s and a thermal diffusivity of pyrex, which will be used as a substrate, the thermal diffusion length is about $4 \mu \mathrm{m}$. At the end of the heating pulse only a layer of a few microns will have an elevated temperature. Furthermore the thickness of the heater and its high thermal conductivity makes it possible to model it as an infinitely thin wire. The heat-flux produced by this wire is assumed constant and uniform. The working liquid is situated on one side of the wire and a solid, in this case the pyrex substrate, is located on the other side. A simplified image of the model is shown in figure 2.1 .

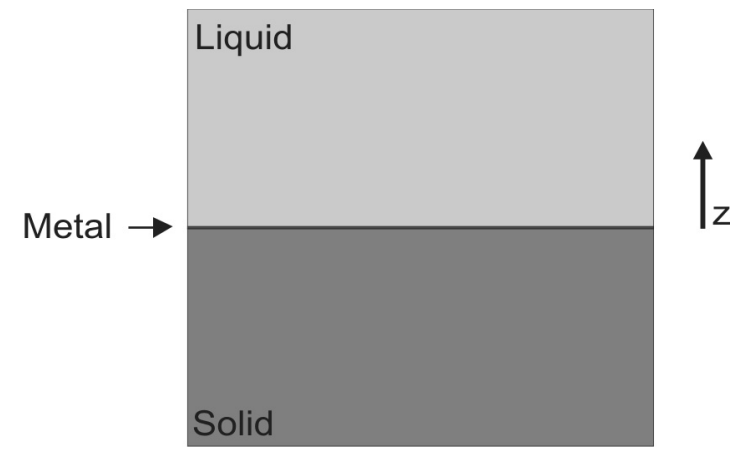

Figure 2.1: A schematic drawing of the 1-D conduction model

This model can be solved analytically using the following boundary conditions:

$$
\begin{aligned}
T & =T_{0} \text { at } t=0 \text { and } z=\infty \\
-\left.k_{l} \frac{\partial T}{\partial z}\right|_{z=0^{+}}+\left.k_{s} \frac{\partial T}{\partial z}\right|_{z=0^{-}} & =q
\end{aligned}
$$

where the subscribts $l$ and $s$ represent the liquid and solid respectively. The analytical solution of the 1-D model in the positive $\mathrm{z}$ direction and thus for the liquid is:

$$
T=\frac{q}{\frac{k_{s}}{\sqrt{D_{s}}}+\frac{k_{l}}{\sqrt{D_{l}}}}\left[2 \sqrt{\frac{t}{\pi}} e^{-\frac{z^{2}}{4 D_{l} t}}-z \frac{\operatorname{erfc}\left(\frac{\mathrm{z}}{\sqrt{4 \mathrm{D}_{1} \mathrm{t}}}\right)}{\sqrt{D_{l}}}\right]+T_{0}
$$


For $z=0$ the heater temperature is obtained:

$$
T=\frac{2 q \sqrt{\frac{t}{\pi}}}{\frac{k_{s}}{\sqrt{D_{s}}}+\frac{k_{l}}{\sqrt{D_{l}}}}+T_{0}
$$

here $D_{l}$ and $D_{s}$ are the heat-diffusivity of the liquid and the solid. From this equation it can be seen that the surface temperature of the heater increases with the square root of time and depends linearly on the heat-flux supplied by the heater.

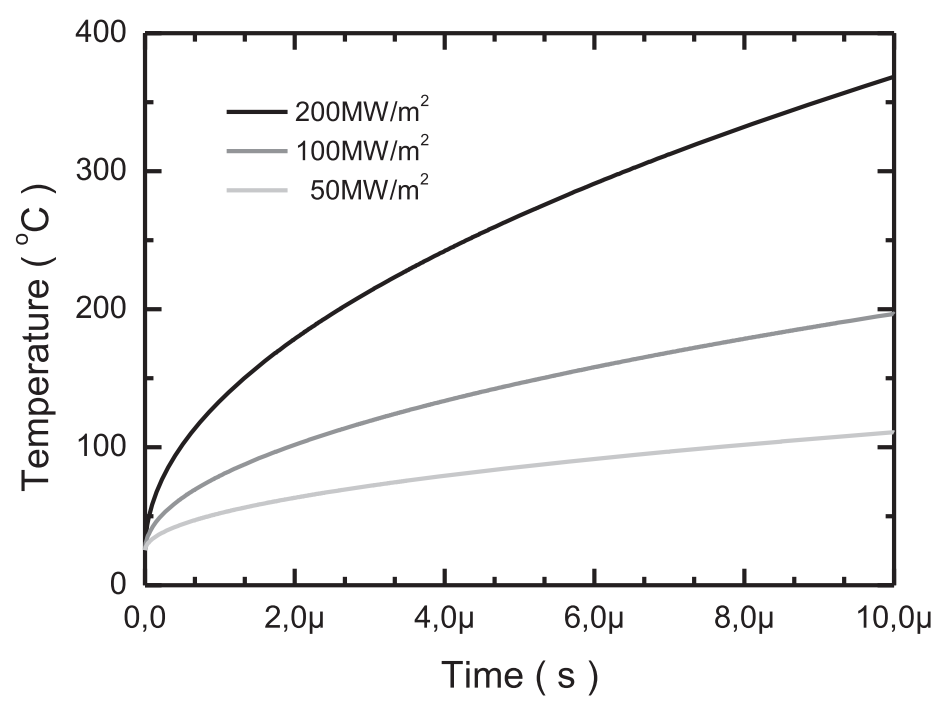

Figure 2.2: Temperature of the heater $(z=0)$ during a heating pulse for 3 different values of the applied heat-fluxes.

Figure 2.2 shows the calculated heater surface temperature in time for three different applied heat-fluxes. In this calculation we used the material properties of ethanol for the liquid part and of pyrex for the solid part. The combination of the two terms in the denominator of equation (2.8) determines how much energy is needed to reach a certain temperature. The amount of energy actually transferred into the liquid depends on the distribution between the two terms. Both terms are composed of the ratio between thermal conductivity and the square root of the thermal diffusivity. The relation between the thermal properties of the liquid and the solid substrate thus regulates how much energy is lost in the substrate. The combination of the two determines the total energy necessary to get a particular temperature difference.

Figure 2.3 shows the heater temperature versus time for three liquids commonly used in these types of experiments. The thermal properties of the liquids are listed in table 2.1. This table also contains the values for pyrex and silicon, both are materials regularly used as substrates. For water the heater temperature increases much more slowly than for ethanol and FC-72, because the combined terms of the thermal properties of water and a pyrex substrate have the highest value. The produced energy however is used more efficiently, more than $50 \%$ of the produced energy goes into the water and the rest is lost in the substrate. For ethanol and FC-72 respectivily about $70 \%$ and $80 \%$ of the produced 


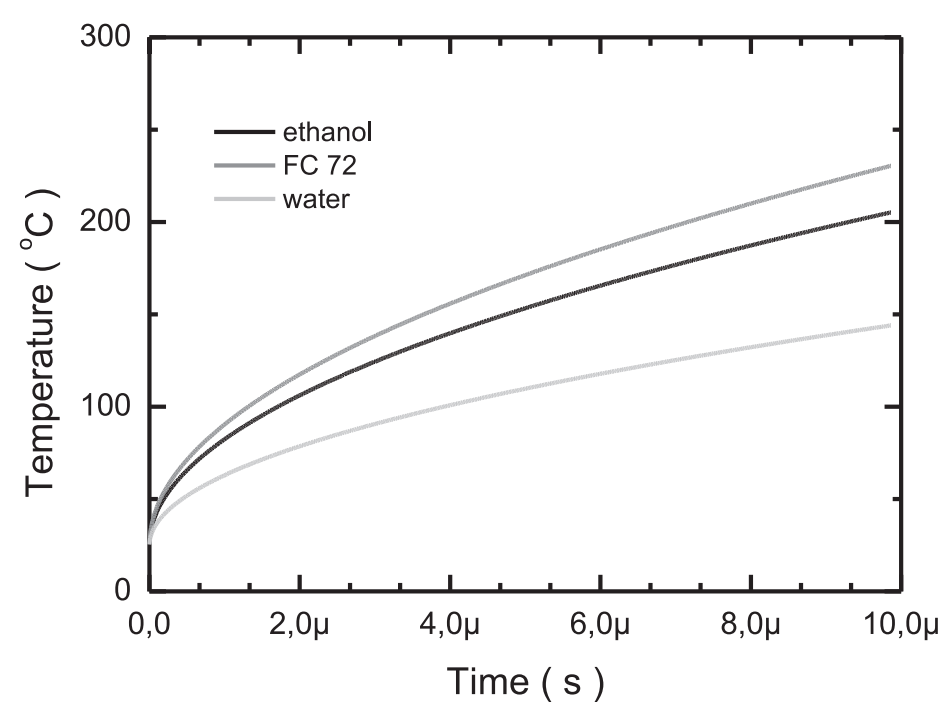

Figure 2.3: Temperature of the heater $(z=0)$ during a heating pulse for ethanol, FC-72 and water.

\begin{tabular}{|l|c|c|c|c|c|}
\hline & ethanol & water & FC-72 & pyrex & silicon \\
\hline$k(\mathrm{~W} / \mathrm{mK})$ & 0.169 & 0.569 & 0.057 & 1.1 & 163 \\
$\rho\left(\mathrm{kg} / \mathrm{m}^{3}\right)$ & 789 & 997 & 1680 & 2230 & 2230 \\
$C_{p}(\mathrm{~J} / \mathrm{kgK})$ & 2400 & 4185 & 1100 & 800 & 700 \\
$D\left(\mathrm{~m}^{2} / \mathrm{s}\right)$ & $8.9 \times 10^{-8}$ & $1.3 \times 10^{-7}$ & $3.0 \times 10^{-8}$ & $6.1 \times 10^{-7}$ & $1.0 \times 10^{-4}$ \\
$T_{b}\left({ }^{\circ} \mathrm{C}\right)$ & 78 & 100 & 56 & - & - \\
\hline
\end{tabular}

Table 2.1: Thermal properties of different liquids and solids.

energy go to the substrate. For silicon, which has a much higher thermal conductivity, more than $90 \%$ of the heat will go to the substrate for all the liquids.

Figure 2.4 shows the temperature distribution in the liquid after $10 \mu$ s of heating at a $100 \mathrm{MW} / \mathrm{m}^{2}$ heat-flux. The distribution is determined by the diffusivity of the liquid. A low diffusivity means a slow diffusion of heat into the liquid. The temperature gradient will be large and a relatively small volume will thus be significantly heated. The maximum temperature of the FC-72, which is reached at the heater, is much higher than the temperature of water after the $10 \mu$ s pulse. However, at a distance of $500 \mathrm{~nm}$ from the heater both liquids will have the same temperature at the end of a $10 \mu$ s heating pulse. About $2 \mu \mathrm{m}$ from the heater almost no change of temperature will take place if FC-72 is used, but for water the distance is doubled and lies $4 \mu \mathrm{m}$ from the heater. This was already predicted by equation (2.5) were a larger diffusivity leads to a larger diffusion length.

The temperature distribution will have a large influence on the moment of bubble nucleation and the size of the initial bubble. The bubble nucleation itself will be discussed further in section 2.3, but for now it is just assumed that the nucleation takes place at the end of the heating pulse. In reality bubble nucleation can also take place before or after the end of the heating pulse depending on the heating conditions. Only the liquid with 


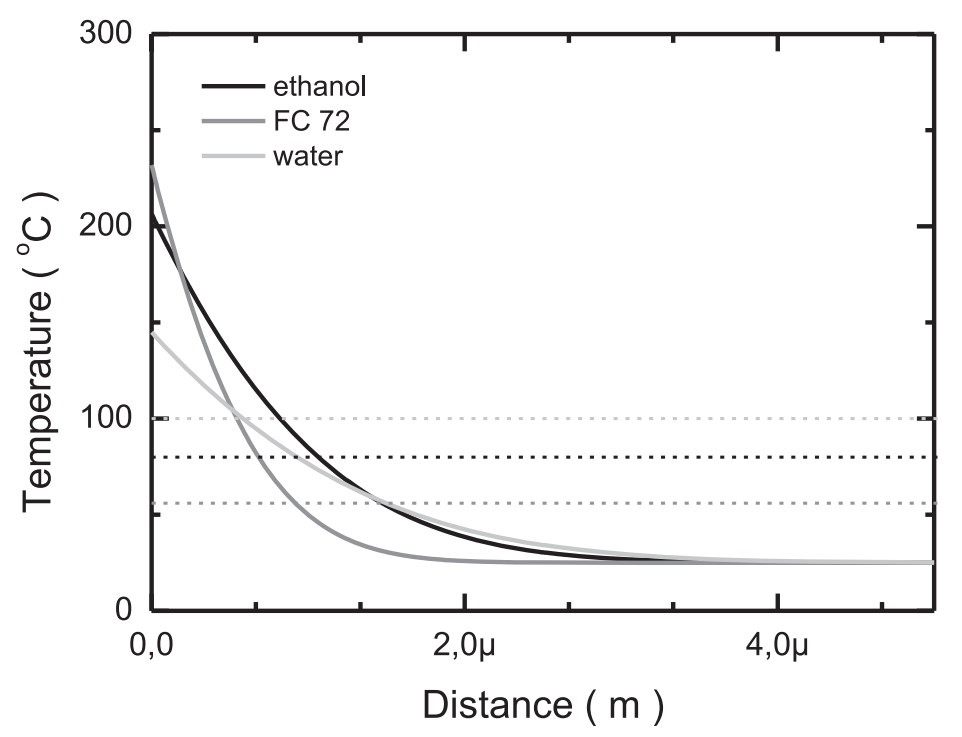

Figure 2.4: Temperature distribution in the liquid (solid line) and the boiling temperature at ambient pressure (dashed line) for ethanol, FC-72 and water.

a temperature higher than the boiling temperature can be transferred into vapour. Of course this is different from what happens in reality, but this gives an example how one can estimate and compare the initial bubble volume. The boiling temperature of FC-72 at ambient pressure is $56^{\circ} \mathrm{C}$. This temperature is reached at a distance of $920 \mathrm{~nm}$ from the heater. The layer of liquid between this point and the heater surface has a temperature above the boiling temperature and will be turned into vapour. For water with a boiling temperature of $100^{\circ} \mathrm{C}$ this distance is only $580 \mathrm{~nm}$ and for ethanol this distance is $1,1 \mu \mathrm{m}$. The initial bubble will be a vapour sheet with an area of the heater multiplied and the height equal to this distance. The volume of the bubble will be equal to the product of the area and this distance. The largest volume of vapour is thus generated for ethanol and this volume is twice as large as the volume of water vapour created under similar heating conditions. Again it must be stated that this is different from reality. A heater temperature of $220^{\circ} \mathrm{C}$ will never be reached without bubble nucleation if FC-72 is used as a liquid. The bubble nucleation will take place earlier and the volume will be smaller. Under similar heating conditions water can be heated to a much higher temperature before nucleation occurs. If the heating is continued the bubble nucleation will take place at a later stage and the volume will be larger than estimated in here.

The one-dimensional model can also be used to get an idea about the temperature profile after the heating pulse has stopped. If we change the boundary conditions in equation (2.6) to

$$
\begin{aligned}
T & =T_{0} \text { at } t=0 \text { and } z=\infty \\
-\left.k_{l} \frac{\partial T}{\partial z}\right|_{z=0^{+}}+\left.k_{s} \frac{\partial T}{\partial z}\right|_{z=0^{-}} & =q \text { for } 0 \leq t \leq t_{\text {pulse }} \\
-\left.k_{l} \frac{\partial T}{\partial z}\right|_{z=0^{+}}+\left.k_{s} \frac{\partial T}{\partial z}\right|_{z=0^{-}} & =0 \text { for } t>t_{\text {pulse }}
\end{aligned}
$$


where $t_{\text {pulse }}$ is the pulse length, we get equation (2.7) during the heating pulse. For $t>t_{\text {pulse }}$ we get

$$
\begin{aligned}
T= & \frac{q}{\frac{k_{s}}{\sqrt{D_{s}}}+\frac{k_{l}}{\sqrt{D_{l}}}}\left[2\left(\sqrt{\frac{t}{\pi}} e^{-\frac{z^{2}}{4 D_{l} t}}-\sqrt{\frac{t-t_{\text {pulse }}}{\pi}} e^{-\frac{z^{2}}{4 D_{l}\left(-t_{\text {pulse }}\right)}}\right)\right. \\
& \left.-z\left(\frac{\operatorname{erfc}\left(\frac{\mathrm{z}}{\sqrt{4 \mathrm{D}_{1} \mathrm{t}}}\right)}{\sqrt{D_{l}}}-\frac{\operatorname{erfc}\left(\frac{\mathrm{z}}{\sqrt{4 \mathrm{D}_{1}\left(\mathrm{t}-\mathrm{t}_{\text {pulse }}\right)}}\right)}{\sqrt{D_{l}}}\right)\right]+T_{0}
\end{aligned}
$$

From this formula the temperature profile in the liquid can be calculated. By interchanging the liquid and solid properties the temperature profile in the substrate can also be determined.

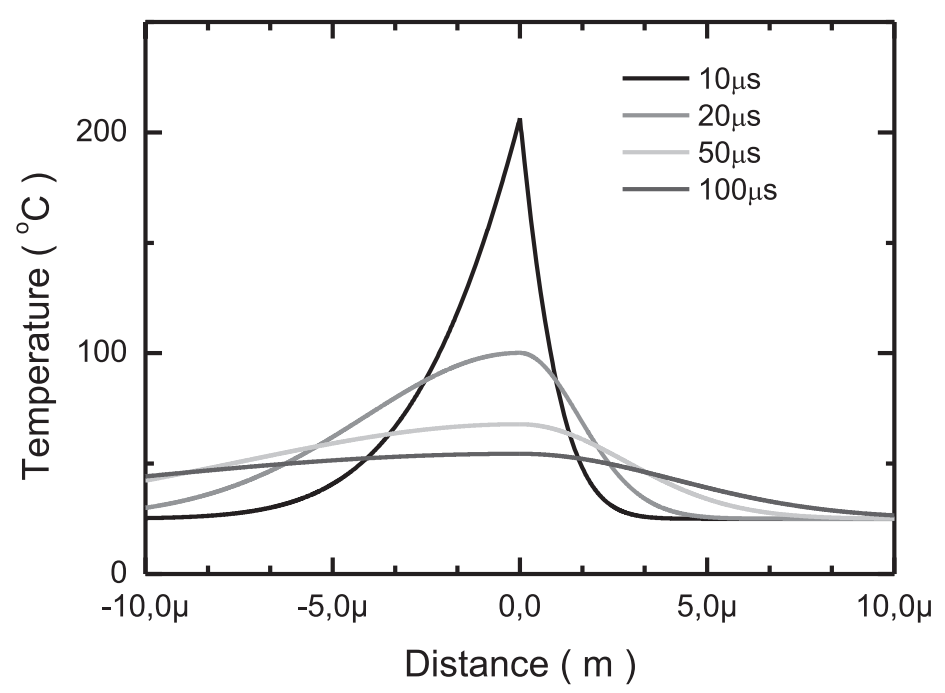

Figure 2.5: Temperature distribution in the pyrex substrate (negative z-axis) and in ethanol (positive axis) at 10, 2050 and $100 \mu \mathrm{s}$ after the start of the heating pulse. The heating pulse has a duration of $10 \mu \mathrm{s}$ and a heat-flux of $100 \mathrm{MW} / \mathrm{m}^{2}$.

Figure 2.5 shows the heater profile for a $10 \mu \mathrm{s}$ heating pulse at a $100 \mathrm{MW} / \mathrm{m}^{2}$ heat-flux in ethanol. The negative distance corresponds to the solid part and the positive distance corresponds to the liquid. The heating pulse stops at $10 \mu \mathrm{s}$ and after this the heater starts to cool down. The liquid as well as the solid close to the heater also start to cool down. Further away the temperature of the liquid can still increase for some time. From the difference in temperature gradient one can also see that the heat penetrates much further into the solid than into the liquid. The bigger area underneath the curve for the solid part shows that more energy goes into the solid part than to the liquid part. Here again the diffusivity of the different materials determines the amount and the depth of heat penetration. A large diffusivity results in a relatively large heated volume, but often also to a slower decrease in temperature after the heating pulse, if only diffusion is considered. In reality, however, a bubble will be formed during the heating pulse and the formation, growth and collapse will have a large influence on the heat-transfer. The presence of a 
fast growing and collapsing bubble will result in local convection. The effects of this local convection will enhance the heat-transfer from the heater to surrounding liquid. The heater temperature itself will quickly start to increase at the moment a bubble is situated on the heater during the heating pulse, due to a much lower thermal conductivity of the vapour phase in comparison to the liquid phase. If a bubble is situated on the heater after the heating pulse, this also will result in a much slower decrease in temperature during the cooling period.

\subsubsection{The 2-D conduction model}

The one dimensional model can only be used in cases where the heat penetration depth is much smaller than the width and length of the heater. In this case the heat transfer parallel to the heater surface can be neglected. If the distance of heat penetration reaches values close to the heater length or width the heat transfer parallel to the heater surface must also be considered. Especially during the cooling period and at relatively low heatfluxes the influence of this heat-transfer will become important. Although analytical solutions of the heater temperature for the two and three dimensional heat conduction problem do exist [80, 68], a numerical simulation can also give a good estimate of the heater temperature. Furthermore a numerical simulation can be used to calculate the temperature distribution in the liquid and substrate. It is possible to produce a three dimensional model of the exact heater structure and its surroundings and obtain a highly accurate temperature profile. This will, however, take a considerable amount of time and computing power. Therefore two much simpler two dimensional models will be considered.

The Cartesian model can be used for heaters with a much longer length than width. The heat-transfer parallel to the heater surface in the direction along the length of the heater is neglected. A heater with a width of $50 \mu \mathrm{m}$ on a $500 \mu \mathrm{m}$ wide and $500 \mu \mathrm{m}$ thick substrate covered by a $500 \mu \mathrm{m}$ thick liquid layer of ethanol is considered for this model. The temperature at the boundaries is constant at $25^{\circ} \mathrm{C}$ and the liquid as well as the substrate are initially at $25^{\circ} \mathrm{C}$. In the Cartesian 2-D model it is assumed that the heater length is still much larger than heat-penetration depth. For heaters with a much larger length than width this assumption is correct, but for a heater where the length and width are comparable the situation is different. In these cases a two dimensional model with an axial symmetry is more suitable. In the axi-symmetrical model the substrate and the liquid are considered to be cylinders both with a height of $500 \mu \mathrm{m}$ and a radius of $250 \mu \mathrm{m}$. The heater is a circular surface with a radius of $25 \mu \mathrm{m}$ situated at the interface between the liquid and the substrate. The centre of the heater is equal to the centre of the cylinders.

\section{Short heating pulses}

The micro-bubble actuator uses short heating pulses of several microseconds to heat the surrounding liquid. In the previous section the temperature in the liquid and the surface were estimated from the analytical solution of the 1-D conduction model. This problem was solved numerically under identical conditions for 2-D Cartesian conduction model. Figure 2.7a shows the temperature profile after a $10 \mu$ s pulse with a heat flux of $1 \times 10^{8} \mathrm{~W} / \mathrm{m}^{2}$. Only a small part of the liquid is heated by the heating pulse. Therefore 
figure $2.7 \mathrm{~b}$ shows a zoom-in on the area close to the heater. The heat penetrates slightly further into the substrate(downward) than in the liquid(upward). The temperature distribution along the width of the heater surface is shown in figure 2.6a. For a large part of the heater surface the temperature is equal to $205^{\circ} \mathrm{C}$, but at the edges the temperature quickly drops. In this case the temperature starts to drop at a distance of about $20 \mu \mathrm{m}$ from the centre of the heater.

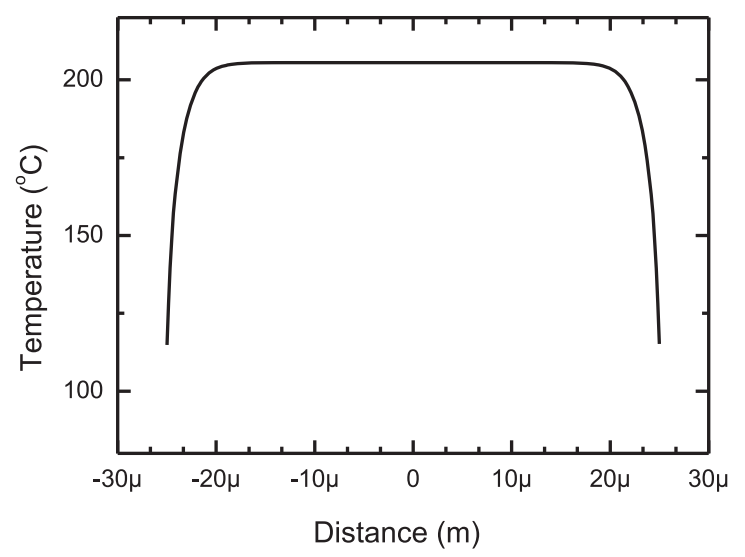

(a) Heater temperature

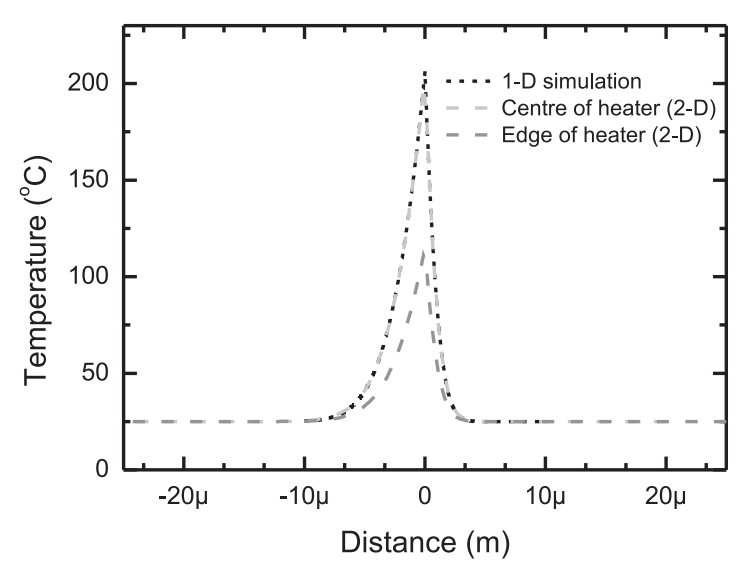

(b) Cross section

Figure 2.6: Local heater temperature and the temperature of the liquid and the substrate along a cross-section through the middle of the heater, at the edge of the heater and results for the 1-D model. after a $10 \mu \mathrm{s}$ heating pulse for a $50 \mu \mathrm{m}$ wide heater.

Figure 2.6b shows the temperature distribution in the substrate and the liquid at the end of the heater pulse. The temperature is shown along cross-sections perpendicular to the heater surface through the centre of the heater as well as the edge of the heater. The temperature distribution in the centre is equal to the distribution found for the onedimensional model. The temperature near the edge of the heater is always lower than the temperature at the centre part of the heater.

At the end of a short heating pulse a difference in temperature distribution and heat penetration is only present near the edges of the heaters. The heater temperature as well as the temperature of the liquid and substrate close to the edge is always lower. For a large part of the heater, about $80 \%$ of its total width, the temperature distribution and heat penetration into the substrate and the liquid are nearly identical to the results found for the one-dimensional model. For these short heating pulses the 1D model can thus still be used to predict the temperature and temperature distribution in the substrate and the liquid without large errors.

\section{Cooling}

After the heating pulse has ended, the systems cools down. After $1 \mathrm{~ms}$ the thermal penetration length reaches values comparable to the heater length and the results of the 1-D model will start to deviate considerably. A 2-D model will give a much better representation. 


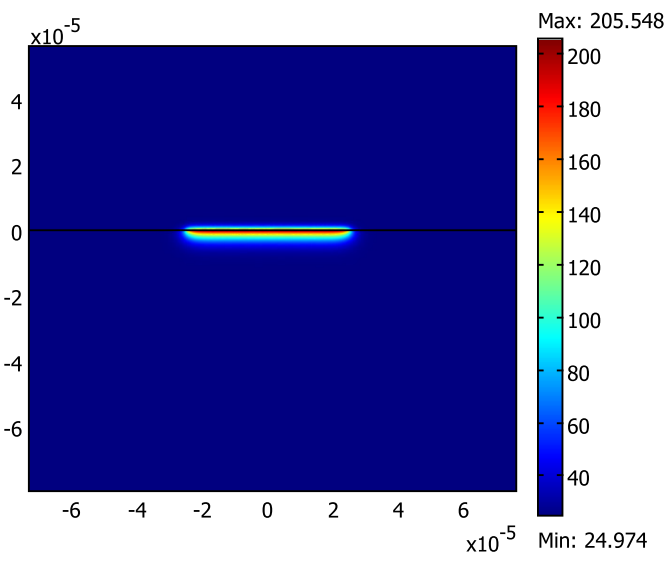

(a) Temperature profile

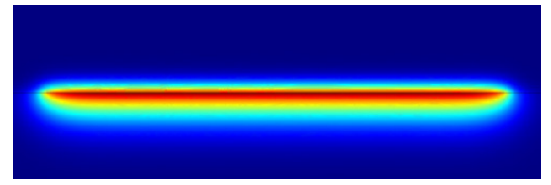

(b) Zoom-in

Figure 2.7: Temperature profile $(y-z)$ and a zoom-in on the area close to the heater after a $10 \mu$ s heating pulse for a $50 \mu \mathrm{m}$ wide heater.

Figure 2.8a shows the temperature distribution after $1 \mathrm{~ms}$ for the 2-D Cartesian model. The temperature of the liquid and the substrate close to the heater is substantially lower than just after the heating pulse (figure 2.7). The heat penetration depth, however, is much larger. The energy is distributed over a larger volume of the liquid and the substrate. The energy is spreads in all directions and this results in a more cylindrical temperature profile. The highest temperature can be found in the liquid about $4 \mu \mathrm{m}$ above the centre part of the heater. The heat penetration into the substrate is much further than into the liquid.

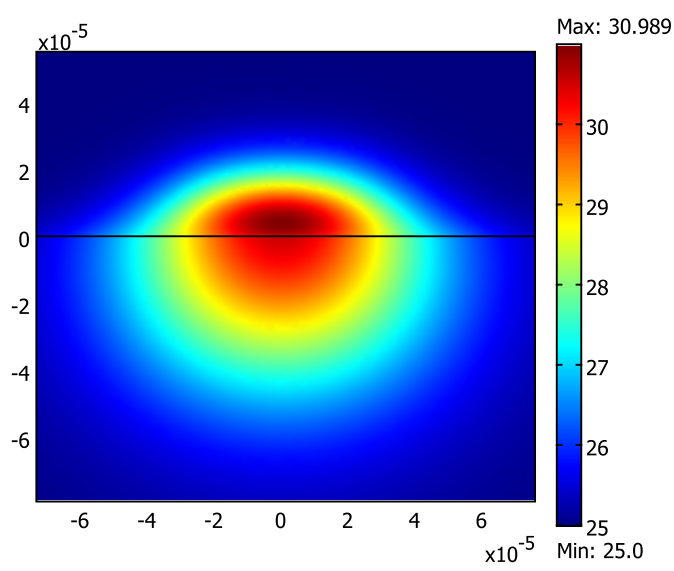

(a) Temperature profile

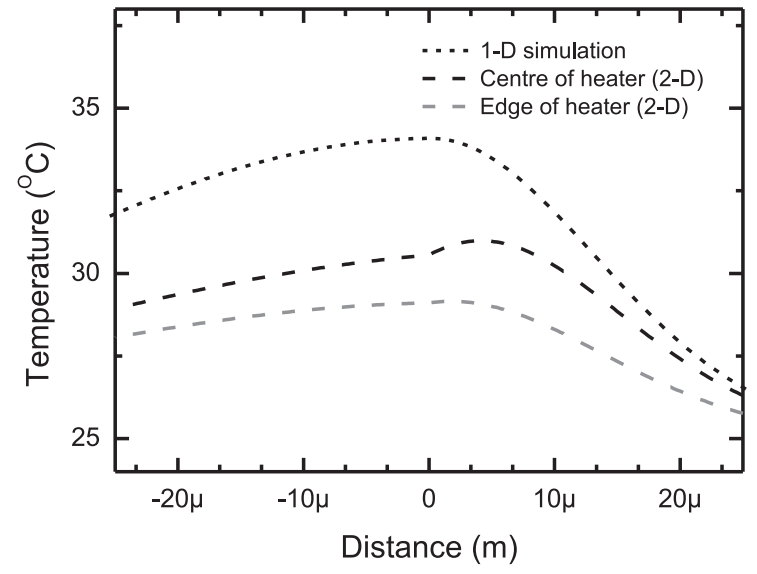

(b) Distribution in the z-direction

Figure 2.8: Temperature profile $(y-z)$ and temperature distribution at the centre and at the edge of the heater after a cool down period of $1 \mathrm{~ms}$ for a $50 \mu \mathrm{m}$ wide heater. In (b) the temperature distribution for the 1-D model is shown for comparison.

Figure $2.8 \mathrm{~b}$ shows the temperature distribution across the centre and the edge of the heater. The results for the one-dimensional model are also shown in the figure. Unlike 


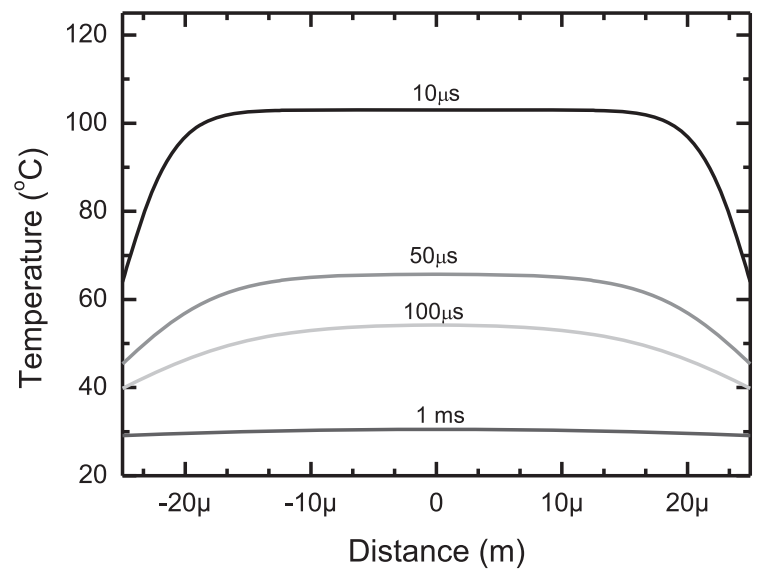

(a) Heater temperature

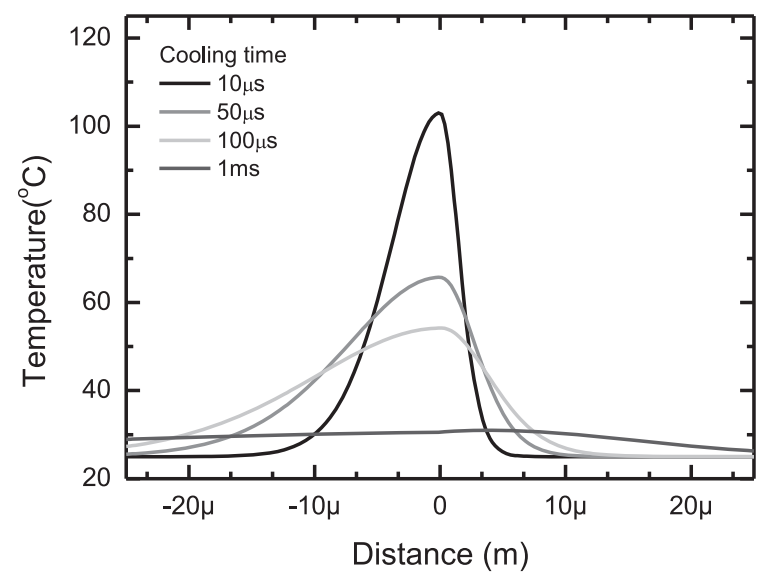

(b) Temperature distribution in $z$-direction

Figure 2.9: Temperature distribution at the heater surface $(z=0)$ and temperature distribution across a cross-section in the z-direction through the centre of the heater after several different cooling times.

the situation at the end of the heating pulse shown in $2.6 \mathrm{~b}$ there is a large difference in the distribution between the one-dimensional model and the two dimensional model. The temperature for the two dimensional model is much lower over the entire range and the highest temperature can be found in the liquid instead of on the heater surface. The difference in temperature between the edge of the heater and the middle part is much smaller than at the end of the heating pulse.

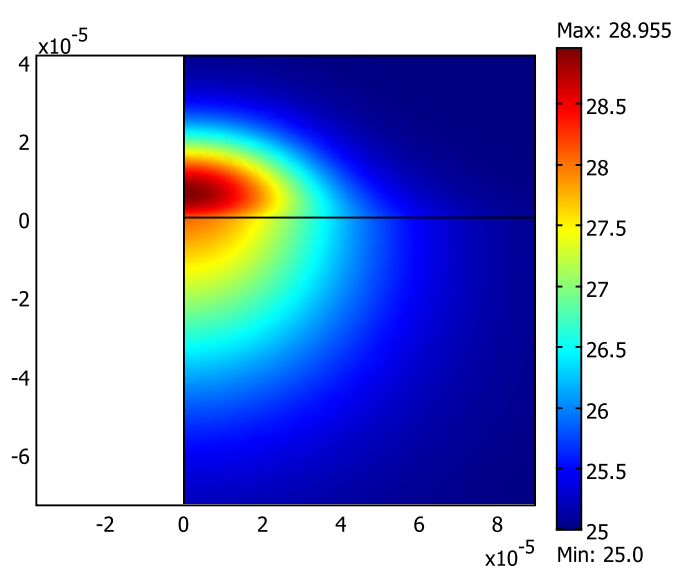

(a) Temperature profile

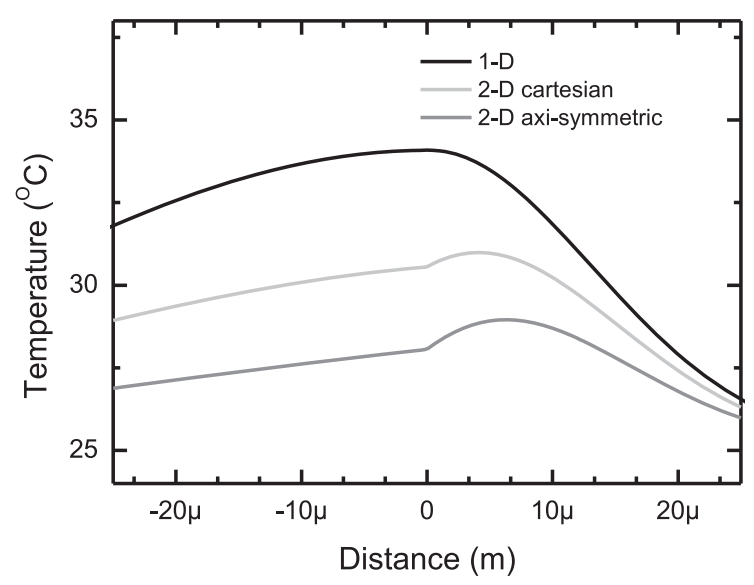

(b) Temperature distribution

Figure 2.10: Temperature profile $(r-z)$ for the 2-D axi-symmetrical model and temperature distribution in the $z$-direction at $r=0$ or $y=0$ for the 3 different models after a cool down period of $1 \mathrm{~ms}$.

Figure 2.9a shows the heater temperature for several different cooling times and figure $2.9 \mathrm{~b}$ shows the corresponding temperature distribution across a cross-section in the 
z-direction through the centre of the heater. With increasing cooling times a larger part of the heater has a lower temperature than the central part of the heater. The difference in temperature between the middle of the heater and the edges however decreases with cooling time. The temperature distribution at first sight looks quite similar to the distribution shown in figure 2.5, but figure $2.8 \mathrm{~b}$ has shown that there are differences.

For heaters with a geometry for which the length and width are comparable the 2-D axi-symmetric model will give a better representation. Figure 2.10a shows the temperature profile of a cross-section in the z-direction parallel to rotational axis and at the centre of the heater for the 2-D axi-symmetrical model under similar heating conditions and cooldown time of $1 \mathrm{~ms}$. Similar to the 2-D Cartesian model the highest temperatures can be found in the liquid with the maximum located at several micrometers above the heater surface. A larger volume of the substrate in comparison to the liquid has an elevated temperature. The profile looks quite similar to the profile found for the 2-D Cartesian model shown in figure 2.8a. In order to make a good comparison between the different models the temperature distribution after a $1 \mathrm{~ms}$ period of cooling along the $\mathrm{z}$-axis for the 2-D axisymmetrical model, the 2-D Cartesian model and 1-D model is shown in figure 2.10b. For all the models a $10 \mu$ s heating pulse and an identical heating power corresponding to $1 \mathrm{x} 10^{8} \mathrm{~W} / \mathrm{m}^{2}$ were used in the simulation. The temperature for the $2-\mathrm{D}$ axi-symmetrical model shows a similar behaviour as the Cartesian model, but the temperatures are several degrees lower and the maximum temperature is located at a distance of $6,4 \mu \mathrm{m}$ from the heater heater surface located at $0 \mu \mathrm{m}$.

In the axi-symmetrical model diffusion can take place in three directions. This will result in an even further increase of the average diffusion speed and thus a faster cooling of the heated substrate, liquid and heater surface. For all the models the diffusion of heat into the substrate is faster than in the liquid. The heated volume of the substrate is thus always bigger than the heated volume of the liquid. For the 2-D models a volume of liquid with a temperature above the heater or substrate temperature develops during cool down. The maximum temperature and distance from the heater surface depend on the model. For the axi-symmetrical model the maximum temperature is lower and the distance between the point with the maximum temperature and the heater is larger than for the Cartesian model.

The difference between the one and two dimensional models is relatively small during and at the end of short heating pulses. Nevertheless after the heating stops and the heater starts to cool down, differences start to appear between the models. In the two dimensional models the diffusion can take place perpendicular as well as parallel to the heater surface. There is no preferred direction for the diffusion of heat and it will spread equally in all directions. In most cases the diffusion of heat from a heated surface is also increased. The temperature of the heater itself and the liquid and the substrate close to the heater will drop faster. The diffusion of heat through the liquid is slower than through the substrate and after some time the highest temperature can be found in the liquid. In the temperature-profile it can be seen that a spherical area with an elevated temperature develops at some distance above the heater. The temperature at the edges of the heater is always lower than the temperature at the centre of the heater, but this difference decreases with cooling time. Furthermore the part of the heater with a temperature almost equal to the temperature at the centre of the heater also decreases with cooling time. The 
temperature profile across the heater becomes more rounded. This is an indication that the one-dimensional model is not valid for long cooling times.

\section{Long heating pulses}

For short and intensive heating pulses the one dimensional model gives a good representation of the heater temperature during heating for a large part of the heater. If a longer heating pulse with a lower heating power is used, the 1D model gives a less accurate representation of the system and a $2 \mathrm{D}$ dimensional model will give a better result.

Figure 2.12a shows the temperature profile after a simulation for the 2-D Cartesian model used earlier. In this case the heating power is lower, at $1.6 \times 10^{7} \mathrm{~W} / \mathrm{m}^{2}$ and the heating time must be increased to $500 \mu \mathrm{s}$ to reach the same temperature as for the short high heat-flux heating pulses. When compared to the short heating pulses the volume with a temperature above ambient is much larger and the temperature profile is more rounded. The temperature-distance curve of the heater surface shown in figure $2.12 \mathrm{~b}$ is also more rounded.

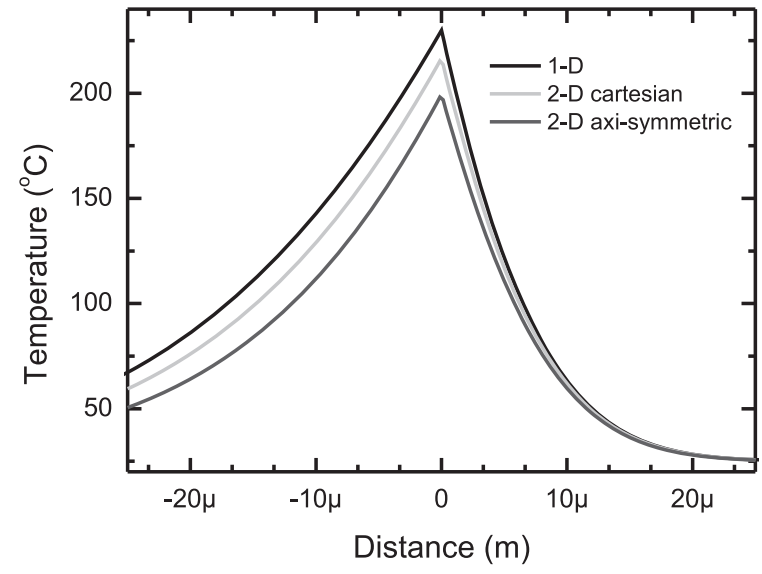

(a) End of heating pulse

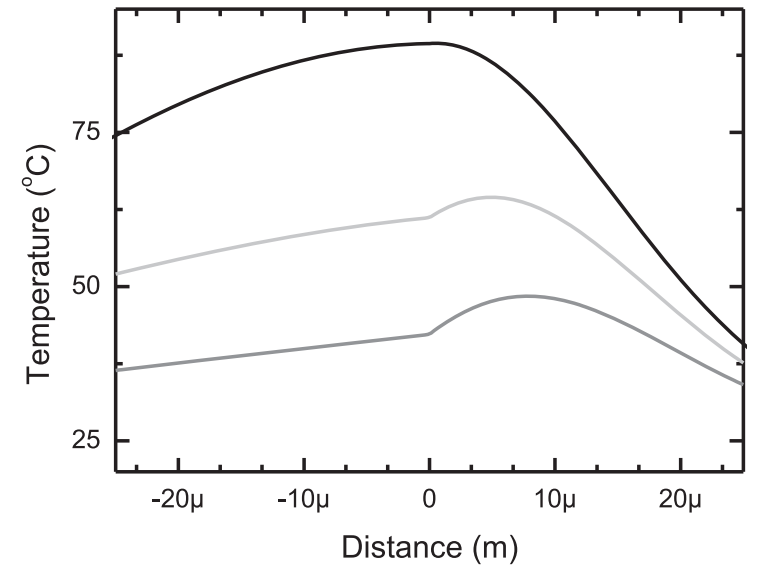

(b) After $1 \mathrm{~ms}$ cool down

Figure 2.11: Temperature distribution along the $z$-axis at $y=0$ or $r=0$ at the end of a $500 \mu \mathrm{s}$ pulse with a heating power of $1.6 \times 10^{7} \mathrm{~W} / \mathrm{m}^{2}$ and after a cooling period $1 \mathrm{~ms}$ for the three different models.

In figure 2.11 a comparison between the three different models for the relatively long and low power heating pulse is made. Figure 2.11a shows the temperature distribution in the z-direction at the end of the heating pulse. The highest temperature is found at the heater surface for the 1-D model. The temperature difference in the liquid region is much smaller than in the substrate. The difference in temperature distribution for the three simulation models after a $1 \mathrm{~ms}$ cooling period (figure $2.11 \mathrm{~b}$ ) is much larger. The figure looks similar to figure $2.10 \mathrm{~b}$, but the absolute temperatures as well as the temperature differences between the models are much larger.

For relatively low power heating pulses the pulse time must be increased to reach the same temperature as for short heating pulses. This increase in pulse length leads to a deeper penetration of heat into the liquid and a cylindrical temperature profile in the 
liquid and substrate as well as on the heater surface. Different models result in different temperatures at the end of the heating pulse. The 1-D dimensional model clearly can not be used any more and it will depend on the heater geometry, which of the two twodimensional models give the best representation.

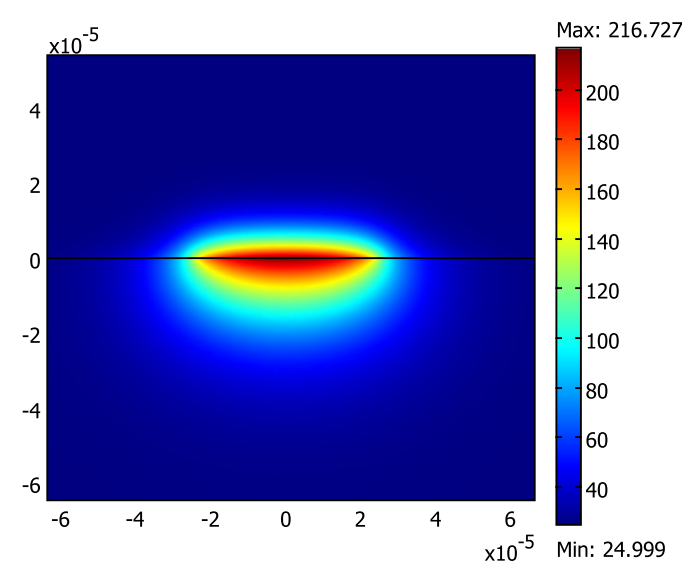

(a) Temperature profile

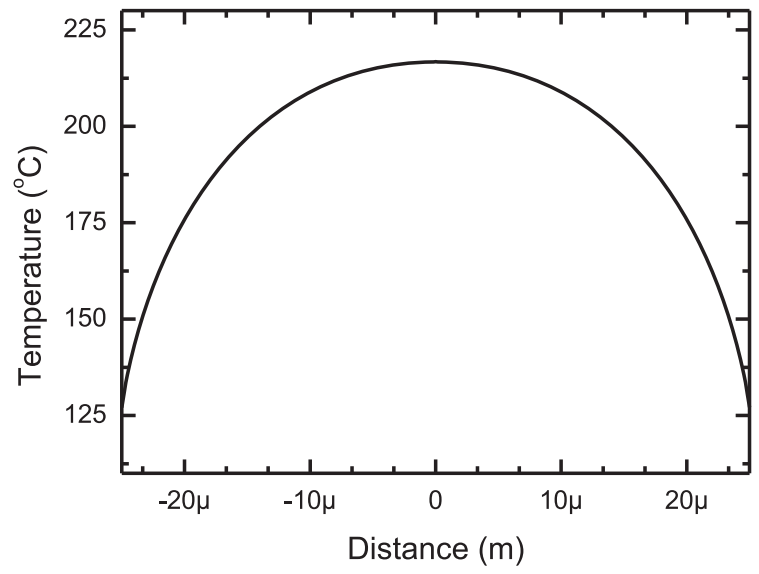

(b) Heater temperature

Figure 2.12: Temperature profile $(y-z)$ and heater temperature $(z=0)$ after a $500 \mu$ s pulse with a heating power of $1.6 \times 10^{7} \mathrm{~W} / \mathrm{m}^{2}$.

After a cooling period of $1 \mathrm{~ms}$ the temperature distribution for all the models look similar to the distribution after a cooling period of $1 \mathrm{~ms}$ for the short heating pulses. The temperatures however are much higher and the temperature maximum lies at a slightly larger distance from the heater. After a long heating pulse it will take longer to cool the system down and a larger part of the system will be effected by heating pulse.

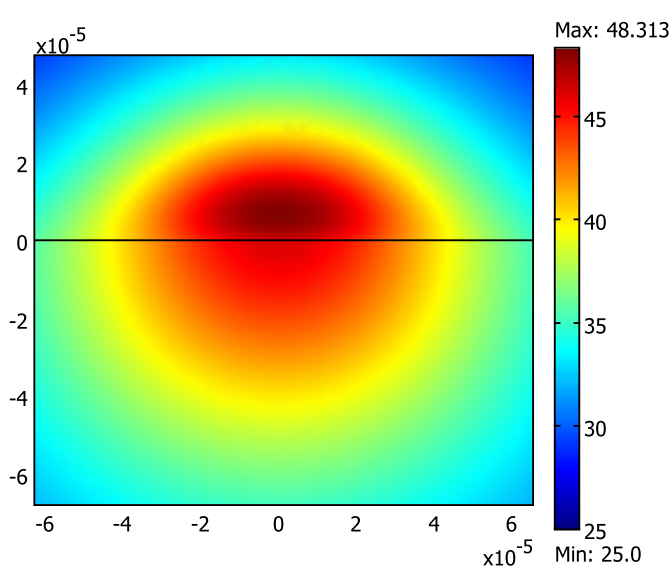

(a) Temperature profile

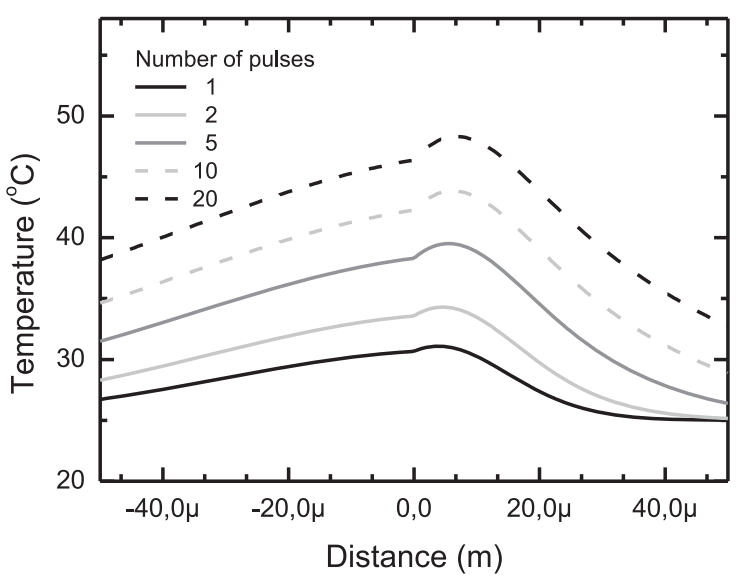

(b) Temperature distribution

Figure 2.13: Temperature profile $(y-z)$ after 20 pulses and the temperature distribution along the $z$-axis through the centre of the heater $(y=0)$ after a number of pulses. 


\subsubsection{Repetition frequency}

In the previous sections the effect of a single heating pulse on the temperature was considered. The micro-bubble actuator, however, is intended to operate at frequencies up to several kHz. This corresponds to more than thousand pulses per second and a time between pulses of less than $1 \mathrm{~ms}$. Figures $2.10 \mathrm{~b}$ and $2.12 \mathrm{~b}$ have shown that the heater surface as well as a part of the liquid and substrate are still at temperatures above ambient after a $1 \mathrm{~ms}$ cooling period. A new heating pulse will result in a quick temperature increase of the heater and its surroundings. If the pulse length and heat-flux are identical to the previous pulse a higher heater temperature will be reached at the end of the second heating pulse and the system will heat up.

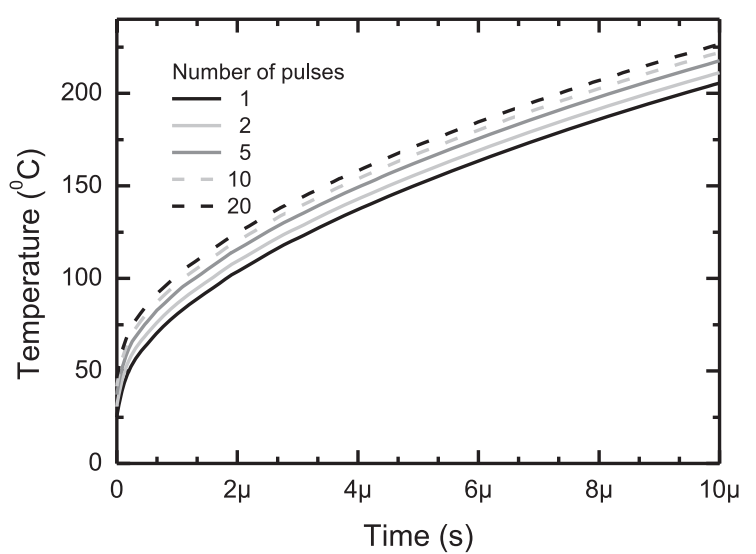

(a) Heating

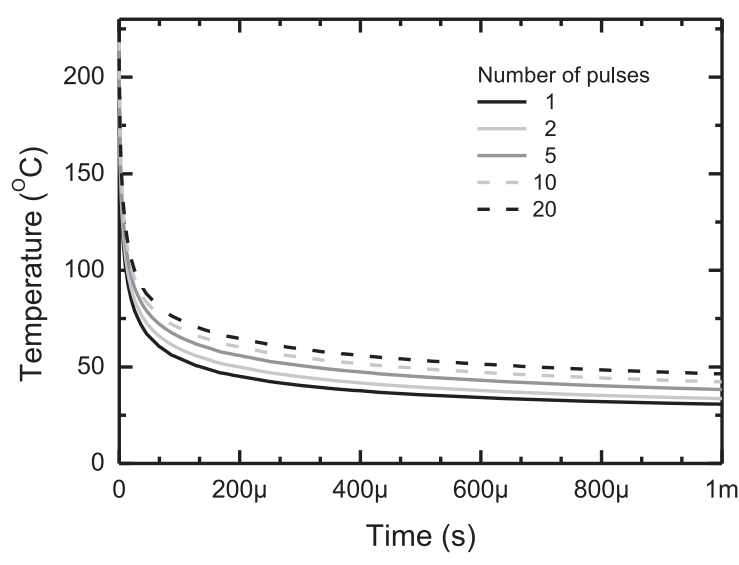

(b) Cooling

Figure 2.14: Heater temperature $(y=0, z=0)$ during a $10 \mu$ s pulse with a heating power of $1 \times 10^{8} \mathrm{~W} / \mathrm{m}^{2}$ and during cooling period for a number of repetitions.

The Cartesian 2-D model is used to investigate the effect of heating by a number of pulses. The length of the heating pulses is $10 \mu \mathrm{s}$ and the heat-flux is $1 \times 10^{8} \mathrm{~W} / \mathrm{m}^{2}$. The conditions are thus identical to the ones used for the single pulses. After each pulse a $1 \mathrm{~ms}$ long cooling period was used. Figure 2.14a shows the heater temperature during the heating pulses and figure $2.14 \mathrm{~b}$ shows the heater temperature during cooling. The curve for one pulse is identical to the curve found in the previous section for the single pulse. As the number of pulses increase the temperature during heating as well as cooling increases over the entire range of time. On average the heater increases in temperature. The amount of increase, however, decreases with the number of pulses. This is made more clear in figure 2.15.

Figure $2.15 \mathrm{~b}$ shows the heater temperature at the end of the heating pulse as a function of the number of pulses and figure 2.15a shows the heater temperature at the end of the $1 \mathrm{~ms}$ cooling period. The temperature at the end of the heating pulse increases from $206^{\circ} \mathrm{C}$ after 1 pulse to $227^{\circ} \mathrm{C}$ after 20 pulses. The temperature at the end of the cooling period and thus at the start of the next heating pulse increases from $31^{\circ} \mathrm{C}$ after one pulse to $46^{\circ} \mathrm{C}$ after 20 pulses. For both curves the slope of the temperature-time curve decreases with the number of pulses. A situation close to a steady state will thus be reached after 


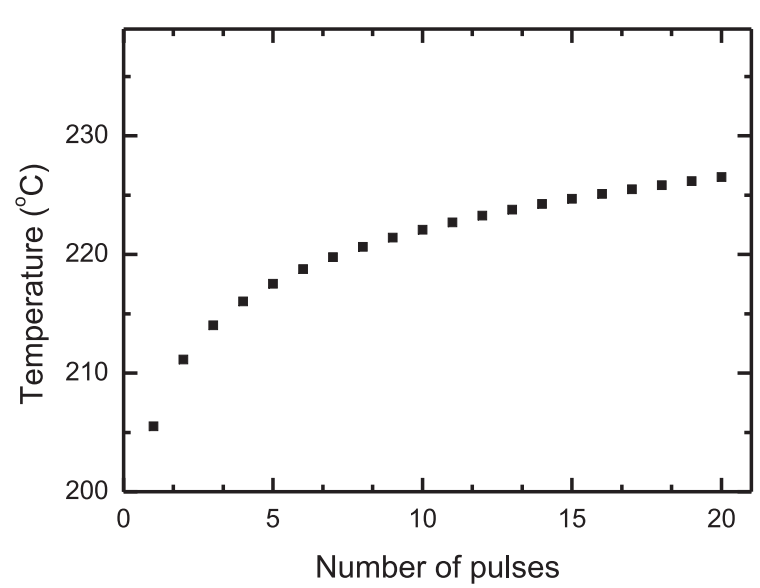

(a) End of heating pulses

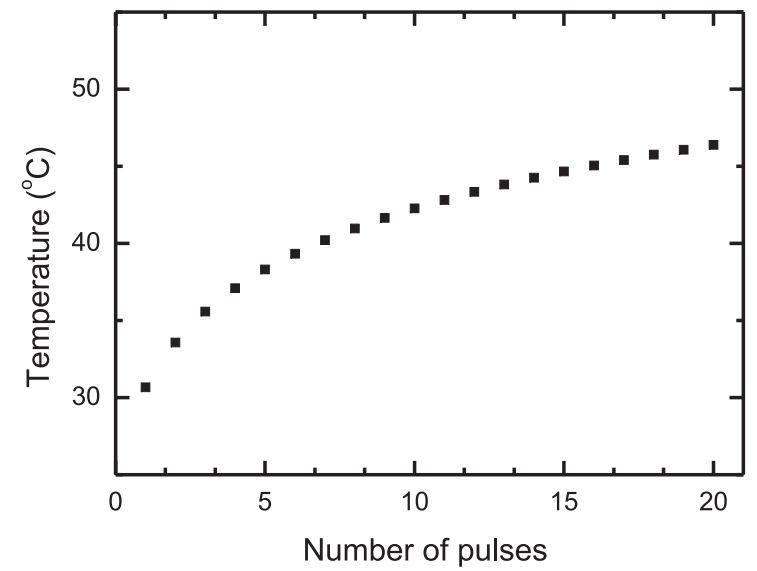

(b) End of cooling period

Figure 2.15: Heater temperature at the end of a $10 \mu$ s heating pulse of $1 \times 10^{8} \mathrm{~W} / \mathrm{m}^{2}$ and after a 1ms cooling period as a function of the number of pulses.

a number of pulses and the temperatures at the start and at the end of the heating pulse will become nearly constant. However, during a single pulse, the temperature increase stays the same and the difference in temperature between the start and the end of the heating pulse will be around $180^{\circ} \mathrm{C}$.

After 20 pulses the temperature profile at the end of the cooling period(figure 2.13a) looks similar to the profile after one pulse(figure 2.8a). Nevertheless the temperatures are much higher and a larger volume has a temperature above the ambient temperature of $25^{\circ} \mathrm{C}$. Furthermore, the cylindrical volume with a temperature above the heater temperature is much bigger. Figure 2.13b shows the temperature distributions of a cross-section in the z-direction through the centre of the heater after a different number of pulses. The temperature increases with the number of pulses. After the first pulse the liquid is still at ambient temperature for distances of $40 \mu \mathrm{m}$ and further from the heater. One pulse later this distance already has increased to nearly $50 \mu \mathrm{m}$.

The location and value of the maximum temperature changes with the number of pulses. Figure 2.16a shows the distance to the heater from the location of the temperature maximum. This distance increases with the number of pulses and again the slope of the curve decreases with number of pulses. The distance between the heater and the location of the maximum temperature is only several micrometers under these conditions. The maximum temperature is shown in figure 2.16b. The maximum temperature increases with the number of pulses and the slope of the temperature curve decreases. The heater temperature, which was already shown in figure $2.15 \mathrm{~b}$, is also shown in this graph. As the number of pulses increases the difference between the maximum temperature and the heater also increases, albeit only a few degrees.

The cooling time $t_{\text {cooling }}$ is directly related to the repetition frequency and equation (2.11) can be used to calculate this time for a particular heating pulse length $t_{\text {pulse }}$ and repetition frequency $f$.

$$
f=\frac{1}{t_{\text {pulse }}+t_{\text {cooling }}}
$$




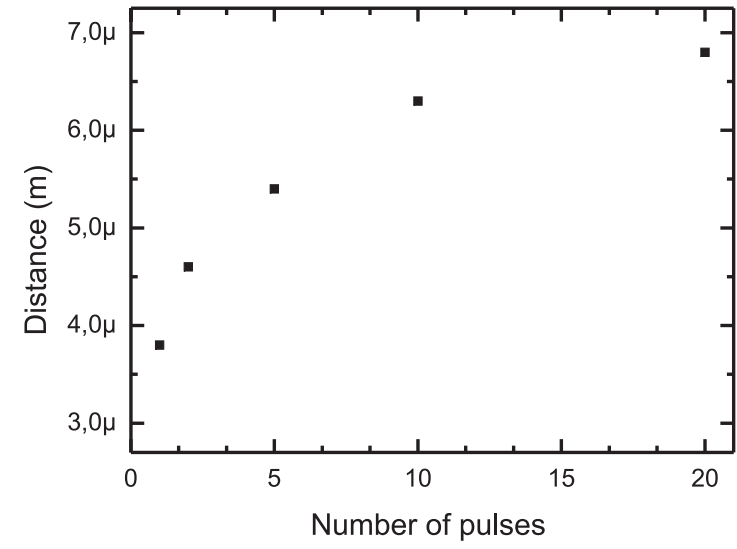

(a) Location

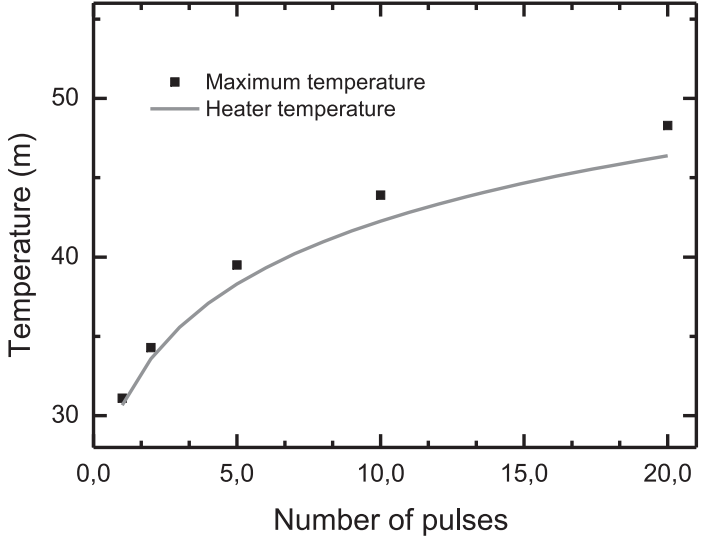

(b) Temperature

Figure 2.16: The distance from heater $(z)$ and temperature of the point of maximum temperature in the liquid as function of the number of pulses. The heater temperature $(z=0)$ is also shown in (b) for comparison.

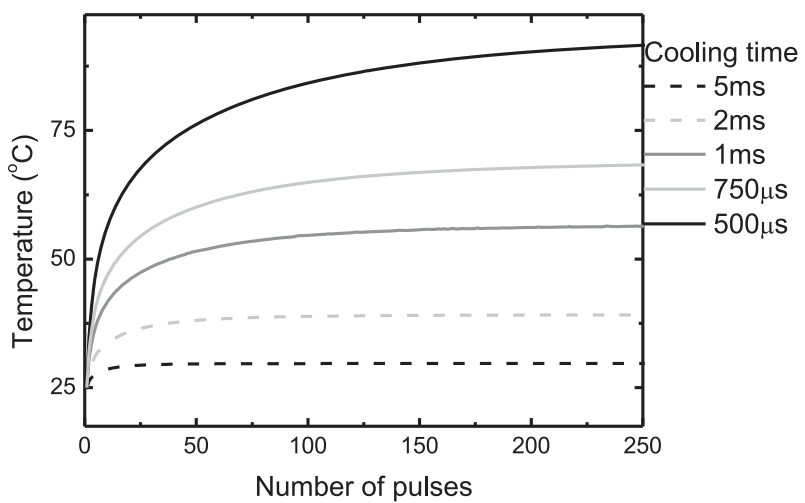

(a)

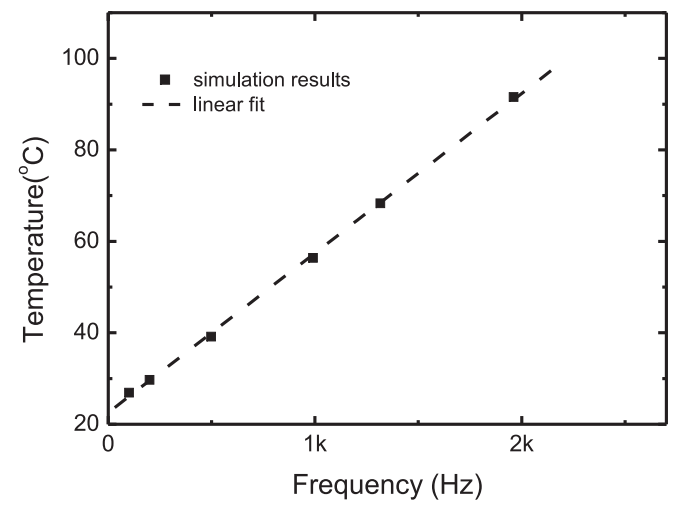

(b)

Figure 2.17: Heater temperature $(z=0)$ as at the start of the heating pulse for 5 different cooling times (a). The heater temperature at the start of the heating pulse as a function of frequency and a linear fit to the measurements. 
Figure 2.17a shows the temperature at the start of the heating pulse as a function of the number of pulses for 5 different cooling times. For all the cooling times the temperature increases quickly during the first pulses. After this fast rise in temperature the increase slows down. For relatively long cooling times of $5 \mathrm{~ms}$ and $2 \mathrm{~ms}$ a constant temperature is reached after 50 and 125 pulses respectively and for a cooling time of $1 \mathrm{~ms}$ it takes 250 pulses to reach a constant temperature. For shorter cooling times a constant temperature at the start is not reached within 250 pulses, and more pulses are needed to reach an equilibrium. The temperature increases with a decreasing cooling time. Figure 2.17b shows the temperature after 250 pulses against the repetition frequency calculated from equation (2.11). The temperature increases almost linearly with frequency in the range between 0 and $2 \mathrm{kHz}$.

In the previous simulations a pulse length of $10 \mu$ s and heat-flux $100 \mathrm{MW} / \mathrm{m}^{2}$ were used. The temperature at the start will also change with the pulse length and the heat-flux. Far away from the heater, the heater can be assumed to be a point source. The steady state temperature distribution will scale linearly with the total heat introduced to the system. In this case a pulsed source is used and the average heat-flux $q_{a v g}$ can be estimated by

$$
q_{\text {avg }}=q f t_{\text {pulse }}
$$

Close to the heater the pulses have a large effect on the temperature distribution, but at some distance from the heater the temperature profile will be determined by the average heat-flux. Here the temperature distribution will thus scale linearly with the heat-flux, frequency and pulse time. The temperature distribution close to the heater will be highly dependent on the heater geometry and time within the period(at the end or begin of a cooling period or heating period, etc.), but figure $2.17 \mathrm{~b}$ shows that the temperature at the start scales linearly with the frequency. If one assumes that this temperature scales similar with the average heat-flux, it will also scale linearly with the heat-flux and pulse time.

\subsubsection{Summary and conclusions on the heat transfer}

The diffusivity of a material determines the speed of heat-transfer through the material. A heating pulse generated in a high diffusivity material will spread fast, but the temperature will increase will be relatively low. At the boundary between two materials the difference in thermal conductivity of the two materials will determine the distribution of the heatflux into the two materials. If the thermal conductivity of the substrate is much larger than the conductivity of the liquid a large part of the produced energy will be 'lost' in the substrate.

For short heating pulses with extremely high heat-fluxes bubble nucleation takes place at temperatures much higher than the normal boiling point of the liquid. The volume of the initial bubble is determined by the volume of liquid heated above the boiling temperature. This volume is directly related to the temperature distribution around the heater. Several models can be used to calculate this temperature distribution. During a high heat flux heating pulse of several microseconds the heat-conduction can be considered one-dimensional for a large part of the heater. The product of the distance between the heater and the isotherm with a temperature equal to the normal boiling temperature 
and the heater area can be used as an estimate of the initial bubble volume at the moment of nucleation. For the short pulses a liquid layer of several micrometers will be heated. After the heating stops the liquid will quickly start to cool down. In this case the one-dimensional model can not be used any more and a 2-D is used to describe the heat-transfer. The exact heater geometry will determine which of the 2-D models, axi-symmetrical or Cartesian, will give the best results. During cooling the distribution profiles become more rounded and heat will spread through the system. Due to its lower conductivity the liquid will cool down slower than the substrate. The highest temperatures will be found in the liquid a few micrometers above the heater surface.

An increase in repetition frequency results in a decrease in cooling time between pulses and the system will start to heat up. The temperature at the start of the heating pulse can be used as an indication of the system temperature. This temperature will increase with repetition frequency. After the heating starts a sort of steady state will be reached after a number of pulses. The number of pulses before a constant temperature at the start of the heating pulse is reached also increase with repetition frequency. The total time it takes to reach this steady state, however, is independent of frequency around $250 \mathrm{~ms}$. The temperature distribution shows a volume above the heater with a temperature higher than the heater temperature. The temperature and the distance of this area from the heater both increase with repetition frequency.

The simulations described in this section only considered heat transfer by diffusion. The simulations give an idea about the influence of heating parameters, such as repetition frequency, length of the heating pulse and applied heat-flux. They can be used to get a good estimate of the heater temperature and the distribution of the temperature in the liquid and substrate before bubble nucleation takes place. The bubble generation and growth, however, have a large effect on the heat-transfer. The heat-transfer from the heater to its surroundings dramatically decreases at the moment a bubble is formed on top of the heater. The thermal conductivity of vapour or gas in the bubble is much lower than the conductivity of the liquid or the substrate. The surface temperature of the heater, therefore, increases much faster after bubble nucleation. The bubble dynamics will also give rise to convection in the liquid, leading to an increase in heat-transfer. The produced heat spreads more quickly through the system, resulting in a faster cool-down. Also at lower heating powers and longer pulse lengths convection might become an important factor in the heat-transfer. Simulations, which also take into account convection effects and bubble formation, are much more complex and will take a considerable amount of time preform.

\subsection{Bubble Generation}

The previous section described the conduction of heat through the liquid. This section will deal with the nucleation of bubbles. The moment in time and space for bubble nucleation depends on many parameters $[42,44]$. Bubbles can only be formed when the liquid temperature is brought above its normal boiling point, the liquid is now superheated. A superheated state can be attained either by heating the liquid or by depressurizing it. A superheated liquid, however, is not the only requirement to get a bubble. First 
a small nucleus needs to be present from which the bubble can grow. There are two main mechanisms for bubble generation. In the first the nucleus is a small vapour or gas bubble trapped in a crevice or defect on the heater or container wall. If the liquid is heated or depressurized this bubble can start to grow. The second mechanism generates bubbles by spontaneous nucleation. Spontaneous nucleation is the process where bubbles are randomly formed in a superheated liquid.

\subsubsection{The phase transition}

A substance can exist in different phases and through a change in pressure or temperature it can change from one phase to another following the rules of thermodynamics. This phase transformation can lead to an abrupt change in one or more physical properties. A phase diagram gives information on the state of a substance at a certain pressure,

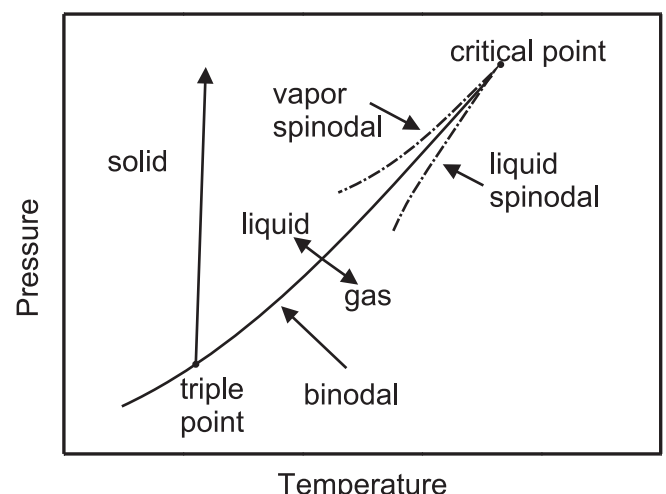

(a) PT diagram

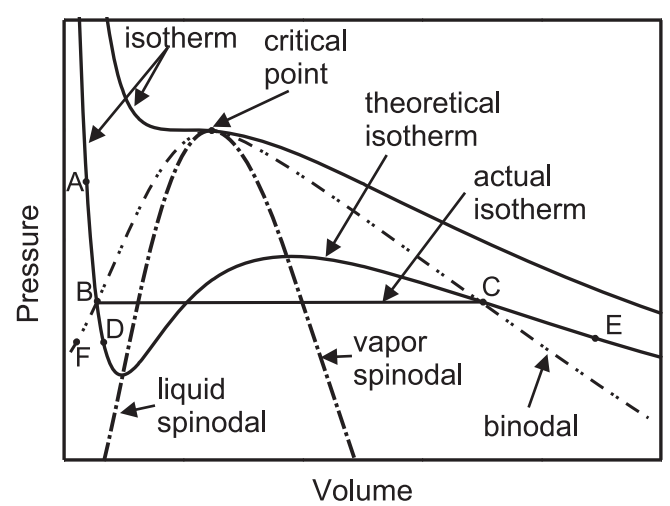

(b) PV diagram

Figure 2.18: Phase diagrams for a simple substance

temperature and specific volume. Figure 2.18 shows P-T diagram and P-V diagram for a simple substance. The triple point is the point in the phase diagram at which the solid, liquid and vapour coexist; the substance has three alternative states. The saturated liquid/vapour line, also called binodal, extends from this point to the critical point. Only on the binodal the liquid and the vapour phase may coexist. Above the binodal the substance is a pure liquid and below the binodal the substance is a pure gas. Above the critical point the difference between liquid and gas is gone and substance is in the fluid phase. Figure 2.18b shows that the binodal has two branches the saturated liquid and the saturated vapour. If one wants to go from the liquid state to the gas state following the theoretical isotherm, this will lead to unstable states. In reality the actual isotherm, which is the horizontal line from $\mathrm{B}$ to $\mathrm{C}$, will be followed. In this case the liquid and vapour phase will coexist during the transition. The line joining the maxima in the theoretical isotherms is called the vapor spinodal line; the line joining the minima is called the liquid spinodal line. Both spinodals end at the critical point.

The region between the spinodal lines and the binodal can be realized under certain special conditions. If, for example, a pure liquid at the state $\mathrm{A}$ is depressureized at 
constant temperature, several things may happen when the pressure is reduced below that of point B (the saturated vapour pressure). If at least one nucleation site is present the liquid will become a vapour as the state moves horizontally from B to C, and at a pressure below the vapour pressure the state will come to equilibrium in the gaseous region at a point such as E. However if no nucleation sites are present, the depressurization may lead to continuation of the state down the theoretical isotherm to a point such as D, called a "metastable state" since imperfections may lead to instability and transition to the point $\mathrm{E}$ [43]. A point like D could also be reached by proceeding along an isobar from a point such as $\mathrm{F}$ by increasing the temperature. The state $\mathrm{D}$ is now called superheated. The maximum superheat at a certain pressure is equal to the difference between a binodal and the liquid spinodal, this value decreases with an increase in pressure and at the critical point the value is zero. This maximum is also called the thermodynamic limit of superheat.

\subsubsection{Kinetic limit of superheat}

The Gibbs energy of a system is a thermodynamic potential which measures the "useful" work obtainable from an isothermal, isobaric thermodynamic system. In a stable equilibrium state of the system the Gibbs energy is at a minimum. If, for example, the system is a fluid, which can be in gas or liquid phase, the phase with the lowest Gibbs energy will be the only stable state. The two states can only coexist under conditions were the Gibbs energy and the chemical potential of both phases are identical.

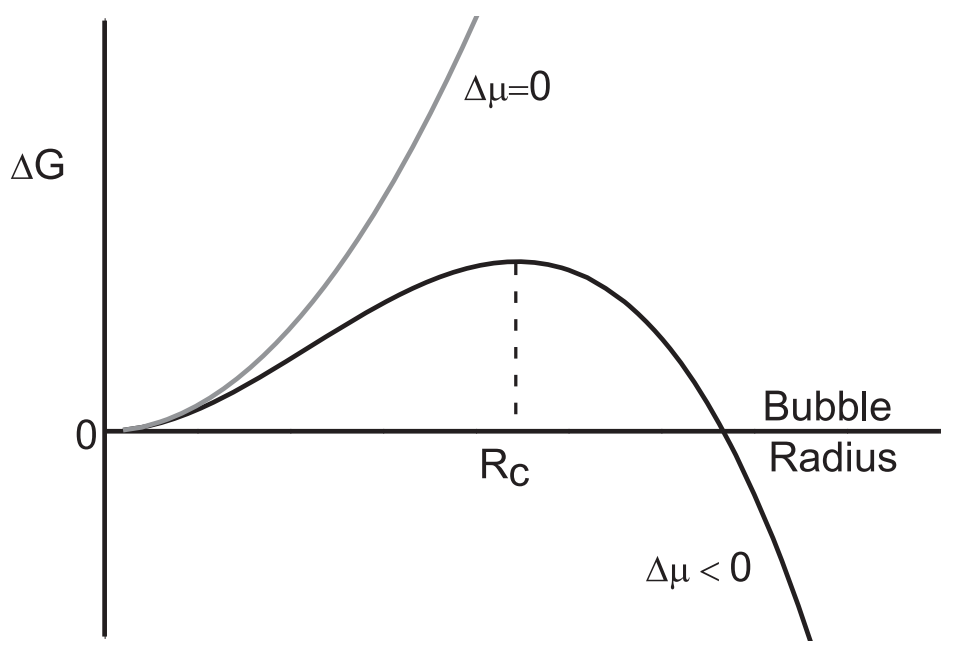

Figure 2.19: Schematic of the difference in Gibbs energy between the liquid and gas phase against the bubble radius for $\Delta \mu=0$ and for $\Delta \mu<0$

If a gas bubble is created in a liquid the difference in Gibbs energy $(\Delta G)$ between the gas and the liquid state is given by:

$$
\Delta G=4 \pi R^{2} \sigma+\frac{4 \pi}{3} R^{3} n_{g} \Delta \mu
$$

where $\sigma$ is the surface tension, $\mathrm{R}$ is the bubble radius, $n_{g}$ the concentration of gas molecules and $\Delta \mu$ the difference in chemical potential between the gas and the liquid phase. At 
temperatures below the boiling point of the liquid, the chemical potential of the liquid phase is always higher than the gas phase $(\Delta \mu>0)$ and the liquid phase is the stable phase. At the boiling point the chemical potential of both phases is equal and $\Delta \mu=0$ and both phases can coexist. However, a gas bubble present in the liquid is always unstable and will immediately collapse, because a decrease in bubble diameter leads to a decrease in Gibbs energy. If the liquid is superheated beyond its boiling point $\Delta \mu<0$ and $\Delta G$ increases to a maximum at a certain radius and then decreases. The difference in Gibbs energy versus bubble radius is shown in figure 2.19. The maximum can be seen as barrier which must be surmounted at a significant rate before boiling will be observed. Newly formed bubbles with a radius smaller than the critical radius tend to collapse, and bubbles with a radius larger than the critical tend to grow spontaneously.

The critical radius $\left(R_{c}\right)$ and the height of the barrier $\left(\Delta G_{c}\right)$ ) can be calculated from 2.13 by taking $d \Delta G / d R=0$.

$$
\begin{aligned}
R_{c} & =\frac{2 \sigma}{n_{g} \Delta \mu} \\
\Delta G_{c} & =\frac{16 \pi \sigma^{3}}{3\left(n_{g} \Delta \mu\right)^{2}}
\end{aligned}
$$

The critical radius and barrier height are governed by the surface tension and the difference in chemical potential. An increase in temperature of a pure liquid leads to a decrease in surface tension and as already stated earlier an increase in $\Delta \mu$. Both factors lead to a lowering of the barrier to bubble nucleation and to an increase of the probability that bubbles surmount the barrier and grow spontaneously. The nucleation does not take place at any particular temperature, but can occur over a range of temperatures. The median temperature of this range is called the kinetic limit of superheat and unlike the thermodynamic limit of superheat this is not a true limit.

The chemical potential as well as the gas concentration are not easy to measure. It can however be assumed that the bubble is in or at least close to its mechanical equilibrium. In this case the Laplace equation 2.15 can be used to relate the radius to the pressure difference.

$$
P_{g}=P_{l}+\frac{2 \sigma}{R}
$$

where $P_{g}$ and $P_{l}$ are the pressures of the gas and liquid. The pressure inside the bubble will be the sum of the vapour pressure and the partial gas pressure of the gasses present in the bubble. The pressure of the vapour will be close to the saturation pressure for the temperature inside the bubble. The saturation pressure depends on the temperature and can be calculated using the Clausius-Clapeyron equation

$$
P_{\text {sat }}=P_{a m b} \exp \left[\frac{L}{k_{B}}\left(\frac{1}{T_{b}}-\frac{1}{T}\right)\right]
$$

where $P_{a m b}$ is the ambient pressure, $L$ is the latent heat of the liquid, $k_{B}$ is Bolztmann's constant and $T_{b}$ is the boiling temperature of the liquid at ambient pressure. The vapour pressure inside the bubble is different from the saturation pressure calculated using equation (2.16). 
The relation between the real pressure inside the bubble and the saturation pressure $P_{\text {sat }}$, also called the Poynting correction, is given by

$$
P_{g}=P_{\text {sat }} \exp \left[\frac{v_{l}\left(P_{l}-P_{\text {sat }}\right)}{R T_{0}}\right]
$$

where $T_{0}$ is the temperature of the liquid and the gas bubble and $v_{l}$ is specific volume of the the liquid. In the calculation it is assumed that the liquid is incompressible and that the whole system is at the same temperature $T_{0}$. In reality the liquid will be compressed near the bubble interface and a significant temperature difference will occur, especially if a liquid is heated to a high degree of superheat by short pulses. Equation 2.17 can thus only be used to give an indication of the pressure inside bubble. The critical bubble radius and the corresponding $\Delta G_{c}$ can also be expressed with respect to pressure and equation 2.14 will become

$$
\begin{aligned}
R_{c} & =\frac{2 \sigma}{P_{g}-P_{l}} \\
\Delta G_{c} & =\frac{16 \pi \sigma^{3}}{3\left(P_{g}-P_{l}\right)^{2}}
\end{aligned}
$$

The critical bubble radius and minimum Gibbs energy will both decrease with an increase in pressure difference between the liquid and the vapour inside the bubble. The pressure inside the bubble can be calculated from the temperature inside the bubble by using equation 2.16 and 2.17. The temperature in the bubble is assumed to be uniform and for a bubble situated on the heater, the bubble temperature will be nearly identical to the temperature of the heater surface. This temperature can be measured and from this the critical radius and minimum Gibbs energy can be estimated.

\subsubsection{Nucleation probability}

Under uniform conditions, for example in a pure liquid at uniform pressure and temperature, nucleation is a random process. The exact time and place of bubble nucleation cannot be predicted in advance. The nucleation probability, however, can be determined. Fluctuations in the density of the liquid can act as nuclei for bubble nucleation, this mechanism is called spontaneous nucleation. The size of these fluctuations depends on the local liquid temperature. The energy needed to create such a density difference is given by the Gibbs energy. If one considers a liquid with a number density $N$ the number of molecules with a energy $\Delta G$ at a temperature $T$ is given by

$$
n=N \exp -\frac{\Delta G}{k_{B} T}
$$

The value found for $n$ can also be seen as the number of nuclei with a difference in Gibbs energy of $\Delta G$. Only nuclei with a size near to the critical size and thus a energy $\Delta G_{c}$ can grow and become an actual bubble. Further derivation of the nucleation rate (probability) will not be given here and a more detailed explanation can be found in 
$[44,42]$. The nucleation rate (probability) according to the classical nucleation theory, per unit time and volume due to the thermal fluctuation of a liquid of temperature $T$ is

$$
J_{h o}(T)=N\left(\frac{3 \sigma}{\pi m}\right)^{1 / 2} \exp \left[-\frac{\Delta G_{c}}{k_{B} T}\right]
$$

where $m$ is the mass per molecules. This nucleation rate decribes the nucleation in the bulk of the liquid, also known as homogeneous nucleation. Therefore equation 2.20 is from now on called the homogeneous nucleation rate. The moment an interface or boundary is present, the mechanism of nucleation stays identical, but the amount of Gibbs energy needed to form a bubble with a critical radius will change. The amount of change depends on the contact angle $(\theta)$ of the liquid with the interface and on the geometry of the interface. The heterogeneous nucleation rate is also described by $[44,42]$ and is given by

$$
\begin{aligned}
J_{h e}(T, \theta) & =N^{2 / 3} \psi\left(\frac{3 \sigma}{\pi m \phi}\right)^{1 / 2} \exp \left[-\frac{\Delta G_{c} \phi}{k_{B} T}\right] \\
\phi & =\frac{2+3 \cos \theta-\cos ^{3} \theta}{4} \\
\psi & =\frac{1+\cos \theta}{2}
\end{aligned}
$$

For $\theta=0$ the liquid completely wets the surface and the heterogeneous nucleation rate becomes identical to the homogeneous nucleation rate, if $N^{2 / 3}$ is replaced by $N$. The difference occurs since one views each molecule at the surface instead of each molecule in the liquid as a potential bubble.

If a liquid is heated by pulse heating of a surface, the heterogeneous nucleation rate will determine the bubble nucleation rate at the heater surface. The liquid near to the surface will also be superheated and bubble nucleation can also take place in this part of the bulk liquid. The nucleation rate in the liquid will depend on the temperature distribution in the liquid. If this distribution only changes with the direction perpendicular to the heater surface, for example for the 1-D model discussed in 2.2, the nucleation rate in the liquid is given by

$$
K_{h o}=\int_{0}^{\infty} J_{h o}(z) d z
$$

The total nucleation rate $K$ per unit time will now be

$$
K=K_{h o}+J_{h e}
$$

The homogeneous as well as the heterogeneous nucleation rate increases quickly with temperature. A change of a few degrees in temperature can lead to a change of nucleation rate of several orders of magnitude. The heterogeneous nucleation rate, in contrast to the homogeneous nucleation rate, depends on the contact angle and local geometry. For low contact angles the homogeneous and heterogeneous nucleation rates are of the same order magnitude. At $\theta=0$ they differ only by the factor $N^{1 / 3}$. At a contact angle of approximately $68^{\circ}$, the two modes are equally probable. For higher contact angles the heterogeneous nucleation rate exceeds the homogeneous nucleation rate and will be the 
dominant nucleation mechanism. For hydrophilic surfaces nucleation will thus be mostly homogeneous and for hydrophobic surface there will be more heterogeneous nucleation.

Up to now only flat surfaces were considered, but in reality heaters will not be atomically flat. A local cavity or crack can have a large effect on bubble nucleation. For example,the Gibbs energy required to form a bubble in a small conical shaped cavity will be much lower than the energy needed to create a bubble with a similar curvature on a flat surface. The probability of nucleation at these sites will be much higher than the nucleation at the heater or in the liquid. These sites are thus preferred sites for bubble nucleation.

\subsubsection{Spontaneous nucleation}

The nucleation of bubbles, heterogeneous or homogeneous, due to thermal fluctuations is called spontaneous nucleation. This type of nucleation will only occur close to the limit of superheat. Fluctuations in the liquid density can grow to sizes of the critical radius. For most liquids and pressures close to the ambient pressure the temperature at which the limit is reached occurs is around $90 \%$ of the critical temperature. The model from section 2.2 can be used to predict the time of nucleation. Equation 2.8 is used to calculate the time it will take the heater to reach a nucleation temperature fixed at $90 \%$ of the critical temperature. Rewriting the equation shows the influence of the applied heat-flux on the nucleation.

$$
t=\frac{C}{q^{2}}
$$

Here $C$ is a constant, which is the combination of the thermal properties of the liquid and the substrate, and the nucleation temperature. Figure 2.20 shows the nucleation time as a function of applied heat-flux. In the calculations properties of ethanol for the liquid, pyrex for the substrate, a nucleation temperature of $205^{\circ} \mathrm{C}$ and a initial temperature of $25^{\circ} \mathrm{C}$ were used. The figure shows a quick drop in nucleation time as the applied heatflux increases. Of course this model is only correct if bubbles are formed by spontaneous nucleation. A defect or cavity will locally lower the energy barrier for bubble nucleation and the probability of nucleation at that site will increase. Furthermore nucleation can take place at lower temperatures and will thus take place earlier at these sites. It is also possible that a gas bubble gets trapped in a crack or crevice. These bubbles will also start to grow at the moment a certain energy threshold is crossed. This threshold depends on the local pressure and temperature. Often growth of these bubbles can take place at temperatures far below the spontaneous nucleation temperature.

There are two ways to ensure that spontaneous nucleation is the main nucleation mechanism. The first is to have no defects or cavities by using atomically flat heaters and extremely pure liquids. Also the device that holds the liquid has to be free from cavities or defects. The second option is to use high heat-fluxes. For low heat-fluxes the total nucleation rate is low and nucleation will only take place at the sites with the highest probability. Cavities and crack in the surface and trapped gas bubbles are such sites and nucleation and growth will mainly take place from these sites. As the heat-flux increases the nucleation rate will also increase and nucleation from sites other than preferential sites has to occur. This can happen on the heating surface due to heterogeneous nucleation or 


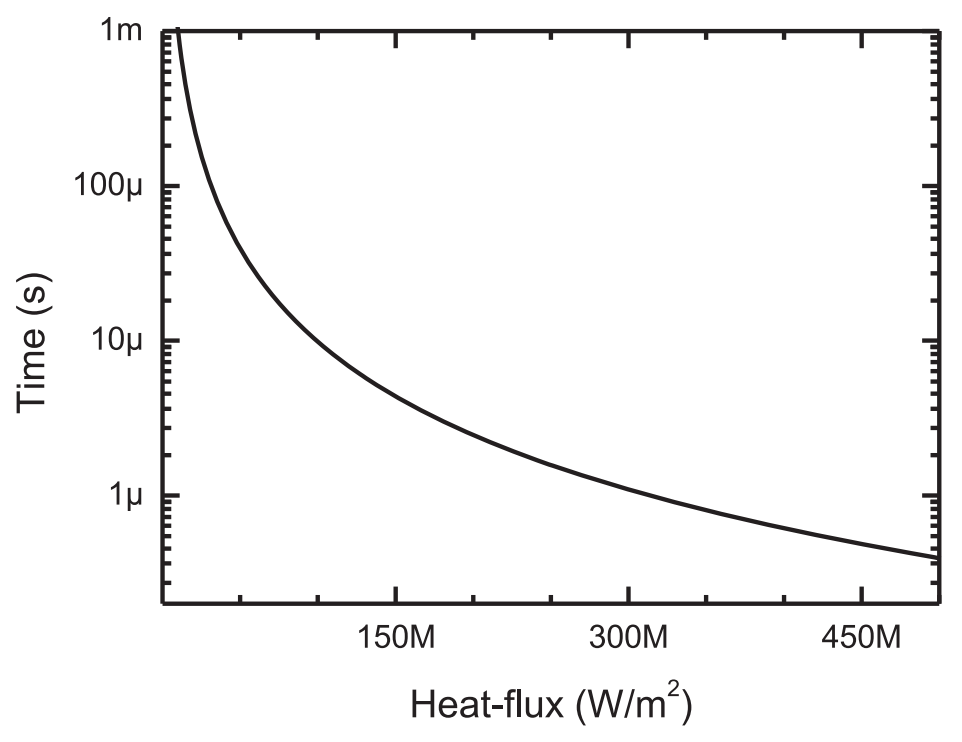

Figure 2.20: Time of bubble nucleation after start of the heating pulse as a function of the applied heat-flux. The 1-D conduction model, a nucleation temperature of $205^{\circ} \mathrm{C}$ and an ambient temperature of $25^{\circ} \mathrm{C}$ were used in the calculations

in the liquid due to homogeneous nucleation. At high heat-fluxes the number of bubbles that grow from the limited amount of preferential sites will be small in comparison to the number of bubbles formed by spontaneous nucleation in the liquid or at the heater surface. Furthermore the difference in nucleation times between the nucleation at preferred sites and spontaneous nucleation is small at high heat-fluxes. The increase, from ambient temperature to the temperature at which spontaneous nucleation can occur, takes place in a few microseconds. There will only be a difference of a few tenths of a microseconds between bubble nucleation at preferential sites and spontaneous nucleation.

\subsection{Bubble dynamics}

The dynamical behaviour of the bubbles is very complex. The rapid heating of the liquid leads to the introduction of large amount of energy in a short time and in a small volume. This can lead to a vapour explosion. The vapour volume will grow at a high rate and the kinetic energy from this expanding bubble will be utilized to deflect the membrane. The behaviour of the bubble with respect to time can be divided in several stages. In the first stage the bubble grows explosive due to a vapour pressure inside the bubble. As the bubble size increases the pressure inside the bubble will drop and the growth slows down. After this initial growth, periods of bubble growth and bubble collapse can alternate. This is typical for the second or intermediate stage of bubble dynamics. The third stage corresponds to the bubble collapse after the heating is stopped. 


\subsubsection{Explosive evaporation}

Spontaneous nucleation of a large number of bubbles in a highly superheated liquid can lead to explosive evaporation. The power unleashed by the transition from the liquid to the vapour phase will depend on the pressure inside and volume of the initial bubble(s). The initial bubble radius will be very small and can be estimated using equation 2.18. The initial pressure, which will be close to the vapour pressure $\left(P_{\text {sat }}\left(T_{v}\right)\right)$ at the nucleation temperature, will be much higher than the ambient pressure. For ethanol the initial bubble radius will be a few nanometers and the initial bubble pressure is estimated at several megapascal [56].

The growth is controlled by the pressure difference between the vapour inside the bubble and the liquid surrounding the bubble and the difference in condensation and evaporation rates at the bubble interface. Mikic et al. [81] divided the bubble growth in two regimes; the inertia controlled growth and the heat diffusion controlled growth. The bubble growth in the early stage before the cooling effect becomes significant is mainly determined by the liquid inertia. In this regime the speed of the bubble interface can be approximated by the asymptotic Rayleigh bubble growth rate:

$$
\frac{\partial R}{\partial t}=\sqrt{\frac{2\left[P_{s a t}\left(T_{v}\right)-P_{a m b}\right]}{3 \rho_{l}}}
$$

where $R$ is the radius of a nucleus and $\rho_{l}$ is the density of the liquid. In this case the bubble growth rate is independent of time and therefore the radius of the bubble will increase linearly with time. The bubble growth in a superheated liquid controlled by heat diffusion was described by Plesset and Zwick [41]. The growth speed is determined by the difference between evaporation and condensation at the bubble interface. In the heat diffusion controlled regime the bubble radius grows with the square root of time.

In the case of spontaneous nucleation a number of bubbles nucleate at the almost the same time. The bubbles will quickly grow and coalesce forming a thin vapour film before they grow large. Furthermore the duration of the heat pulse is short and the superheated liquid layer is so thin that the temperature and pressure of the bubble rapidly decrease before the vapour film grows large. In such a highly subcooled condition, the liquid is given an initial supply of kinetic energy by the pressure impulse. The bubble growth is dynamically controlled by the pressure difference and liquid inertia.

Asai et al. [56] proposed the following theoretical model for the bubble volume generated by a pressure impulse. In the bubble growth phase, the bubble motion was approximated by a one-dimensional growth of a vapour film. The pressure inside the bubble is given by

$$
P_{v}(t)=\left[P_{n u c}-P_{s a t}\left(T_{a m b}\right)\right] \exp \left[-\left(\frac{t}{t_{e}}\right)^{\lambda}\right]+P_{\text {sat }}\left(T_{a m b}\right)
$$

where $P_{n u c}$ is the pressure inside the bubble at nucleation, $P_{\text {sat }}$ is the saturation pressure, $T_{a m b}$ is the ambient temperature, $t_{e}$ is a time constant, which depends on the heating condition and thermal property of the working fluid and $\lambda$ is a free parameter with a value between 0.5 and 1.5. The values of $t_{e}$ and $\lambda$ must be determined from experimental results. 
The bubble volume can be calculated by the following equations:

$$
V_{v}(T)=\frac{W\left[2 \xi-\xi^{2}\right]}{P_{a m b}-P_{s a t}\left(T_{a m b}\right)}
$$

where

$$
\xi=\frac{\left[P_{a m b}-P_{s a t}\left(T_{a m b}\right)\right] t}{M_{p}}
$$

The pressure impulse, $M_{p}$, and the work done by the bubble to the liquid, $W$, are given by

$$
\begin{gathered}
M_{p} \approx \frac{P_{n u c} t_{e}}{\lambda} \\
W \approx \frac{M_{p}^{2}}{2 A_{l}}
\end{gathered}
$$

where $A_{l}$ is the inertance of the liquid region. The value of $A_{l}$ strongly depends on the shape of the liquid region. For a heater with square sides of $d_{h}$ located on a flat wall and a liquid with density $\rho_{l}$, which fills a semi-infinite region defined by the wall and an infinite open boundary, the inertance is given by

$$
A_{l} \approx 0.43 \frac{\rho_{l}}{d_{h}}
$$

The bubble volume is highly dependent on the initial pressure impulse, the ambient pressure and the ambient temperature and it scales linear with the heater size. The pressure impulse is related to $t_{e}$ and $P_{n u c}$, both values depend on the heating conditions. $P_{n u c}$ can be estimated using equations 2.16 and 2.17. The relation between $t_{e}$ and the heating conditions is more complex. A further discussion on $t_{e}$ can be found in the paper written by Asai et al. [56].

In section 2.2.2 the temperature distribution in the liquid was calculated. For a short heating pulse a small area of liquid is heated and the growth can be described by the theory described above. The energy introduced before the nucleation will determine the pressure impulse and therefore the maximum volume of the vapour sheet. For longer pulses the heat penetration depth is much larger and the profile becomes more rounded. The bubble growth will not only be influenced by the initial pressure pulse, but also by the temperature difference across the bubble interface. The bubble volume will be larger and more rounded due to the surface tension and temperature distribution. It will depend on the heating conditions, such as pulse time and power, when the bubble growth will stop and the collapse starts. 


\subsubsection{Bubble collapse}

After the maximum bubble volume is reached the bubble will start to collapse. In a highly subcooled liquid the bubble can be treated as a cavitation bubble. The collapse of such a bubble was described by Rayleigh [82] and the collapse time for a vapour is given by

$$
t_{c o l}=0.915 R_{\max } \sqrt{\frac{\rho_{l}}{P_{a m b}-P_{s a t}\left(T_{a m b}\right)}}
$$

where $R_{\max }$ is the maximum bubble radius. In subcooled liquid the pressure difference between the liquid pressure, $P_{a m b}$ and the vapour pressure at ambient pressure, $P_{s a t}\left(T_{a m b}\right)$, will be large. The bubble quickly collapses and the vapour will condensate.

If, however, the bubble not only contains vapour but also other gasses, the bubble collapse will be much slower. The gas has to diffuse into the liquid and this will take much longer. Initially the concentration of gasses in the vapour will be equal to the concentration in the liquid. After the maximum bubble size is reached the bubble quickly decreases in size. The vapour will start to condensate and the gas concentration in the bubble increases. The concentration gradient across the bubble interface and the diffusion speed of the particular gasses through the liquid will determine the collapse speed of the gas bubble.

\subsubsection{The intermediate stage}

In most cases the bubble will not collapse completely in its first collapse period. The bubble collapse will induce a flow of cold liquid to the heater. If the heater is still above the nucleation temperature fresh vapour is produced and the bubble can grow again. If the heating is continued during this intermediate phase the alternating growth and collapse will continue. The difference in maximum and minimum bubble size will decrease and the time between periods of growth and collapse will increase. This will result in a permanently growing bubble or a stable bubble depending on the heating power. The difference between the maximum and minimum bubble volume and the time of the growth and collapse periods depend on the heating conditions and the heater size. The bubble dynamics during this stage are very complex and will have a large effect on the temperature distribution of the liquid. Besides a relatively simple growth and collapse, jets can be formed or fragmentation of the bubble into smaller droplets can occur.

\subsubsection{Summary and conclusions on the bubble dynamics}

The spontaneous nucleation process, is a random event, but because the nucleation probability increases quickly with temperature, the nucleation time, temperature and location can be controlled with a high accuracy at high heating rates (more than $6 \times 10^{6} \mathrm{~K} / \mathrm{s}$ ). In these cases the temperature of nucleation will be about $90 \%$ of the critical temperature of the liquid. The time of nucleation depends on the heat-flux and the properties of liquid and substrate, such as thermal conductivity, diffusivity and nucleation temperature. The heat-flux determines the speed of temperature increase for a given liquid and substrate and thus the time at which the nucleation temperature of the liquid is reached. At high 
heat-fluxes the time of nucleation can therefore be controlled with a precision of less then a microsecond.

As mentioned earlier bubble nucleation is a random process. At first an initial bubble nucleus with a radius larger than the critical radius must be present or created. Nuclei with a radius smaller than the critical radius will simply collapse. These nuclei can already be present in the liquid or at the surface in the form of trapped gas bubbles. They can also be generated by spontaneous nucleation, where fluctuations in the liquid density act as bubble nuclei. Particles in the liquid and cavities and cracks at the surface are preferential sites for bubble nucleation. The nucleation rate (probability) increases extremely fast with temperature. A quick rise in temperature will therefore result in the creation of a large number of nuclei in limited amount of time all over the superheated region. Although bubble nucleation at the preferred sites will take place at a lower temperature and thus earlier, the fast rise in temperature limits difference in nucleation time. The number of bubbles created at preferential sites is also relatively low at these high heat-fluxes and their influence is therefore reduced.

During spontaneous nucleation a large number of small bubbles will be formed across the heater. The initial pressure inside these bubbles will be several megapascal and they will grow explosively. The bubbles will coalesce and form a vapour sheet covering the heater. The pressure and temperature inside the vapour sheet will quickly decrease after the sheet is formed. The growth will be dynamically controlled and determined by the pressure impulse generated at the moment of nucleation and the liquid inertia. After the maximum bubble volume is reached, bubble cavitation will occur. The speed of collapse of the vapour bubble will depend on the pressure difference between the liquid and the vapour pressure at ambient temperature.

Three stages can be distinguished; the growth stage where the bubble is formed by explosive evaporation and the bubble quickly grows, the intermediate stage with alternating periods of growth and collapse and the final stage in which the vapour condensates and the system return to its initial state. The length and the power of the heating pulse will determine if the bubble will directly collapse or go through the intermediate stage. If a small amount of gas is dissolved in the liquid, the bubble will also contain some gas; after the vapour has condensed a bubble containing only gas will remain. This bubble has to dissolve in the liquid and this will take much more time than condensation.

\subsection{Membrane deflection theory}

A membrane can be seen as a thin diaphragm clamped rigidly at the edges. In most cases one speaks of a membrane if the thickness is small in comparison to the radius of the diaphragm. A membrane can be considered as the two-dimensional counterpart of a stretched wire or string. A force on the membrane will result in a membrane deflection. The amount of the deflection will depend on the tensile resistance of the diaphragm. A pure membrane is not able to support shear forces, but in reality these forces give rise to elastic bending of the diaphragm. The bending resistance of a diaphragm depends on the ratio between the thickness and size of the diaphragm, it increases with diaphragm thickness. As the thickness of the membrane increases, the bending resistance becomes 
more important and the diaphragm has to be treated as flat plate rigidly clamped at the edges.

\subsubsection{Static deflection}

If several parameters, such as diaphragm thickness, size, Young's modulus and Poisson's ratio are known, one can calculate the displacement of a diaphragm under a constant pressure. Here only a short description of the theory on the calculation will be given. A more detailed description can be found in $[83,84,85,86]$. The relation between pressure and membrane deflection can be described by the characteristic cubic equation:

$$
P=c y+d y^{3}
$$

where

$$
\begin{aligned}
& c=c_{b}+c_{i}=\frac{16}{3\left(1-\mu^{2}\right)} \frac{E h^{3}}{a^{4}}+\frac{4 h \sigma_{0}}{a^{2}} \\
& d=\frac{7-\mu}{3(1-\mu)} \frac{E h}{a^{4}}
\end{aligned}
$$

$y$ is the deflection at the centre, $\mu$ is the Poission's ratio, $E$ is the Young's modulus, $\sigma_{0}$ is the initial stress in the membrane, $P$ is the pressure difference, $a$ is the radius and $h$ is the thickness of the membrane. There are two regimes of deflection. The linear regime and the cubic regime. In the linear regime the deflection is small in comparison to the thickness of the membrane and the membrane mainly bends. The relation between deflection and pressure is linear and determined primarily by $c$. The linear coefficient (c) can be divided in two parts. $c_{b}$ is the influence of the bending energy and $c_{i}$ is the contribution of the initial or residual stress. For large displacements, the cubic regime applies, the deflection scales with the cubic root of the pressure, mainly due to tension in the membrane. The relation is determined mainly by $d$.

\subsubsection{Membrane vibrations}

Besides a static deflection the membrane can also have a varying displacement in time, which from now on will be called the dynamic deflection or displacement of the membrane. The dynamic deflection is the result of vibrations in the membrane. If a statically loaded elastic system is disturbed in some manner from its equilibrium, the internal forces and moments in the deformed configuration will no longer be in balance with the external loads and vibration will occur. The size, frequency and number of vibrations before the vibration has dampened out will depend on the mass, the spring stiffness and the damping of the system. In general the vibrations of an elastic system will occur at its resonance or natural frequencies. The number of natural frequencies is equal the total number of degrees of freedom. A diaphragm or membrane can be seen as a continuously distributed mass and elasticity. It therefore has an infinite number of natural frequencies. The pattern of the membrane deflection at a certain natural frequency is called a mode-shape. For several geometries the different modes of vibration and the corresponding frequency can be calculated analytically. 
The natural frequencies of a circular membrane for example are given by:

$$
f_{s, n}=\frac{\alpha_{s, n}}{2 \pi a} \sqrt{\frac{T}{\rho h}}
$$

here $T$ is the tension on the membrane, $\rho$ is the density of the membrane and $\alpha_{s, n}$ is a constant for a given number $n$ of nodal diameters (straight lines across the surface of membrane through its centre that do not deflect at that particular frequency) and a given number $s$ of nodal circles (circles on the membrane that show no deflection for that particular frequency).
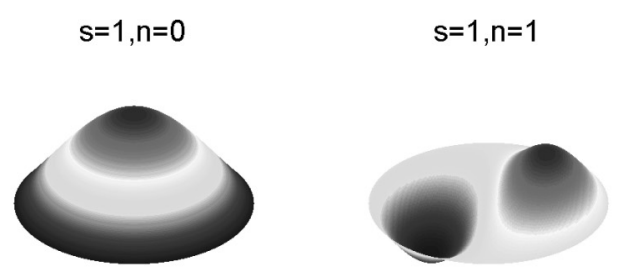

$$
\mathrm{s}=1, \mathrm{n}=2
$$
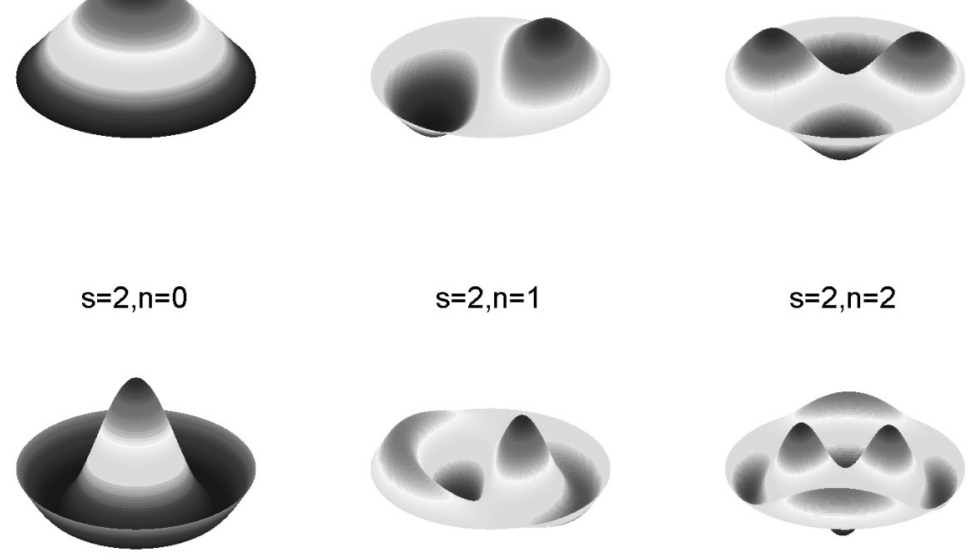

$$
\mathrm{s}=2, \mathrm{n}=2
$$

$\mathrm{s}=3, \mathrm{n}=0$

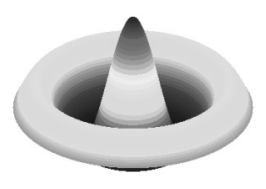

$s=3, n=1$

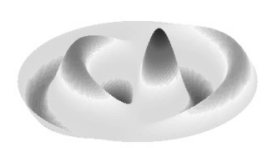

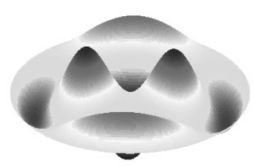

$\mathrm{s}=3, \mathrm{n}=2$

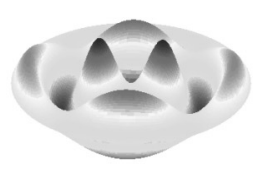

Figure 2.21: Mode-shapes for a circular diaphragm. The value of $s$ corresponds to the number of nodal circles and the value of $n$ corresponds to the number of nodal diameters.

A few values for $\alpha_{s, n}$ are shown in table 2.2 and figure 2.21 shows the corresponding mode shapes. The first nodal circle, $\mathrm{s}=1$, is the circle around the edge of membrane and the first nodal line, $\mathrm{n}=1$, is vertical line which will form if one connects the points across the membrane which do not deflect in the $\mathrm{s}=1, \mathrm{n}=1$ mode shape. 


\begin{tabular}{|c|c|c|c|}
\hline $\mathrm{s}$ & $n=0$ & $n=1$ & $n=2$ \\
\hline 1 & 2.404 & 3.832 & 5.135 \\
2 & 5.520 & 7.016 & 8.417 \\
3 & 8.654 & 10.17 & 11.62 \\
\hline
\end{tabular}

Table 2.2: Values for $\alpha_{s, n}$ for a circular membrane

As with the static deflection there are two regimes. The diaphragm can be treated as a pure membrane, where restoring force comes from the membrane tension and as a plate where the restoring force is due to the structural stiffness. For a plate clamped at the edges the mode-shapes stay the same. The equation for the natural frequencies however is slightly different:

$$
f_{s, n}=\frac{\alpha_{s, n}}{2 \pi a^{2}} \sqrt{\frac{D}{\rho h}}
$$

where $D$ is the flexural rigidity and given by

$$
D=\frac{E h^{3}}{12\left(1-\mu^{2}\right)}
$$

The values for $\alpha_{s, n}$ are also slightly different and shown in table 2.3. The main difference

\begin{tabular}{|c|c|c|c|}
\hline $\mathrm{s}$ & $n=0$ & $n=1$ & $n=0$ \\
\hline 1 & 10.21 & 21.26 & 34.88 \\
2 & 39.77 & 60.82 & 84.58 \\
3 & 89.11 & 120.1 & 153.8 \\
\hline
\end{tabular}

Table 2.3: Values for $\alpha_{s, n}$ for a circular clamped plate

between the two regimes is that for the membrane the frequency scales with inverse of the radius of the diaphragm and for the plate it scales with the inverse of the square of the radius. Furthermore the relative differences between the coefficients changes.

An analytical solution for modes of vibration and the corresponding frequencies for a square membrane also exists. The natural frequency is given by

$$
f_{m, n}=\frac{1}{2 a} \sqrt{\frac{T}{\rho h}\left(m^{2}+n^{2}\right)}
$$

where $\mathrm{n}$ and $\mathrm{m}$ are the nodal lines. Figure 2.22 shows the mode-shapes for the square membrane. Because all the edges of the membrane are equal, two or more mode-shapes can correspond to a single frequency. The mode with $m=2$ and $n=1$ has the same natural frequency as the $\mathrm{m}=1$ and $\mathrm{n}=2$ mode. In this case both mode-shapes can coexist and actual mode-shape will be a superposition of the two. $A$ and $B$ are the weights of the two modes. The extreme cases, $A=0, A=B$ and $A=-B$, are shown in figure 2.22. For all other values the interior nodal line is curved. For the clamped square plate there is no analytical solution and the natural frequencies have to be found by approximations or numerical calculations. 


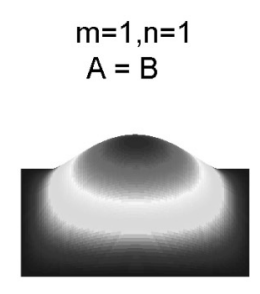

$$
\begin{gathered}
m=2, n=1 \\
B=0
\end{gathered}
$$

$\mathrm{m}=2, \mathrm{n}=1$

$A=B$
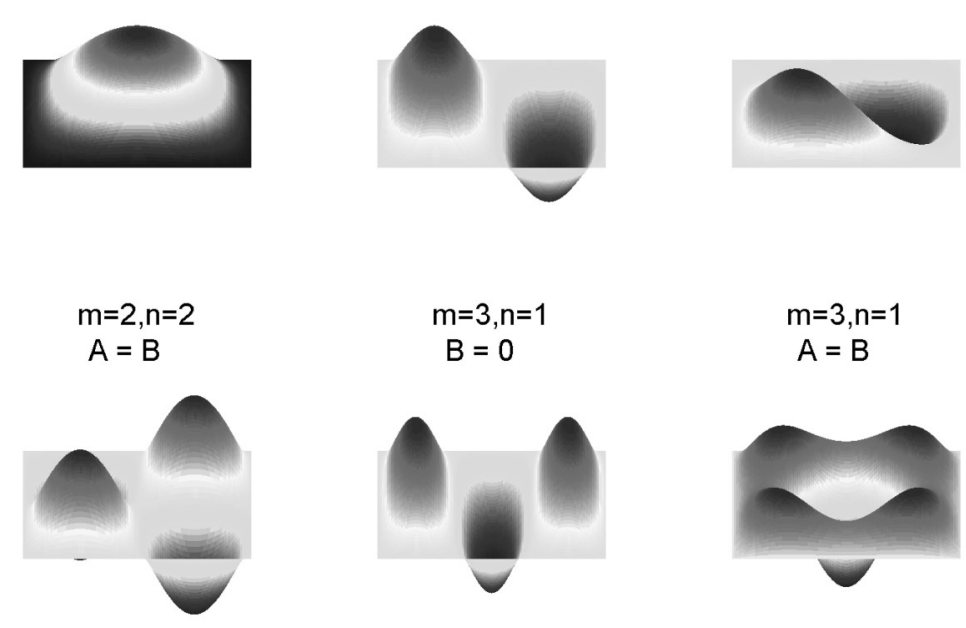

$$
\begin{gathered}
m=3, n=1 \\
A=-B
\end{gathered}
$$

$$
\begin{gathered}
m=3, n=1 \\
B=0
\end{gathered}
$$

$m=3, n=1$

$A=B$
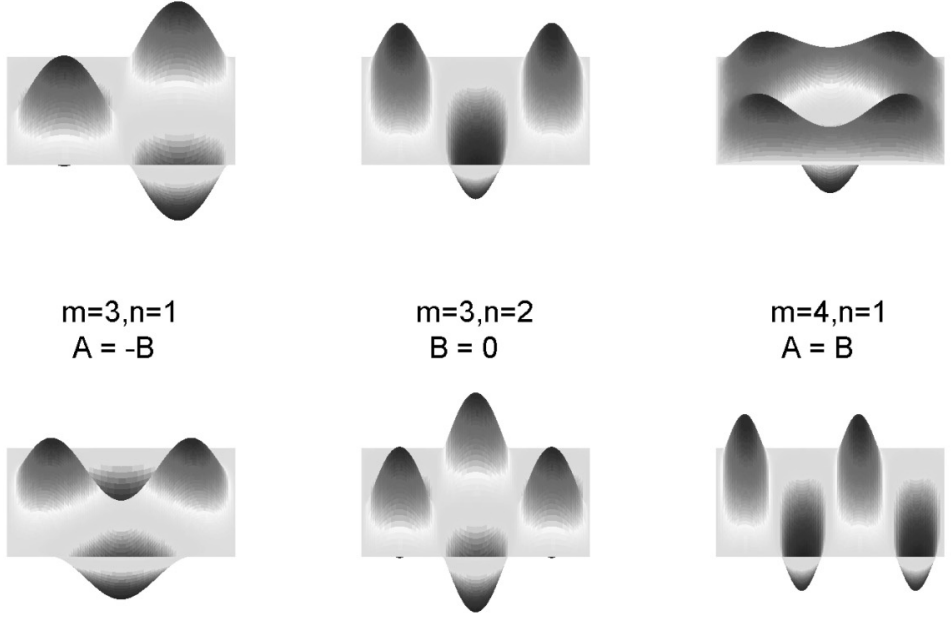

$$
m=4, n=1
$$$$
A=B
$$
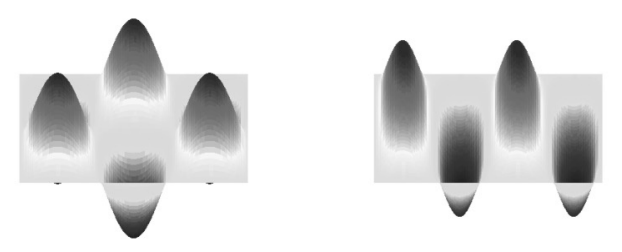

Figure 2.22: Mode-shapes for a square membrane. The value of $m$ and $n$ correspond to the number of nodal lines. 
The presence of a medium in contact with the diaphragm has two effects. Firstly, the frequency is lowered on account of the increase of effective inertia of the diaphragm due to the added mass of the medium vibrating in phase with the diaphragm. Secondly the vibrations are damped owing to an increased viscous friction and acoustic radiation. Lamb [87] has found, in the case of a diaphragm with one side in contact with water, that the inertia of the diaphragm is increased by the factor $(1+\beta)$ where

$$
\beta=0.67 \frac{\rho_{1} a}{\rho h}
$$

where $\rho_{1}$ is the density of water. The fundamental frequency $\left(f_{l}\right)$ in this case therefore becomes

$$
f_{l}=\frac{f_{n}}{\sqrt{1+\beta}}
$$

here $f_{n}$ is the fundamental frequency without the liquid. The effect of water is not identical for all natural frequencies, for the higher natural frequencies the value of 0.67 in equation 2.39 is different. Kwak $[88,89]$ calls this factor the nondimensionalized added virtual mass incremental factor(NAVMI). This factor is a function of vibrational modes and boundary conditions. The NAVMI factor and thus the effect of the added mass decreases as the number of nodal lines and nodal circles increases. The fundamental mode is thus effected the most by the presence of water.

Identical to a simple mass and spring-system a diaphragm can be in free-vibration, but because instead of one degree of freedom it has an infinite number of degrees of freedom, the free-vibration will be a superposition of all the mode-shapes, each at its own natural frequency. If a membrane is exited for example by a single stroke it will start to vibrate at a large number of frequencies. The size and speed of the oscillations on each point of the membrane will not only depend on the diaphragm parameters, but also on the force and place of impact of the stroke on the diaphragm. In particular the initial deflection can be seen as a superposition of all initial states of the mode-shapes. The moment the diaphragm is released, it starts to vibrate. The total vibration is a superposition of all the mode-shapes oscillating with their own natural frequency. If there is no damping the diaphragm would vibrate endlessly and the amplitude of each mode-shape would be the same as in the initial state. Normally however, there is damping and the vibration decreases with time. The degree of persistence of the vibrations is indicated by the number of vibrations which elapse while the amplitude diminishes by the ratio $1 / \mathrm{e}$. The persistence depends on a large number of system parameters and will also be different for different mode-shapes [87]. The fundamental mode-shape will be highly dampened, because a net volume displacement takes place. During this volume change compression and expansion of the liquid takes place. This will result in fast dissipation of energy due to friction and a relatively small persistence. The first mode with a nodal line has a much higher persistence, because this mode requires no net change of volume. The liquid is moved from one side of the nodal line to the other. The time of decrease of certain mode will not only depend on the persistence of that particular mode, but also on its frequency. At high frequency it simply takes less time to preform a fixed number of vibrations. The amplitude of the mode-shapes will thus change in time, depending on persistence and the frequency of the particular mode. 
In the theory described above, the influence of the size and geometry of the cavity and filling channels were neglected. It, however, is possible to create standing wave patterns inside the cavity. In this case the lowest frequency $f$ of these standing waves will be close to

$$
f=\frac{c}{2 l}
$$

where $c$ is equal to the speed of sound in the local medium and $l$ is the size of cavity in a certain direction. If the frequencies expected from the membrane vibrations are comparable to the frequencies expected from the cavity resonance the two cannot be treated separately and a coupling between the membrane and cavity will occur. This will increase the complexity of the system even further. The dimensions of the cavity and the membrane must be chosen in such a way that the resonance frequencies are well separated. In the same manner there can be a coupling between pressure waves travelling through filling channel and the rest of the system. This can be prevented by using long channels with a high hydrolic resistance, because this will diminish the liquid flow and highly dampen pressure waves.

\subsubsection{Summary and conclusions on the membrane response}

The membrane displacement can be divided in a static and a dynamic deflection. The static deflection is generated by a constant pressure difference across the membrane. For small deflections in comparison to the membrane thickness the bending is dominant and the relation between displacement and pressure is mostly linear. For large deflection the tension in the membrane is dominating and in this regime the relation is cubic. A large initial stress has an influence mainly on the linear regime.

A dynamic deflection of the membrane is generated by a sudden change of pressure difference across the membrane. In this case a single pressure pulse is considered. After an initial deflection the diaphragm will start a free-vibration. The initial deflection and freevibration will be a superposition of all the mode-shapes of the diaphragm and each modeshape will oscillate at its particular natural frequency. The distribution of the mode-shape depends on the initial deflection generated by the bubbles. The natural frequencies of the membrane can be calculated. For an ideal membrane the natural frequency changes with applied membrane tension and scales with the inverse of the radius. For a plate clamped at the edges the natural frequency depends on the flexural rigidity and scales with the inverse square of the radius.

The presence of a different medium on one side of the membrane leads to a change in natural frequency. The frequency is lowered due to the added mass of the medium and the vibrations are dampened due to an increased viscous friction and acoustic radiation. The influence on each mode is different, but in general the fundamental mode is affected most.

Mode-shapes, which require a net volume displacement, are highly effected by damping and will have a low persistence. The distribution of the individual mode-shapes will thus change in time. The response of the membrane after a single pressure pulse generated by a bubble will thus depend on a large number of parameters. The initial pressure impulse with the membrane properties, such a size, thickness, Young's modulus and initial 
stress, will have a large influence on the initial displacement. In order to generate a large deflection the membrane should be relatively flexible. The behaviour afterwards will again be determined by the membrane properties, but here also the properties of the liquid on one side of the membrane will be important. After the initial deflection the membrane should quickly return to its original state without a large number of vibrations. A stiff membrane and a large damping would in this case be preferable. A good consideration of the design and the materials should make it possible to achieve both requirements. 


\section{Chapter 3}

\section{Design and Fabrication}

The explosive micro-bubble actuator is fabricated using MEMS technology. The device contains a heater, a cavity filled with liquid and a membrane. Each part of the device has its own design issues and specific fabrication processes. At the end of the chapter the setup used for the measurements will be discussed. 


\subsection{Introduction}

The explosive micro-bubble actuator is fabricated using MEMS technology. This technology enables the fabrication of micrometer sized structures using semiconductor fabrication technology. The actuator can be divided in two main parts, shown in figure 3.1. The first

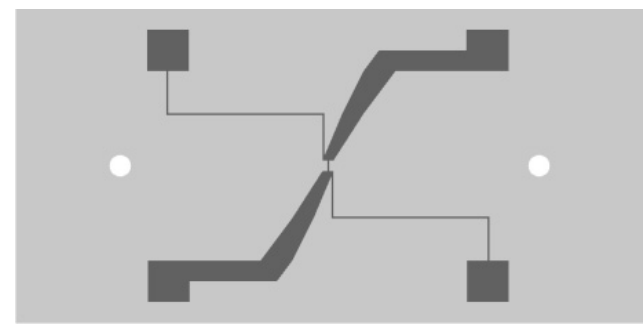

(a) Pyrex part

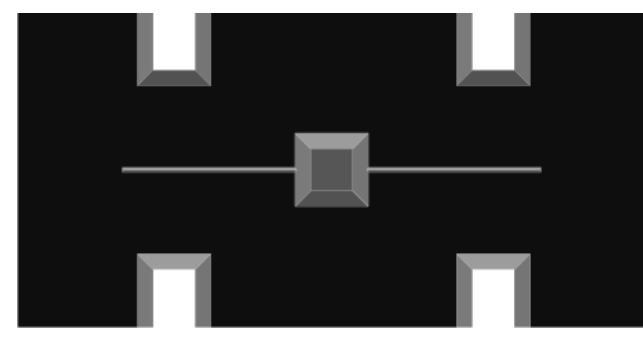

(b) Silicon part

Figure 3.1: Drawing of the two separate parts of the explosive Micro-bubble actuator.

part contains the heaters and leads and filling holes. The second part contains the membrane, a cavity, channels and holes to make electrical contact to the leads on the pyrex part. The design of the actuator focussed on two aspects; the heaters and the membrane. A thin metal film can dissipate a large quantity of energy in a short period and the resistance of the film changes with temperature. The metal film therefore is used as a heater and as well as a temperature sensor. Several different geometries and sizes of heaters and different types of leads are fabricated by thin film deposition and lift-off. Two different techniques were used to remove the Silicon underneath a thin Silicon-Nitride layer in order to form a membrane. In the first a $\mathrm{KOH}$ solution is used to remove the silicon by wet-etching. The second method uses a reactive ion etcher to remove the silicon by dry-etching. Square membranes were fabricated using $\mathrm{KOH}$ etching and circular membranes were fabricated using RIE. In the final step the two parts of the actuator were combined by anodic bonding to form the micro-bubble actuator. The experimental setups are discussed in the last part of the chapter. Two different types of measurements are conducted; the bubble are imaged by stroboscopic imaging and the deflection of the membrane is measurement by a laservibrometer.

\subsection{Design and fabrication of the heaters}

One of the most important parts of the micro-bubble actuator is the heater. The heaters should heat the liquid to a temperature of a several hundred degrees Celsius within a few micro-seconds. From the theory in the previous chapter it was calculated that this corresponds to a dissipation of hundreds of $\mathrm{MW} / \mathrm{m}^{2}$. The total power consumption can be limited by using micro-meter sized heaters. In this case a power in the order of one Watt is needed to create a bubble and the total energy dissipated per pulse is several micro Joules. In MEMS fabrication a large number of materials are used to fabricate heaters. In the majority of the cases metals like platinum [90, 49, 71, 74, 68], gold [20, 10, 91], aluminium $[92,93]$ or other pure metals are used as the heater material. However, not 
only pure metals, but also alloys and mixtures of materials can be used. Avedisian et al. [60] uses a mixture of Tantalum and Aluminum, the thermal inkjet head used in [23] and [55] has a heater of Hafnium-Bromide. Transparent heaters can be made from indiumtin-oxide [6]. It also possible to make a non-metallic heater from poly-silicon $[29,61,70]$ and Gluche et al. [94] uses a diamond film as a heater. Most of these materials can be deposited by thin film deposition. There are two different techniques for thin film deposition; sputtering and evaporation. The difference between the techniques is the directionality of the deposition. Evaporation occurs under much lower pressures and can therefore be deposited one-directional, where sputtering is always multi-directional.

The heaters should have patterns in the micrometer range. There are a few methods to create such patterns in a metal layer. One can use a shadow mask during metal deposition or create patterns in a metal film by selectively removing the metal by etching in a designated chemical solution. For the fabrication of the heaters and leads of the micro-bubble actuator lift-off is used to create a metal pattern. First a pattern is created in a layer of photo-resist by optical lithography. In a second step metal is deposited either by sputtering or evaporation. Parts of the substrate are now covered by a layer of resist with a metal layer on top and parts only have a metal layer. In the next step the resist is removed, leaving only a metal layer at the parts which were not covered by the resist. The resolution is not only determined by the lithography, but also by the difference in layer thickness between the resist and metal and by the profile of the resist. A relatively small difference in layer thickness, especially for thick metal layers, can result in a relatively rough edge, because the metal layer really has to break. In order to improve the sharpness of the edges of the pattern a image-reversal resist in combination with evaporation can be used. Because the edge of the image-reversal resist are negatively tapered they can act as a sort of local shadow mask if one-directional deposition by evaporation is used.

Platinum is used as the heater material for the micro-bubble actuator. This metal was chosen for the following reasons:

- Platinum is highly inert. The chemical inertness of a material is an indication how reactive a material is. If a liquid or a gas is subjected to an electrical conductor it is possible to get an electrochemical reaction and under some conditions this leads to deterioration and even destruction of the heater. It will depend on the conductor materials as well as on the medium to which the conductors are exposed, if such a reaction can take place. Water for example can be changed in to hydrogen and oxygen if two metal wires with a potential difference in between them are inserted in the liquid and iron subjected to moist air can lead to corrosion. A platinum heater does not react with the working liquid and therefore does not need an extra protective layer to prevent chemical reactions with the working liquid.

- The resistivity of platinum is low. This reduces the potential difference needed to heat the metal.

- Platinum has a relatively high temperature coefficient and up to about $800^{\circ} \mathrm{C}$ the temperature coefficient is also linear. This makes it possible to do resistance thermometry [95] and use the heater also as a temperature sensor. 
Unfortunately a noble metal like platinum does not adhere well to dielectric layers like silicon-dioxide and silicon-nitride. Platinum does not form a chemical bond with the atoms of the surface layer of the dielectric material. More reactive metals can be used as intermediate layer between the dielectric and the platinum film to promote adhesion. Metals commonly used for adhesion purposes are aluminium, chromium, titanium, tantalum and zirconium. For high temperature applications in micro-mechanics tantalum was found to be the best metal [96] . Up to $600^{\circ} \mathrm{C}$ it should be physically, electrically and chemically stable, at higher temperatures degradation of the layer or inter-layer diffusion can occur.

\subsubsection{Design}

The heaters are patterned using optical lithography, which with the equipment available in the lab results in a pattern resolution of $2 \mu \mathrm{m}$. This provides a large freedom in the design of the heaters. Still there are a few limitations one has to keep in mind. The heat-fluxes of more than $100 \mathrm{MW} / \mathrm{m}^{2}$, which are needed to generate the bubbles, in combination with the relatively thin metal layers lead to high current densities through the heater. It is known that at extremely high current densities electro-migration can lead to the damage of a metal layer. Unfortunately no investigations concerning electro-migration in a thin platinum film could be found in the literature. For other metals like copper and aluminium maximum current densities between $1 \times 10^{10} \mathrm{~A} / \mathrm{m}^{2}$ and $1 \times 10^{12} \mathrm{~A} / \mathrm{m}^{2}$ were found [97]. The limiting current density for platinum is expected to be in the same regime. The exact value can depend on a large number of parameters, such as deposition conditions, heater design and substrate material.

The maximum surface area of the heater is not limited, however, as the surface area increases more power is needed create the necessary heat-flux. In order to limit the power consumption to a few Watt a maximum heater area of $250 \mu \mathrm{m}$ x $250 \mu \mathrm{m}$ was used to place the heaters. These dimensions were also chosen, because such an area is relatively easy to view with a microscope.

A large number of different heater structures were fabricated. Several parameters were considered in the design. The resistance of the heater $\left(R_{\text {heater }}\right)$ is given by

$$
R_{\text {heater }}=\rho \frac{l}{w h}
$$

where $\rho$ is the resistivity of the platinum, $l$ is the total length of the heater, $w$ is the width of the heater and $h$ is the thickness of the heater. The resistivity of platinum is $1.05 \times 10^{-7} \Omega \mathrm{m}$ and the aimed layer thickness is between $100 \mathrm{~nm}$ and $200 \mathrm{~nm}$. The resistance of the heater is thus mainly determined by the ratio between the length and the width. Energy will be dissipated when a potential difference $V$ is applied across the heater. In this case the current density becomes

$$
j=\frac{I}{A}=\frac{V}{R_{\text {heater }} w h}=\frac{V}{\rho l}
$$

the heat-flux from the heater is given by

$$
q=\frac{P}{A_{\text {heater }}}=\frac{V^{2}}{R_{\text {heater }} A_{\text {heater }}}=\frac{V^{2} h}{\rho l^{2}}
$$


A minimum heat flux is needed to get spontaneous nucleation. A heater with a high resistance will need a high potential difference across the heater to reach this minimum heat-flux. Generating and measuring microsecond pulses of a high potential difference with a high accuracy requires specialized electronics. On the other hand a heater with a low resistance requires a relatively high current to generated the necessary heat-flux. If the current density gets to high this will lead to electro-migration and the heater will be damaged.

The bubble volume is expected to depend directly on the heater surface area. A larger surface area will simply lead to a larger bubble volume.

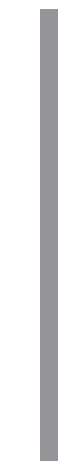

(a)

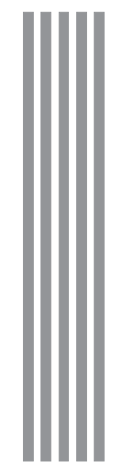

(b)

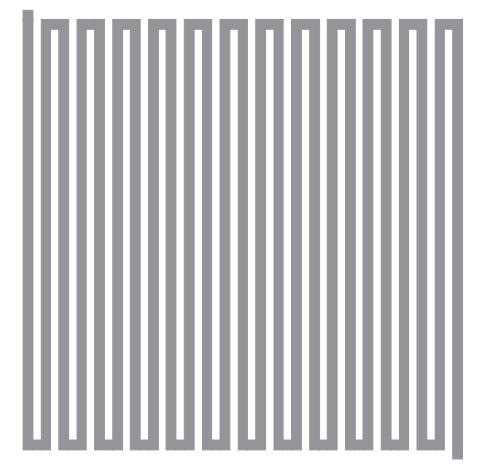

(c)

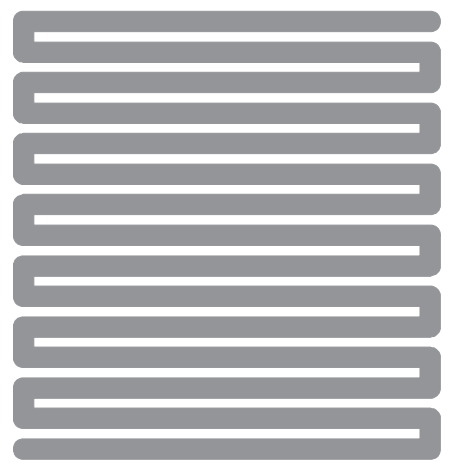

(d)

Figure 3.2: Different heater layouts; (a) a $10 \mu \mathrm{m}$ wide and $250 \mu \mathrm{m}$ long line, (b) five $5 \mu \mathrm{m}$ lines in parallel, (c)a vertical meander structure of $250 \mu \mathrm{m} \times 250 \mu \mathrm{m}$ with a line width of $5 \mu \mathrm{m},(\mathrm{d})$ a horizontal meander structure of $250 \mu \mathrm{m} \times 250 \mu \mathrm{m}$ with a line width of $10 \mu \mathrm{m}$.

The simplest structures are just straight lines and sheets. They were fabricated with widths between $2 \mu \mathrm{m}$ and $100 \mu \mathrm{m}$ (figure 3.2a). Especially the smallest lines have a rather large resistance and high voltages are needed to get the necessary heat-flux. The lines with the highest width have a relatively low resistance and one has to be careful not to exceed the maximum current density.

In order to decrease the resistance and increase the total surface area, while having the same line width, several of them are placed in parallel (figure 3.2b). Another way to increase the total surface area is by using a meandering structure. Several types of heaters with a meandering structures are implemented in the design. The simplest being a meander of $250 \mu \mathrm{m}$ long and a total of $250 \mu \mathrm{m}$ wide(figure $3.2 \mathrm{c} \mathrm{\&} \mathrm{d}$ ). The spacing between the different branches is identical to the line width. These meanders are extremely long and therefore have a large resistance. Meandering structures with a lower resistance are made by using a smaller number of lines or making the meander less wide. Figure 3.3a shows a vertical meander of 5 lines with a line width of $5 \mu \mathrm{m}$. In a horizontal meander shorter lines are used to decrease the total width and thus reduce the resistance of the meander (figure 3.3b). The total surface area of these smaller meanders is much smaller. In order to increase the surface area a number of these smaller meanders are positioned in parallel (figure $3.3 \mathrm{~d} \& \mathrm{e}$ ). This decreases the total resistance with respect to the large meander even further. Several more exotic structures were also fabricated and tested. A line with small fins (figure 3.4a) might improve the cooling after the heating pulse. 


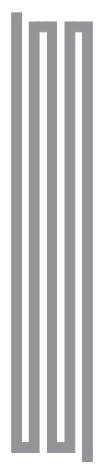

(a)

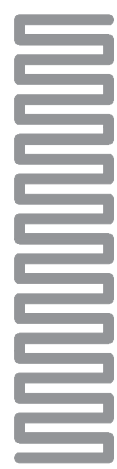

(b)

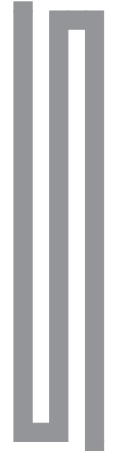

(c)

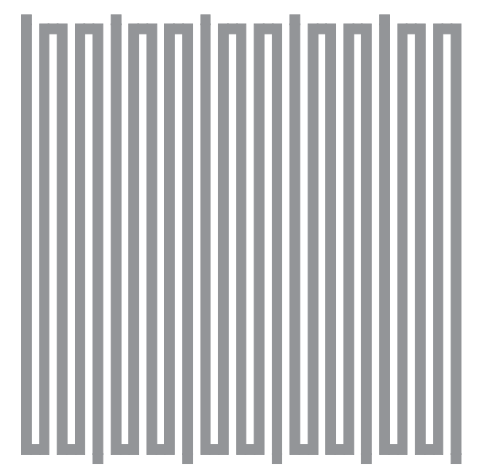

(d)

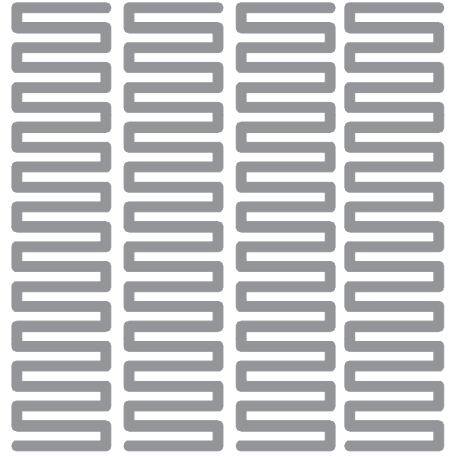

(e)

Figure 3.3: Different heater layouts; (a) a vertical meander with 5 lines of $5 \mu \mathrm{m}$, (b) a horizontal meander of $50 \mu$ wide and a line width of $5 \mu \mathrm{m}$, (c) a meander with 3 lines of $10 \mu \mathrm{m}$, (d) 5 vertical meanders in parallel, (e) 4 horizontal meanders in parallel.

A small square heater(figure 3.4b) has a relatively low resistance and large heating area. Instead of sharp corners sheets with rounded corners (figure 3.4c) might lead to a smoother transition from lead to actual heater. The heaters are not transparent and, especially for the sheets, bubbles formed on the heaters are hard to see due to a limited amount of light. Heaters with large holes (figure 3.4d) could solve this problem. Bubble nucleation at a pre-determined place can be achieved if almost al the energy is dissipated in on spot. Extremely non-uniform heater can be used in this case. Figure $3.4 \mathrm{e}$ shows a $50 \mu \mathrm{m}$ sheet with a narrow part of $5 \mu \mathrm{m}$ wide and $20 \mu \mathrm{m}$ long in the centre.

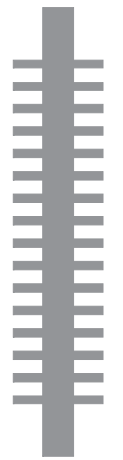

(a)

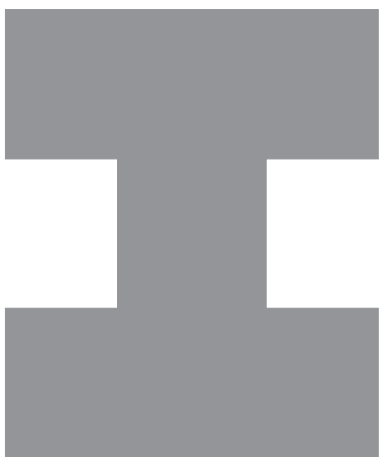

(b)

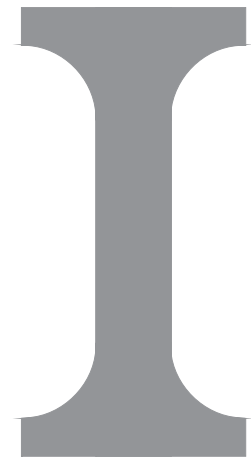

(c)

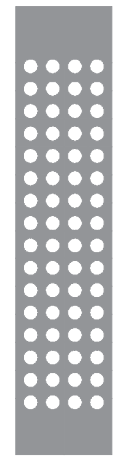

(d)

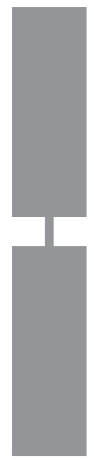

(e)

Figure 3.4: Different heater layouts; (a) a $20 \mu \mathrm{m}$ line with small cooling fins, (b) a square of $100 \mu \mathrm{m} \times 100 \mu \mathrm{m}$, (c) a $25 \mu \mathrm{m}$ sheet with rounded corners, (d)a $65 \mu \mathrm{m}$ sheet with $10 \mu \mathrm{m}$ diameter holes (e)a non-uniform heater with a small $20 \mu \mathrm{m} \times 5 \mu \mathrm{m}$ part.

The heaters are connected to four contact pads by four leads. Two wide leads are used to carry the current and two small leads to measure the potential difference across the heater. The current leads are made as wide as possible to reduce the resistance. A lower resistance will lead to less dissipation of energy in the leads and lower potential difference has to be applied across the contact pads to get the necessary potential difference across the heater. The voltage leads are used to measure the potential difference and should 
therefore have a high impedance. The voltage leads are therefore dimensioned as small as possible. Two designs of the leads were used and both are shown in figure 3.5. The heaters are situated in the gap between the top and bottom part. The changes to design 3.5a were made, because the position of the current lead was found to be important. In design $3.5 \mathrm{~b}$ the current leads are perpendicular to the heater and should lead to a more uniform current density.

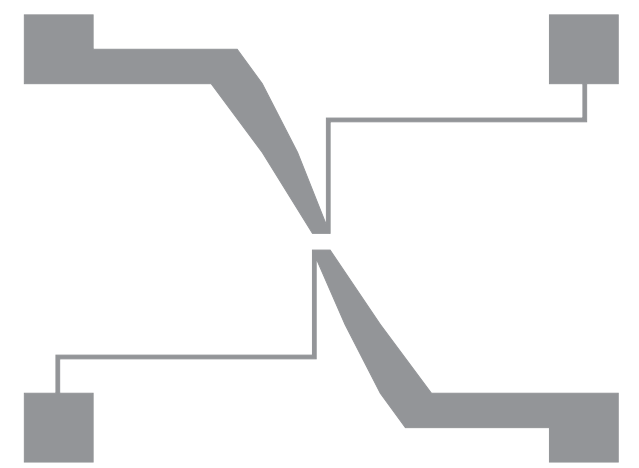

(a)

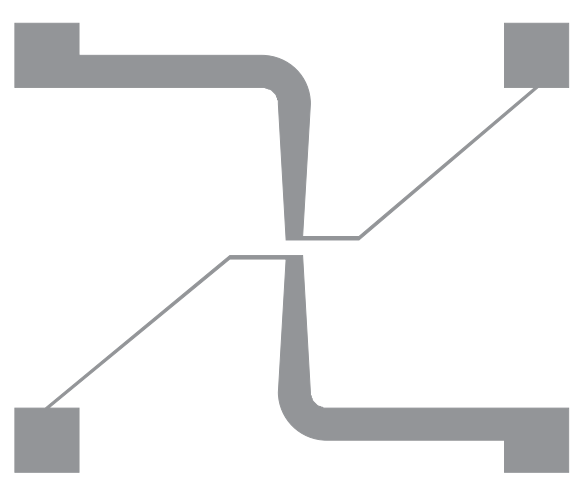

(b)

Figure 3.5: Two different design for the leads to the heater and electrical contact pads.

\subsubsection{Fabrication}

The heaters are fabricated using thin film deposition and lift off. The fabrication process starts with a clean Pyrex wafer. A layer of resist is spun onto the wafer. Two types of resist were used. First Olin Ti35 image reversal resist was used. The image reversal resist has the advantage that it has a negatively tapered profile after development. Unfortunately for several steps in the lithography, baking and development process it proved to be difficult to find the right process parameters. The use of the more standard Olin 907/17 resist, although it results in positive profiles, gave a more reproducible result. After development a thin metal film was deposited. For the cases where the image reversal resist was used a $10 \mathrm{~nm}$ titanium and a layer between $100 \mathrm{~nm}$ and $250 \mathrm{~nm}$ of platinum were evaporated with the Balzers BAK 600. Because the standard resist results in a positively tapered profile there will be hardly any difference between evaporation and sputter deposition. The use of the home-build sputter machine (Sputterke) was preferred in this case, simply because it takes less time to deposit the metal layers. For this machine a tantulum target was available and thus was used as an adhesion layer. The adhesion layer was again $10 \mathrm{~nm}$ in thickness and the platinum layer was also between $100 \mathrm{~nm}$ and $250 \mathrm{~nm}$. After metal deposition the actual lift off is preformed by ultra-sonic cleaning in acetone. This removes the resist, together with the parts of the metal that were not patterned by lithography. The results after lift-off are shown in figure 3.6. 


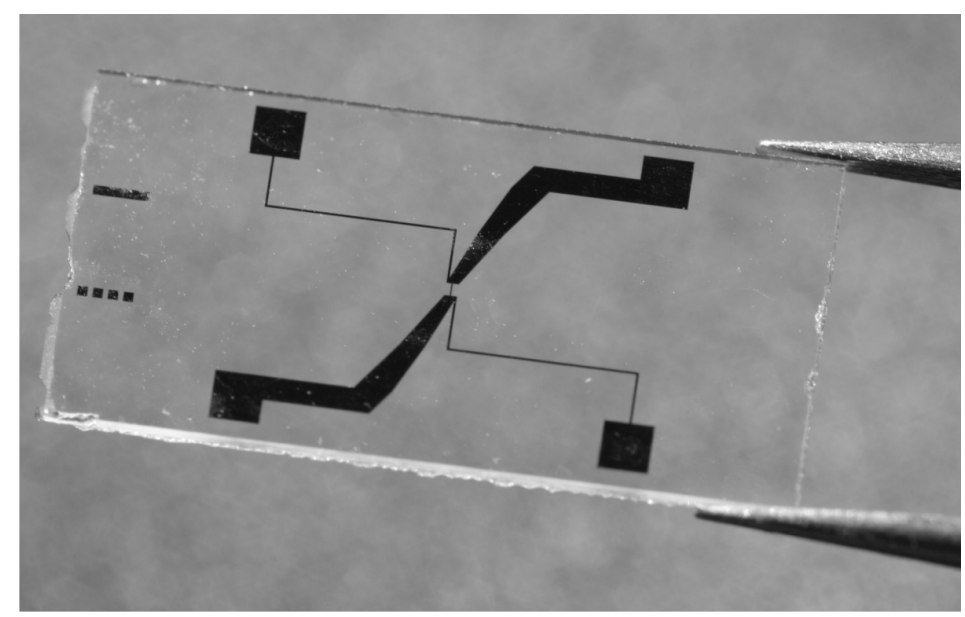

Figure 3.6: The heater and the leads on a pyrex substrate after lift-off.

\subsection{Fabrication by $\mathrm{KOH}$ etching}

The second part of the bubble actuator is made from a 4 inch (100)-oriented $525 \mu \mathrm{m}$ thick silicon wafer. After cleaning in fuming and boiling aqueous $\mathrm{HNO}_{3}$-solutions and an aqueous HF-solution the substrates are thermally oxidized (oxidation in $\mathrm{H}_{2} \mathrm{O}$ at $1150^{\circ} \mathrm{C}$ ) to achieve a $\mathrm{SiO}_{2}$-film thickness of $500 \mathrm{~nm}$. After this the wafers were coated with a Low Pressure Chemical Vapour Deposition (LPCVD) process using $\mathrm{SiH}_{2} \mathrm{Cl}_{2}$ and $\mathrm{NH}_{3}$ gas, resulting in low stress silicon-rich silicon nitride (SiRN) with a film thickness of $1 \mu \mathrm{m}$. For the explosive micro-bubble actuator SiRN was used as the material for the membrane. Silicon nitride was used, because the material has good elastic properties and is relatively strong. The second reason is that it can be used as a mask for $\mathrm{KOH}$ etching.

Both sides were coated in Olin 907/17 resist. On one side the resist will only serve as a protection layer during further processing. The other side is exposed to Ultra Violet light in a EVG 620 mask aligner. Further details on the mask design and details about the design of the channels, cavities and holes for electrical connection are discussed later. After baking and development exposed parts of the silicon-nitride and silicon-oxide are removed by reactive ion etching (RIE) with plasma containing $\mathrm{C}_{4} \mathrm{~F}_{8}$ and $\mathrm{CH}_{4}$ in a Adixen DE plasma-etcher. In the next step the wafer is cleaned and the native oxide layer is removed in a $1 \% \mathrm{HF}$ solution before it is submerged in a $25 \mathrm{wt} \% \mathrm{KOH}$ solution at $75^{0} \mathrm{C}$. After several hours of anisotropic etching of silicon the channel structure, the cavities and some acces holes are formed in the silicon. At the sites of the cavity and acces holes the $\mathrm{KOH}$ etches completely though the silicon substrate stopping at the silicon-oxide. After $\mathrm{KOH}$ etching the wafers have to be cleaned in a RCA-2 solution, which is a solution containing $\mathrm{HCL}: \mathrm{H}_{2} \mathrm{O}_{2}: \mathrm{H}_{2} \mathrm{O}$ in the volume proportion (1:1:5), to prevent contamination by the potassium ions in further process steps. After this step the silicon-oxide at the bottom is removed by wet-etching in a buffered HF solution. The top part of the microbubble actuator with the membranes, cavities and channels is now finished. 


\subsubsection{Mask design}

The fabrication of the top part of the bubble actuator by the use of $\mathrm{KOH}$ etching requires only one mask for patterning the channels and the cavities. Although the depth of the channels (between $20 \mu \mathrm{m}$ and $50 \mu \mathrm{m})$ and the cavity $(525 \mu \mathrm{m})$ are completely different both can be formed in the same process step by using the anisotropic etch characteristics of silicon in a $\mathrm{KOH}$ solution. The etch speed of crystalline silicon in a $\mathrm{KOH}$ solutions is different for different crystal orientations. The exact etch speed in the different directions depends on the temperature and composition of the $\mathrm{KOH}$ solution, but in general the $<100>$ and $<110>$ planes etch much faster than the $<111>$ plane. Because of the large difference the etching virtually stops at the $<111>$ planes. For a (100) oriented wafer this results in etch profiles such as shown in figure 3.7. In figure 3.7a the etch profile

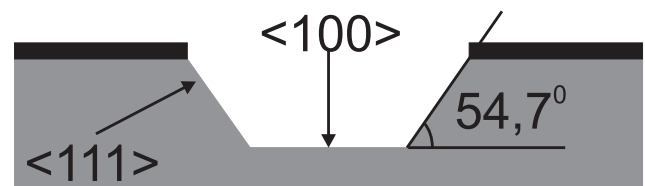

(a) Large pattern

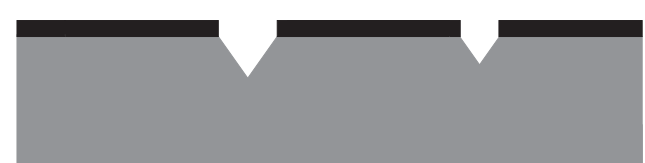

(b) Small patterns

Figure 3.7: Anisotropic etching of silicon in a $\mathrm{KOH}$ solution

for a relatively large pattern is shown. Still a large area with a $<100>$ plane is exposed and the edge depth is determined by the etch rate of the $<100>$ plane and the total etch time. Figure 3.7b shows the etch profile for a relatively small pattern. There is no $<100>$ plane exposed any more and the depth of the profile is mainly determined by the the profile width. Because the angle between the $<111>$ and the $<100>$ plane is always the same at 54,7 degrees one can calculate the pattern width needed to reach a certain depth or make a hole through a whole wafer with a certain width.

Etching a silicon wafer with a mask in $\mathrm{KOH}$ will always result in a undercut. The undercut is a result of etching in the direction parallel to the mask for this reason the final pattern in the silicon will always be larger than the original pattern in the masking material. The amount of undercut is highly dependent on the the mask pattern and its orientation with respect to the crystal planes of the wafer. For a (100) oriented wafer the edges parallel and perpendicular to the flat of the wafer(the [100] directions) show the smallest undercut. The speed is determined by the etch speed of the $<111>$ plane. Figure 3.8 shows the result after etching in $\mathrm{KOH}$ solution for a square (a) and a small rectangular (b) pattern. The gray part is the pattern of the mask and the dark part is under etched. If the mask is slightly rotated $(3.8 \mathrm{c} \& \mathrm{~d})$ due to a misalignment or a design with lines not perpendicular or parallel to the $<110>$ direction the under etch at certain places is much faster. The difference in etch speed of the undercut is maintained until the etch speed is limited by the $<111>$ plane in all directions. If one etches long enough, the pattern in silicon will thus always be made up out of the $<111>$ planes.

The corners in a mask design need special attention. Figure $3.9 \mathrm{~b}$ shows the undercut for concave and convex corners. The concave corners show a relatively small undercut, convex corners however etch much faster and the undercut is much larger. If convex corners are really necessary in a design one can use corner compensation $[98,99,100]$ to 


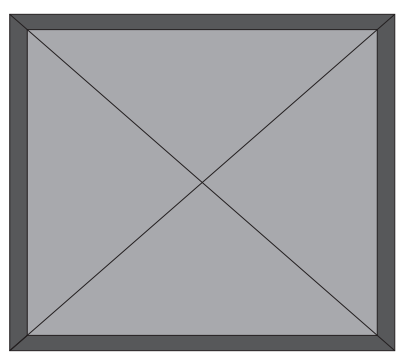

(a)

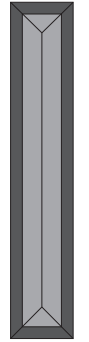

(b)

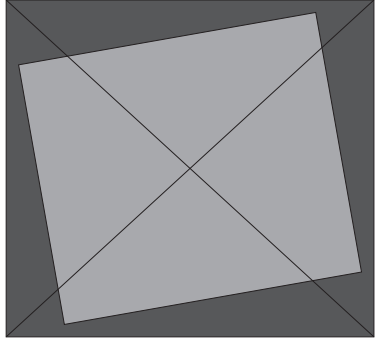

(c)

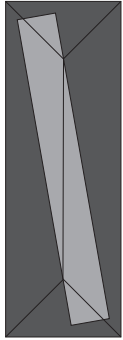

(d)

Figure 3.8: Undercut for a silicon wafer etched in $\mathrm{KOH}$ solution.

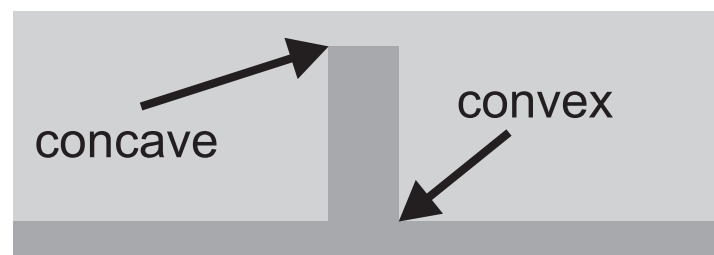

(a) Before etching

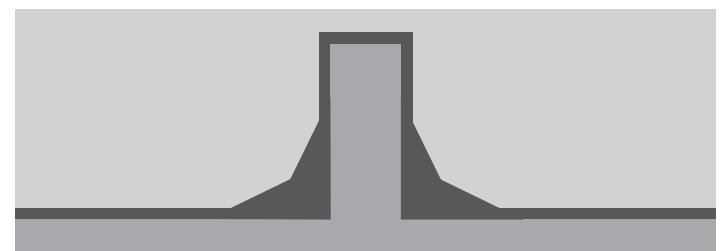

(b) After etching

Figure 3.9: The etching of convex and concave corners

create convex corners.

The micro-bubble actuator requires a membrane, a cavity, channels and holes for electrical connections. The use of $\mathrm{KOH}$ etching of silicon limits the geometry to square and rectangular membranes structures. The layout of a mask for single micro-bubble actuator is shown in figure 3.10 and contains two slits, which will form the channels, a square in the middle, which will form the cavity with a membrane, and 4 square at the sides , which will form the holes for electrical connections, in grey. The black line shows the size of a single sample. The layout of the actual mask contains 48 samples with different membrane and channel sizes.

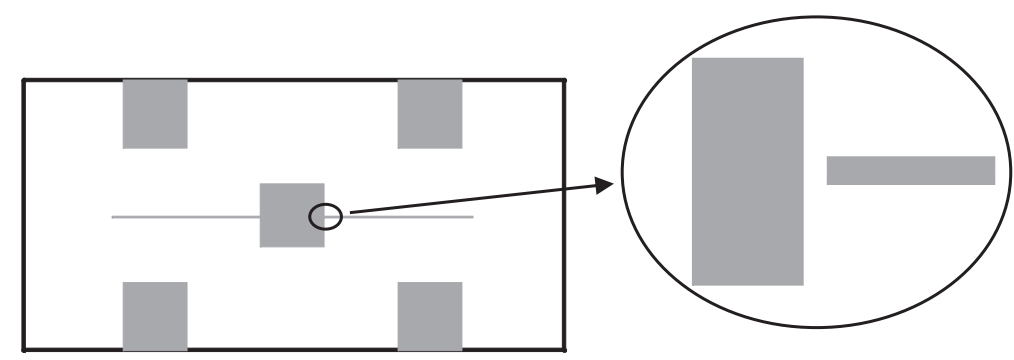

Figure 3.10: Mask design for $\mathrm{KOH}$ etching of a micro-bubble actuator and a zoom-in on the connection between the slits and the square. This connection will be become the connection between the channel and the cavity.

The size of the membranes as well as the size and shape of the cavities underneath the membrane are determined by the opening in the mask at the backside of the wafer and the wafer thickness. The opening in the mask needed to create a square membrane 
with a certain size $\left(a_{\text {membrane }}\right)$ can be calculated using:

$$
a_{\text {opening }}=a_{\text {membrane }}+2 h \tan \left(35.3^{0}\right)
$$

where $a_{\text {opening }}$ is the size of the sides of the opening in the mask and $h$ is the wafer thickness. The actual membrane will be slightly bigger due to the undercut. The size of the undercut depends on the total etch time. The etch speed in the [100] direction is about $1 \mu \mathrm{m}$ per minute and for a (100) oriented $525 \mu \mathrm{m}$ thick wafer it thus takes about 9 hours to etch through a wafer. Measurements on wafers etched for 9 hours show a undercut of about $10 \mu \mathrm{m}$.

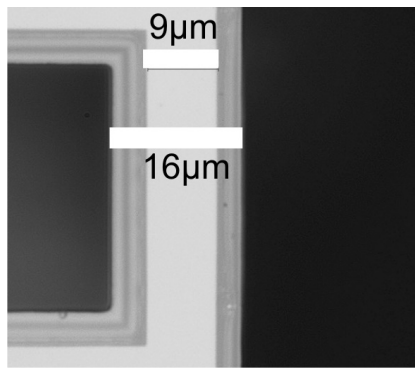

(a) Undercut after 3.5 hours

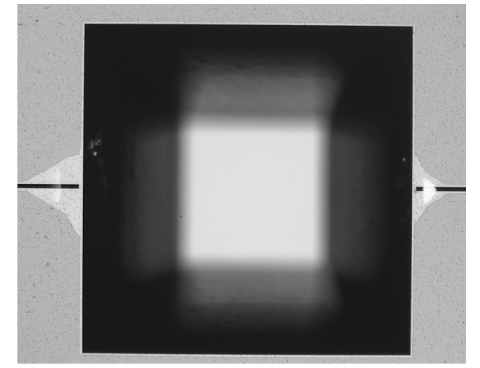

(b) Cavity after etching

Figure 3.11: Results of etching in a $\mathrm{KOH}$ solution. In (a) the under-cut is the dark gray part is equal to $3.5 \mu \mathrm{m}$ on both sides. In (b) the undercut is the light gray line surrounding the square and the two triangular shaped parts at the points were the channels(slits) and cavity (square) meet.

The hydraulic resistance of the fluidic channels is mainly determined by the length and area of its cross-section. A high hydraulic resistance limits the flow through the channels induced by the generation of bubbles. The channels should thus be relatively long and have a small cross-section in order to create a high hydraulic resistance. The cross-section of the channels has a triangular shape and the height is determined by the width of the slits in the mask and the total undercut. Because the width of the channels is relatively small the contribution of the undercut becomes more significant as the total etch times increase. Slits between $5 \mu \mathrm{m}$ and $40 \mu \mathrm{m}$ in width should after 9 hours of etching in $\mathrm{KOH}$ solution result in channels with a width of $25 \mu \mathrm{m}$ to $60 \mu \mathrm{m}$ and heights between $18 \mu \mathrm{m}$ and $42 \mu \mathrm{m}$. Connecting the channels to the cavity makes the use of convex corners inevitable. Creating a good compensation structure however is difficult and requires accurate timing of the etch process. In this case the exact geometry of the corners is not of high importance and a certain degree of undercut is still acceptable. The slow, but constant etch rate of the $<111>$ plane, can be used as a time-delay. The zoom-in on the mask design in figure 3.10 shows that the slits were not connected to the square openings in the mask, but a small distance of several microns was kept between the two. The distance was chosen such that just before the end of the etching process the undercut of the channel reaches the undercut of the cavity. At the moment the undercuts meet a convex corner is formed. This corner will etch quickly opening the connection between the channel and the cavity. Figure 3.11a shows the the undercut after 3.5 hours. The distance between the slit and 
the square opening in the mask is $16 \mu \mathrm{m}$ and the distance between the channel and the cavity still is $9 \mu \mathrm{m}$. The undercut is thus $3.5 \mu \mathrm{m}$ at both sides. After 9hours of etching a connection between the channel and the cavity is formed(figure 3.11b). The relatively large undercut in the convex corners clearly shows. Figure 3.11c shows a zoom of the area around the connection. The end of the channel has a conical shape and a rather rough surface.

\subsection{Fabrication with Reactive ion etching}

The use of a $\mathrm{KOH}$ solution to etch though a silicon wafer limits the design to square or rectangular channels, membranes and cavities. The same type of structures can also be formed by removing the silicon with deep reactive ion etching. In this case the silicon is bombarded with high energy ions created in a plasma. Under the right conditions physical and chemical processes result in the selective and highly directional removal of silicon. The use of a DRIE process to remove the silicon has a large impact on the fabrication process and the mask design. Like KOH etching the DRIE process can also be anisotropic, but there is almost no dependence on crystal orientation. The profile of the walls can be tuned from negatively to positively tapered by changing parameters such as the composition of the reactive gasses, temperature pressure and power. This makes it possible to create almost every imaginable membrane and channel geometry. Here it will be used to create circular membranes. The consequence of using DRIE process however is that a separate masks are needed to create the shallow channels and the deep cavities. Using two masks also implies extra lithography, baking and cleaning steps. Here the most important steps will be discussed. The first steps in the fabrication are identical to the ones described in previous section (3.3). A 500nm layer of thermal silicon-oxide and $1 \mu \mathrm{m}$ of LPCVD silicon-nitride are grown on a $525 \mu \mathrm{m}$ thick (100) oriented silicon wafer.

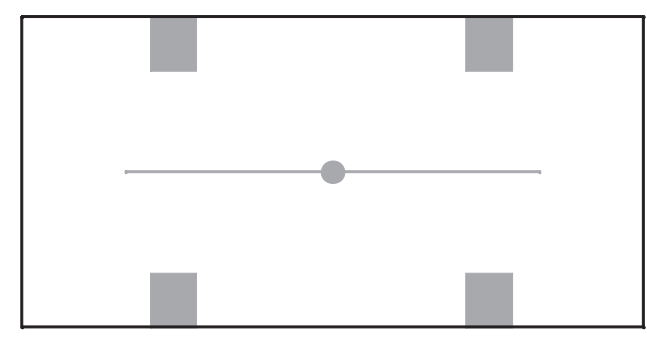

(a) First mask

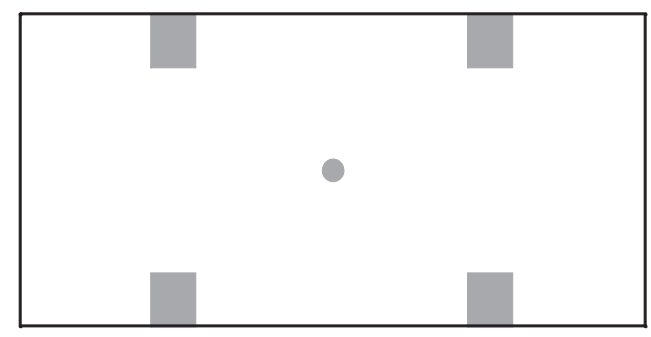

(b) Second mask

Figure 3.12: Masks used in the fabrication of the micro-bubble actuator using DRIE

A $3.5 \mu \mathrm{m}$ layer of Olin908/35 is coated on both sides and the back side is exposed using the mask shown in figure 3.12a. Besides the pattern for the channels the mask also contains the patterns for the cavity and electrical access holes. The silicon-oxide and silicon-nitride are locally removed using the Adixen DE plasma-etcher. In the next step the channel and the first part of the cavity and holes are formed by DRIE with a Adixen SE plasma-etcher. The Bosch-process, which is a process where an alternating process of deposition of a fluor-carbon protection layer and etching of silicon with $\mathrm{SF}_{6}$ results in 
deep structures with nearly vertical side walls, was used to a depth of about $50 \mu \mathrm{m}$ in the silicon. The channels are now finished and have to be protected in the next steps. This can be achieved by coating the the wafer in a $40 \mu \mathrm{m}$ thick layer of SU-8 photo-resist. The thickness of the layer needs to be thick enough to completely cover the channel, any exposure of the side-walls or edges will cause problems in further steps. The SU-8 can be used as normal negative photo-resist and a second mask shown in figure $3.12 \mathrm{~b}$ is used to pattern the cavity and the access holes again. A Adixen SE running a Bosch process again was used, but now etching was continued until the silicon-oxide layer at the other side of the wafer was reached. The $\mathrm{SU}-8$ is removed afterwards by pyranha $\left(\mathrm{H}_{2} \mathrm{SO}_{4}: \mathrm{H}_{2} \mathrm{O}_{2}(3: 1)\right)$ cleaning. The wafer with round membranes and $50 \mu \mathrm{m}$ deep channels with a rectangular cross-sections is now completed.

\subsection{The completed device}

The fabrication of micro-bubble actuator is completed by combining the silicon based top part and the pyrex based bottom part. Before the two parts can be merged a few fabrication steps on the separate parts still have to be preformed. A connection between the channels and the outside world is established by drilling two holes in the pyrex part. The holes are drilled by powder blasting and situated at the area were the channels will end. The pyrex part is now finished and an image of the part is shown in figure 3.1a.

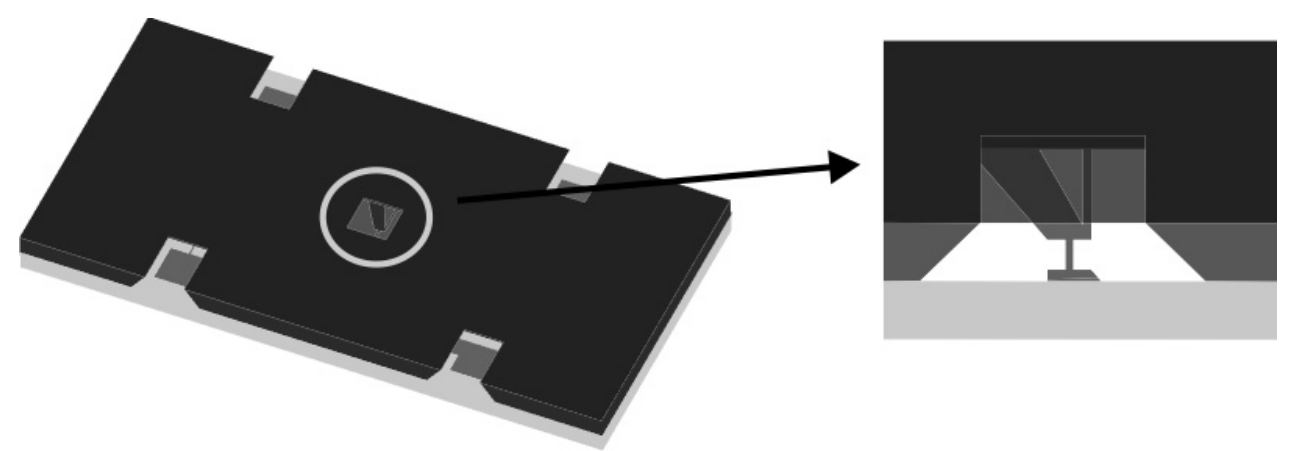

Figure 3.13: The completed micro bubble actuator and a zoom-in under an angle of a cross-section through the cavity

The silicon part (figure 3.1b) has a silicon-nitride top layer on both sides. In order to merge the silicon part and the pyrex part by complete wafer bonding the composition of the silicon-nitride layer first has to be changed. The silicon-nitride deposited by LPCVD deposition does not bond to pyrex. However, if the silicon-nitride is thermally oxidized (oxidation in $\mathrm{H}_{2} \mathrm{O}$ at $1150^{\circ} \mathrm{C}$ ), a layer of silicon-oxynitride is formed. This layer can form a strong bond with pyrex. The silicon part and pyrex part are anodically bonded at $400^{\circ} \mathrm{C}$ and $1000 \mathrm{~V}$. After bonding the wafer is diced into $15 \times 7.5 \mathrm{~mm}^{2}$ samples. Dicing the wafer in smaller samples also opens the four cavities located at the edges of single sample. This makes it possible to make an electrical connection between the contact pads and the measurement equipment. The completed micro-bubble actuator and a zoom on the 
cavity are shown in figure 3.13. Figure 3.14 shows the fabricated devices from the front and backside.

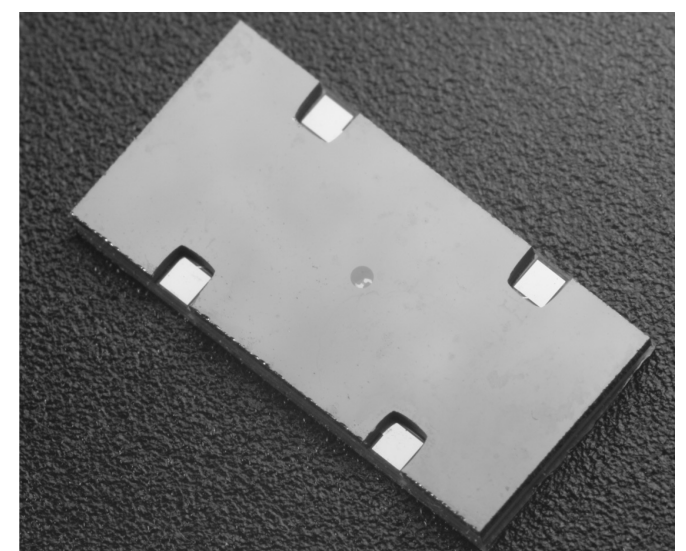

(a) Front

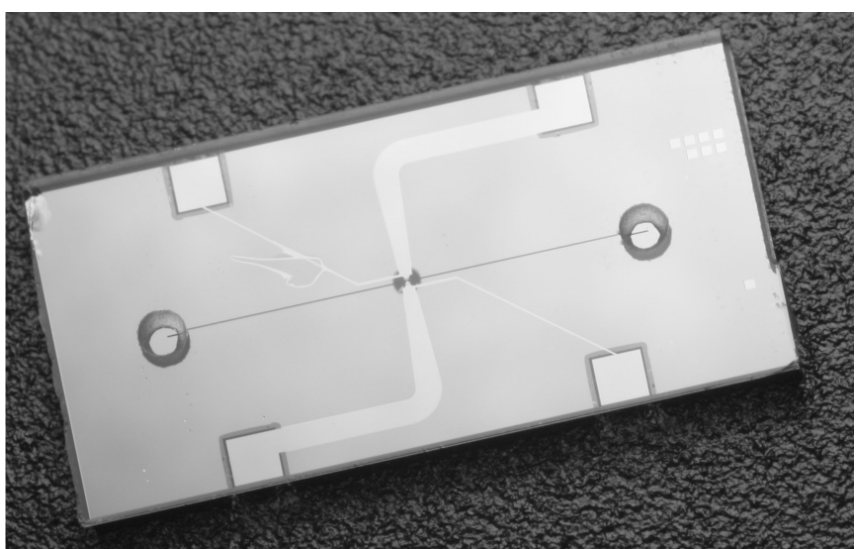

(b) Back

Figure 3.14: Photos of the front and back side of the device after fabrication.

\subsection{Experimental setup}

The filling holes of the actuator were connected to two tubes via a perspex sample holder and some rubber o-rings. The inlet-tube was connected to a pressure sensor and to a syringe-pump and the outlet was connected to the outside world through a valve. A syringe filled with degassed ethanol was loaded in to the syringe pump and the cavity was flushed for several minutes to remove air-bubbles trapped in the cavity. After this the syringe pump is stopped and the outlet valve is closed, sealing in the the liquid in the cavity.

The four electrical contact pads are connected to the electronics. The heater is heated due to joule heating in microsecond pulses. The pulses are generated by a home-made pulse generator. During the heating pulse the current and potential difference across the heater are monitored with an oscilloscope. The current through the heater is determined by measuring the potential difference across a fixed resistance in series with the heater. The applied power and average heater resistance were calculated from the potential difference and current. In order to determine the average heater resistance during the off-state of the heating pulse, a small probe current was applied. This current was large enough to measure the average resistance, but did not result in a serious heat dissipation in the heater.

Two different types of measurements were conducted. The bubble nucleation growth and collapse were imaged by stroboscopic imaging. A microscope was equipped with a CCD-camera and three high-intensity LED were placed in the eye-pieces and underneath the sample. The three LEDs enabled us to illuminate the sample from several angles and made sure that a sufficient intensity of light was generated to produce a clear image from a single exposure. This was an important requirement, because the phenomena were 
not expected to be completely reproducible. The home-made pulse generator was used to provide the heating pulse and trigger the light pulse. An image of the setup and a zoom-in on the sample holder with the heater is shown in figure 3.15.

The dynamic membrane deflection was measured with a MSA 400 laservibrometer from Polytec. The sample with the perpex sample holder were placed underneath the laservibrometer. The homemade pulse generator provides the heating pulse and the trigger pulse. During the vibrometer measurements the heater temperature was also monitored. The Polytec also has a white light interferrometer to measure the static deflection of the membrane.

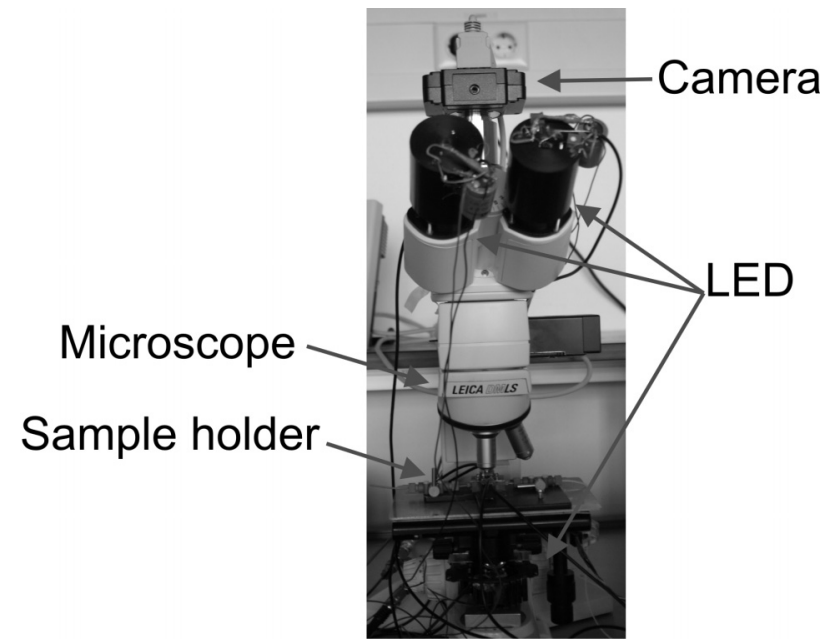

(a)

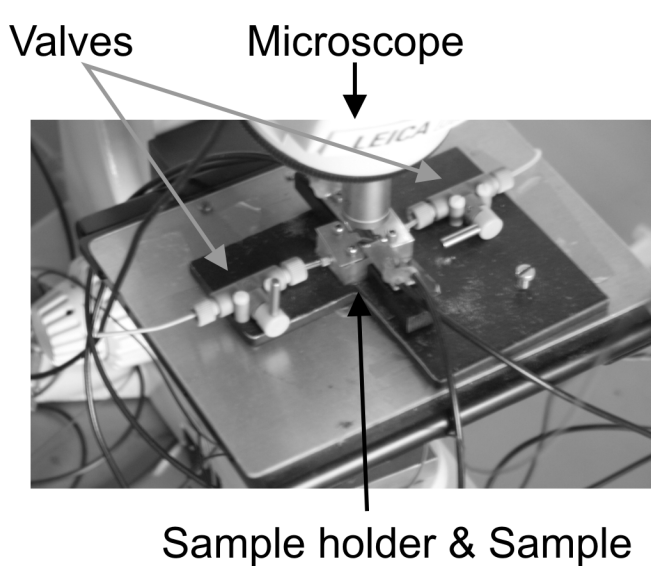

(b)

Figure 3.15: Image of the experimental setup (a) and a zoom-in of the sample-holder with the valves and a sample (b). 


\section{Chapter 4}

\section{Bubble dynamics}

The bubble generation and the bubble dynamics were studied by resistance thermometry and stroboscopic imaging. These processes are complex and influenced by a large number of parameters. The effects of parameters, such as the cavity pressure, heating power, heater size and repetition frequency on the nucleation, growth and collapse of the bubbles are investigated. 


\subsection{Introduction}

The actuator uses explosive evaporation to generate a bubble. The dynamics of this process are very complex and still a topic of research. The process can be divided in five different stages; heating of the liquid, bubble nucleation, bubble growth, bubble collapse and liquid cooling.

In order to get insight in these stages several measurement methods can be used. One important method is visual observation of the bubbles. The substrate as well as the membrane material are transparent and this enables us to image the bubbles. Because the events take place in microseconds either high-speed cameras or stroboscopic imaging techniques are needed to visualize them. The second method to study the processes is by monitoring the heater temperature with resistance thermometry. With this method the temperature of the liquid close to the heater and the substrate temperature can be estimated. This information can give more insight in the thermodynamics of the different steps in the process.

The bubble nucleation will be influenced by the heating power, length of the heating pulse and heater geometry. These parameters will also have a large effect on the further development of the bubble. The pressure inside the cavity will have a considerable influence on the bubble growth and collapse. The bubble actuator is should be able to function at repetition frequencies of several $\mathrm{kHz}$. Therefore influence of repetition frequency on the bubble nucleation, growth and collapse was investigated.

\subsection{Stroboscopic imaging}

The process of explosive evaporation occurs in microseconds on a micrometer sized heater. To visualize these events a microscope and special imaging techniques are required. Highspeed cameras are capable to record up to 25 million frames per second, which means an image every 40ns. These cameras however are expensive and a much cheaper way to image microsecond events is by stroboscopic imaging. Stroboscopic imaging uses short light pulses to illuminate the sample and a normal CCD-camera to record an image. The sample is only exposed during the light pulse and kept in the dark for the remaining time. The image taken by the CCD-camera around the same time as the light pulse will only show the situation during this light pulse.

One of the problems of high speed imaging is that short light pulses and/or shutter times are used and images are often under-exposed. To increase the exposure either a more intensive light source must be used or one can combine several images taken at the same moment in time. The last solution only works for reproducible phenomena, because every image has to be identical.

In this research stroboscopic imaging was used to study the explosive evaporation. A complete sequence of bubble nucleation, growth and collapse was recorded by increasing the time difference between the start of the heating pulse and light pulse for every image. The intensity of a single light pulse was sufficient to get a good image from a single exposure. Each image shown in this chapter therefore corresponds to single event and therefore to a different bubble. 


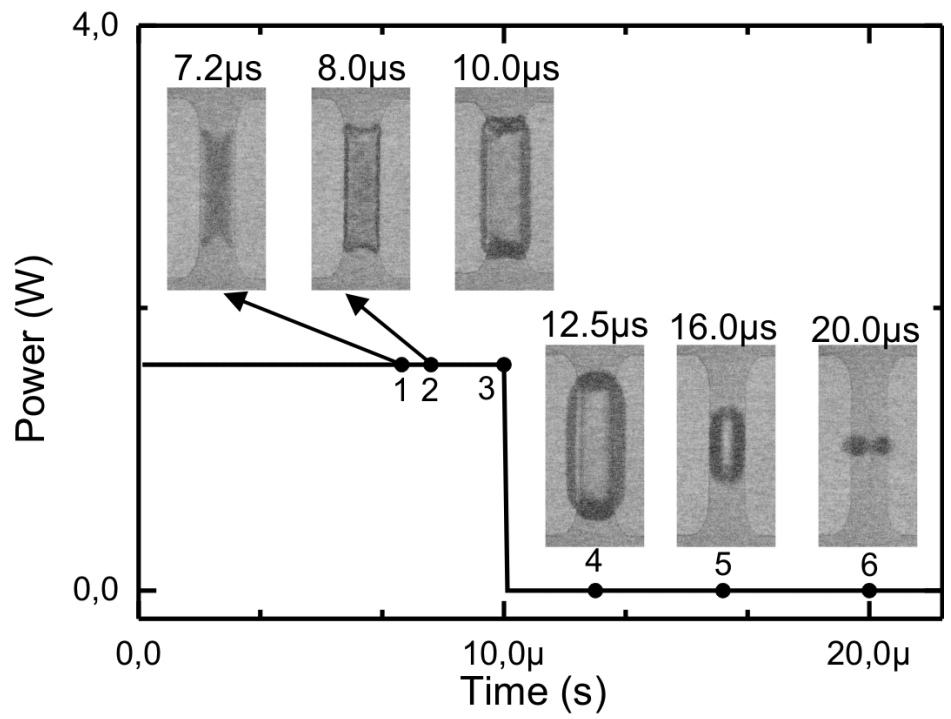

Figure 4.1: Imaging of bubble nucleation, growth and collapse with stroboscopic imaging. The applied heating power is shown as a function of time.

Figure 4.1 shows such a sequence for $10 \mu$ s heating pulse at an applied power of $1.6 \mathrm{~W}$. The length of the light pulses was set at 200ns. The bubbles nucleate around $7.2 \mu \mathrm{s}$ and at $8.0 \mu \mathrm{s}$ a vapour sheet covers a large part of the heater. At $10 \mu \mathrm{s}$ the heating is stopped, but the bubble still grows. The shape of the bubble becomes more rounded and at $12.5 \mu \mathrm{s}$ it reaches a maximum. From this point on the bubble shrinks and finally a collapse takes place around $20 \mu \mathrm{s}$. After this the bubble quickly disappears and the actuator is ready for a new pulse. The whole sequence at this heating power and pulse length thus takes less than $25 \mu$ s.

\subsection{Resistance Thermometry}

Monitoring the temperature during the explosive boiling can give a lot of information on the dynamics of the process. Ideally one wants to measure the temperature at several locations in the liquid, in the substrate and on the heater. Due to the small size of the heater and the small penetration depth of the heat this is nearly impossible. We however were able to monitor the heater temperature by resistance thermometry. In resistance thermometry the linear dependence of the resistance of certain metals on the temperature is used to determine the temperature [95]. Equation 4.1 can be used to determine the temperature $(T)$ from the resistance $(R)$.

$$
\frac{R}{R_{0}}=1+\alpha\left(T-T_{0}\right)
$$

The temperature coefficient $(\alpha)$ and the resistance $\left(R_{0}\right)$ at a known temperature $\left(\mathrm{T}_{0}\right)$ can be determined by calibration measurements. The temperature coefficient only depends on the material properties, but the exact value can change with process parameters during deposition. The measured temperature will always be the average heater temperature. 
Local variations in temperature across the heater will result in local resistance changes. With the four probe measurement, however, only the average resistance of the heater can be determined. It is therefore possible that some points of the heater have a higher temperature, while other places have a lower temperature.

\subsubsection{Calibration}

In order to determine $R_{0}$ and $\alpha$, a sample was put into a metal container together with a PT-100 thermocouple. The container was slowly heated from room temperature to $120^{\circ} \mathrm{C}$ and the resistance of the heater and thermocouple were measured using an Agilent 34970 data acquisition switch unit in four wire sensing mode. In figure 4.2 the resistance for

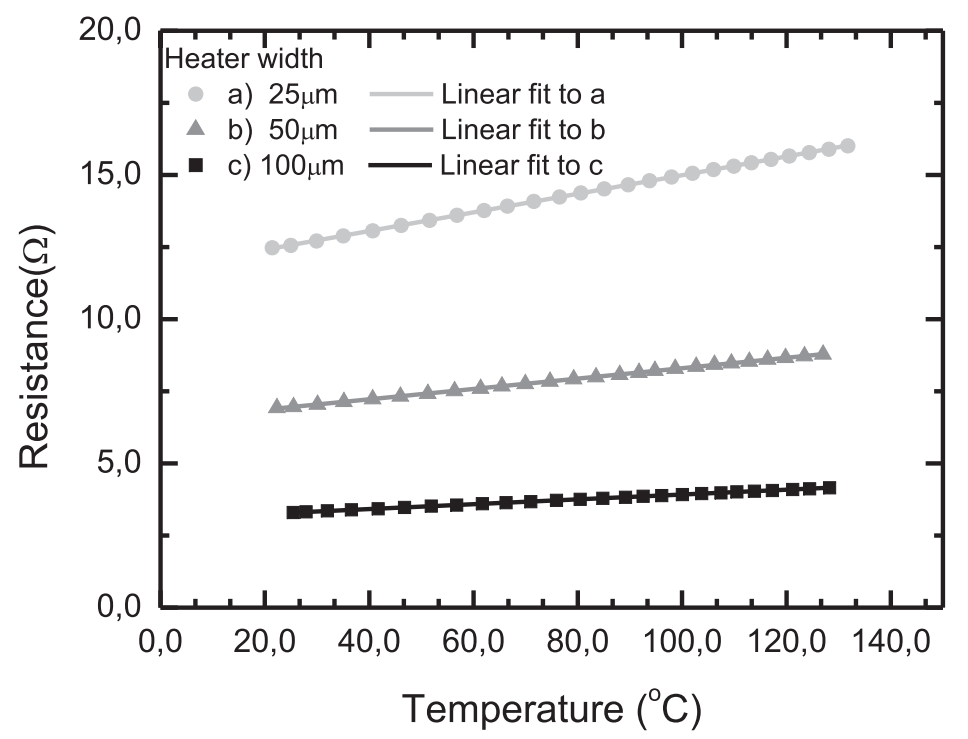

Figure 4.2: Resistance of the heater as a function of temperature for heaters with a width of 25,50 and $100 \mu \mathrm{m}$.

three different heaters is plotted as a function of temperature measured with the PT-100 thermocouple. All the heaters have an identical geometry(figure 3.4c), but the widths are $25 \mu \mathrm{m}, 50 \mu \mathrm{m}$ and $100 \mu \mathrm{m}$ respectively. The temperature coefficient $(\alpha)$ is given by the slope. The initial resistance $\left(R_{0}\right)$ is equal to the resistance at $T=0^{\circ} \mathrm{C}$.

While the range is smaller than the temperatures reached in the experiment, it is known that the resistivity of platinum is very close to linear over a wide range [95] and therefore it is likely that the extrapolation of equation 4.1 is sufficiently accurate.

\begin{tabular}{|r|c|c|c|}
\hline heater & $25 \mu \mathrm{m}$ & $50 \mu \mathrm{m}$ & $100 \mu \mathrm{m}$ \\
\hline$\alpha(1 / \mathrm{K})$ & 0.00273 & 0.00272 & 0.00270 \\
\hline$R_{0}(\Omega)$ & 11.8 & 6.50 & 3.08 \\
\hline
\end{tabular}

Table 4.1: Temperature coefficient and initial resistance for three different heaters

The calculated values for the temperature coefficient and the initial resistance are given in table 4.1. As expected the temperature coefficient is almost the same for all the 
heaters, because this value is determined by the heater material. The initial resistance scales with the width of the heater, because for these heaters the thickness and length are the same for the different heaters.

\subsection{Bubble nucleation}

One of the most important phases during explosive boiling is the bubble nucleation. In section 2.3 the theory on this phase was discussed. Here the results of the measurements on the bubble nucleation are shown. Stroboscopic imaging and resistance thermometry were done simultaneously. Every image corresponds to a single event and for every event the current and voltage difference were monitored. In order to get a more accurate temperature measurement the average over a large number of events was taken.

\subsubsection{Imaging}

Figure 4.3 shows a series of images for an applied power of $0.49 \mathrm{~W}$ and a pulse duration of $200 \mu \mathrm{s}$. In this case a light-pulse of $1 \mu \mathrm{s}$ and a $0.1 \mathrm{~Hz}$ repetition frequency were used. The first image is taken $143 \mu \mathrm{s}$ after the start of the heating pulse and there is a difference of $2 \mu \mathrm{s}$ between each image. In the third image a spherical vapour bubble can be seen. The nucleation thus takes place around $147 \mu \mathrm{s}$. For the last two images we see that the bubble is situated at the same place for separate measurements. The nucleation thus took place at the same site for separate measurements. This suggest either growth of a preexisting vapour bubble or heterogeneous nucleation from a defect or cavity.
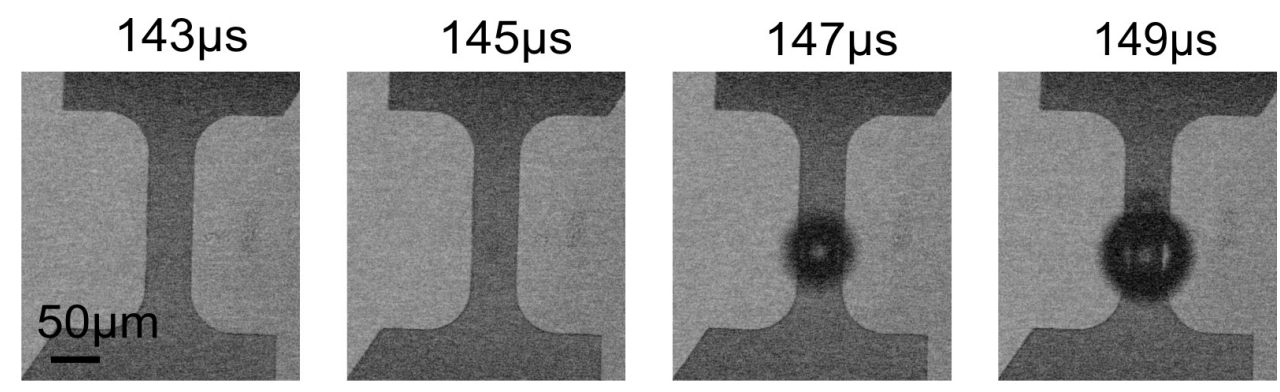

Figure 4.3: Images of bubble nucleation in ethanol at a heating power of $0.49 \mathrm{~W}$

For an applied power of $3.7 \mathrm{~W}$ the images are shown in figure 4.4. The duration of the heating pulse was set to $10 \mu$ s to prevent damage of the heater. The first image was now taken $1 \mu \mathrm{s}$ after the start of the heating pulse and the difference between each image was set to $1 \mu \mathrm{s}$. The third image shows a vapour sheet covering the narrow part of the heater. The bubble nucleation thus occurred around $3 \mu$ s after the start and the vapour sheet suggest spontaneous nucleation.

\subsubsection{Average heater temperature}

Figure 4.5 shows the average heater temperature measured during a $20 \mu$ s heating pulse of $1.2 \mathrm{~W}$ applied power. In this case we averaged over 32 measurements to increase the 

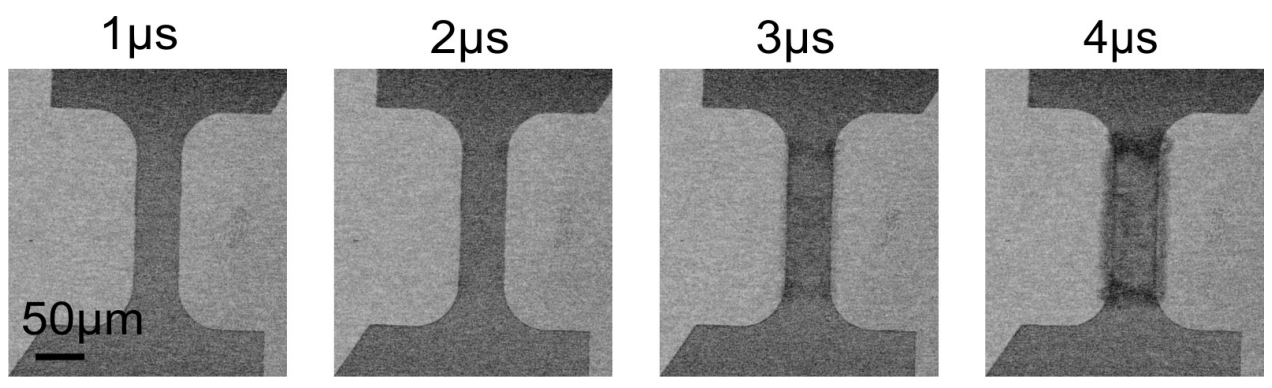

Figure 4.4: Images of spontaneous nucleation at 3.7W heating power

accuracy and the repetition frequency was $1 \mathrm{~Hz}$. The time between each pulse is thus $1 \mathrm{~s}$, which should be enough to ensure that all bubbles had dissolved again and the liquid was again at ambient temperature. At $13 \mu \mathrm{s}$ a change in slope can be observed. This change corresponds to the time of nucleation. This was verified by the images made with stroboscopic imaging with light pulses of $1 \mu \mathrm{s}$. Here a vapour film appeared at $13 \mu \mathrm{s}$ after the start of the heating pulse. The insets in figure show the heater before nucleation and at $13 \mu \mathrm{s}$. The average heater temperature at the moment of nucleation is about $180^{\circ} \mathrm{C}$.

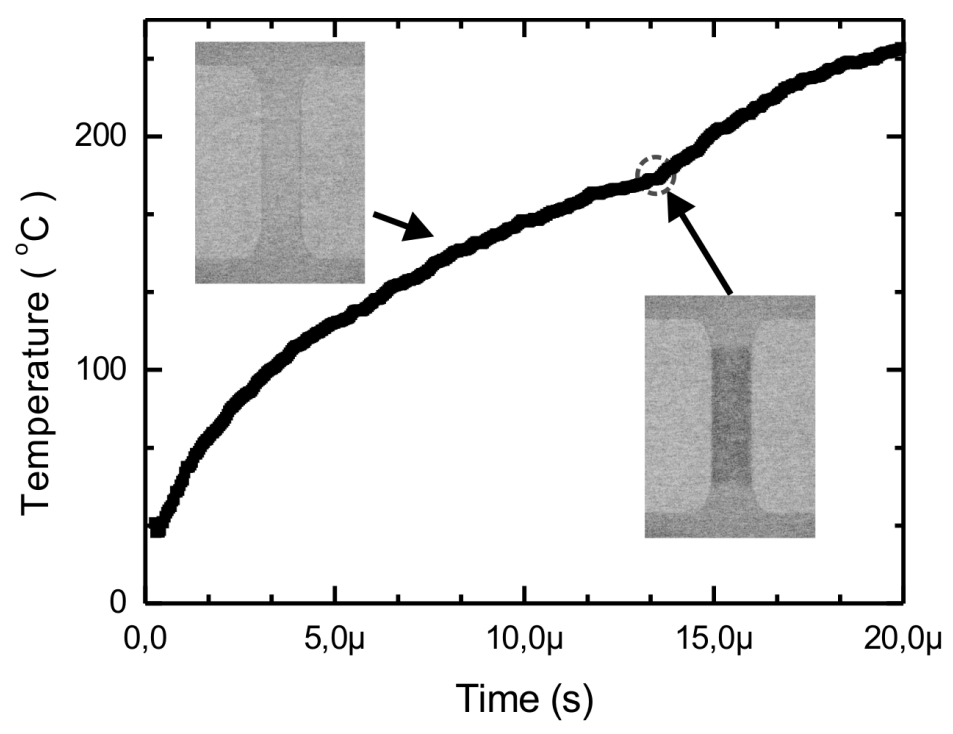

Figure 4.5: Heater temperature during the heating pulse for an applied power of $1.2 \mathrm{~W}$. The insets show images taken before and after nucleation.

\subsubsection{Influence of applied power on the bubble nucleation}

Figure 4.6 shows the instant of nucleation for different applied powers. In this case the stroboscopic imaging was used to determine the moment of nucleation. Between $0.4 \mathrm{~W}$ and $4 \mathrm{~W}$ the time, before bubble nucleation can be seen, decreases from $180 \mu \mathrm{s}$ to $3 \mu \mathrm{s}$ after the start of the heating pulse. In section 4.4.1 two different regimes were distinguished. For a heating power below $0.8 \mathrm{~W}$ we see that a single spherical bubble is formed on the central part of the heater. At heating powers above $1.3 \mathrm{~W}$ spontaneous nucleation occurs; 


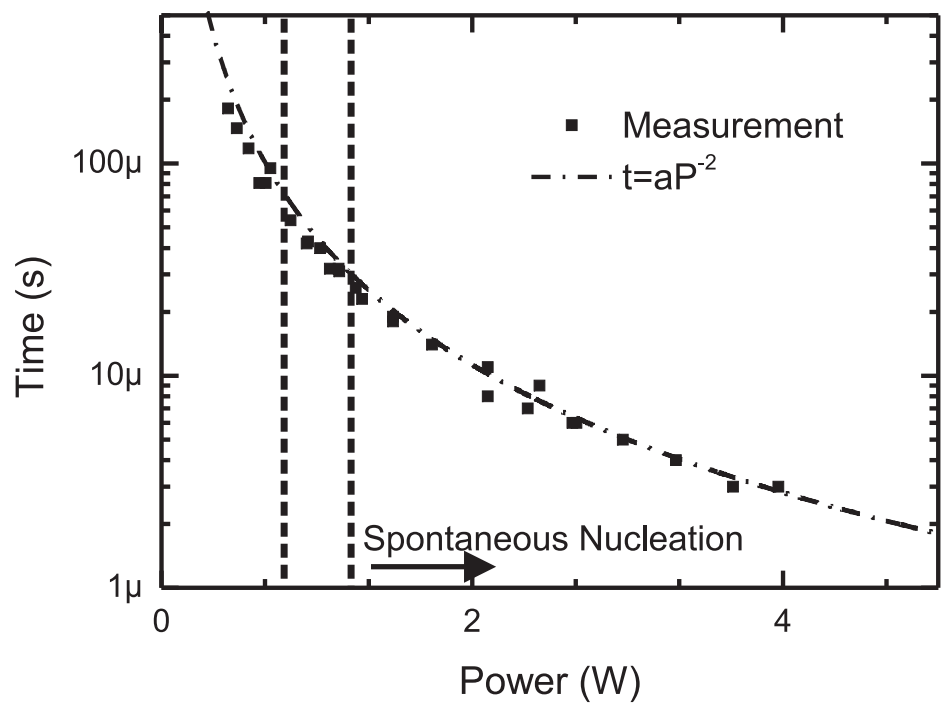

Figure 4.6: Measured bubble nucleation time for different applied powers.

a large number of bubbles nucleate at the same time across the heater. These bubbles almost instantaneously coalesce into a thin vapour film covering the heater. Between $0.8 \mathrm{~W}$ and $1.3 \mathrm{~W}$ spherical bubbles grow from multiple nucleation sites indicating a transition between the two regimes.

Figure 4.6 also shows the result for a fit to the following equation:

$$
t=\frac{a}{P^{2}}
$$

This equation is nearly identical to the equation 2.24. Except in this equation the heatflux $(q)$ is replaced with the heating power $(P)$ and the constant $C$ is replaced by $a$. The model is in good correspondence with the measured nucleation times. A value of $4.5 \times 10^{-5} \mathrm{~W}^{2} \mathrm{~s}$ for the constant $a$ was determined from the measured data points. This constant is mainly determined by the material properties of the liquid and the area of the heaters.

A difference between the two nucleation regimes can also be witnessed in the time traces of the average temperature. Figure 4.7 shows the average heater temperature for a relatively low heating power of $0.23 \mathrm{~W}$ and a relatively high heating power of $2.7 \mathrm{~W}$. For the low heating power we see a sudden decrease in temperature at $370 \mu$ s at a heater temperature of $170^{\circ} \mathrm{C}$. An image taken at this moment shows a single bubble on the central part of the heater. The sudden decrease in temperature is the result of nucleation at relatively low heat-fluxes. In this case the heat, which is needed to evaporate the liquid and to form a bubble, is extracted from the heater. This will result in a temperature drop at the heater surface. For a heating power of $2.7 \mathrm{~W}$ we see a change in slope of the curve at $2.9 \mu \mathrm{s}$ at a heater temperature around $185^{\circ} \mathrm{C}$. The image taken at that time shows a vapour film covering the heater. Here the slope change in the temperature curve is the result of a change in heat-conduction at the heater surface. The thermal conductivity of the liquid is several orders higher than the thermal conductivity of the vapour. At the moment of nucleation, liquid on the heater evaporates and the heater will be covered by 


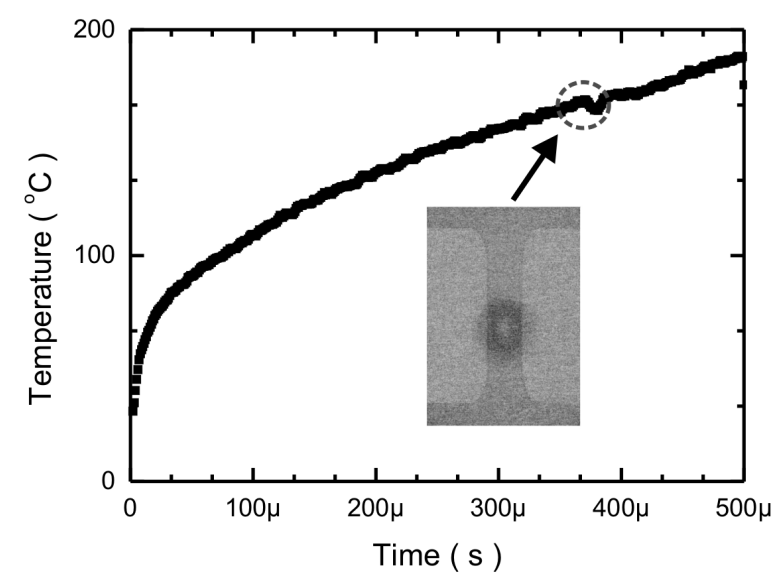

(a) $0.23 W$ applied power

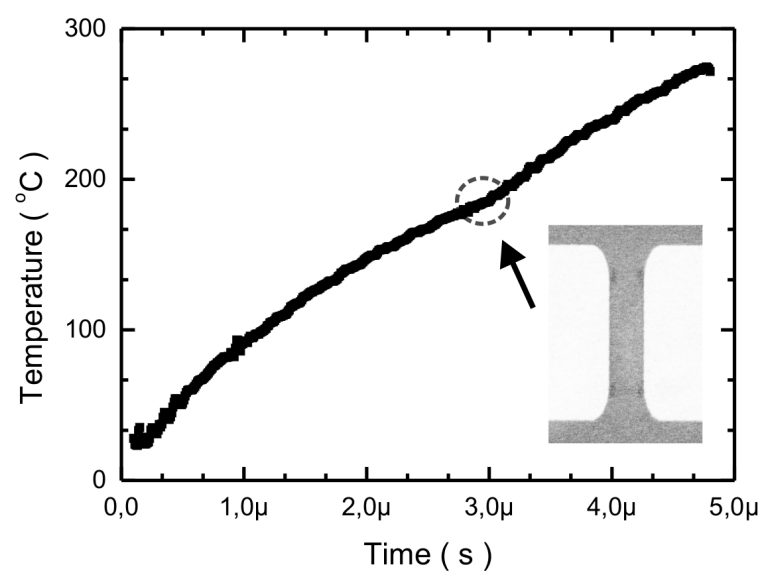

(b) $2.7 \mathrm{~W}$ applied power

Figure 4.7: Heater temperature during the heating pulse at two different applied powers. The insets are images taken at the time of nucleation.

a vapour layer. This change of thermal conductivity at the heater surface decreases the heat-transport from the heater and a faster rise of the heater temperature will be the result.

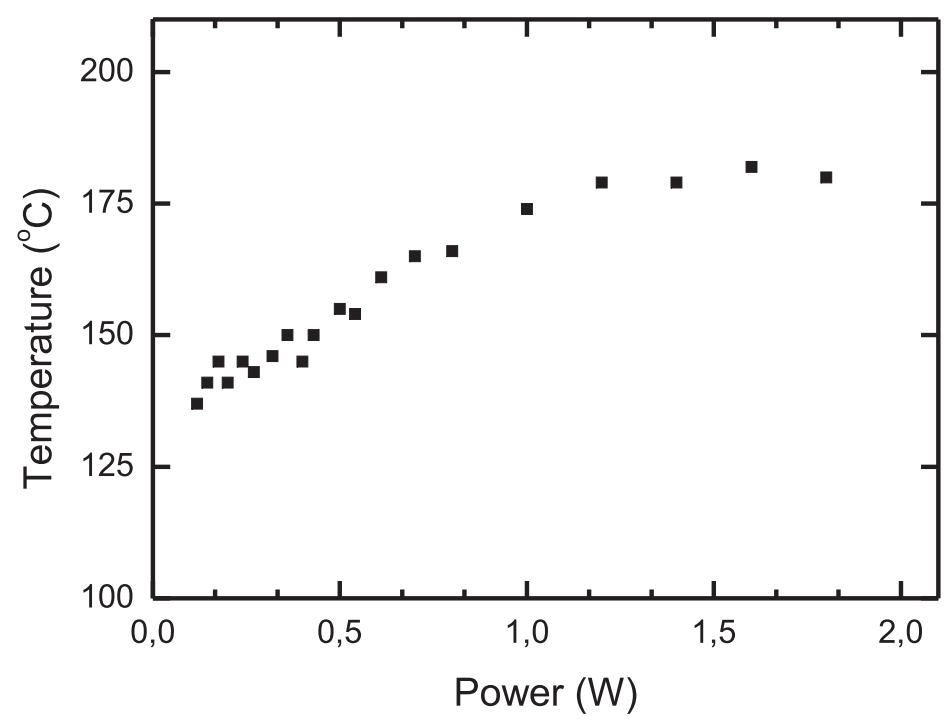

Figure 4.8: Nucleation temperature as a function of power for a $200 \mu \mathrm{mx} 25 \mu \mathrm{m}$ heater

The nucleation temperature changes with the applied power. In the single bubble regime, thus at relatively low heating powers, the nucleation temperature is expected to be lower than the spontaneous nucleation regime. Figure 4.8 shows the nucleation temperature against the applied power for a $200 \mu \mathrm{mx} 25 \mu \mathrm{m}$ heater. The nucleation temperature is determined from temperature-time curve. At low heating power the nucleation temperature is equal to the temperature just before the first temperature decrease. At intermediate heating power there is no decrease and the temperature at the slope change 
is used. For high heating powers the slope change becomes harder to determine. In those cases the time of nucleation was determined by stroboscopic imaging and the temperature corresponding to this time was determined from the temperature-time curve. As expected, the nucleation temperature increases with applied power. At low powers the nucleation temperature increases with power until a limit of $180^{\circ} \mathrm{C}$ is reached around $1.2 \mathrm{~W}$. A further increase in heating power does not lead to a significant increase in nucleation temperature. A similar behaviour was detected by Iida et al. [59], although the nucleation temperature measured here is lower than the value found by Iida. The limit in the temperature is the kinetic limit of superheat discussed in section 2.3.2. The uncertainty in the measured temperature for this measurement is about $20^{\circ} \mathrm{C}$, which is rather large. The origin of this uncertainty and the consequence for the temperature measurements are discussed in the next section.

The total energy $E$ needed to create a bubble is determined by the total input power $(P)$ and by the length of the heating pulse $\left(t_{\text {pulse }}\right)$ and is given by

$$
E=P t_{\text {pulse }}
$$

if this equation is combined with equation (4.2) this results in

$$
E=b \sqrt{t}
$$

The energy thus should increase with the square root of the nucleation time. This is

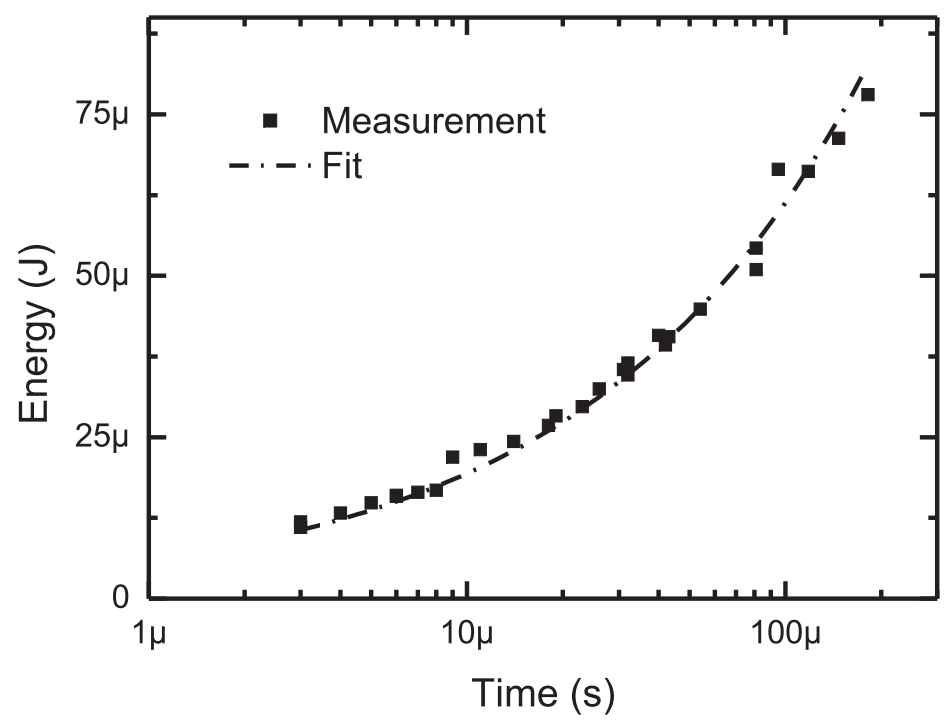

Figure 4.9: Energy input as a function of nucleation time. The measured data was fitted to equation (4.4)

also shown in figure 4.9, where the energy needed per pulse is shown as a function of the nucleation time. Equation 4.4 is used for fitting and a value of $6 \times 10^{-3}$ with an error of $7 \times 10^{-5}$ was found for $b$. Although the power to generate a bubble increases with a decrease in nucleation time, the total energy per pulse decreases. For bubble nucleation at $100 \mu \mathrm{s}$ a power of $0.65 \mathrm{~W}$ is needed, this corresponds with an energy of $65 \mu \mathrm{J}$ per pulse. 
In order to get nucleation at $3 \mu$ s only $12 \mu \mathrm{J}$ has to be supplied. The applied power however has to be $4 \mathrm{~W}$ during these $3 \mu \mathrm{s}$. At shorter pulse length less energy is needed to create a bubble at the end of the pulse. But then again shorter pulses will lead to smaller bubbles, because a smaller area is heated before bubble nucleation takes place.

\subsubsection{Reproducibility}

The goal of this project was to create a fast and powerful actuator. The previous section has shown that it can produce explosive bubbles within a few microseconds. But if one wants to use these bubbles as the driving force in an actuator, the generation has to be highly reproducible in time, size and place.
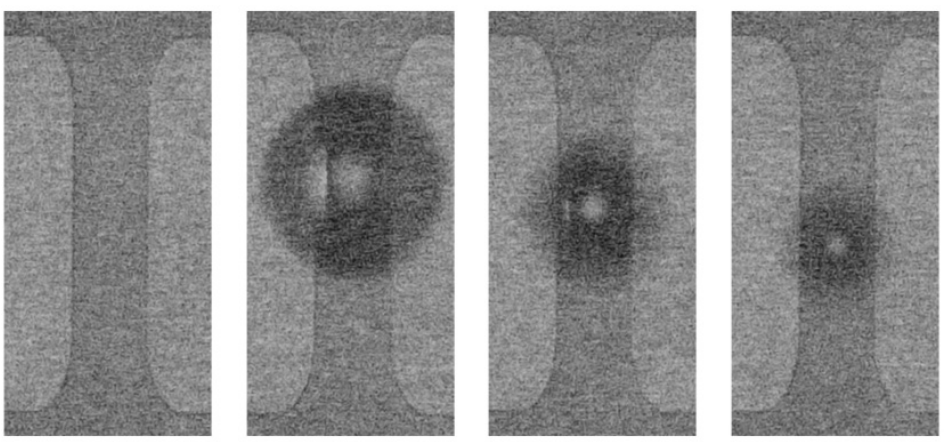

Figure 4.10: Images taken at the end of a $500 \mu$ s heating pulse of $0.2 \mathrm{~W}$.

Figure 4.10 shows 4 images taken at the end of a $500 \mu$ s heating pulse. The applied power was increased until nucleation takes place, in this case $0.2 \mathrm{~W}$. The pulse length of the light pulse was $2 \mu \mathrm{s}$. The first image shows no bubble. The other three show a bubble, but the bubbles are different in size and are located on a different part of the heater. It looks like the bubbles are in different stages of their development. A small difference in nucleation time explains this difference. At these relatively low heating powers the nucleation rate is low. Because the nucleation is a random process, small differences of less than a percent in nucleation time can result in a difference in nucleation time of more than $2 \mu$ s. This means a bubble appears in one image and does not appear in another one taken at the same time. The large difference in bubble size also demonstrates the large growth speed directly after nucleation. The radius of the largest bubble shown here is estimated at $25 \mu \mathrm{m}$. The time of growth cannot be determined with a high accuracy from these images, but is estimated at several microseconds. The growth speed will therefore be on the order of $10 \mathrm{~m} / \mathrm{s}$. Hong et al. [65] calculated the speed of the bubble interface in water heated under conditions almost identical to the ones in this research and found a value around $20 \mathrm{~m} / \mathrm{s}$ for the velocity in the z-direction during the growth. Zhao et al. [73] estimated the speed of growth of a bubble in water at $10 \mathrm{~m} / \mathrm{s}$ to $15 \mathrm{~m} / \mathrm{s}$ from measurements with an acoustic pressure transducer. Although water was used in both experiments the values are in the same range as the estimated growth speed in ethanol found from this research.

In figure 4.114 images taken at the end of a $3 \mu$ s heating pulse are shown. Again a $2 \mu \mathrm{s}$ light pulse was used and the power was increased until bubbles could be seen at the 

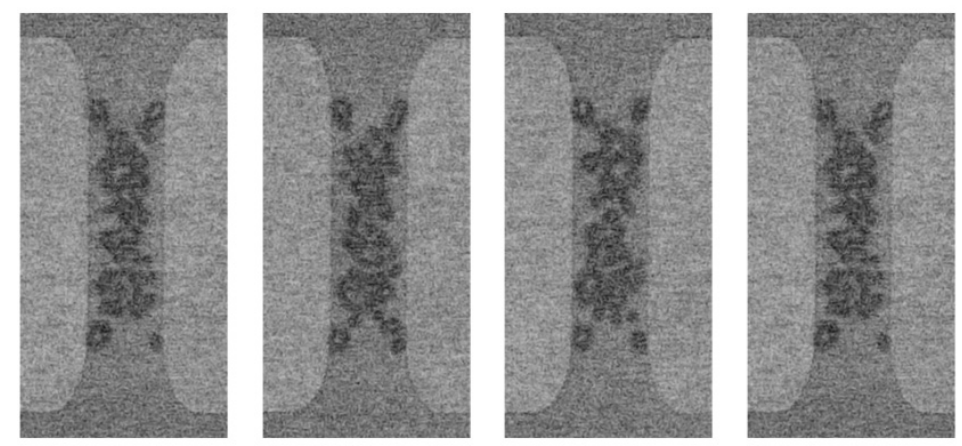

Figure 4.11: Images taken at the end of a $3 \mu \mathrm{s} 2.5 \mathrm{~W}$ heating pulse.

end of the heating pulse. In this case a power of $2.5 \mathrm{~W}$ was needed to get nucleation. All four images are almost identical. The bubbles all have about the same size and are spread over the same part of the heater. Some small differences in the exact spots of the bubbles however do appear. This clearly shows the high reproducibility of the spontaneous nucleation. Due to the high-heat fluxes a large number of bubbles appear almost at the same time and all the bubbles will thus have about the same size in all images. The small differences in the exact spots on the heater occur because of the randomness of the nucleation.

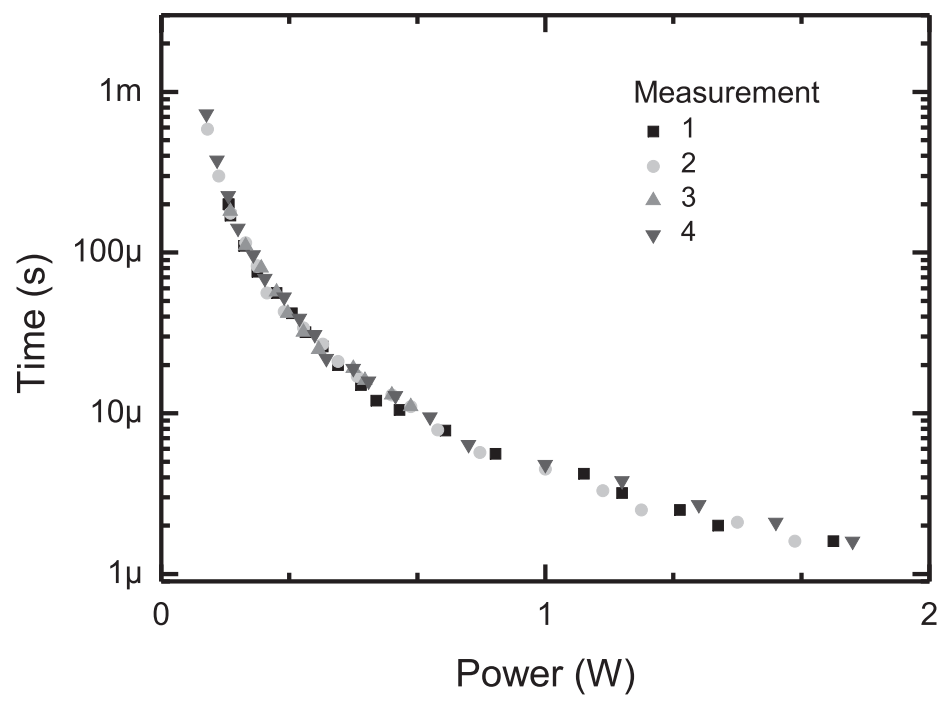

Figure 4.12: The measured nucleation time for four identical measurements.

The reproducibility in nucleation time can also be seen from figure 4.12. This figure shows the nucleation time as a function of applied power. The nucleation time was determined by stroboscopic imaging and the same measurement was repeated four times. For all the measurements the nucleation times are almost identical only small differences occur, but these are well within the error range of the time measurement.

During these measurements the average heater temperature was also monitored. Although the resistance change and thus the temperature change during a single pulse can 
be measured quite accurately, the uncertainty in the absolute temperature is rather large. This large uncertainty originates form the fact that the temperature is not measured directly, but is calculated from the potential difference across the heater and the potential difference across a fixed resistance in series with the heater. The combination of several small errors can lead to large changes in the calculated resistance and heater temperature. There is a large number of possible sources that can cause these errors. The main sources of errors in this case are:

- Drift in the off-set of the oscilloscope. Especially after a change in measurement range a rather large change in the off-set was found.

- Limited accuracy of the oscilloscope at high potential differences.

- Changes in the resistance of connections to the sample or connections between wires.

- Variation in the heater properties, such as the initial resistance or temperature coefficient

Furthermore the initial resistance and temperature coefficient of the heater are calculated from calibration measurements. These measurements have a much higher accuracy, but small errors in the initial resistance and temperature coefficient can lead to much larger errors in the calculated temperature, especially at high temperatures.

Several measures were taken to reduce the measurement uncertainty. The oscilloscope was instructed to preform a self-calibration on a regular basis and a change of range during a series of measurements was prevented as much as possible. The initial resistance of the heater and fixed resistance were measured before every measurement. These actions decrease the uncertainty in the absolute temperature slightly, but still the uncertainty will be around $10 \%$ of the calculated temperature. This is a rather high value, but recently published articles on comparable measurements by Romera-Guereca et al. [101] and $\mathrm{Xu}$ et al. [51] report an uncertainty of $6^{\circ} \mathrm{C}$ and $6 \%$ respectively. Some changes in the measurement setup and heater design should make it possible to reach a comparable accuracy. Moreover in most cases a highly accurate absolute temperature meaurement is not important. An accurate knowledge of the time that an event, such as bubble nucleation or collapse, takes place or the temperature chance during or between events can be sufficient and will provide enough information. The uncertainty in time is estimated at about $1 \%$ and the uncertainty in temperature change is about $5 \%$.

\subsection{Different heater geometries}

The influence of the applied power on the nucleation time was determined by stroboscopic imaging for several different types of heaters varying in size and geometry. Figure 4.13 shows the different heater layouts used in the measurements. The power as a function of nucleation time is shown in figure 4.14a. For all the heaters an increase in power results in a decrease in nucleation time. The meander (figure 4.13c) needs significantly more power to produce a bubble than for example a fin (figure 4.13b ). To determine whether this is 


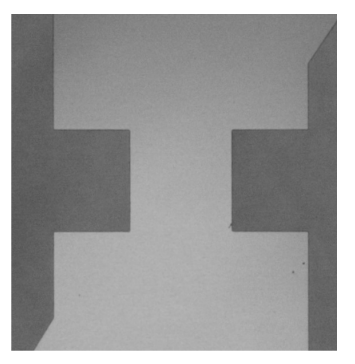

(a)

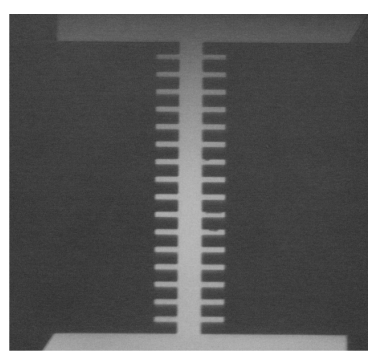

(b)

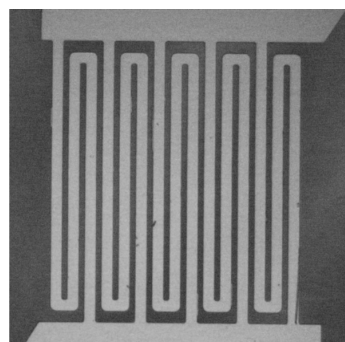

(c)

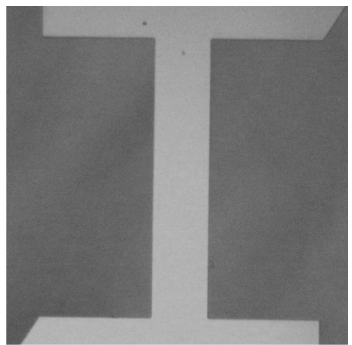

(d)

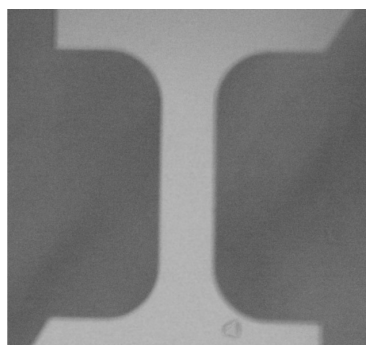

(e)

Figure 4.13: Different heater geometries: A square of $100 \times 100 \mu \mathrm{m}^{2}$ (a); A line of 20x250 $\mu \mathrm{m}^{2}$ with small cooling fins (b); 5 parallel $10 \mu \mathrm{m}^{2}$ wide meanders (c); A sheet of $50 \mathrm{x} 250$ $\mu \mathrm{m}^{2}$ (d) and a sheet of $50 \times 250 \mu \mathrm{m}^{2}$ with rounded corners(e).

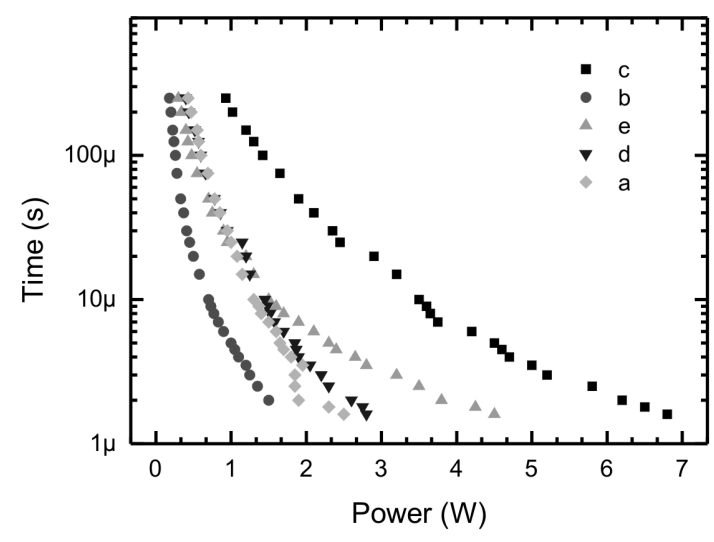

(a) Different applied powers

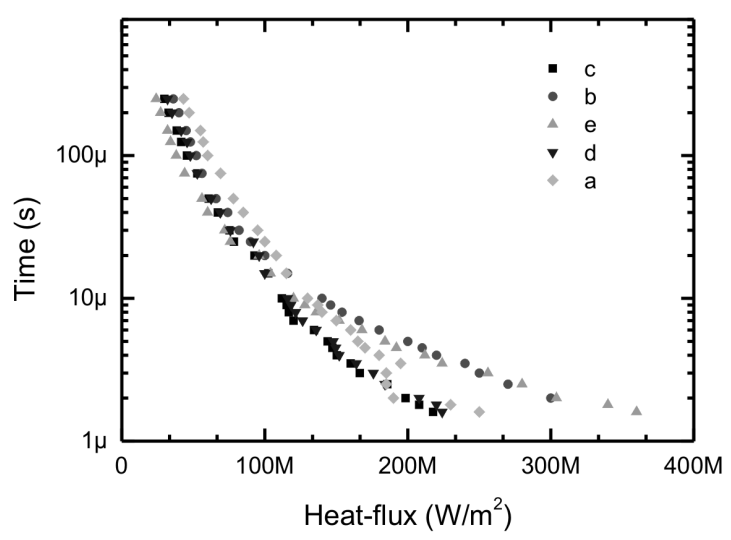

(b) Different heat-fluxes

Figure 4.14: The bubble nucleation time for different geometries determined by stroboscopic imaging. The letters correspond with the geometries shown in figure 4.13. 
only due to the difference in heater area or due to other parameters involved, it is better to look at the applied heat-flux (figure 4.14b).

At low heat-flux the nucleation time is almost identical for the different geometries, although small variations do exist. The differences are probably the result of uncertainties in the heater area and uncertainties in the calculated heating power. For low heatfluxes the geometry of the heater thus has no significant influence on the nucleation time. However, as the heat-flux increases, the first bubbles start to appear later for the fin
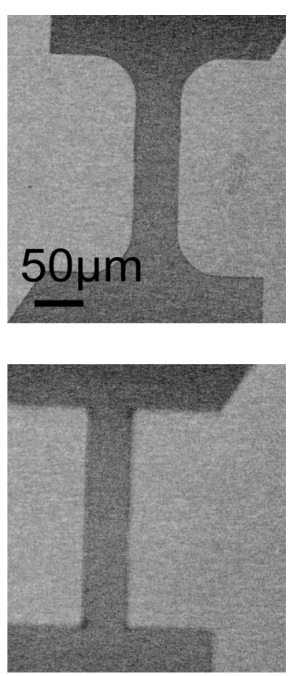

$1 \mu \mathrm{s}$
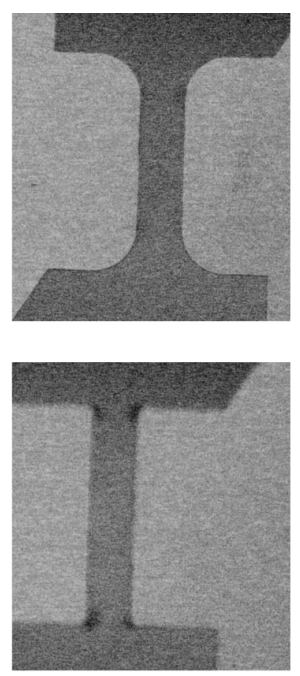

$2 \mu s$
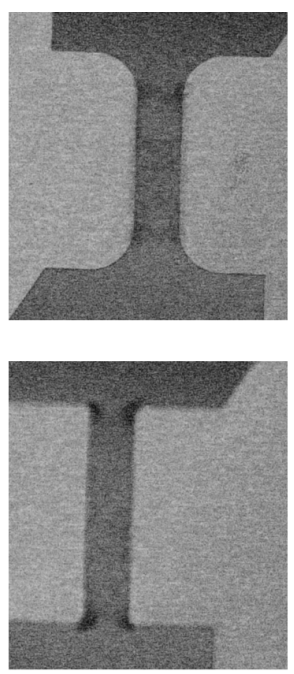

$3 \mu \mathrm{s}$
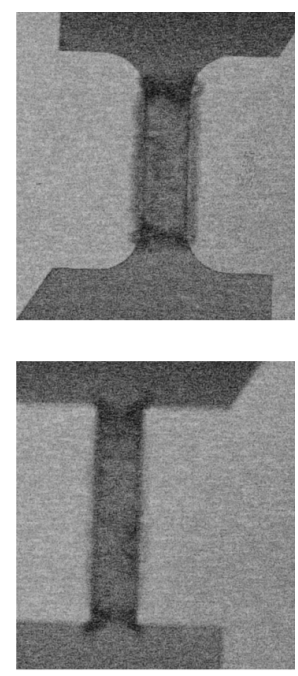

$4 \mu s$

Figure 4.15: Images of bubble nucleation at $3.7 \mathrm{~W}$ on a heater with rounded corners (top) and on a heater with sharp corner (bottom).

(figure4.13b) and the sheet with rounded corners (figure 4.13e). Especially the difference in nucleation time between the sheet with sharp corners (figure 4.13d) and the sheet with rounded corners is peculiar. The area of both heaters is nearly the same and there only is a slight difference in geometry. Still at high heat-fluxes the bubbles clearly nucleate earlier for the heater with the sharp corners.

\subsubsection{Early nucleation}

A closer look at the images (figure 4.15) taken around the time of nucleation for an applied power of $3.7 \mathrm{~W}$ shows that for the heater with the sharp corners small bubbles appear in the corners $1 \mu \mathrm{s}$ after the onset of the heating pulse. For the heater with the rounded corners a vapour sheet is formed immediately after nucleation at $3 \mu \mathrm{s}$ and no difference in nucleation time and time of vapour sheet formation can be detected. For the heater with the sharp corners nucleation takes place earlier and in the form of small bubbles at few distinct sites. However, if we continue the heating, a vapour sheet is again formed after $3 \mu \mathrm{s}$. This can also be concluded from figure 4.16, where we see that nucleation occurs earlier for heaters with sharp corners for heat-fluxes above $100 \mathrm{MW} / \mathrm{m}^{2}$. For both types of heaters the vapour sheets are formed around the same time after onset of heating. This early nucleation creates small bubbles, which will generate only a weak pressure pulse. Heating has to be continued to get a vapour sheet across the entire heater. 


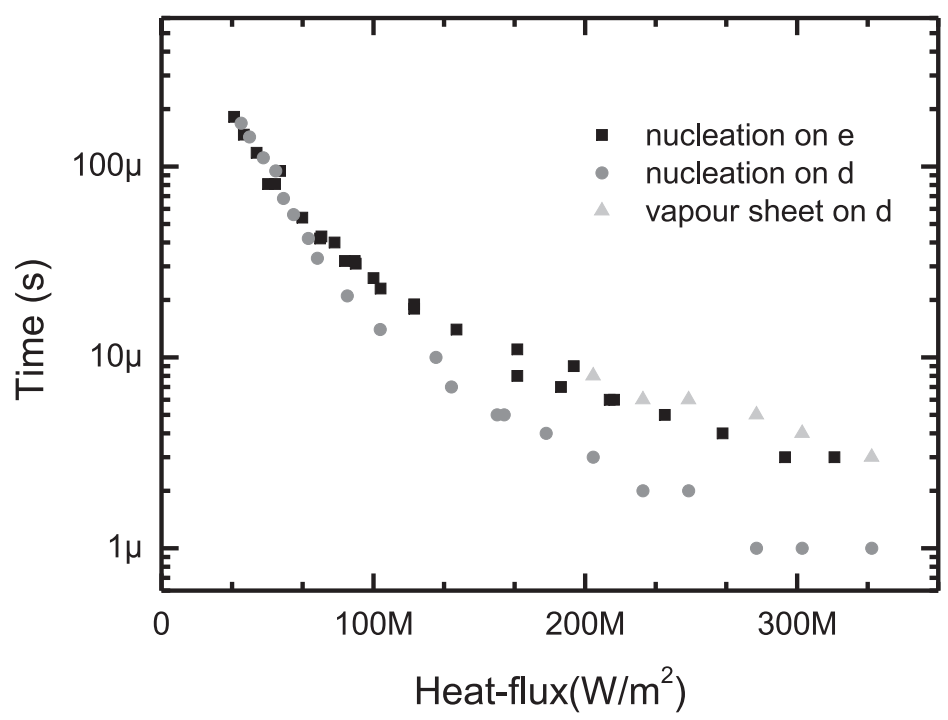

Figure 4.16: Time of bubble nucleation on heaters with rounded and sharp corners and the time of vapour sheet formation for the heater with sharp corners. For the heater with rounded corners a vapour sheet is formed immediately after nucleation and nucleation and vapour sheet formation time are identical.

\subsubsection{Current density simulation}

A reason for the nucleation in the corners of the heater is occurrence of a non-uniform current density across the heater during the heating pulse. Near the corners the current density is expected to be higher and thus the temperature at these sites will rise faster. The nucleation temperature will be reached earlier and the first bubbles will appear at the locations were the current density and thus the heat-flux is highest.

Figure 4.17 shows results of a simulation with Flex-PDE, which numerically calculated the current density profile of the heater, for a few of the geometries. The current density is clearly much higher in the corners of these heaters and even for the heater with the rounded corners (figure 4.13e) there is a difference in current density across the heater.

The simulation results for the different heaters can be compared to experimental results. In this case the length of the heating pulse is fixed at $3 \mu$ s and the applied power is increased until bubbles can be detected. Figure 4.18 clearly shows the correspondence between the nucleation sites and points of highest current density. For every heater the bubbles appear on the sites predicted by the simulations. At first it seems strange that bubbles appear in the top right and bottom left corner in figure 4.18b and that in figure 4.18d the bubbles in the top right and bottom left corners are slightly bigger than the bubbles in the other two corners. This can be explained by position of the wide leads (figure 3.5a) that carry the current. The leads are connected to the heater at the top right and bottom left part of heater. This arrangement will result in a increased current density in the top right and bottom left part. The effect was also predicted by simulations.

A closer look at the images of the vapour sheet formed on the heater with rounded corners shown in figure 4.15 reveals that the vapour sheet only covers the part of the heater in between the rounded corners. In the simulation this area corresponds to the 


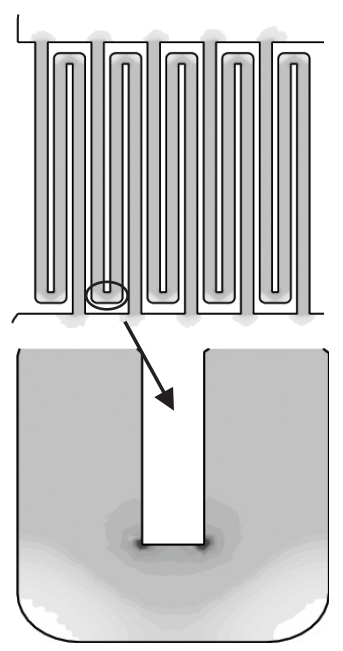

(a)

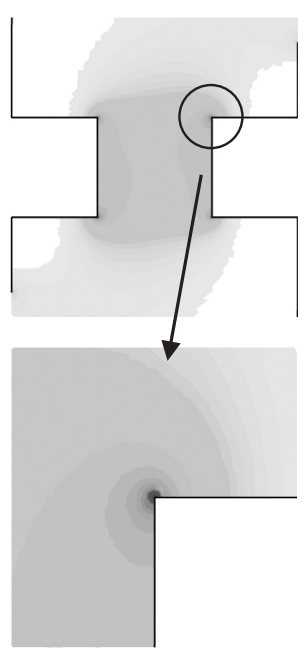

(b)

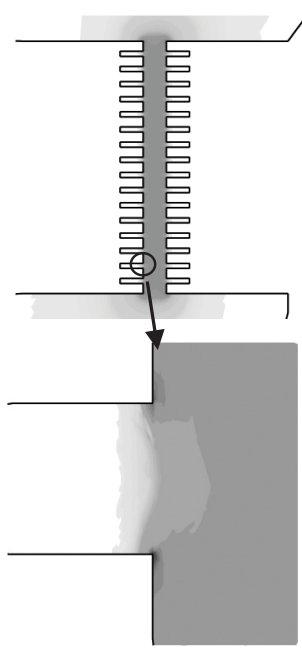

(c)

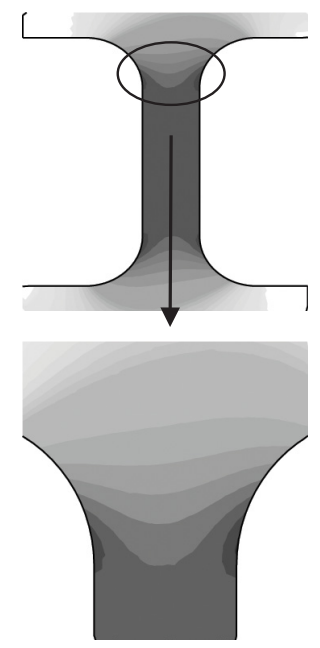

(d)

Figure 4.17: Simulations of the current density for 4 different types of heaters.

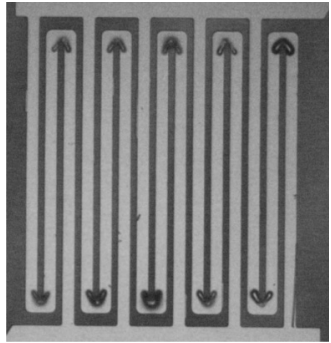

(a)

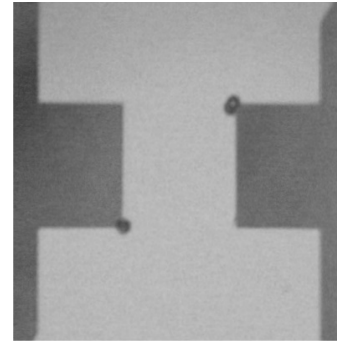

(b)

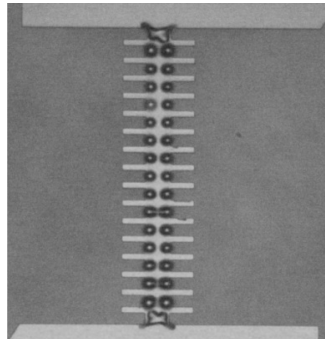

(c)

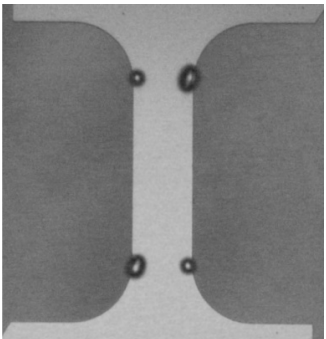

(d)

Figure 4.18: Images of bubble nucleation for 4 different heater geometries. The heaters are identical to the heaters in figure 4.17

part with the highest current densities. The areas above and below those corners are not covered by vapour. In the simulation they have a much lower current density. These areas were considered to be part of the heater, but do not really heat-up the liquid. Therefore the effective heater area is now introduced. The effective heater area is that part of the heater that actually participates in heating the liquid. In this case the area in between the rounded corners. The simulations of the current density can be used to determine the effective heater area.

\subsubsection{Effects of early nucleation}

In the previous subsection we showed that a significant difference in current density in the corners can lead to the nucleation of small bubbles at those corners. This earlier nucleation of bubbles in the corners was also detected by Avedisian et al. [69], but no clear explanation was given about the origin of these bubbles. Bubble growth from the corners can also be caused by growth of small trapped bubbles or nucleation from defects or cavities at those corners. This however is not likely. No bubbles or cavities could be 
detected and although bubbles or cavities smaller than the optical limit of the microscope can not be excluded, it does not explain the nucleation for the sheet with the rounded corners. The fact that the position of leads influences the nucleation strengthens our conclusion that the early nucleation is caused by a difference in current density across the heater.

The early nucleation creates small bubbles, which will generate only a weak pressure pulse. Heating has to be continued to get a vapour sheet across the entire heater, but also the formation of this vapour sheet will be less powerful than an instant vapour sheet formation over the entire heater. The early nucleation will thus be less efficient and therefore undesired. Furthermore the early nucleation makes the determination of the moment of nucleation by heater temperature more difficult. At the high heat-fluxes the moment of nucleation is specified by the change of slope in the temperature versus time curve. A significant change, however, only occurs when a large part of the heater is suddenly covered by the vapour film and a heat transfer changes considerably. The transition from a small bubble in the corners to a full vapour sheet will give rise to a more gradual change in slope. The nucleation of small bubbles will result only in a local temperature change. This effect is too small to show up in the average heater temperature.

A more uniform current density will reduce the error made in the electrical measurements. The temperature gradient across the heater will be smaller and the difference in slope will be more prominent. The measured average temperature will thus be closer to the actual nucleation temperature and the time of nucleation can be determined more accurately. Furthermore the smaller temperature gradient over the heater surface will reduce the chance of an early nucleation.

\subsection{Bubble growth and collapse}

The bubble growth and collapse are more complex than the nucleation. In the first place the bubble growth and collapse depend on the nucleation conditions. A bubble formed by spontaneous nucleation will show a different behaviour than spherical bubble formed during a relatively long and low power heating pulse. Secondly the bubble volume and geometry will depend on the heater size. A vapour sheet formed after spontaneous nucleation will scale with the heater area. A larger heater area will result in a larger vapour volume. The volume of the initial bubble will have a considerable effect on the further development of the bubble. Thirdly the growth and collapse of the bubble will be influenced by the temperature and the pressure of the liquid surrounding the bubble.

The bubble dynamics can be studied by stroboscopic imaging. But in order to conduct a thorough investigation on the dynamics, the bubble volume must be determined. This however, is almost impossible for bubbles generated in the micro bubble actuator, because the height of the bubble is hard to determine. The bubble can only be imaged from the top or bottom. Images from the side are impossible, because the sample is not transparent from the sides. It is possible to use evolution of the light intensity on the bubble interface to calculate the bubble curvature [68], but this method is only useful for the spherical bubbles. The height of the vapour sheet is hard to determine with this method. Therefore only the top view of the bubbles will be considered. 
The bubble growth and collapse can be divided in several stages. The first stage or growth stage starts almost immediately after the nucleation and in this stage the bubble will quickly expand. The second stage or intermediate stage is characterized by alternating bubble growth and collapse. The duration of this stage depends on the heating conditions and under some conditions this stage is even skipped. The third and last stage is the one where the final collapse takes place. This stage ends as the bubble has completely disappeared. In all the stages the speed of bubble growth or collapse and the volume of the bubble will depend on the pulse length, heating power, heater geometry and temperature distribution of the surrounding liquid. The intermediate and final stage will also depend on the applied pressure inside the cavity.

\subsubsection{The growth stage}

The first stage starts directly after bubble nucleation. Figure 4.19 shows the growth stage for a bubble formed during a $200 \mu$ s pulse length and an applied power of $0.49 \mathrm{~W}$. In

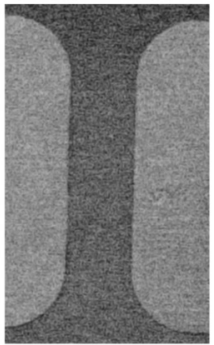

$143 \mu s$

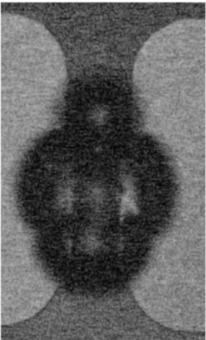

$151 \mu \mathrm{s}$

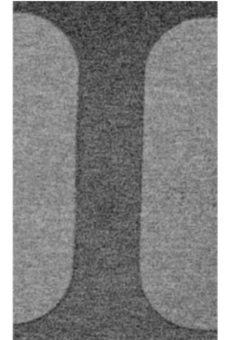

$145 \mu \mathrm{s}$

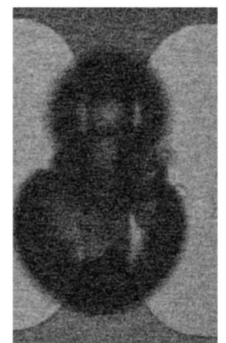

$153 \mu \mathrm{s}$

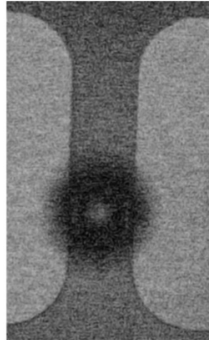

$147 \mu s$

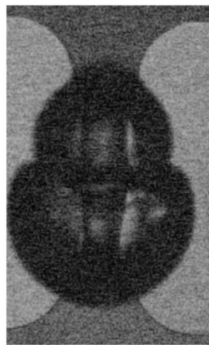

$155 \mu \mathrm{s}$

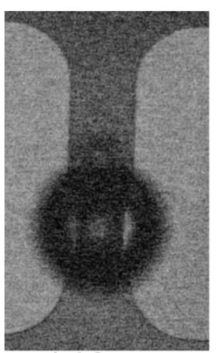

$149 \mu s$

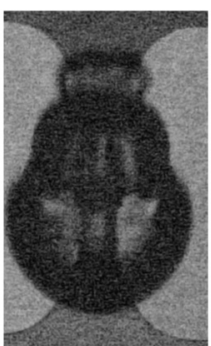

$157 \mu \mathrm{s}$

Figure 4.19: First stage of bubble growth for a $200 \mu$ s pulse length with an applied heating power of $0.49 \mathrm{~W}$

section 4.4.3 it was shown that under these conditions a round bubble grows from a single nucleation site. Here it can be seen that this bubble quickly expands within $10 \mu$ s after the nucleation due to a much higher pressure of the vapour inside the bubble and the presence of superheated liquid. During this time several other bubbles will nucleate on the heater and will also start to grow. These new bubbles can be formed because the heater pulse is still on and the heater temperature will thus be around the nucleation temperature. Depending on the applied heating power the temperature of the heater can increase even further. A larger part of the heater will reach the nucleation temperature and the chance of nucleation increases due to the increase in time and a larger superheated area. The 
newly formed bubbles will coalesce with the bubbles present on the heater. Within 5 to $7 \mu \mathrm{s}$ a large part of the heater is covered with bubbles. The area covered by bubbles in the last three images of figure $4.19(153-157 \mu \mathrm{s})$ differs only slightly. This shows that the expansion of the bubble, at least in the direction parallel to the surface, slows down considerably. The expansion slows down because the pressure inside bubble will decrease with expansion. Further more the bubble interface comes in contact with liquid that is not superheated. Vapour starts to condensate at the top of the bubble, while heating and evaporation still takes place at the heater surface. This will be the start of phase two.

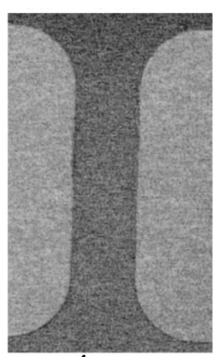

$1 \mu \mathrm{s}$

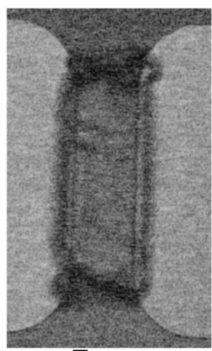

$5 \mu \mathrm{s}$

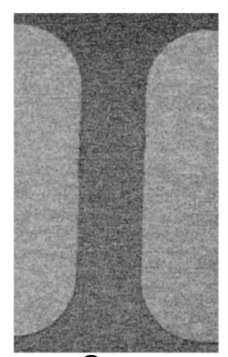

$2 \mu \mathrm{s}$

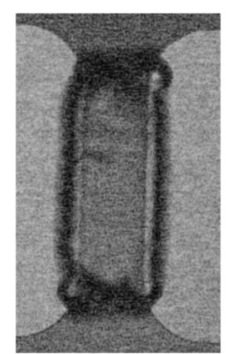

$6 \mu \mathrm{s}$

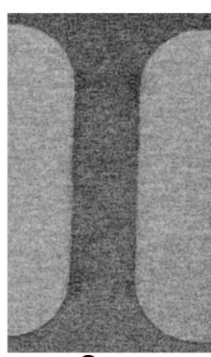

$3 \mu \mathrm{s}$

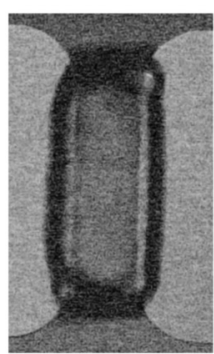

$7 \mu \mathrm{s}$

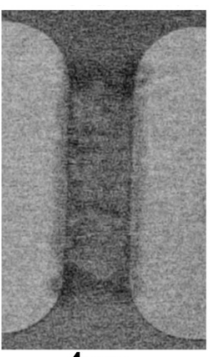

$4 \mu s$

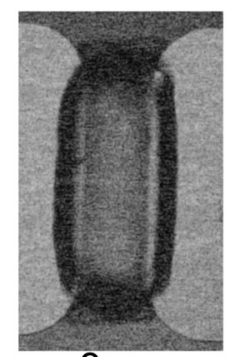

$8 \mu \mathrm{s}$

Figure 4.20: First stage of bubble growth for a $10 \mu$ s pulse at an applied heating power of $3.7 \mathrm{~W}$

For a shorter pulse length and higher applied powers, images of the first stage are shown in figure 4.20. In this case the pulse length is $10 \mu \mathrm{s}$ and the applied heating power $3.7 \mathrm{~W}$. Around $3 \mu \mathrm{s}$ the vapour sheet is formed and covers a large part of the heater surface. The area of the heater and substrate covered by a bubble only slightly increases in the next microseconds. The interface of the bubble is blurry at the moment of nucleation, but becomes sharper with time. The edge becomes darker and in the last two images, taken at 7 and $8 \mu$ s after the start of the heating pulse, the bubble becomes more spherical. At the top and bottom part of the heater a thin vapour layer can also be distinguished. Both ends of the heater part are covered by an expanding thin vapour layer. The centre of the heater is covered by a bubble, which slightly increases in the direction parallel to the heater surface and becomes more rounded as time increases. In the last image taken at $8 \mu$ s the spherical part of the bubble already seems to decrease in size. This is an indication that a next stage in the growth and collapse process is reached.

The exact bubble height and bubble geometry are difficult determine from these images. The difference between bubble growth with long, low power heating pulses and with short, high power heating pulses nevertheless, is evident. At low powers a few rounded 
bubbles grow relatively slowly in a few microseconds. A few microseconds after the first bubble is generated more bubbles nucleate at other parts of the heater as it increases in temperature. The first stage of the growth and collapse process takes about $10 \mu \mathrm{s}$. The bubble radius at the end of the stage is roughly estimated at $100 \mu \mathrm{m}$. This will result in a growth speed of $10 \mathrm{~m} / \mathrm{s}$ and is identical to the growth speed determined in section 4.4.4. At high heating power a vapour sheet covers the heater almost instantly. This vapour sheet hardly grows in the direction parallel to the heater surface and becomes more rounded as the time increases. The first stage at high heating powers takes less than $4 \mu \mathrm{s}$. The height of the vapour sheet is difficult to determine. During the first stage of growth the speed is determined by the pressure difference across the bubble interface and the cooling effect can be neglected. The initial growth speed can be approximated using equation (2.25). If a nucleation temperature of $185^{\circ} \mathrm{C}$ is used the initial pressure will be close to 20 bar. This will result in growth speed of $40 \mathrm{~m} / \mathrm{s}$. This growth speed will however only be reached during the first part of the growth before the bubbles coalesce and the vapour sheet is formed. The length of this period can not be determined from the images shown here. After the coalescence the vapour sheet will grow with a lower speed until a maximum height is reached. Unfortunately it is not possible to calculated this height and therefore also the growth speed from the images shown here.

The size and geometry of the bubble highly dependent on the temperature profile of the liquid at the moment of nucleation. For low powers and long heating pulses the volume of superheated liquid is much larger than for short intensive pulses. The bubble can grow to a larger volume before it comes into contact with sub cooled liquid. Figure 2.12a has shown that at low heating power the temperature profile is more rounded than at high powers (figure 2.6b). This will result in more rounded bubbles during the first period of growth. As the time increases the influence of surface tension will result in more rounded bubbles for both regimes. The pressure inside an initial bubble generated with a short high power heating pulse will be higher than for a long low power heating pulse. This will result in a much higher growth speed just after nucleation for the bubble generated with high heat-fluxes. The faster growth combined with a smaller bubble volume results in a much shorter growth time than for the relatively low power heating pulses.

\subsubsection{The intermediate stage}

After the first stage, in which the initial bubble grows, the bubble starts to decrease in volume. The pressure inside the bubble has decreased considerably and can even become lower than the ambient pressure. The difference in pressure inside and outside the bubble determines the speed of the collapse. Furthermore a part of the bubble interface will be in contact with cold liquid and vapour will start to condensate at the interface. The amount of vapour will decrease and this will enhance the bubble collapse.

Figure 4.21 shows the collapse and re-growth during a $200 \mu$ s heating pulse of $0.49 \mathrm{~W}$. The upper row shows the images taken during the collapse. In $6 \mu$ s the bubble decreases considerably in size. During this decrease the pressure inside the bubble will start to rise again. Between $165 \mu \mathrm{s}$ and $167 \mu \mathrm{s}$ the minimum size is reached and the bubble starts to grow again. The average speed of collapse was estimated at $17 \mathrm{~m} / \mathrm{s}$.

The growth is induced by two mechanisms. In the first place the pressure inside the 


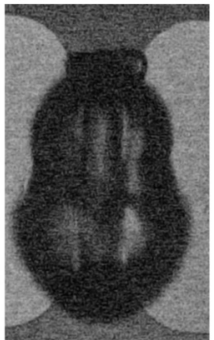

$159 \mu \mathrm{s}$

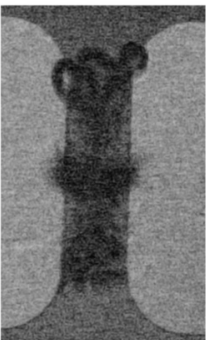

$167 \mu \mathrm{s}$

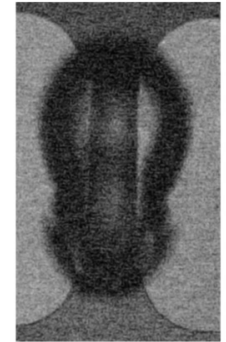

$161 \mu \mathrm{s}$

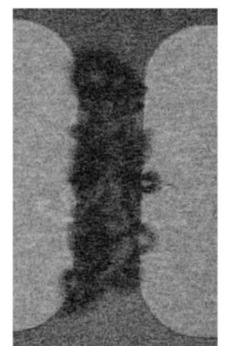

$169 \mu \mathrm{s}$

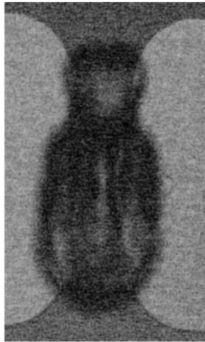

$163 \mu \mathrm{s}$

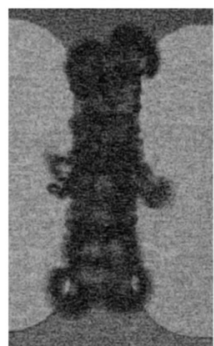

$171 \mu \mathrm{s}$

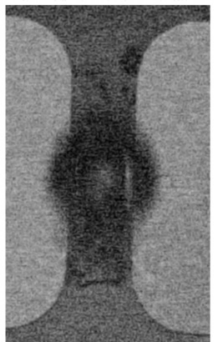

$165 \mu \mathrm{s}$

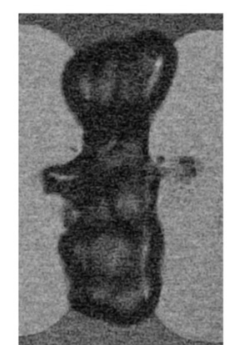

$173 \mu \mathrm{s}$

Figure 4.21: Second stage of bubble growth and collapse for a $200 \mu$ s pulse length with a heating power of $0.49 \mathrm{~W}$

bubble increases considerably during the collapse and can reach values well above the ambient pressure. This pressure difference will result in bubble growth. Secondly the heater surface is still at temperatures above the normal boiling point and the collapsing bubble will result in a flow of relatively cold liquid to the heater surface. The moment this cold liquid gets into contact with or comes close to the heater surface it will be heated and can evaporate. New vapour is formed and the bubble will start to grow again. This re-growth can be seen in the images of the bottom row of figure 4.21 . The fact that the rounded bubble in the middle has completely disappeared and a large number of smaller bubbles grow from the heater, suggest that in this case the re-growth is mainly caused by newly formed vapour bubbles on the hot heater surface.

The intermediate stage of bubble dynamics is also studied for short and more intensive heating pulses. Figure 4.22 shows the collapse and regrowth for a $10 \mu$ s pulse at a power of $3.7 \mathrm{~W}$. The spherical bubble above the vapour sheet decreases in size between $9 \mu$ s and $13 \mu \mathrm{s}$. The average collapse speed is difficult to determine, because the height of the bubble is unknown and the bubble is not spherical. If however a bubble radius of $75 \mu \mathrm{m}$ is used the collapse speed is estimated at $18 \mathrm{~m} / \mathrm{s}$. In the last three images on the bottom row vapour is ejected to the sides at the centre of the heater. This vapour ejection can occur due to high pressures after a fast and non-symmetrical bubble collapse [102]. During the entire sequence a vapour sheet seems to be present on the heater surface. This vapour sheet decreases in size as the bubble on top decrease in size, but after $14 \mu \mathrm{s}$ it starts to grow again. The behaviour of the bubble collapse and re-growth for high power heating powers are more or less similar to the behaviour at low power. The collapse, however, occurs faster and this can result in the ejection of vapour jets. There is also one other important issue that has to be brought to the attention. The pulse length for the sequence 


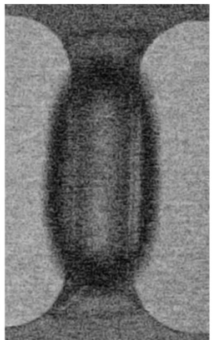

$9 \mu \mathrm{s}$

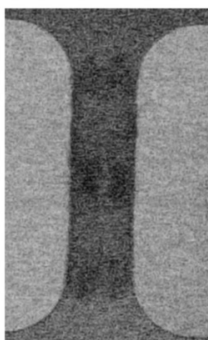

$13 \mu \mathrm{s}$

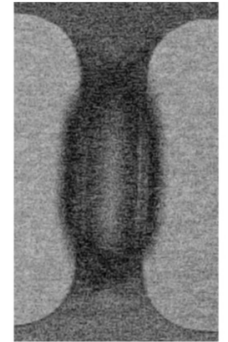

$10 \mu \mathrm{s}$

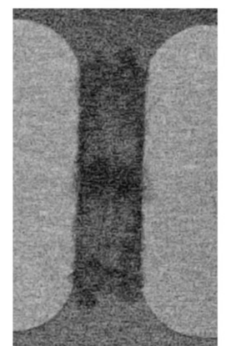

$14 \mu \mathrm{s}$

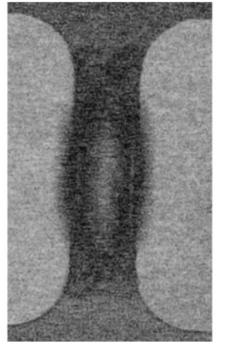

$11 \mu \mathrm{s}$

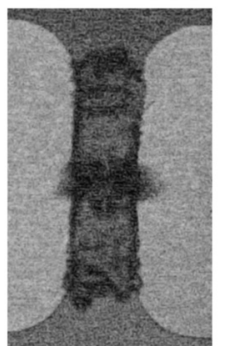

$15 \mu \mathrm{s}$

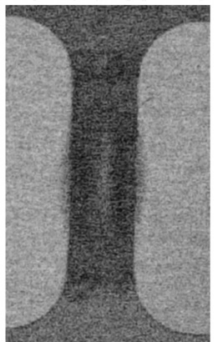

$12 \mu \mathrm{s}$

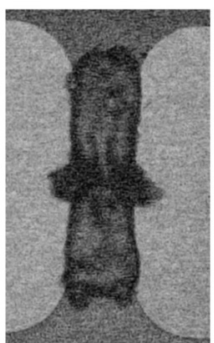

$16 \mu \mathrm{s}$

Figure 4.22: Second stage of bubble growth and collapse for a $10 \mu$ s pulse length with a heating power of $3.7 \mathrm{~W}$

shown in figure 4.22 was $10 \mu \mathrm{s}$, which corresponds to the second image on the top row. For the rest of the images in the sequence the heating was thus turned off and no extra heat was added to the system. The heater quickly starts to cool down. Still the bubble can increase in size between $13 \mu \mathrm{s}$ and $16 \mu \mathrm{s}$, because the heater and the liquid close to the heater will have an elevated temperature.

The moment of bubble collapse and re-growth can also be determined from the temperature versus time curve. Figure 4.23 shows the heater temperature during a $50 \mu$ s pulse. The drops in temperature indicated by a,b and c are the result of an increase in energy transfer from the heater. It was already mentioned that during the bubble collapse cold liquid starts to flow to the heater and that this cold liquid will evaporate at the moment it comes into contact with the heater. Energy is extracted from the heater and this will result in a temperature drop of the heater surface. The images in figure 4.24 are taken around the time of a,b and c respectively. For each row the middle picture shows the smallest bubble size and this thus corresponds with the end of a collapse period. The difference in bubble size as well as the temperature drop in figure 4.23 decrease with time.

The moment of collapse among others depends on the applied heating power. Figure $4.25 \mathrm{a}$ shows the time of the first collapse versus the applied heating power. The time at which the bubble collapses for the first time decreases from $168 \mu \mathrm{s}$ at $0.49 \mathrm{~W}$ to $22 \mu \mathrm{s}$ at $2,4 \mathrm{~W}$. The curve shows a resemblance with the nucleation versus power curve shown in figure 4.6. In figure 4.25b the difference in time between the first collapse and the nucleation as well as time between the successive collapses was plotted against the applied power. The time between the moment of nucleation and the first collapse decreases from $20 \mu \mathrm{s}$ at low power to $13 \mu \mathrm{s}$ at relatively high power. The time between the first collapse and the second collapse decreases from $16 \mu \mathrm{s}$ to $9 \mu \mathrm{s}$ for applied powers between $0.49 \mathrm{~W}$ 


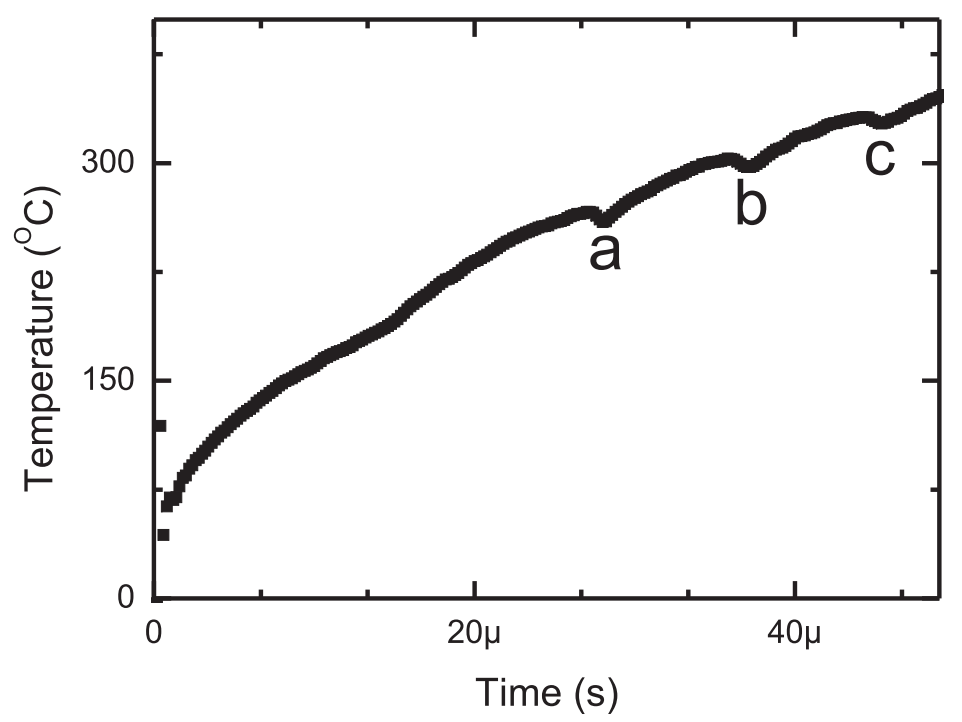

Figure 4.23: The heater temperature during $1.7 \mathrm{~W}$ heating pulse of $50 \mu$ s pulse. Three dips in temperature due to bubble regrowth can be detected.

and $2.4 \mathrm{~W}$. The time between the second collapse and the third collapse changes only a little, from $11 \mu \mathrm{s}$ to $9 \mu \mathrm{s}$. For high powers the time between nucleation and first collapse becomes constant around $13 \mu \mathrm{s}$ and the time between the successive collapses becomes constant around $9 \mu \mathrm{s}$. The same behaviour and nearly identical values for the difference in time between cavity formation and first time of collapse were found by Varlamov et al. [50]. In this paper only the first collapse was considered and a bubble rebound was only briefly mentioned.

In figure 4.25 only the first three moments of collapse were considered, but the bubble continues the grow and collapse. If the heating is continued in this case the bubble will grow, but with each growth and collapse period the difference in bubble size and temperature drop will be smaller. At some point a steady-state situation will be reached where condensation and evaporation rates are constant. It will depend on the heating power and heat transfer in the system if the bubble volume is constant or that it will grow or collapse. In most cases, however, the heating is turned off after some time. The bubble will start a period of continuous decrease in size and this will be the start of the final stage.

\subsubsection{The final stage}

In the final stage the bubble size will on average decrease in size. Alternating growth and collapse periods, nevertheless, are still possible. In figure 4.26 the last stage for a bubble generated by a $200 \mu$ s pulse of $0.49 \mathrm{~W}$ is shown. The first images show the bubble just after the heating pulse is turned off. The bubble starts to decrease in size almost immediately after the heating pulse is turned off and this decrease continues for about $10 \mu \mathrm{s}$ to $15 \mu \mathrm{s}$. During this decreases a large number of smaller bubbles are released from the large bubble at the centre of the heater and at $211 \mu$ s the bubble at the centre also breaks up in several smaller bubbles. After this division the decrease in bubble size slows 

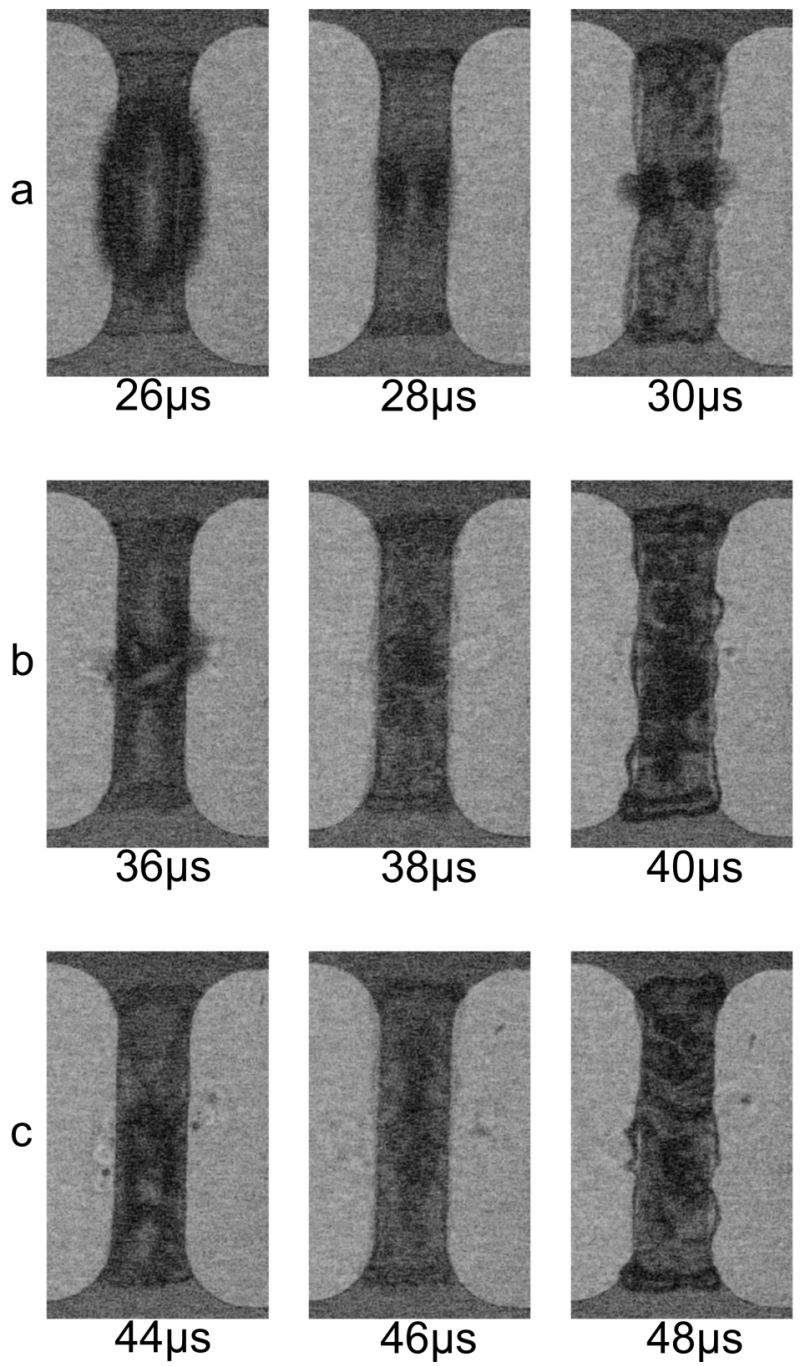

$48 \mu \mathrm{s}$

Figure 4.24: Images of collapse and re-growth for a $50 \mu$ s pulse at an applied power of $1.7 \mathrm{~W}$

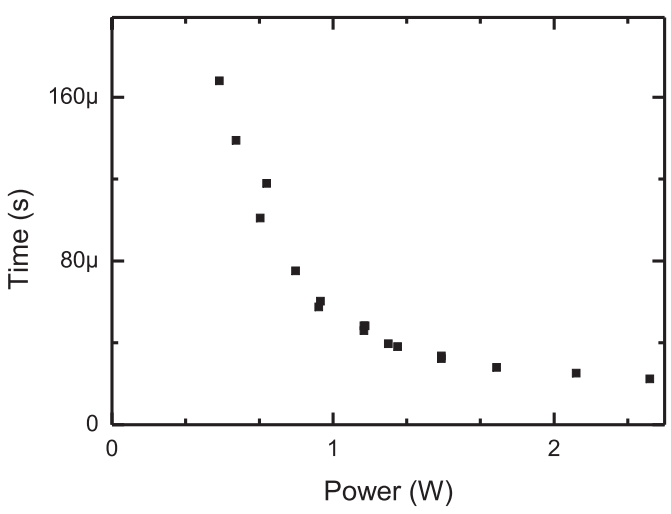

(a) Time of collapse

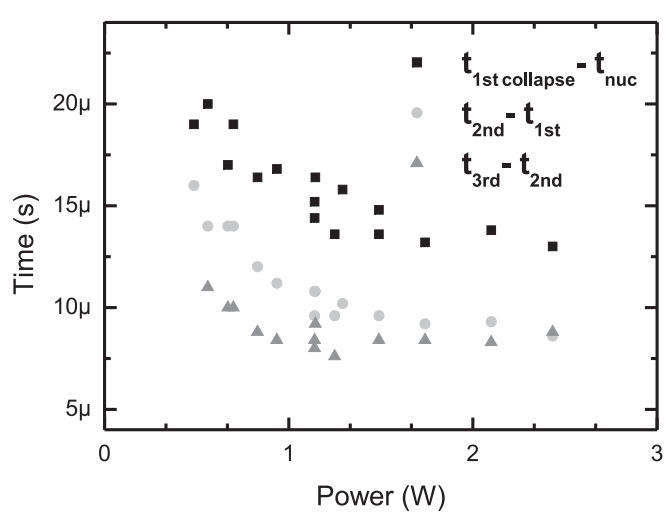

(b) Time difference

Figure 4.25: Time of collapse and the time difference between the collapse times and nucleation as a function of the applied power. 


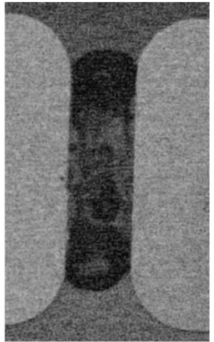

$203 \mu \mathrm{s}$

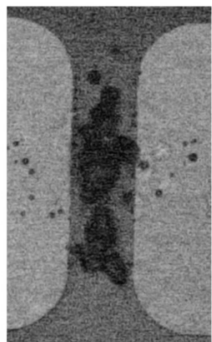

$211 \mu \mathrm{s}$

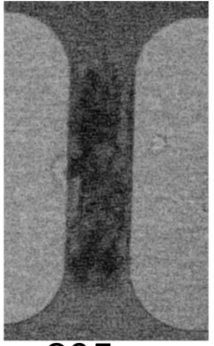

$205 \mu \mathrm{s}$

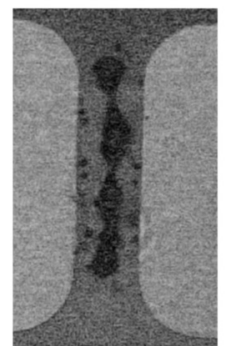

$215 \mu \mathrm{s}$

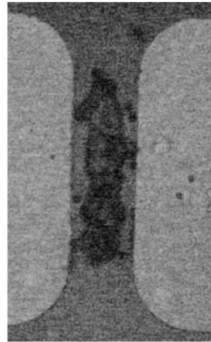

$207 \mu \mathrm{s}$

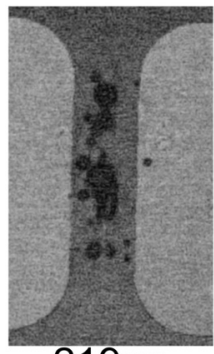

$219 \mu \mathrm{s}$

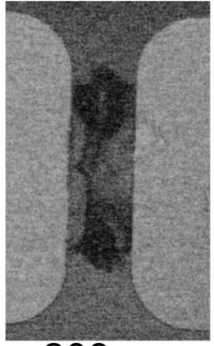

$209 \mu \mathrm{s}$

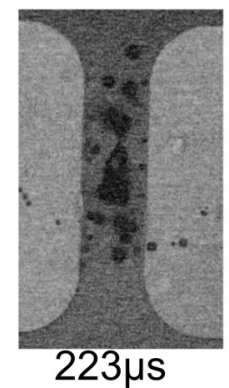

Figure 4.26: Third stage of the bubble for 200 pulse length with heating power of $0.49 W$.

down considerably.

For a $10 \mu \mathrm{s}$ pulse at $3.7 \mathrm{~W}$ the final stage starts at $21 \mu \mathrm{s}$ after the start of the heating pulse. The power is thus already off for $11 \mu$ s before the final collapse takes place. Figure 4.27 shows that a large bubble covering the active part of the heater decreases to a few small bubbles within $10 \mu \mathrm{s}$. These small bubbles, however, disappear only slowly and at $50 \mu$ s they are still present. The decrease in size is not continuous, after $23 \mu$ s a short period of bubble growth can be seen. The decrease in bubble size continues again after $25 \mu$ s. This alternating growth and collapse is caused by the same factors as in the intermediate stage, but in this case no heat is added to the system any more and the bubble will on average decrease in size.

At both low and high heating power the bubble collapse is relatively fast during the first part of the final stage. The large bubble reduces to a number of small bubbles within $10 \mu \mathrm{s}$ to $15 \mu \mathrm{s}$. These smaller bubbles, however, take much longer to collapse. One of the reasons of this slow collapse is the elevated liquid temperature around and on the heater. The moment the heater is turned off the heater itself and the liquid surrounding the heater will cool down to a temperatures close to the initial temperature within a number of microseconds. During this period the temperature can be high enough to sustain vapour bubbles. The time it takes to reach a temperature in which vapour bubbles can not survive depends on the pulse time and the applied power. In section 2.2 it was already shown that for low heating power a relatively large volume is heated and the decrease to the initial temperature will take longer. The time before all the bubbles have disappeared will take more time than for high heating powers.

The bubbles probably contain other gasses beside the vapour. These residual gasses can only leave the bubble by diffusion. This will take much longer than condensation of 


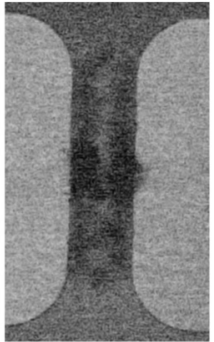

$21 \mu \mathrm{s}$

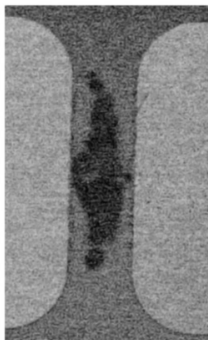

$25 \mu s$

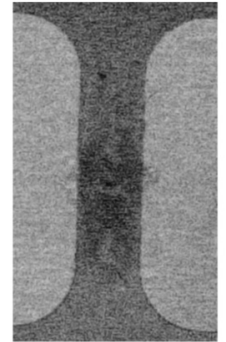

$22 \mu \mathrm{s}$

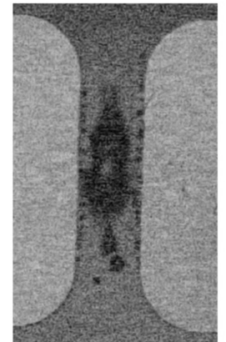

$29 \mu s$

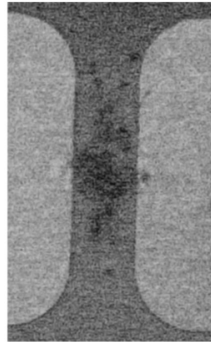

$23 \mu \mathrm{s}$

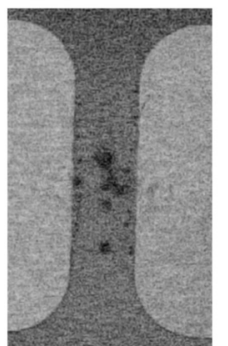

$32 \mu \mathrm{s}$

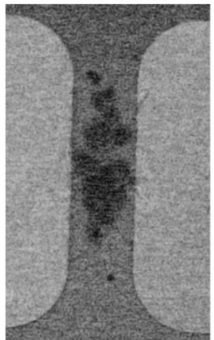

$24 \mu s$

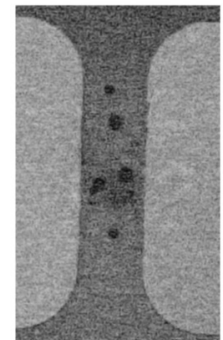

$50 \mu s$

Figure 4.27: Third stage of the bubble for 10 pulse length with heating power of $3.7 \mathrm{~W}$.

vapour. The small bubbles in the last image of figure 4.27 will probably contain mostly gas and the small droplets ejected from heater in the last image of figure 4.26 are probably also gas bubbles. Due to the presence of an unknown amount of residual gasses it is difficult to investigate the collapse speed of the vapour bubbles and study the final stage of collapse more adequately.

\subsubsection{The influence of the cavity pressure}

The pressure inside the cavity will have a large effect on the bubble dynamics. For short pulses with a high heating power the pressure inside the cavity will have a negligible influence on the nucleation. The limit of superheat will change a little bit, but this will have almost no effect on the time of spontaneous nucleation. As the pulse length increases the influence of pressure on nucleation should increase, but because the micro bubble actuator is intended to work with microsecond heating pulses, this was not investigated.

The first stage of bubble dynamics will show some dependence on cavity pressure. If we assume the bubble to be in mechanical equilibrium the volume of the bubble (in this case the vapour sheet) will decrease with the pressure inside the cavity(equation(2.15)). The developed pressure impulse will decrease with an increase in cavity pressure and therefore the initial growth speed given by equation(2.25) and the maximum bubble volume (equation(2.27)) will be lower. The difference in cavity pressure and the pressure inside the initial bubble will, however, always be large and the effect of the cavity pressure on the pressure impulse will be small.

Figure 4.28 shows images of the bubble at $3 \mu \mathrm{s}$ and at $5 \mu \mathrm{s}$ for a $3 \mu \mathrm{s}$ pulse at $3.0 \mathrm{~W}$. For the top row the pressure inside the cavity is equal to the ambient pressure. At $3 \mu \mathrm{s}$ the heater is covered by a vapour sheet and around $5 \mu$ s the bubble has become spherical 


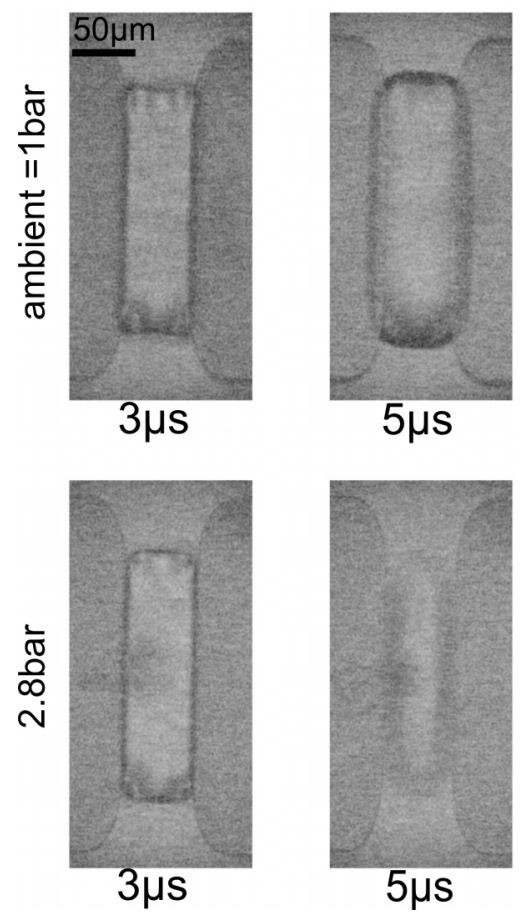

Figure 4.28: First stage of bubble dynamics for a $3 \mu$ s pulse at $3.0 \mathrm{~W}$ at ambient pressure inside the cavity and at a pressure difference of 1.8 bar between the cavity and the outside pressure

and reaches its maximum bubble size. The bottom row shows images taken at the same time, but the pressure inside the cavity is increased. The difference in pressure between the cavity and the outside world is $1.8 \mathrm{bar}$. The absolute pressure inside the cavity will thus be $2.8 \mathrm{bar}$. The size and geometry of the vapour sheet at $3 \mu \mathrm{s}$ are almost identical to the ones at ambient pressure. The height of the vapour sheet will be slightly smaller due to the increased pressure, but this can not be determined from the images. At $5 \mu$ s the image taken at a pressure difference of 1.8 bar is completely different from the one taken at ambient pressure. At a difference of 1.8 bar the outline of the bubble is vague and much smaller than the outline at ambient pressure. The bubble has already past the first stage and is collapsing.

Figure 4.29 shows the collapse of a bubble at ambient pressure and at a pressure difference of $1.8 \mathrm{bar}$ for a $3 \mu \mathrm{s}$ pulse at $3.0 \mathrm{~W}$. At ambient pressure the total collapse takes place between $7 \mu \mathrm{s}$ and $12 \mu \mathrm{s}$ and takes about $5 \mu \mathrm{s}$. The speed of the bubble collapse was estimated from the images at $15 \mathrm{~m} / \mathrm{s}$. At a difference of 1.8 bar the bubble collapse starts several microseconds earlier and takes about $3 \mu \mathrm{s}$. The collapse speed is estimate at $20 \mathrm{~m} / \mathrm{s}$. From figure 4.28 it can be concluded that the collapse already starts between $3 \mu$ s and $5 \mu \mathrm{s}$. The collapse slows down after $7 \mu \mathrm{s}$. In order to make a better comparison we look at the bubble size during the collapse. The time between bubbles of identical size for ambient pressure and a pressure difference of $1.8 \mathrm{bar}$ was estimated. A size equal to the bubble size at $5 \mu \mathrm{s}$ for the elevated pressure will at ambient pressure probably be found between 7 and $9 \mu$. Furthermore a size equal to the bubble size at $12 \mu$ s and ambient pressure can be found around $7 \mu$ s for the pressure difference of $1.8 \mathrm{bar}$. The collapse of 

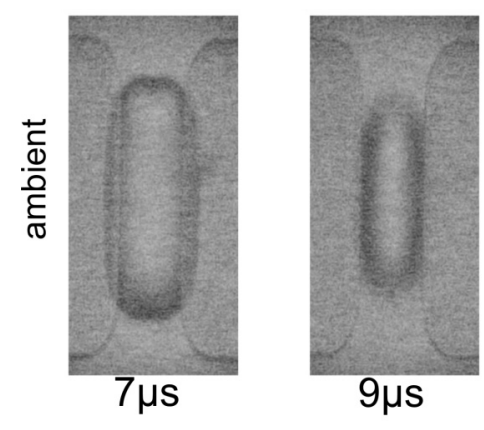

$9 \mu \mathrm{s}$

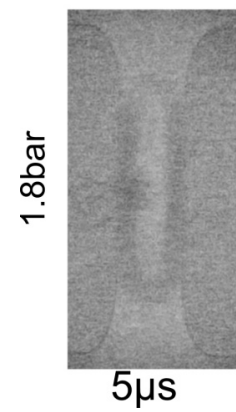

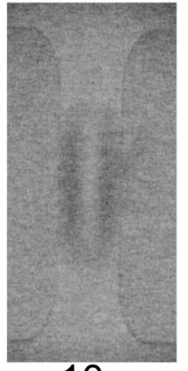

$10 \mu \mathrm{s}$

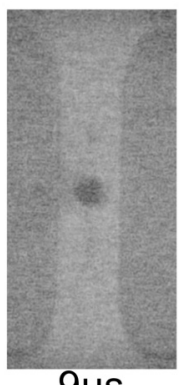

$9 \mu \mathrm{s}$

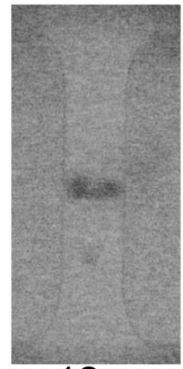

$12 \mu s$

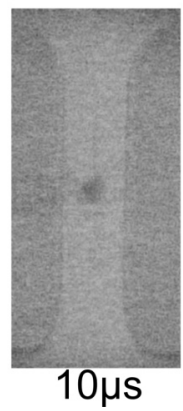

Figure 4.29: Bubble collapse for a $3 \mu \mathrm{s}$ pulse at $3.0 \mathrm{~W}$ at ambient pressure inside the cavity and at a pressure difference of 1.8 bar between the cavity and the outside pressure

a similar bubble takes about $4 \mu \mathrm{s}$ at ambient pressure and only $2 \mu$ s at a 1.8 bar pressure difference. The collapse speed at elevated pressure is thus twice as high. The accuracy of these measurements however is rather low and the images don't show enough detail to do a good analysis on the exact speed and size of the bubbles. Equation (2.32) gives the collapse time of a cavitation bubble. For a bubble with $70 \mu \mathrm{m}$ radius, which is comparable to the size of the bubble found here, it takes $6 \mu$ s to fully collapse at ambient pressure and $4 \mu \mathrm{s}$ at 1.8 bar pressure difference. The average collapse speed of a spherical bubble with a $70 \mu \mathrm{m}$ radius will thus be $12 \mathrm{~m} / \mathrm{s}$ at ambient pressure and $18 \mathrm{~m} / \mathrm{s}$ at a pressure difference of 1.8 bar. Although the collapse speed will change with time, the bubble geometry is not spherical and the bubble size or volume are difficult to determine. The collapse speed calculated from the images is comparable to the collapse speed expected during bubble cavitation. Experiments done by Hong et al. [65] give values of $10 \mathrm{~m} / \mathrm{s}, 20 \mathrm{~m} / \mathrm{s}$ and $50 \mathrm{~m} / \mathrm{s}$ for the velocity of the bubble interface in different directions during the collapse. The highest speed was measured in the z-direction.

The complete bubble collapse can take much longer. In the previous section the influence of residual gas was already mentioned. For a $3 \mu$ s pulse at $3.0 \mathrm{~W}$ it takes less than $50 \mu \mathrm{s}$ to cool the heater and its surroundings to temperatures below the boiling point of ethanol. Vapour bubbles present in the liquid will collapse within microseconds, because the vapour will quickly condensate at temperatures below the boiling point. If,however, another gas is also present in the bubble, the gas has to dissolve completely in the liquid to get a full bubble collapse. The dissolvation of gas into liquid is a process governed by diffusion. The speed of this process depends parameters, such as initial gas concentration in the liquid and in the bubble, temperature, pressure and initial bubble volume.

An increase in pressure is expected to increase the bubble collapse speed and will thus 


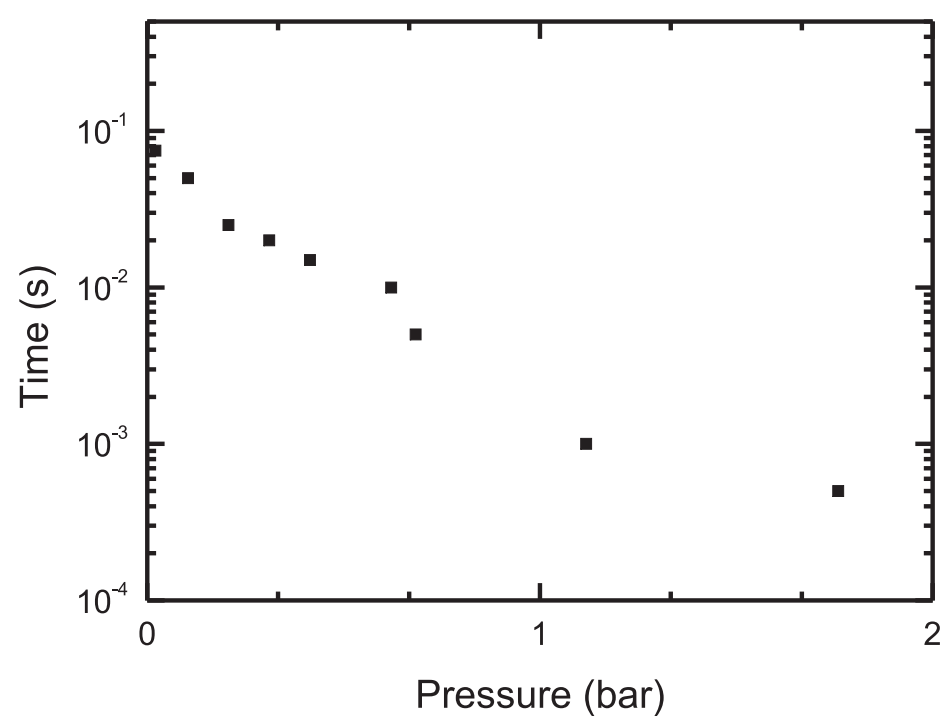

Figure 4.30: Time of complete bubble collapse as a function of the applied pressure difference across the membrane.

shorten the time it takes a gas bubble to collapse. Figure 4.30 shows the time it takes for all the bubbles to collapse completely as a function of pressure. At ambient pressure it takes almost $75 \mathrm{~ms}$ before all bubbles have disappeared. At $1.8 \mathrm{bar}$ this time is reduced to $500 \mu \mathrm{s}$. The time of collapse, as expected, decreases with applied pressure. The time of collapse at ambient pressure as well as at elevated pressure is much longer than it would take a vapour bubble to condensate. The bubbles formed during the measurements most probably contain a certain amount of residual gas. The collapse of gas bubbles in liquids is described by Epstein et al. [103]. The speed of collapse is highly dependent on the concentration of gas in the liquid. For an air bubble with a radius of $10 \mu \mathrm{m}$ in water the duration of the collapse is between $1 \mathrm{~s}$ and 5 s depending on the concentration of air in the water. The solubility of gasses in liquids will depend on the particular gas and the liquid in which the gas must dissolve. The solubility of gasses in different liquids was investigated by Leites et al. [104] and can be used to predict the dissolution for the different gasses in ethanol.

\subsection{Repetition frequency}

For all the measurements discussed in the previous sections the time between the individual pulse was relatively long. In most cases repetition frequency was kept below $10 \mathrm{~Hz}$. This ensured that the generated bubbles had condensed or dissolved and the liquid was again at ambient temperature. The goal of the project, however, is to produce a fast actuator working at several $\mathrm{kHz}$. Increasing the repetition frequency to these frequencies will have an effect on the temperature distribution in the system, on the bubble generation and on the bubble growth and collapse. 


\subsubsection{Effects on the temperature}

In section 2.2 the effect of the repetition frequency on the temperature distribution in the system was already discussed. An increase in repetition frequency results in a decrease in cooling time. The heater temperature at the start as well as the end of the heating pulse will increase with the number of pulses until a steady state situation is reached. Figure 4.31 shows the result of a temperature measurement for a $0.65 \mathrm{~W}$ heating pulse

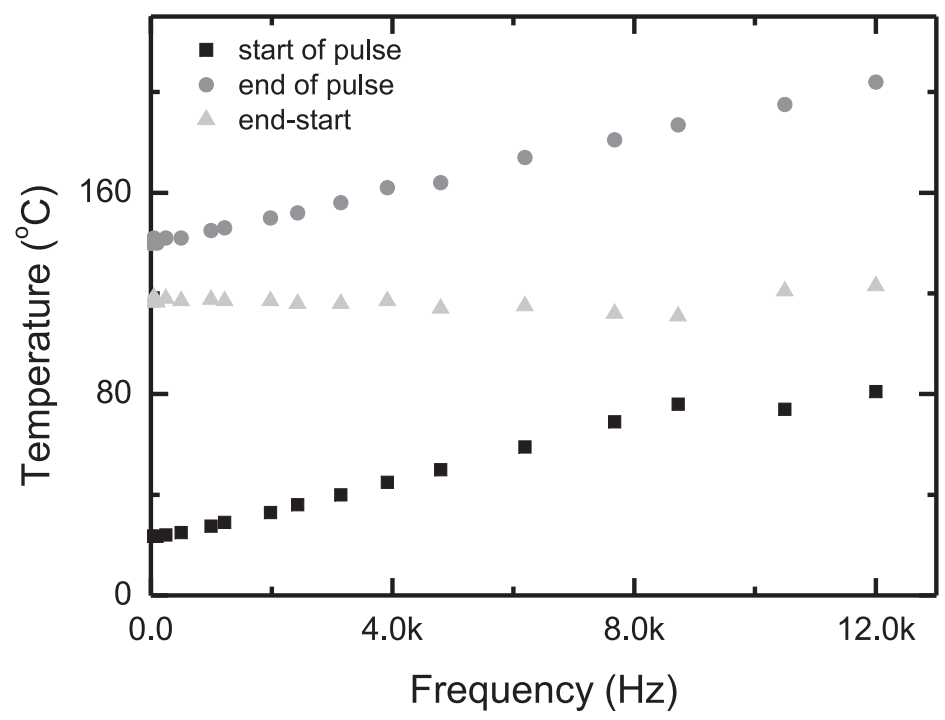

Figure 4.31: Heater temperature at the start and at the end of the heating pulses and the temperature difference between the start and the end of a single as a function of repetition frequency for a $0.65 \mathrm{~W}$ heating pulse of $3 \mu \mathrm{s}$

of $3 \mu$ s with a repetition frequency between $1 \mathrm{~Hz}$ and $12 \mathrm{kHz}$. The heater temperature at the end and just before the start of a heating pulse were measured. From this the temperature difference between the start and the end of heating pulse was calculated. The temperature at the start of the heating pulse increases linearly from $23^{\circ} \mathrm{C}$ to $81^{\circ} \mathrm{C}$ and the temperature at the end increases from $140^{\circ} \mathrm{C}$ to $204^{\circ} \mathrm{C}$. The temperature difference between the start of the heating pulse and the end of the heating pulse stays nearly constant at $117^{\circ} \mathrm{C}$. The temperature difference is thus independent of frequency. For a single pulse the temperature difference between the start and the end of the heating pulse can be described by equation (2.8). At higher frequencies the temperature difference is also constant and equation (2.8) can thus still be used to calculate the heater temperature, but one has to now the temperature at the start of the heating pulse.

The difference in heater temperature before and after the heating pulse depends on the heating power and the pulse time. Both parameters will also have an influence on the temperature at the start of the heating pulse. Figure $4.32 \mathrm{a}$ shows the difference between the temperature at the start of the heating pulse and the ambient temperature as a function of frequency for three different pulse lengths at heating power of $0.43 \mathrm{~W}$. The temperature difference increases linear with frequency. The slope of the curve depends on the pulse length. The maximum temperature difference is $91^{\circ} \mathrm{C}$ for a pulse length of $10 \mu \mathrm{s}$ and a repetition frequency of $10 \mathrm{kHz}$. The temperature difference is only $41^{\circ} \mathrm{C}$ for a 
$3 \mu \mathrm{s}$ pulse at the same repetition frequency. Figure $4.32 \mathrm{~b}$ shows the result of an identical measurement, but in this case three different heating powers were used and the pulse length was constant at $3 \mu \mathrm{s}$. At low frequency the temperature also increases linear with frequency. At higher frequencies, however, a change in slope occurs for the higher heating pulses. This slope change occurs around $9 \mathrm{kHz}$ and at $2,5 \mathrm{kHz}$ for an applied heating power of $0.65 \mathrm{~W}$ and $1.0 \mathrm{~W}$ respectively.

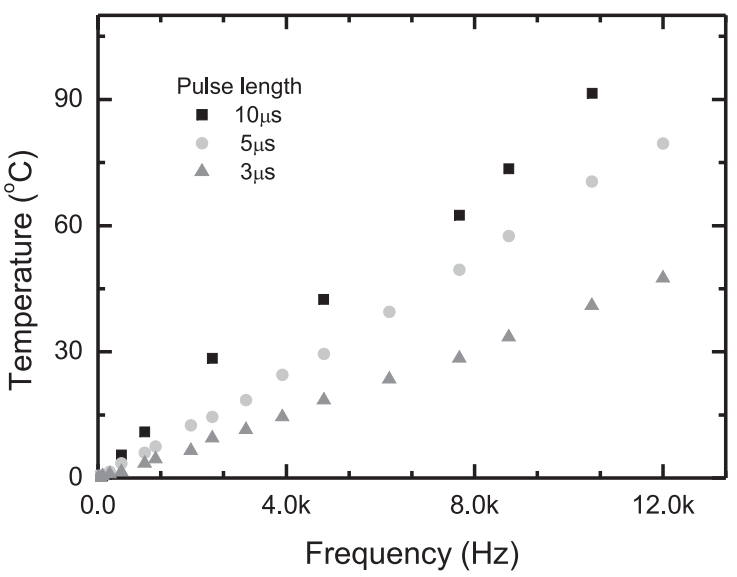

(a) Different pulse lengths

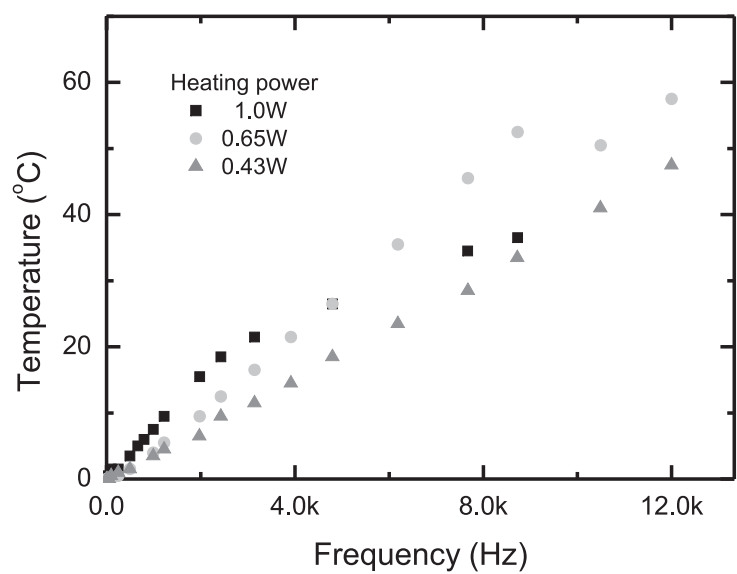

(b) Different heating powers

Figure 4.32: Difference between the heater temperature at the start of the heating pulse and ambient temperature as a function of repetition frequency

The slope change occurs due to the formation of bubbles. This was confirmed by optical observation. In this case instead of stroboscopic imaging, a camera with normal continuous illumination was used. This method will only show events that are present for a relatively long time. Images taken at low repetition frequencies don't show any bubbles. Bubble nucleation either does not take place or generated bubbles collapse so fast that they don't show up in the images. The images, taken at frequencies just above the slope change, show permanent stable bubbles. This indicates that the bubbles are present for a relatively long time, at least much longer than the collapse of a single bubble under normal conditions. If the frequency is increased further, parts of the bubbles start to detach from the heater and a train of rising bubbles is formed.

Each pulse introduces a fixed amount of energy into the system. This energy per pulse $E$ is given by equation (4.3). After a number of pulses a steady state situation is reached and the average power $\left(P_{\text {avg }}\right)$ is given by

$$
P_{\text {avg }}=E * f
$$

where $f$ is the repetition frequency.

In figure 4.33 the results of figure 4.32 are shown as a function of average power. At low average power the difference in temperature at the start of the heating pulse and the ambient temperature is independent of the applied heating pulse or pulse length. Of course both parameters do effect the average power itself. The temperature at the start of the heating pulse increases linearly with the average power. At higher average powers 
a change of slope occurs. The average power at which this slope change occurs depends on pulse length and applied heating power. At a constant heating power a chance of slope only occurs for the $10 \mu \mathrm{s}$ at a average power of $10 \mathrm{~mW}$. At a constant pulse length of $3 \mu \mathrm{s}$ the slope change occurs at $18 \mathrm{~mW}$ and $7 \mathrm{~mW}$ for a $0.65 \mathrm{~W}$ and $1.0 \mathrm{~W}$ respectively.

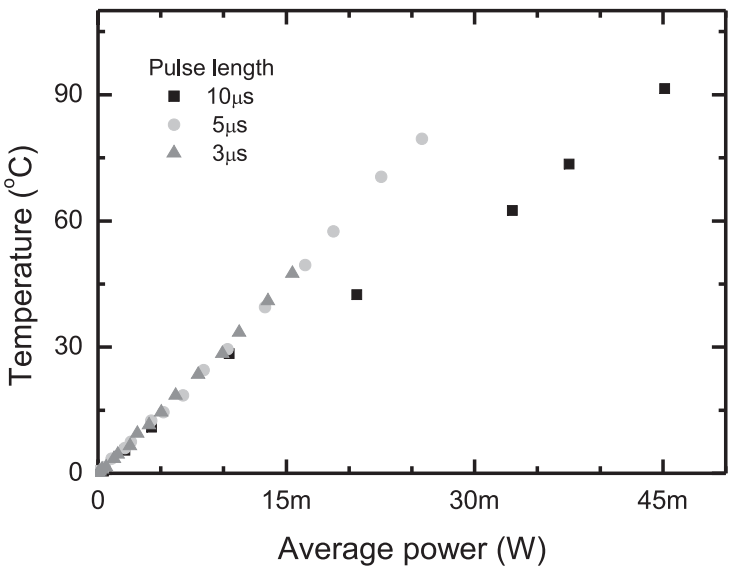

(a) Different pulse lengths

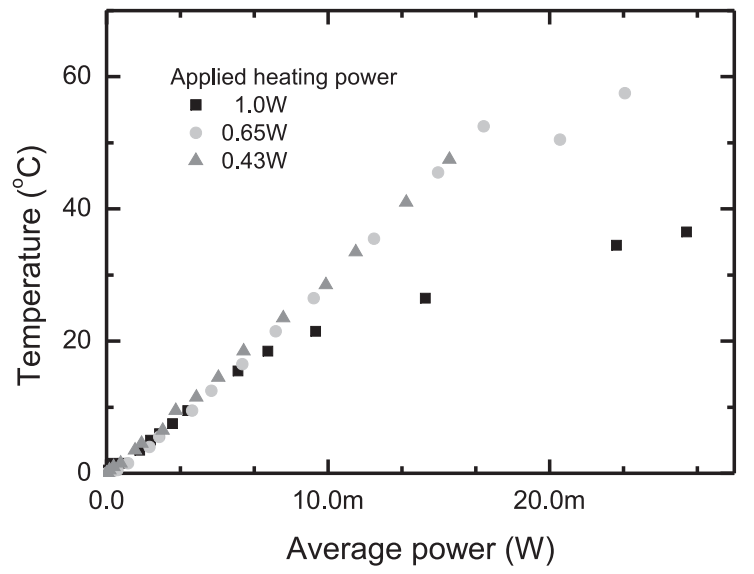

(b) Different heating powers

Figure 4.33: Temperature difference between the ambient temperature and the heater temperature at the start of a heating pulse as a function of average power for a $3 \mu$ s heating pulse.

At relatively low average heating powers the difference in temperature at the start of the heating pulse and the ambient temperature $\left(T_{d i f}\right)$ can thus be estimated using

$$
T_{d i f}=\frac{P_{a v g}}{h}
$$

where $h$ is a sort of heat-transfer coefficient. A linear fit to the results found in figure $4.33 \mathrm{a}$ and $4.33 \mathrm{~b}$ results in a value of $3.5 \times 10^{-4} \mathrm{~W} / \mathrm{K}$ for $h$. In this case only the results before a slope change were used in the fit.

The generation of bubbles and the bubble collapse leads to change in heat-transfer. In this case the heat-transfer is not only determined by heat-diffusion, but also by convection. This change in heat-transfer gives rise to the slope change in the temperature versus frequency and average power plots. The average power at which this slope chance occurs increases with a decreasing applied heating power and with a decreasing pulse length. More generally one can conclude that the frequency and average heating power at which the slope change takes place, increases with a decrease in energy input per pulse. The slope and thus the heat-transfer coefficient always changes to a lower value. The generation and growth and collapse of bubbles improve the heat-transfer through the system.

\subsubsection{Effect on the bubble nucleation}

The heat-transfer in the system is influenced by the formation of bubbles, but on the other hand the formation of bubbles will be influenced by the temperature of the heater 
and the liquid close to the heater. For short pulses, the bubble nucleation takes place at the moment the heater temperature reaches values near the kinetic limit of superheat. For a fixed pulse length and heating power this temperature will be reached at an earlier stage as the repetition frequency increases. Figure 4.34 shows the bubble nucleation time

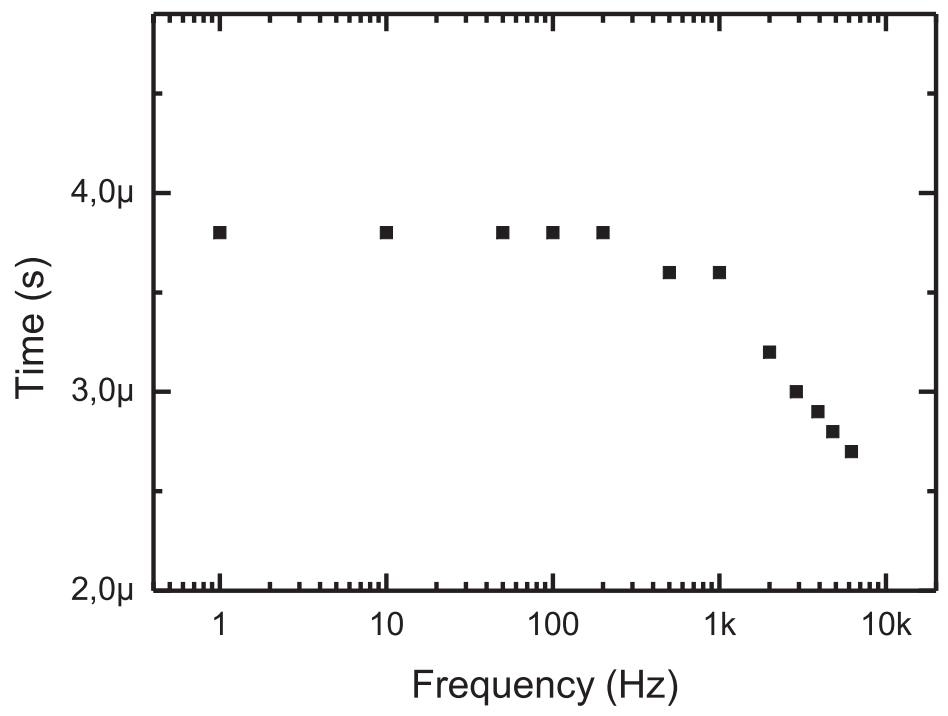

Figure 4.34: Time of bubble nucleation as a function of repetition frequency for a $3 \mu \mathrm{s}$ pulse at a $3.0 \mathrm{~W}$ heating power.

for a $3 \mu$ s pulse with a heating power of $3.0 \mathrm{~W}$ as a function of repetition frequency. Up to a frequency of $250 \mathrm{~Hz}$ the nucleation time is constant at $3,8 \mu \mathrm{s}$ after the start of the heating pulse. The bubble nucleation thus takes place after the end of the heating pulse. Varlamov et al. [72] show that under certain conditions bubble generation is possible after a heating pulse has stopped, because the temperature of liquid continues to increase for a short time. Also the simulations in section 2.2 show that the temperature of the liquid can still increase after the heating pulses has stopped. Above $250 \mathrm{~Hz}$ the nucleation time decreases with frequency and at $6.2 \mathrm{kHz}$ the time of nucleation has decreased to $2.7 \mu \mathrm{s}$.

The temperature at the end of the heating pulse depends on the temperature at the start of the heating pulse, the pulse length and the heating power. By changing these parameters the heater temperature during and at the end of the heating pulse can be controlled. In this case the minimum energy, which is the product of the heating power and pulse length, to create a bubble is determined for a constant pulse length by increasing the applied power until the nucleation temperature is reached at the end of the heating pulse. Bubble nucleation therefore also takes place at the end of the heating pulse. Figure 4.35 shows the minimum energy per pulse needed to get a nucleation event as a function of frequency for a $3 \mu$ s pulse. An increase in frequency increases the temperature at the start of the heating pulse and the difference between this temperature and the nucleation temperature decreases. At higher frequencies a lower heating power is thus already sufficient to create a nucleation event. The identical procedure was used to determine the minimum energy to create a vapour sheet at the end of the heating pulse. At relatively low repetition frequency the minimum energy is almost constant at $10 \mu \mathrm{J}$ for bubble nucleation and $11 \mu \mathrm{J}$ to create a vapour sheet. Above $250 \mathrm{~Hz}$ the minimum energy 


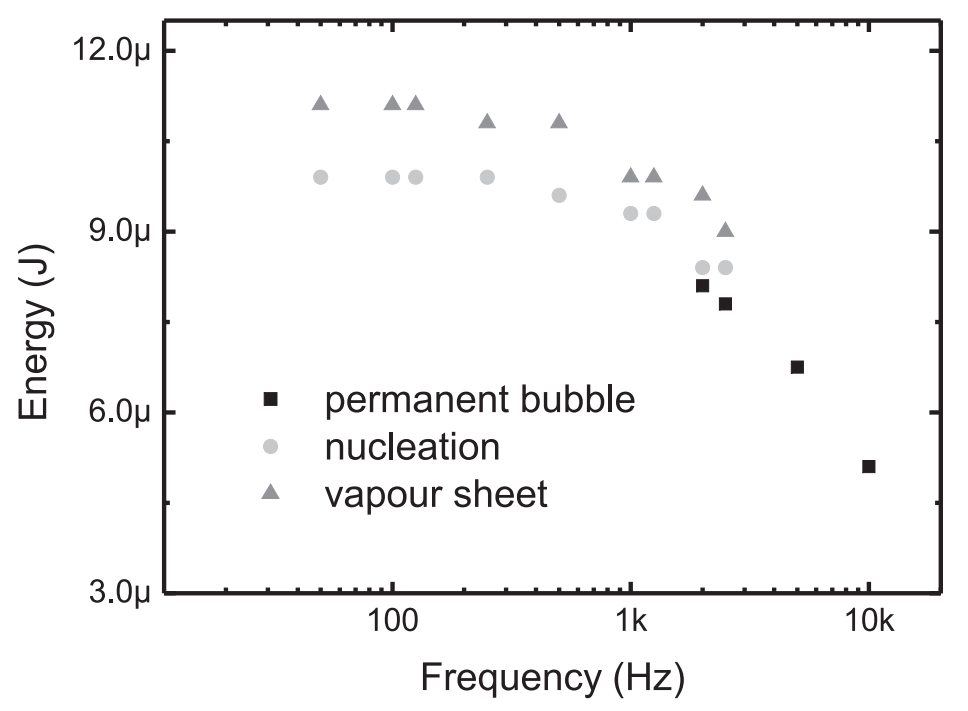

Figure 4.35: Minimum energy per pulse needed to create a nucleation event or vapour sheet for a $3 \mu \mathrm{s}$ pulse length. At higher frequencies the minimum energy to create a permanent bubble is shown.

starts to decrease. Around $2 \mathrm{kHz}$ the time between pulses is to short to get a complete bubble collapse and at the start of the heating pulse a small bubble can still be seen on the centre part of the heater. This results in a bubble continuously situated on the heater. At these frequencies the minimum energy required to create this permanent bubble is also shown in figure 4.35 .

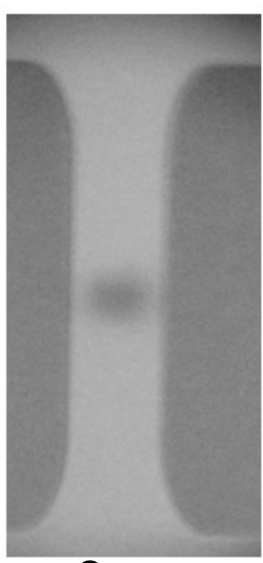

OHS

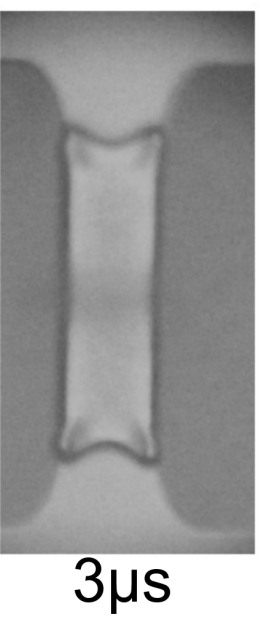

Figure 4.36: Images taken at $0 \mu \mathrm{s}$ and $3 \mu \mathrm{s}$ after the start of a $3 \mu$ s heating pulse for a $2 \mathrm{kHz}$ repetition frequency.

If a permanent bubble forms the necessary energy for nucleation and vapour sheet formation can still be determined. Figure 4.36 shows images taken at the start of the heating pulse $(0 \mu \mathrm{s})$ and at the end of the heating pulse $(3 \mu \mathrm{s})$. In the left image a small bubble can vaguely be seen on the central part of the heater and the right image shows a 
vapour sheet covering a large part of the heater. The bubble at the start of the heating pulse is much smaller than the heater and by supplying a certain amount of energy either nucleation of small bubbles over the entire heater or a vapour sheet formation can still be established and distinguished.

At frequencies above $2,5 \mathrm{kHz}$ the permanent bubble has grown to such size that the moment of nucleation or vapour sheet formation can not be determined any more. The bubble only grows during a heating pulse. This bubble growth is less reproducible. It can differ in time, location and speed. In this regime a large permanent bubble or a train of bubbles detaching from the heater surface show up if a continuous light source is used instead of the stroboscopic light source. The energy needed to create these bubbles decreases quickly with frequency to around $6 \mu \mathrm{J}$ at $10 \mathrm{kHz}$.

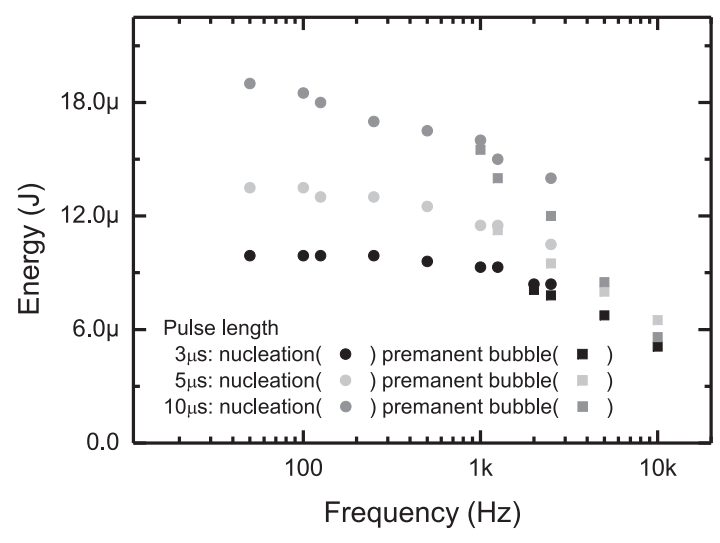

(a) Minimum energy

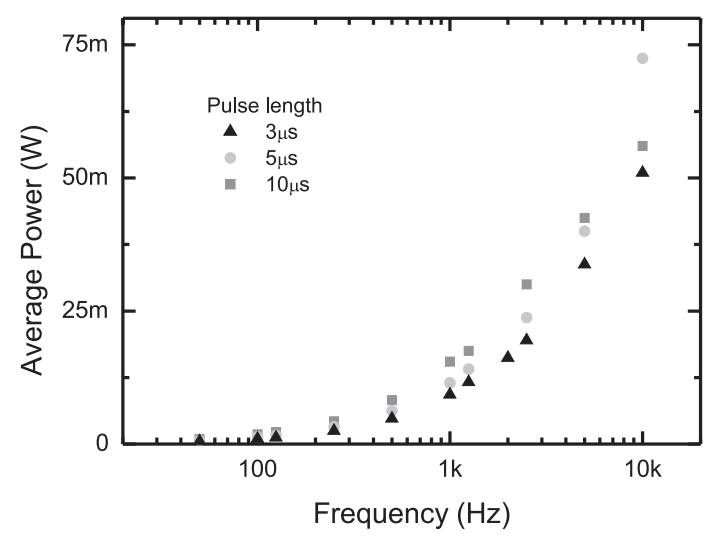

(b) Average power

Figure 4.37: Minimum energy to generate bubbles and the average power introduced into the system as a function of repetition frequency for 3 different pulse lengths.

In section 4.4.3 it was shown that the minimum energy to create a bubble decreases with pulse length. 4.37a shows the minimum energy as function of frequency for pulse lengths of $3 \mu \mathrm{s}, 5 \mu \mathrm{s}$ and $10 \mu \mathrm{s}$. At low frequencies the energy needed to get nucleation increases with pulse length. This difference decreases as the frequency decreases. At a $50 \mathrm{~Hz}$ frequency $10 \mu \mathrm{J}$ is needed to get nucleation at the end of a $3 \mu$ s pulse, but $19 \mu \mathrm{J}$ is needed for the $10 \mu \mathrm{s}$ pulse. At $10 \mathrm{kHz}$ about $6 \mu \mathrm{J}$ is required to generate bubbles for all three pulse lengths. The generation of a permanent bubble starts occur at $1 \mathrm{kHz}$ at $16 \mu \mathrm{J}$ per pulse for a $10 \mu \mathrm{s}$ pulse length and at $2 \mathrm{kHz}$ at a $8 \mu \mathrm{J}$ per pulse for a $3 \mu$ s pulse length. The average power is thus $16 \mathrm{~mW}$ in both cases. Figure $4.37 \mathrm{~b}$ shows that the average energy introduced into the system quickly increases with frequency. Although the energy per pulse thus decreases on average much more energy is introduced into the system at higher frequencies.

At low frequencies the temperature difference between the start of the heating pulse and nucleation temperature is relatively large and the pulse length has a large influence on the minimum energy required to get bubble nucleation. In section 4.4 .3 it was already shown that a shorter pulse requires less energy to generate a bubble and an identical behaviour is found here. As the frequency increases the temperature difference between 
the temperature at the start of the heating pulse and the nucleation temperature decreases due to an increase in average power input. As the frequency increases the minimum energy required for bubble generation will thus be lower and also the difference in minimum energy between the different pulse lengths will decrease. The increase in average power will not only result in an increase in the heater temperature at the start of the heating pulse, but the liquid surrounding the heater will increase in temperature during the heating as well as the cooling period. At some point the temperature will be high enough to sustain a gas bubble and the permanent bubble is formed. The generation of the gas bubble is directly related to the average power, the average power was equal for the $3 \mu \mathrm{s}$ and $10 \mu \mathrm{s}$ pulses.

\subsubsection{Effect on the bubble growth and collapse}

The moment the bubble collapse time exceeds the time in between the heating pulses the bubble nucleation and bubble dynamics can change considerably. In section 4.6.4 it was already mentioned that the bubble collapse time can be influenced by changing the pressure inside the cavity. An increase in cavity pressure will decrease the bubble collapse time. Figure 4.38 shows a bubble nucleation and collapse sequence at two different cavity
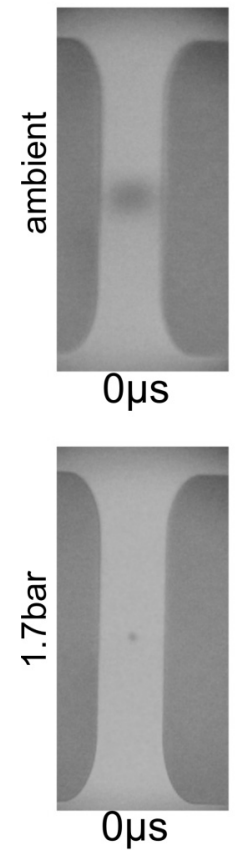
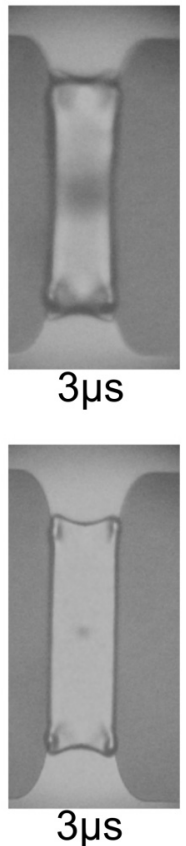
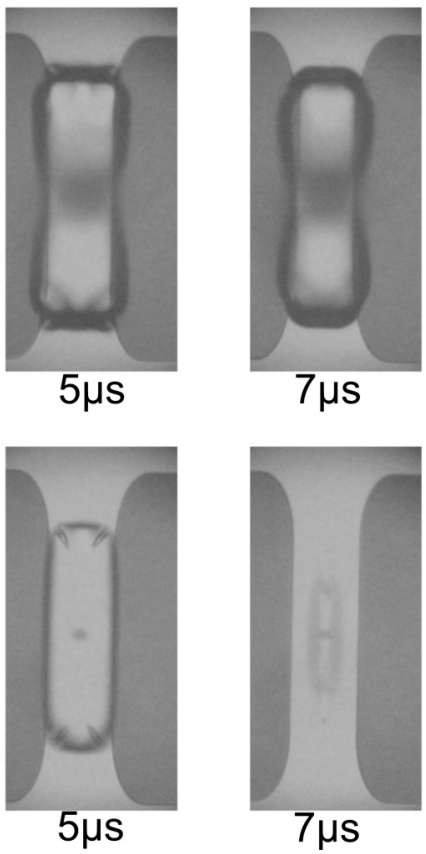

Figure 4.38: Sequence of bubble nucleation and collapse for a repetition frequency of $1 \mathrm{kHz}$ at ambient pressure and a at a pressure difference of 1.7 bar between the cavity and the outside pressure.

pressures at a repetition frequency of $1 \mathrm{kHz}$. The top row is taken at ambient pressure inside the cavity. Already at the start of the heating pulse $(0 \mu \mathrm{s})$ a gas bubble is situated on the heater. This shows that the bubble did not fully collapse after the last pulse. At the end of the heating pulse, around $3 \mu$ s after the start, the heater is covered by a vapour sheet. The gas bubble in the middle of the heater,which remained after previous pulses, 
can still be seen. The area covered by the vapour sheet and the geometry of the sheet look similar to the ones in the image taken at $3 \mu \mathrm{s}$ in figure 4.28. In the image taken at $5 \mu$ s it can be seen that the vapour sheet has slightly grown and became more rounded. In the centre of the heater the gas bubble from the previous pulses can still be distinguished and this bubble also covers a larger area. Furthermore the effect of this gas bubble on the vapour sheet clearly shows. The width of the vapour sheet is less in the centre and this does not show in the images taken at $5 \mu$ s in figure 4.28. The last image of the top row in figure 4.38 shows a further rounded vapour sheet. The gas bubble in the centre also seems to have grown even further in size. The bottom row of figure 4.38 is taken at 1.7bar pressure difference between the cavity and the outside pressure. The gas bubble, which remained from previous pulses, is much smaller than at ambient pressure. Its size stays the same during the first $7 \mu$ s of the pulse and it has no influence on the form of the vapour sheet during this time. The formation and collapse of the vapour sheet is similar to the behaviour as seen for the $1 \mathrm{~Hz}$ repetition frequency discussed in section 4.6.4.

At $1 \mathrm{kHz}$ repetition frequency and ambient pressure a small gas bubble permanently covers the heater surface. This gas bubble does not influence the bubble nucleation and vapour sheet formation. It does have some influence on the bubble dynamics. The appearance of the vapour sheet is a little bit different in the centre for images taken at $1 \mathrm{kHz}$ and $1 \mathrm{~Hz}$. Probably this will not have a large influence on the performance of the complete system. The fact that the gas bubble does not influence nucleation suggests that the bubble is not situated on the heater, but that it is floating at some distance above the heater. A gas bubble on the heater surface would enhance the nucleation at that location and the formation of a bubble would start at this location. The formation of a gas bubble at increased repetition frequencies is described by Varlomov [72]. Side view images shown in this paper confirm that the gas bubble is situated at some distance above the heater.

Furthermore a gas bubble will be situated in the part of the liquid with the highest temperature. During the heating pulse this will in general be the heater surface, but after the pulse, the heater will cool down faster than the surrounding liquid. The highest temperature can now be found in the liquid a small distance above the centre of the heater. At this location the gas will accumulate and the gas bubble will grow from this point.

A further increase in average power will increase the temperature of the heater and the liquid above the heater. A permanent bubble will be situated above the heater before, during and after the heating pulses. The size of this permanent bubble will depend on the average energy input into the system and on the pressure inside the cavity. An increase in cavity pressure will reduce the size of the gas bubble at the start of the heating pulse and will enhance the diffusion of gas in the liquid. The average power and the frequency at which the permanent bubble will start to influence the bubble dynamics thus increase with pressure. 


\subsection{Summary and conclusions}

The bubble generation and growth can be divided in several phases. In the first phase bubbles are nucleated. The bubble nucleation is imaged by stroboscopic imaging and during the heating pulse the heater temperature can be monitored by resistance thermometry. For bubble generation in ethanol two different nucleation regimes can be found. The single bubble regime and the spontaneous nucleation regime. For the single bubble regime the time and location of nucleation can be slightly different per pulse. Furthermore cracks and crevices in the heater surface or small gas bubbles present in the liquid or on the heater can act as nucleation sites. The spontaneous nucleation regime is characterized by a large number of small bubbles nucleating almost at the same time over a large part of the heater. The small bubbles almost instantly coalesce and form a vapour sheet. Although the nucleation is a random process, spontaneous nucleation is highly reproducible in time as well as location. The time of nucleation can be determined by stroboscopic imaging as well as the temperature versus time curve.

The time of nucleation decreases with the applied power. The energy per pulse on the other hand increases with the nucleation time. At high heat-fluxes nucleation early nucleation can occur due to an elevated temperature at regions with a high current density. Simulations of the current density can be used to predict the location of these sites. These same simulations can employed as a design tools to improve the heater layout and prevent the occurrence of early nucleation.

After nucleation the bubble will grow quickly with growth speeds of several tens of $\mathrm{m} / \mathrm{s}$. The maximum size and the bubble geometry depend on the heating parameters. The growth time, which is the time between the time of nucleation and the maximum size, decreases with with the applied heating power. The exact growth speed is hard to determine due to the structure of the bubble actuator. It is nearly impossible to get information on the height of the vapour sheet and therefore the volume of the bubble.

In the intermediate stage, bubble collapse and bubble growth alternate. The duration of this stage will depend on the heating power and the pulse length. If heating is continued the time between growth and collapses periods will increase and the difference in bubble size decreases. In most cases however the heating is stopped much earlier. It will depend on the amount of energy introduced into the system how long it will take before the bubble enters the last and final stage. In the sub-cooled liquid the vapour bubble will quickly condensate and disappear within a few microseconds. The images however show that bubble collapse takes much longer than one would expect from condensation. The bubbles probably contain other gasses. These gasses have to dissolve into the liquid and this will take much longer than vapour condensation.

The images discussed in this chapter correspond with images shown in a number of papers, where top as well as side view images of the bubbles where taken [72, 70, 71]. The images in these papers confirm the bubble geometries described earlier. The height of the initial vapour sheet is probably only several micrometers for the short heating pulses. After this a more rounded bubble grows from the vapour sheet. For the longer heating pulse a spherical bubble develops.

The pressure inside the cavity can be changed and this has a large effect on the duration of the last stage. The length of the final stage decreases with the applied pressure. The 
bubble collapse occurs faster and the residual bubbles take less time to disappear.

The micro-bubble actuator is designed to work at repetition frequencies of several $\mathrm{kHz}$. As the frequency increases the time between pulses and thus the time to cool down decreases. This results in an increase of the average temperature of the whole system. At low average power no bubble nucleation takes place and the increase in temperature is linear. At higher power bubble nucleation takes place and these bubbles lead to an increase in the heat transfer. The energy needed to nucleate a bubble decreases with frequency. The repetition frequency is limited by the formation of permanent gas bubbles in the liquid. These permanent bubbles are already present at the start of the heating pulse and are situated on the centre part of the heater. They are probably located a few micrometer above the heater in the volume of liquid with the highest temperature during the cooling period. The size and collapse time of these residual bubbles depend on the cavity pressure. An increase in cavity pressure increases the solubility of the gas and will result in a decrease in bubble size and bubble collapse time. The frequency at which these permanent bubbles start to grow will therefore decrease with cavity pressure. The generation and growth of these residual bubbles will also depend on the amount of dissolved gas in the ethanol. Intensive degassing of the ethanol before use will reduce the amount of residual gas and improve the performance of the actuator. 


\section{Chapter 5}

\section{Response of the membrane}

The response of the membrane to the generation of a bubble is treated in this chapter. The membrane speed and dynamic deflection are measured with a laser-vibrometer. The maximum deflection and the maximum repetition frequency are two important factors, which determine the overall performance of the actuator. A large number of parameters will effect these factors. The geometry and size of the membrane and the heaters, the pressure inside the cavity and the heating conditions are a examples of these parameters. Their influence on the membrane response will be investigated in this chapter. 


\subsection{Introduction}

In the previous chapter the dynamics of bubbles generated by short heating pulse was discussed. The bubbles are formed in a closed cavity with a flexible membrane on one side. When the bubbles are formed the pressure impulse generated inside the cavity will result in the deflection of the membrane.

The displacement of the membrane can be divided in two regimes; the static regime, where a constant deflection is caused by stress in the membrane or a pressure difference across the membrane, and the dynamic regime, where the membrane vibrates due to a time-dependent excitation. For the bubble actuator the excitation will be the pressure impulse generated by the nucleation and growth of bubbles. The deflection of the membrane can be measured by a laser Doppler vibrometer. After this initial deflection the bubble quickly collapses and the membrane will start to vibrate. The system is left in a damped free-vibration. The membrane displacement will be a superposition of a large number of membrane mode-shapes oscillating at their natural frequency. The amplitude of the vibrations will decrease in time due to damping.

In the first part of the chapter the static deflection, which changes with the pressure inside the cavity, is measured by a white light interferometer. From these measurement the young's modulus and initial stress of the membrane were determined. The dynamic displacement will be superimposed on the static deflection. The effect of parameters, such as membrane size, membrane geometry and cavity pressure, on the vibrations are discussed in the second part of the chapter. The initial deflection will determine the maximum amplitude of the displacement and influence the distribution of the modeshapes in the vibrations. The influence of the heating power, heater size, membrane size and cavity pressure on this initial deflection are investigated in the third part. In the last part of the chapter the effect of the repetition frequency on the performance of the actuator is discussed.

\subsection{Static deflection}

The static deflection is measured by white light interferometry. The Polytec MSA-400 has two modes for white light measurements; the shot-coherent and phase shift mode. The phase shift mode is relatively fast and can measure large areas, because it uses only the phase information of the interferometer signal. This mode was used to conduct the measurements on the static deflection. The samples are connected and filled conform the procedure discussed in section 3.6. After the syringe pump is stopped a delay of several minutes is maintained before the outlet valve is closed. This relaxation time ensures that the pressure inside the cavity, which is monitored indirectly by the pressure sensor between the inlet of the sample and the syringe pump, will be about the same as the outside pressure. There will be no flow through the tubing or in the cavity. The pressure inside the cavity and in the pressure sensor will thus nearly be the same. The pressure difference measured by the pressure sensor will closely correspond to the pressure difference across the membrane.

Figure 5.1 shows the surface scan for a circular membrane of $500 \mu \mathrm{m}$ diameter at a 
pressure difference of 1 bar. The maximum deflection is measured at the centre of the

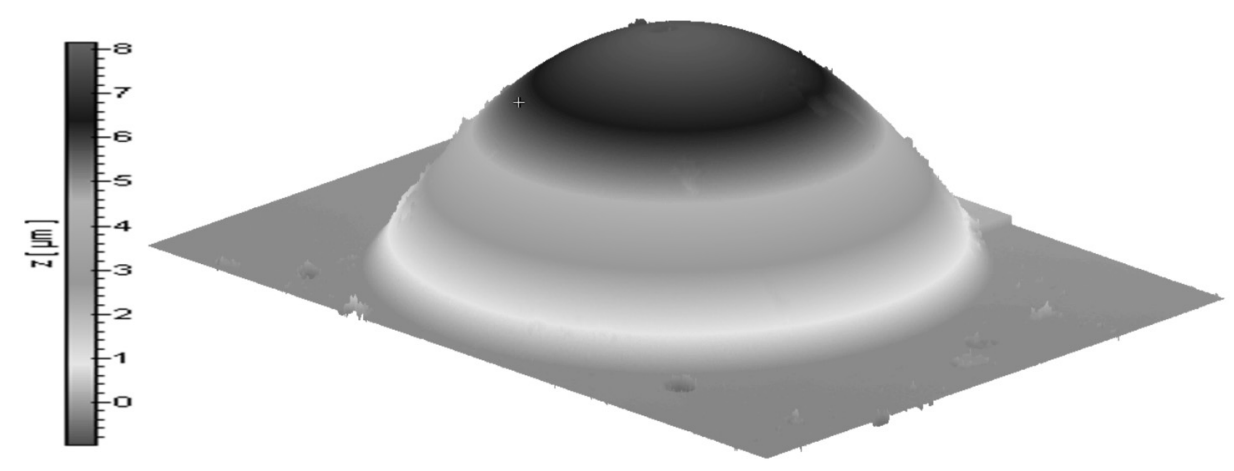

Figure 5.1: Deflection of a $500 \mu \mathrm{m}$ diameter membrane measured by a surface scan with a white light interferometer in phase shift mode.

membrane and is about $8 \mu \mathrm{m}$. Note that the membrane deflection is much smaller than the size of the membrane, but several times larger than the thickness of the membrane. This fact will become important when these results are compared to the values expected from theory. The measurement is repeated for pressure differences between 0 bar and 3 bar. Figure 5.2 shows the pressure difference as a function of maximum deflection.

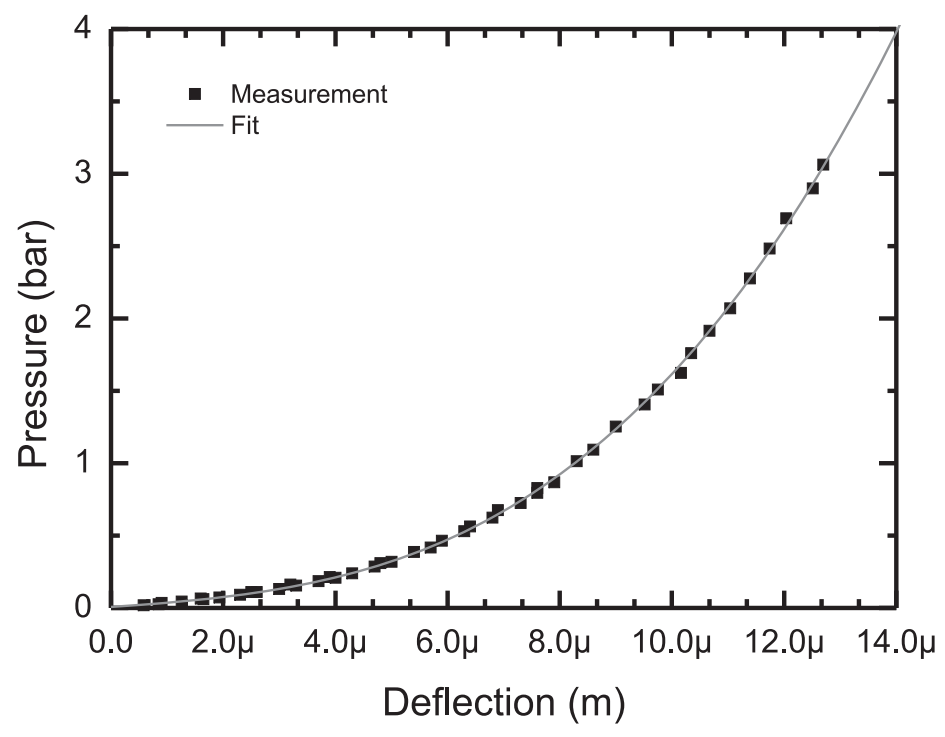

Figure 5.2: The static pressure difference across a circular 500 $\mu$ m diameter membrane as a function of the deflection at the centre of the membrane.

The measurements are compared to the theory discussed in chapter 2.5.1. Equation 2.33 was used for the fit shown in figure 5.2. The values found for the fitting parameters 


\begin{tabular}{|c|c|c|}
\hline parameter & value & error \\
\hline$c$ & $3.3 \times 10^{9}$ & $8.0 \times 10^{7}$ \\
$d$ & $1.3 \times 10^{20}$ & $7.6 \times 10^{17}$ \\
\hline
\end{tabular}

Table 5.1: Calculated fitting parameters.

are shown in table 5.1. These fitting parameters will later on be used to calculate the Young's modulus and initial stress in the membrane.

\subsubsection{Different membrane sizes}

Several membranes of different sizes and shapes were investigated under the white light microscope. Three square membranes with sizes of $1 \mathrm{~mm}, 500 \mu \mathrm{m}$ and $250 \mu \mathrm{m}$ and six circular membranes with diameters between $500 \mu \mathrm{m}$ and $200 \mu \mathrm{m}$ were examined. The results are shown in figure 5.3.

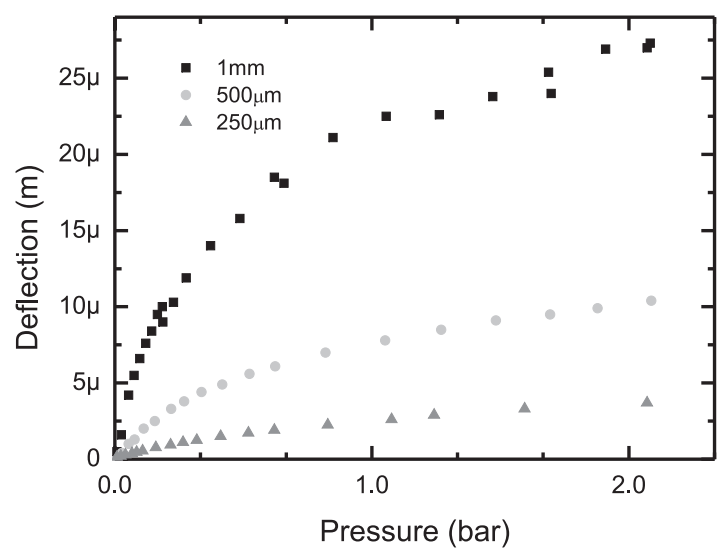

(a) Square membranes

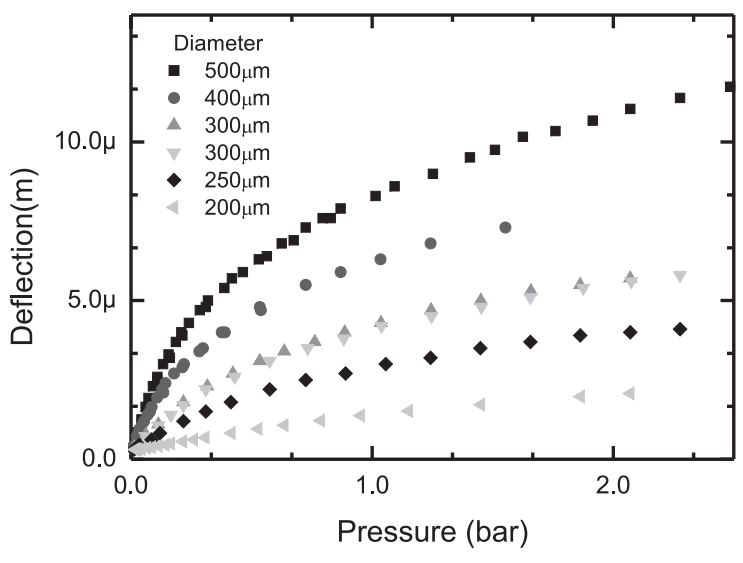

(b) Circular membranes

Figure 5.3: The measured deflection at the centre of the membrane for several square and circular membranes as a function of the pressure difference across the membrane.

In absence of a pressure difference none of the membranes show a considerable deflection at the centre and also the rest of the surface profile is smooth. All the membranes show an identical behaviour expected from theory; the deflection decreases with pressure difference and membrane size. The largest deflection of $27 \mu \mathrm{m}$ was measured for the $1 \mathrm{~mm}$ square membrane at 2 bar pressure difference. The slope of the deflection-pressure curve is smaller for the smaller membranes. For relatively small diameters at low pressure differences the deflection is in the same range as the membrane thickness and for these deflections a linear relation is expected from the theory.

Figure 5.4 shows the deflection versus pressure for a square membrane of $500 \mathrm{x} 500 \mu \mathrm{m}^{2}$ and a circular membrane with a $500 \mu \mathrm{m}$ diameter. The difference in deflection is relatively small, but increases with applied pressure. At 2 bar of static pressure difference the difference in deflection is $1 \mu \mathrm{m}$, which is about $10 \%$ of the total deflection. A difference 


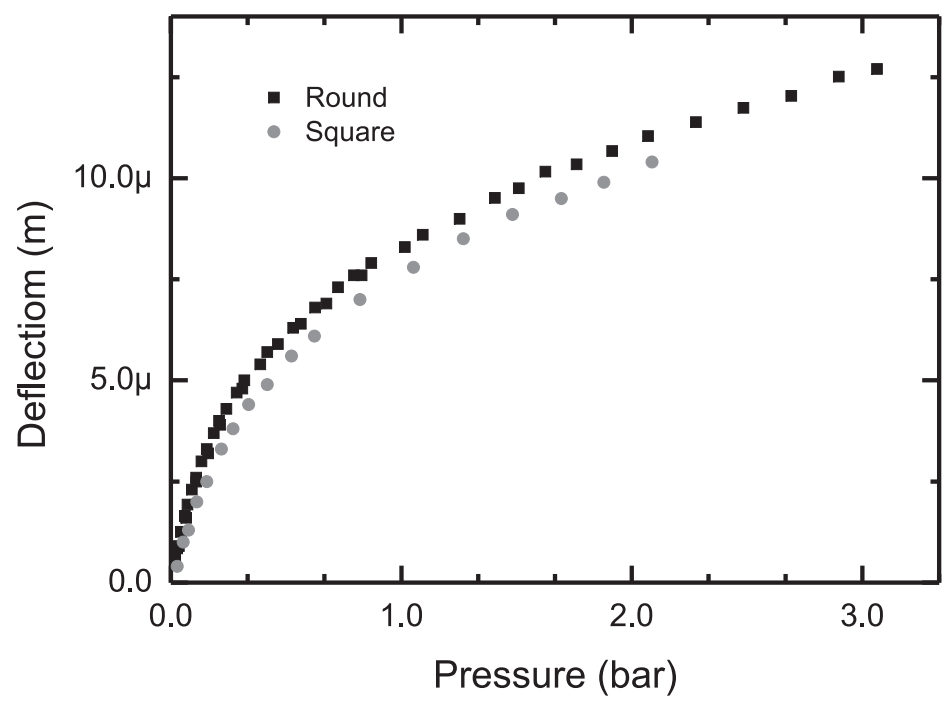

Figure 5.4: The deflection for a square and a circular membrane of $500 \mu \mathrm{m}$.

is expected from the theory described in [83], but it predicts a smaller deflection of the circular membrane in comparison to the square membrane. In this case, however, the difference is probably more related to the fact that the membrane sizes specified in the design are used. The actual membrane size, especially the square membrane size, can deviate several micrometers from the defined size.

In spite of these differences figures 5.3 and 5.4 prove that we can use the general theory to describe the deflection of the round, as well as square, membranes under static pressure. In the previous paragraph the measured data is compared to the theory by a

\begin{tabular}{|c|c|c|c|c|}
\hline diameter & $c$ & $d$ & $\sigma_{0}$ & $E$ \\
\hline $2.0 \times 10^{-4}$ & $6.2 \times 10^{9}$ & $1.0 \times 10^{22}$ & $-3.4 \times 10^{7}$ & $3.5 \times 10^{11}$ \\
$2.5 \times 10^{-4}$ & $1.6 \times 10^{10}$ & $2.2 \times 10^{21}$ & $4.6 \times 10^{7}$ & $1.8 \times 10^{11}$ \\
$3.0 \times 10^{-4}$ & $9.7 \times 10^{9}$ & $8.0 \times 10^{20}$ & $4.6 \times 10^{7}$ & $1.4 \times 10^{11}$ \\
$3.0 \times 10^{-4}$ & $1.1 \times 10^{10}$ & $8.4 \times 10^{20}$ & $5.3 \times 10^{7}$ & $1.4 \times 10^{11}$ \\
$4.0 \times 10^{-4}$ & $4.5 \times 10^{9}$ & $3.0 \times 10^{20}$ & $4.0 \times 10^{7}$ & $1.6 \times 10^{11}$ \\
$5.0 \times 10^{-4}$ & $3.3 \times 10^{9}$ & $1.3 \times 10^{20}$ & $4.8 \times 10^{7}$ & $1.7 \times 10^{11}$ \\
\hline
\end{tabular}

Table 5.2: Calculated fitting parameters and young's modulus for the circular membranes.

fit to equation (2.33). Table 5.2 shows the values for the fitting parameters of the square membranes. Both the fitting parameters increase with membrane size. Equation(2.34) is used to calculate the Young's modulus for the membranes from the coefficients $d$. This Young's modulus was utilized to calculate the initial stress by using equation(2.34). The young's modulus and the initial stress should be nearly identical and independent of the membrane size, because all the samples came from the same wafer and went through the same process steps. Almost all values found for the Young's modulus and the initial stress show good correspondence with each other. Only for the smallest $200 \mu \mathrm{m}$ diameter 
membrane the value for the Young's modulus is much larger and the initial stress has a negative value. These deviations occur, because the measured deflections have values close to the thickness of the membrane and most of the data points are in the linear deflection regime. The error in $d$ is large due to the limited number of measurements in the cubic regime. Therefore the young's modulus can't be determined with high accuracy and the error in the calculated initial stress will also be large.

The average Young's modulus is $1.58 \times 10^{11} \mathrm{~Pa}$ with an standard error of $8.0 \times 10^{9} \mathrm{~Pa}$ and the average initial stress is $4.63 \times 10^{7} \mathrm{~Pa}$ with a standard error of $2.17 \times 10^{6} \mathrm{~Pa}$. In these calculations the data for the $200 \mu \mathrm{m}$ diameter membrane is omitted. Although they can change considerably with the method of deposition and during different process steps, the values found here are comparable to values of 100 to $300 \mathrm{GPa}$ for the young's modulus and -50 to $170 \mathrm{MPa}$ for the initial stress found in literature [105, 106, 107].

\begin{tabular}{|c|c|c|c|}
\hline size & $c$ & $d$ & $E$ \\
\hline $1.0 \times 10^{-3}$ & $3.3 \times 10^{8}$ & $9.1 \times 10^{18}$ & $1.9 \times 10^{11}$ \\
$7.5 \times 10^{-4}$ & $4.7 \times 10^{9}$ & $1.4 \times 10^{20}$ & $1.9 \times 10^{11}$ \\
$5.0 \times 10^{-4}$ & $3.1 \times 10^{10}$ & $1.7 \times 10^{21}$ & $1.4 \times 10^{11}$ \\
\hline
\end{tabular}

Table 5.3: Calculated fitting parameters and young's modulus for the square membranes.

The data for the square membranes is also fitted to equation (2.33) and the values found for $c$ and $d$ are shown in table 5.3. Because no analytical solutions exist and the approximations found from literature differ from each other, equation (2.34) was also used to calculate the Young's modulus for the square membranes. The values found for the square membranes are comparable to values found for the circular membranes. For a square membrane the initial stress will change the relation between pressure and deflection, but this influence is even harder to determine and thus no attempts were made to calculate the initial stress for the square membranes.

\subsection{Dynamic deflection}

The dynamic deflection of the membrane is determined with a laser Doppler vibrometer. This is a two-beam interferometric device, which detects the frequency difference between an internal reference beam and a measurement beam. The measurement beam is focused on the the target and scattered back to the interferometer. The out of plane speed from the moving target is calculated from the frequency change. A Polytec MSA-400 Scanning Vibrometer was used to measure the speed and deflection at a certain point on the membrane in real-time. The vibrometer was triggered by the home-made pulse generator at the start of the heating pulse. This vibrometer also has the ability to make a surface scan and conduct identical measurements on a large numbers of points on the membrane. Combining the data from such a scan gives more information on the behaviour of the entire membrane during a single pulse.

Bubbles are produced by microsecond heating pulses and the pressure impulse generated by the nucleation and growth of the bubble will result in a dynamic displacement. Figure 5.5 shows the displacement of a circular membrane with a $500 \mu \mathrm{m}$ diameter due 


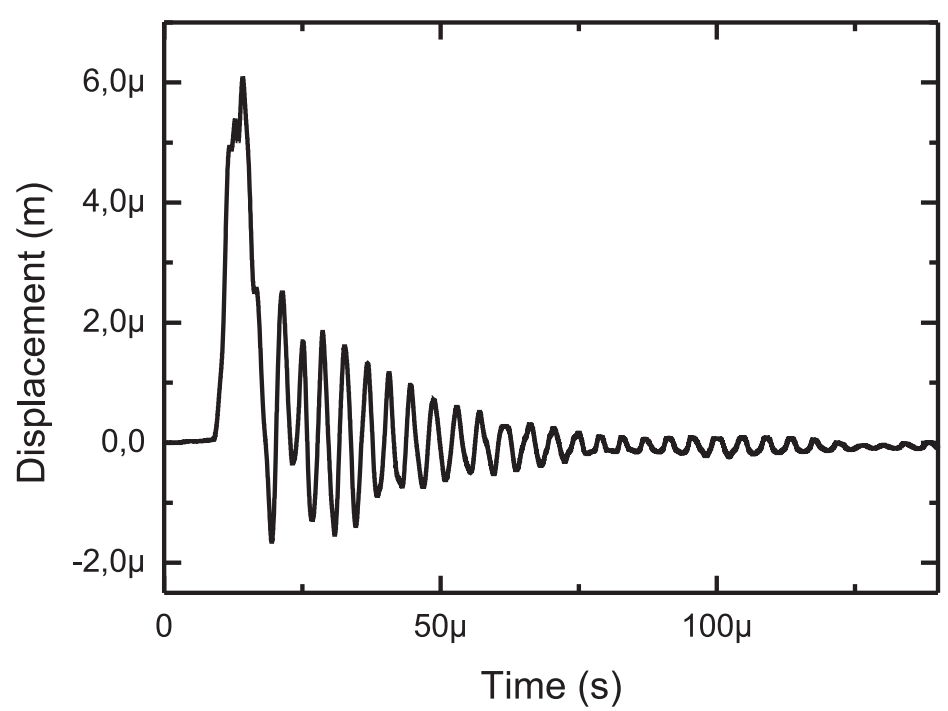

Figure 5.5: Displacement at the centre of a circular membrane during and after a $10 \mu \mathrm{s}$ heating pulse.

to a $10 \mu$ s pulse with a heating power of $1.7 \mathrm{~W}$. The sudden formation of a bubble in the liquid will result in a fast increase in deflection. The initial deflection is a superposition of a large number of mode-shapes. After this initial deflection the bubble will quickly collapse and the system will be in damped free-vibration. Each mode-shape will start to oscillate at is own natural frequency. Mode-shapes, which require a net-volume change, will dissipate energy faster due to expansion and compression of the liquid. However, for mode-shapes, where only a small volume is shifted and thus no net-volume change takes place, the damping is relatively low. The amount of damping for a mode-shape will thus determine the number of oscillations of a particular frequency. In figure 5.5 a initial deflection of $6 \mu \mathrm{m}$ was measured $14 \mu \mathrm{s}$ after the heating starts. After this initial deflection the oscillations quickly dampen and $120 \mu$ s later the vibrations have decreased to a maximum amplitude of less than $50 \mathrm{~nm}$.

The mode-shape at a natural frequency is resolved from the profile at that particular frequency. The profile is determined from the data of a surface scan. This method can only be used if the response of the membrane after a heating pulse is identical for each measurement. Figure 5.6 shows the profile of a membrane at the moment of maximum deflection. The displacement is measured for a large number of points spread across the membrane and the combination of the data gives a profile of the moving membrane in time. To determine the complete profile more than 50 individual measurements made by a surface scan were combined. The smoothness of the profile proves that explosive evaporation and the subsequent membrane deflection is highly reproducible. All points on the grid correspond with an individual measurement and such a smooth profile can only occur when all points reach their maximum displacement exactly at the same time. 


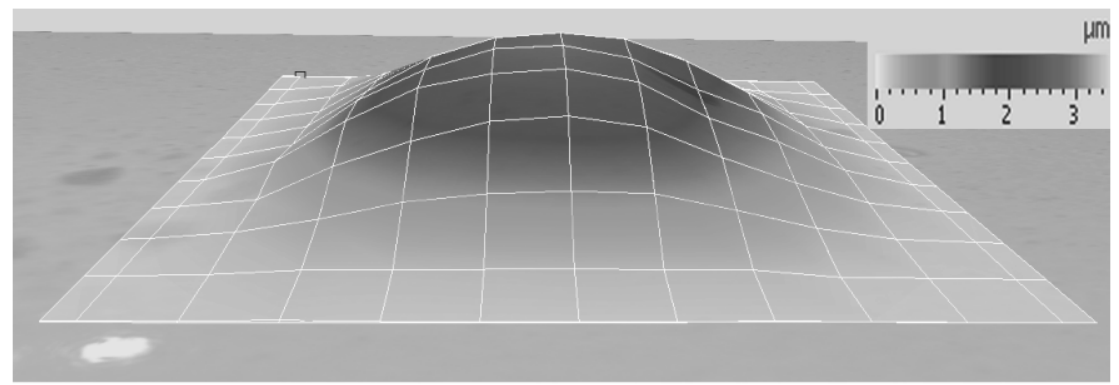

Figure 5.6: Surface scan of the $250 \mu \mathrm{m} \times 250 \mu \mathrm{m}$ membrane at the moment of maximum deflection.

\subsubsection{Effect of the membrane geometry}

The initial deflection and the response of the membrane after excitation by a bubble will depend on the size, geometry and properties of the membrane. Samples with a square and a circular geometry were investigated. The response of a membrane to a heating pulse was determined by a surface scan with the laser vibrometer. The contribution of each frequency in the membrane response can be determined by calculating the fast Fourier transform (FFT) of the displacement signal. The FFT's from the different points of a surface scan can be added together and the average FFT is calculated by dividing the sum of all the individual FFT's by the number of points. This average FFT shows the distribution of the membrane displacement as a function frequency. By coupling the displacements at a certain frequency for the different measurement points, the profile of the membrane at that frequency can be displayed. This should correspond to a membrane mode-shape at a natural frequency.

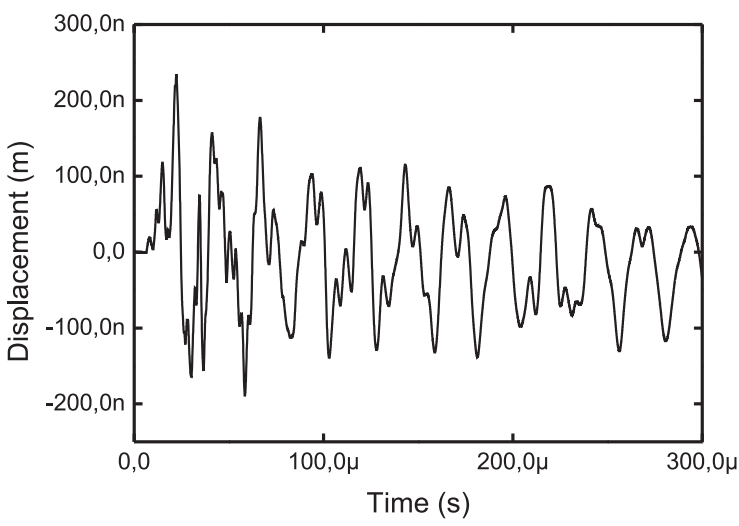

(a) Displacement

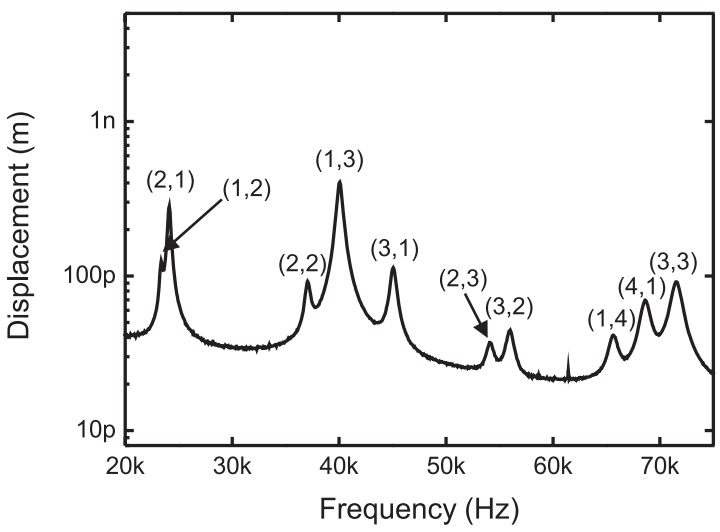

(b) Average Fourier transform

Figure 5.7: The displacement at the centre and the average Fourier transform of measurements across the entire $1,5 \times 1,5 \mathrm{~mm}^{2}$ square membrane for a $10 \mu$ s heating pulse. 


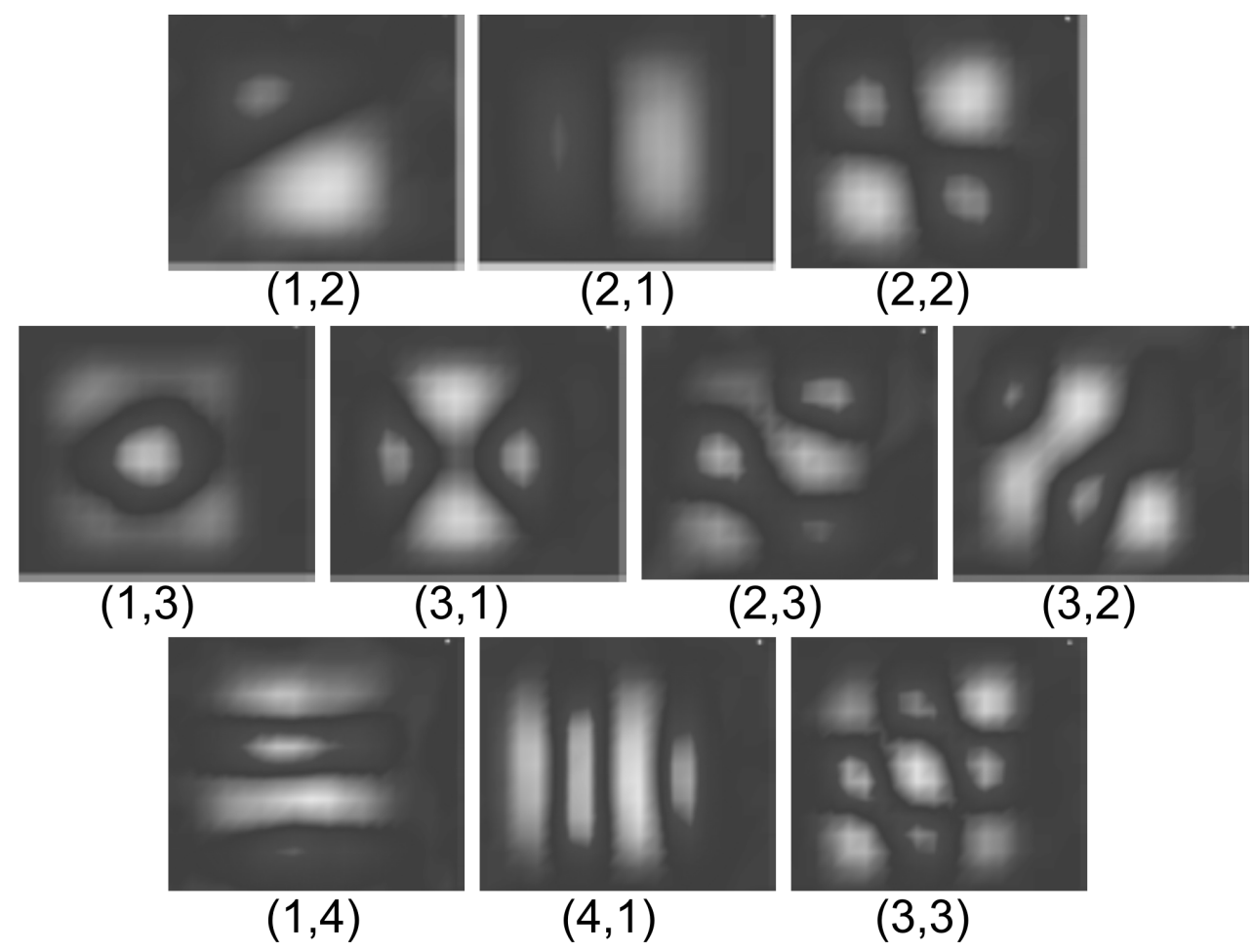

Figure 5.8: Images of the mode-shapes measured at the peaks of the frequency spectrum shown in figure $5.7 \mathrm{~b}$.

Figure 5.7 a shows the displacement in the centre of a square $1,5 \mathrm{x} 1,5 \mathrm{~mm}^{2}$ membrane. A maximum displacement of $230 \mathrm{~nm}$ at $22 \mu \mathrm{s}$ after the start of the heating pulse was detected. After this maximum the oscillations decrease in time. Figure 5.7b shows the average Fourier transform of the displacement taken from a surface scan of 50 points spread equally over the membrane. The frequency spectrum in figure $5.7 \mathrm{~b}$ shows several peaks. Each peak corresponds to particular natural frequency $(m, n)$ of the membrane. Figure 5.8 shows the membrane profiles at the frequencies corresponding to the different peaks. The profiles are compared to mode-shapes shown in figure 2.22. From this comparison it is determined to which mode the frequency corresponds. The first two profiles correspond to the $(1,2)$ and $(2,1)$ mode-shapes, where half of the membrane has an out of plane deflection in anti-phase with the other half of the membrane. The other mode-shapes are also similar to the ones expected for a square membrane. All modes, which require no net volume-change, are present. Mode-shapes, like the fundamental mode $(1,1)$, require a net volume change in the cavity; these are highly dampened and are not present in the frequency spectrum.

The distribution of the mode-shapes and the exact profile at a particular frequency will depend on the initial excitation. The initial deflection due to the pressure impulse of the bubble is largest in the centre part of the membrane. Mode-shapes with a relatively large centre deflection, like the $(1,3)$ mode-shape, will thus be particularly present in the total vibration.

A square membrane would have an identical natural frequency for the $(1,2)$ and $(2,1)$ modes. Figure 5.7b, however clearly shows a difference in frequency between the $(1,2)$ 
and the $(2,1)$ peaks and also for similar modes at higher frequencies (for example the $(1,4)$ and $(4,1)$ mode-shapes) a difference in frequency occurs. This difference arises, because the membrane is not square, but rectangular. The ratio between the sides determines the difference in frequency between the modes.

The theory predicted four extreme cases for the $(1,3)$ and $(3,1)$ mode-shapes of a square membrane. For a rectangular membrane the natural frequency for the $(1,3)$ and $(3,1)$ mode shapes will be different. Both mode-shapes can be related to one of the extreme cases. The $(1,3)$ mode-shape looks similar to the case, where $\mathrm{A}=\mathrm{B}$, and the $(3,1)$ mode-shape looks like the $\mathrm{A}=-\mathrm{B}$ case. A mode-shape, where either $\mathrm{A}=0$ or $\mathrm{B}=0$, requires a net-volume change and therefore will be highly dampened. These mode-shapes will thus not be found in the spectrum.

Not only square membranes were investigated, but also circular membranes. Figure 5.9 shows the displacement and the average Fourier transform of the displacement for a circular membrane with a $500 \mu \mathrm{m}$ diameter. Also for this membrane the peaks in

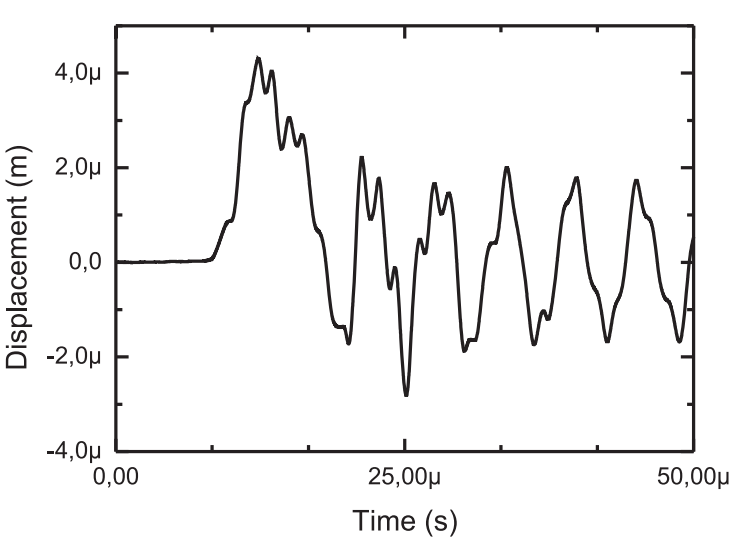

(a) Displacement

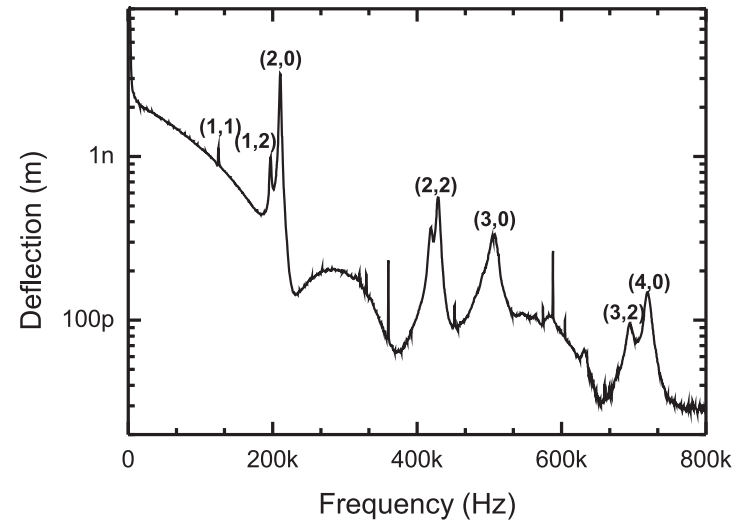

(b) Average Fourier transform

Figure 5.9: Displacement at the centre of the membrane and the average Fourier spectrum of the measurements across a circular membrane.

the frequency spectrum match to the different natural frequencies. Figure 5.10 shows the profiles at the different peaks in the frequency spectrum. The profiles show a good correspondence with the mode-shapes shown in 2.21 and from this the particular modeshape is determined. For a circular membrane only the modes with a circular symmetry show a centre deflection. These mode-shapes will be excited the most by the pressure impulse. The fundamental mode will be highly dampened and does not appear in the spectrum. The next mode with a circular symmetry is the $(2,0)$ mode-shape. This mode does not lead to a net volume change and has a relatively large centre deflection, therefore it has the largest amplitude in the spectrum. The other mode-shapes with a circular symmetry also don't lead to a volume change and can thus be found in the frequency spectrum.

The $(1,2)$ and $(2,2)$ and $(3,2)$ have two nodal lines through the centre and thus don't have a centre deflection. The generated bubbles and the initial deflection will not be completely circular, therefore the mode modes with two nodal lines are also excited, 


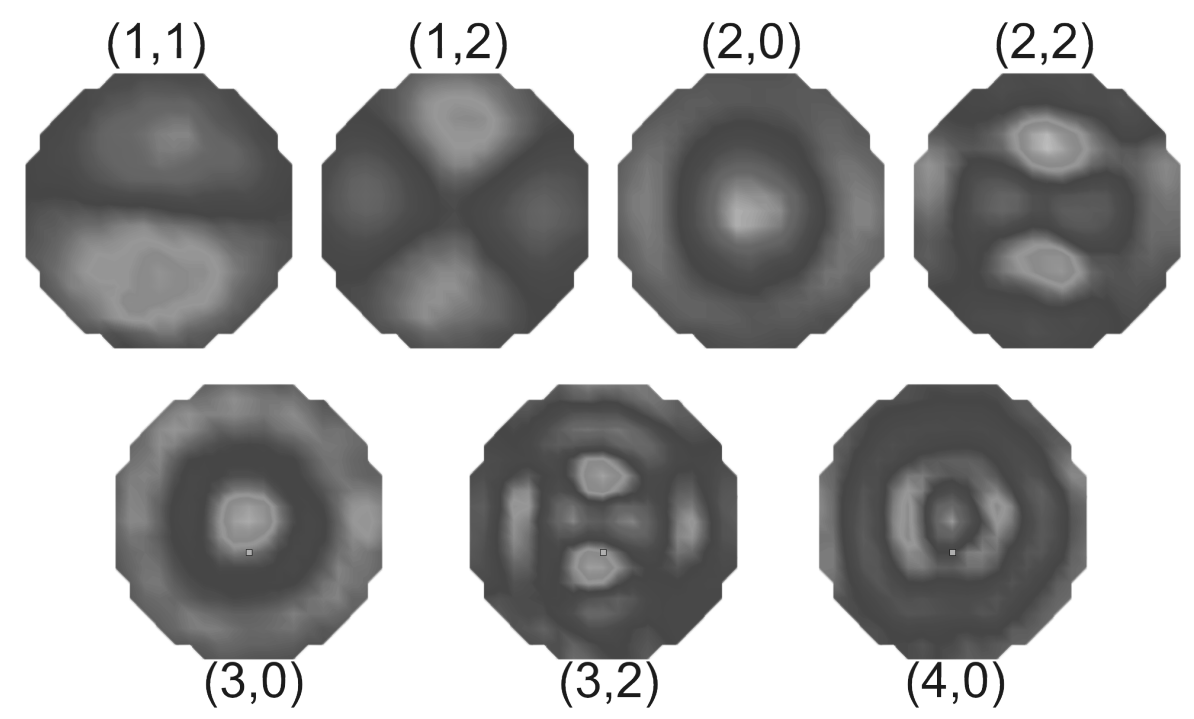

Figure 5.10: Images of the mode-shapes measured at the peaks in the frequency spectrum shown in figure $5.9 \mathrm{~b}$.

but to a lesser extent. The $(1,1),(2,1)$ and $(3,1)$ mode-shapes have a single nodal line though the centre of the membrane and are be hardly excited, only the $(1,1)$ mode could be distinguished. Excitation of these modes can occur, for example, if the maximum deflection does not occur exactly at the centre of the membrane.

\subsubsection{Effect of the membrane size}

The theory of chapter 2 predicts that natural frequencies should depend on membrane size. The frequency scales with the radius of the membrane following equation:

$$
f=a r^{b}
$$

The value of $b$ can have a value between -1.5 and -2.5 , if the membrane is considered to be a plate or an ideal membrane, respectively. Figure 5.11 shows the frequency of the $(1,0)$ and the $(2,0)$ mode as a function of the membrane radius. In general the frequencies of both modes decrease with membrane size. The dotted lines show a fit to equation 5.1. For the largest membrane with a radius of $250 \mu \mathrm{m}$ and for the $(2,0)$ mode-shape of the $100 \mu \mathrm{m}$ membrane the results deviate from the fit. The values found for the natural frequency are higher than expected. For relatively large membranes the fundamental frequency is low and the difference in frequency between the individual modes is small. Often the peaks overlap and the exact natural frequency is difficult to determine. For the smallest membranes the resolution of the measurements make it more difficult to determine the exact mode, especially the different higher modes are often difficult to distinguish. Therefore the uncertainty in the measured frequency for large membranes and the higher modes of the smallest membranes is much higher.

The value for the fitting parameters are shown in table 5.4. In the fitting procedure the values for the membrane with a $250 \mu \mathrm{m}$ radius were therefore omitted due to the large uncertainty. The values found for $b$ of -1.8 and -2.0 for the different mode shapes lies 


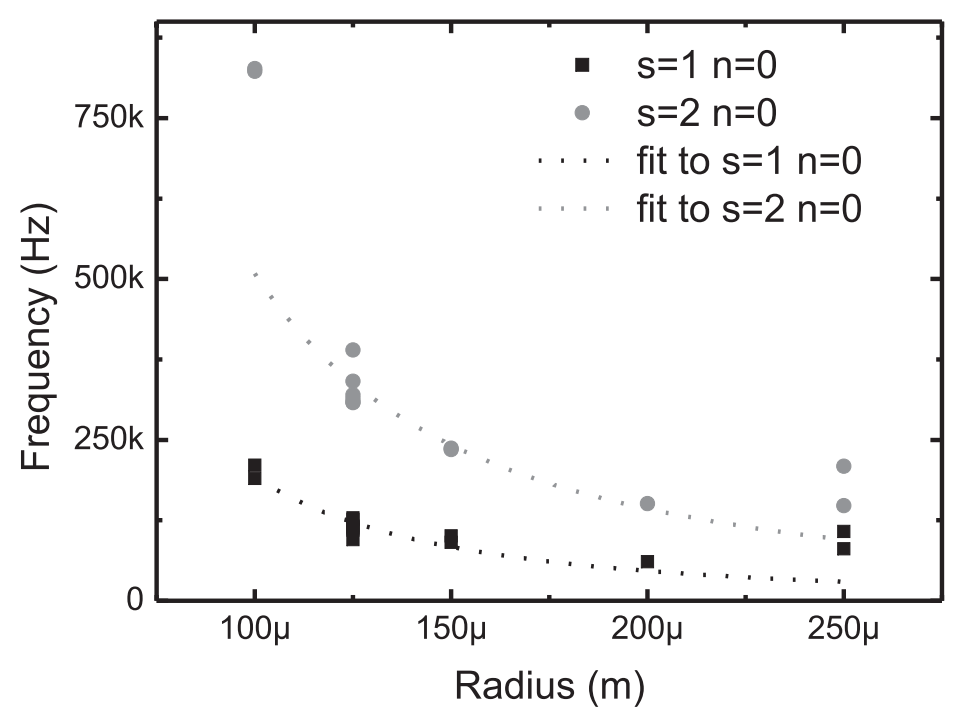

Figure 5.11: The natural frequency of the $(1,0)$ and the $(2,0)$ mode shape for different circular membranes with a radius between $100 \mu \mathrm{m}$ and $250 \mu \mathrm{m}$. The measured frequencies were fitted to equation (5.1).

\begin{tabular}{|c|c|c|c|c|}
\hline & $a$ & $b$ & error $a$ & error $b$ \\
\hline $\mathrm{s}=1, \mathrm{n}=0$ & $1 \times 10^{-3}$ & -2.0 & $2 \times 10^{-3}$ & 0.2 \\
$\mathrm{~s}=2, \mathrm{n}=0$ & $2 \times 10^{-2}$ & -1.8 & $8 \times 10^{-2}$ & 0.4 \\
\hline
\end{tabular}

Table 5.4: Values found for the fitting parameters of equation (5.1)

within the range between -1.5 and -2.5 , which was expected from theory. The value found for $a$ has a very large error. This large error can have several causes. The relation between membrane size and natural frequency is probably more complicated than assumed here and extra terms in equation 5.1 have to be added. The uncertainty in the measured frequencies, however, also contributes to the error and this makes an improvement of the model difficult. The exact thickness of the membrane and the initial stress will also have an influence, but all the measured devices came from the same wafer and went to identical fabrication steps. Therefore the difference in thickness and initial stress will only be a few percent. This will not have a large influence on the relation between membrane size and natural frequency. It however can be concluded that the natural frequency increases with decrease in membrane radius. On estimate the frequency of the $(1,0)$ and $(2,0)$ mode-shape scale with the inverse square of the radius.

\subsubsection{Effect of the applied heating power}

The previous chapter has shown that the time of nucleation and the size of the bubble change with the applied heating power. The initial deflection, which will be considered in great detail in section 5.4, is expected to change considerable with applied power and this will influence the response of the membrane. Figure 5.12 shows the average Fourier transform of the deflection for a $1,5 \times 1,5 \mathrm{~mm}^{2}$ membrane. The deflection was measured 


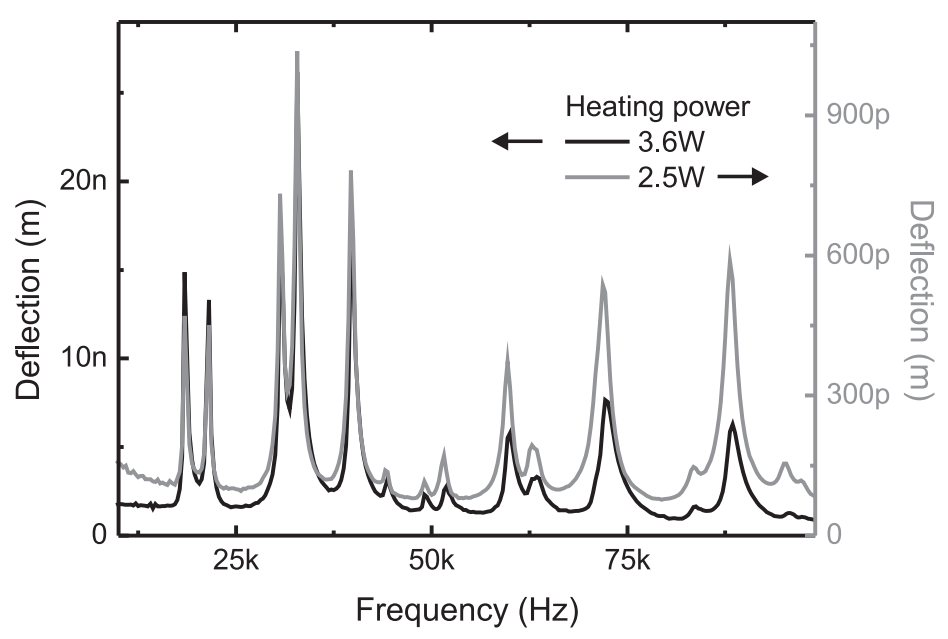

Figure 5.12: Average Fourier transform of the deflection for two different applied heating powers.

for two different heating powers. For the highest power of $3.6 \mathrm{~W}$ the first 5 peaks in the Fourier transform are nearly 30 times larger than for a power of $2.5 \mathrm{~W}$. For the rest of the peaks the difference is smaller, but the height of the peaks for a power of $3.6 \mathrm{~W}$ is still more than 10 times larger than for the $2.5 \mathrm{~W}$ heating power. The frequency corresponding to the different peaks is identical for both heating powers. In the frequency range measured here the natural frequencies of the system are thus independent of the heating power. As expected the frequency of the vibrations is not influenced by the bubble generation and growth, but will only depend on the membrane size, geometry and material properties. The amplitude of the vibrations, however, does depend on the applied power, it increases due to the increase in bubble volume at higher applied powers. The lower frequencies show a larger increase in amplitude than the high frequency mode-shapes. An increase in heating power thus also leads to a shift in the distribution of the mode-shapes. Low frequency mode-shapes are excited more.

\subsubsection{Effect of the cavity pressure}

The dynamic deflection of the membrane is influenced by the cavity pressure in two ways. Firstly an increase in pressure difference across the membrane leads to a static deflection and an increase in tension in the membrane. Secondly the bubble generation and bubble dynamics are influenced by the pressure. Figure 5.13 shows the average Fourier transform of the deflection for a $1,5 \times 1,5 \mathrm{~mm}^{2}$ membrane at three different cavity pressures under identical heating conditions. An increase in pressure difference leads to an increase in resonance frequency and a decrease in amplitude. For the fourth peak, which corresponds to the $(3,1)$ mode, the amplitude decreases from $1 \mathrm{~nm}$ to $0.4 \mathrm{~nm}$ and the resonance frequency increases from $32 \mathrm{kHz}$ to $81 \mathrm{Khz}$ for an increase in pressure from atmospheric pressure to difference of 0.8 bar. The relative decrease in amplitude is not identical for all the peaks. The peaks corresponding with mode shapes with a relatively large centre deflection become more prominent with increasing pressure. This change 


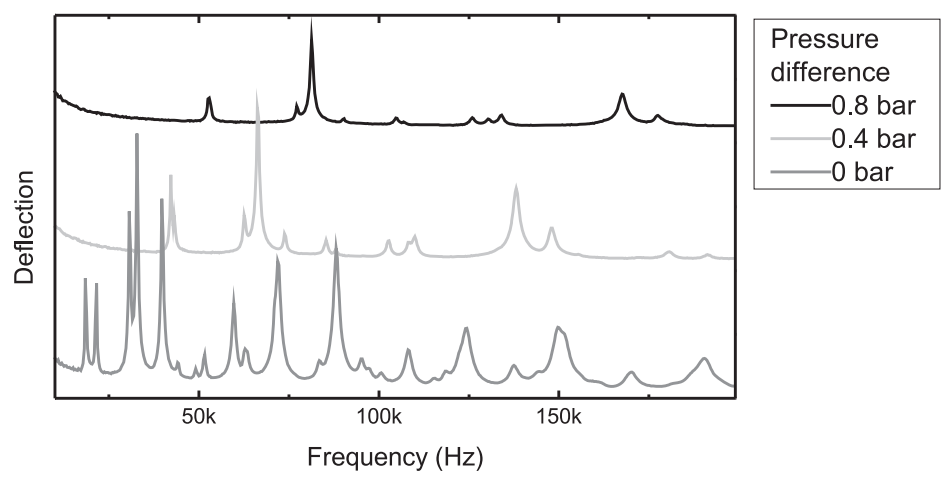

Figure 5.13: Average Fourier transform of the deflection at three different pressure differences across the membrane.

in distribution can be related to several causes. In the previous section it was already concluded that the bubble generation and bubble dynamics can lead to a shift in the distribution of the mode-shapes. The change in tension will also lead to change in this distribution. From these measurements could be concluded that the mode-shapes with large centre deflection are less affected by the increase in membrane tension. This is related to the fact that the bending energy is proportional to the second derivative of the deflection, while the energy associated with the axial tension is proportional to the first derivative. The second derivative becomes dominant in high modes.

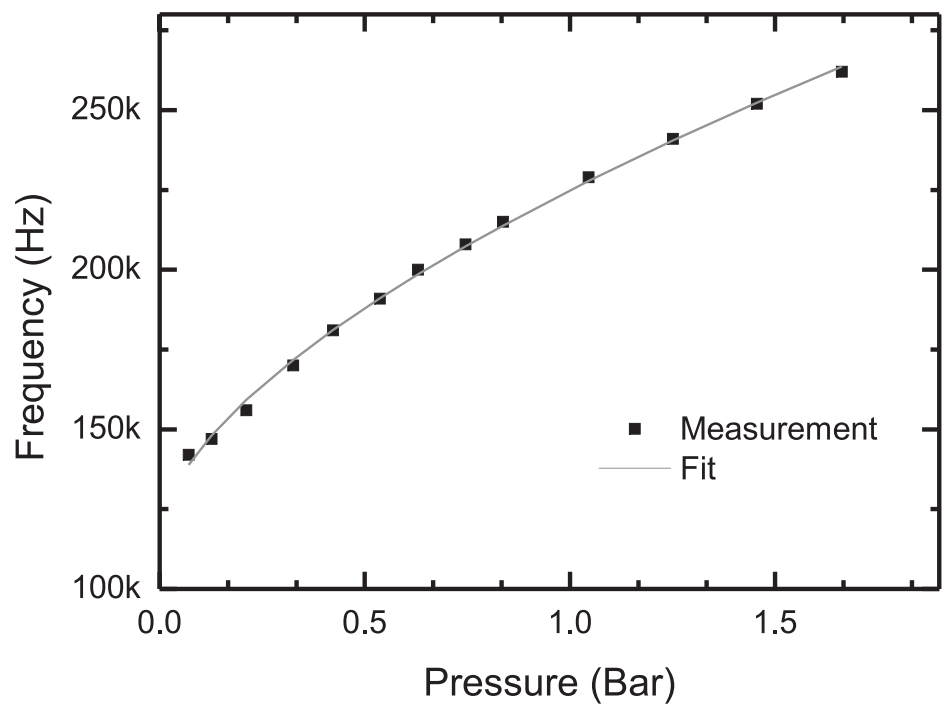

Figure 5.14: Natural frequency of a single mode-shape as a function of the pressure difference across the membrane. Equation (5.2) was used in the fitting procedure.

The effect of pressure on the natural frequency was investigated for a single frequency. In this case a circular membrane with a diameter of $400 \mu \mathrm{m}$ was used. Figure 5.14 shows the frequency of the $(2,0)$ mode shape as a function of applied cavity pressure. The frequency increases from $142 \mathrm{kHz}$ at atmospheric pressure to $262 \mathrm{kHz}$ at a pressure difference of 1.7 
bar across the membrane. According to the theory discussed in section 2.5.2 the resonance frequency scales with the square root of the membrane tension or the flexural rigidity for respectively a membrane or a plate. An increase in cavity pressure will not only result in a static deflection of the membrane, but will also change the stresses in the membrane. The bending stress will scale linearly with the deflection and the tensile stress will scale with the square of the deflection. The total stress in the membrane will have considerable effect on the natural frequency. Furthermore the stress is not equally distributed over the membrane and its effect on each mode can be different. Therefore no simple solution for the relation between resonance frequency and pressure exists. In order to do some predictions on the change of resonance frequency the following equation was used in the fit shown in 5.14

$$
f=C_{1}+C_{2} P^{n}
$$

where $f$ is the measured resonance frequency, $P$ is the measured pressure difference across the membrane, $C_{1}, C_{2}$ and $n$ are fitting constants. A value of $117 \mathrm{kHz}$ with an error of $4 \mathrm{kHz}$ was found for $C_{1}$. This frequency can be seen as the natural frequency at ambient pressure inside the cavity. The value of $n$ gives an idea about the relation between the pressure and the natural frequency. In this case a value of 0.6 with and error of 0.03 was found for $n$. A value of 1 would mean a linear relation and for a value of 0.5 the frequency scales to the square root of the pressure. The value of $C_{2}$ will depend on the radius of the membrane, the specific mode and the material properties of the membrane and the liquid. For $C_{2}$ a value of 107 with an error of 4 was found. Powell et al. [108] examined the effect of pressure on the natural frequency of a circular diaphragm. Curves shown in this paper all displayed a rise of frequency with pressure. The shape of the curves resembles a saturation curve, but the for higher harmonics the corresponding features occur at higher pressures. The curve shown in figure 5.14 is similar to middle part of the curves shown in the mentioned paper, where a relatively large change in frequency occurs with a change in pressure. Because of the limited pressure range and the fact that the relation between pressure and frequency was not measured for other modes or other membrane types, no further comparison can be made. For this particular mode shape and range of pressures the frequency scales to the power of 0.6 with the pressure. For other mode-shapes an increase in frequency can be expected with an increase in pressure, but further investigation have to be conducted to find the exact relation.

\subsection{Initial dynamic deflection}

The initial deflection has a large influence on the response of the membrane. It determines the amplitude and the distribution of the modes-shapes of the vibrations. In most cases the maximum displacement of the membrane and the top of the initial deflection peak are equal. The initial deflection is therefore also an important performance parameter of the actuator. The average speed of the deflection can be calculated by taking the difference between the onset of deflection and the time of maximum displacement. The influence of the heating parameters, such as heating power, pulse length and heater area as well as the membrane size and cavity pressure on the size of the initial deflection are investigated in this section. 


\subsubsection{Nucleation and the maximum deflection}

The deflection is generated by the bubbles and therefore the deflection should start at the moment or shortly after the bubble nucleation. Figure 5.15 shows the time at which the deflection starts as a function of the heating power. This same figure shows the data

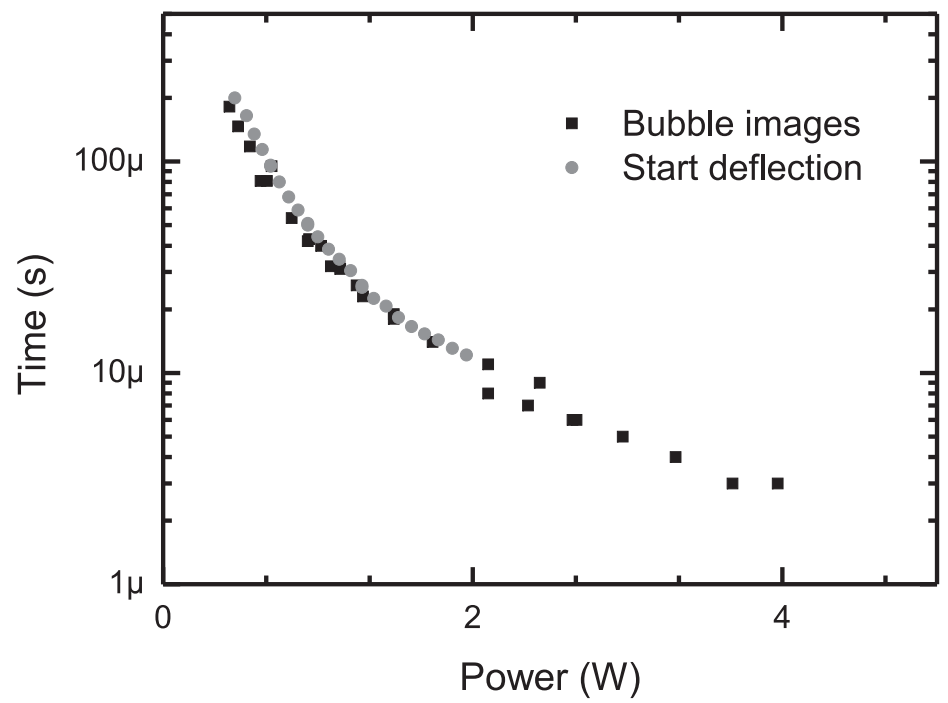

Figure 5.15: Measured onset of deflection of the membrane and bubble nucleation time for different applied powers.

from bubble imaging discussed in section 4.4.3. Both measurements were done under similar conditions. As expected the bubbles appear at the same time as the start of the membrane deflection. Small delays in time due to the distance between the heater and the membrane and other influences of the bubble dynamics, such as the effects of early nucleation discussed in section 4.5.3, are expected to be in the same range as the uncertainty of $1 \mu \mathrm{s}$ for the stroboscopic measurements. There influence can thus not be excluded or confirmed by these measurements.

The time of nucleation and thus the start of deflection should be independent of the length of heating pulse. The pulse only has to be long enough to ensure that the nucleation temperature is reached. For the previous measurements on the nucleation time, pulses much longer than the expected nucleation time were used to ensure that bubble nucleation takes place. Here the time at which the membranes starts its displacement as a function of applied heating power was measured for three different pulse lengths. As expected figure 5.16 looks similar to figure 5.15. The onset of deflection decreases with an increase in power. For a $10 \mu \mathrm{s}$ pulse a power of more than $1.4 \mathrm{~W}$ is needed to get a deflection. This power threshold increases to $2.0 \mathrm{~W}$ and $2.6 \mathrm{~W}$ for the $5 \mu \mathrm{s}$ and $3 \mu$ s heating pulses respectively. At powers below the threshold no considerable change in displacement could be detected, because no bubbles are generated. For the $10 \mu$ s pulse length the deflection starts $11 \mu \mathrm{s}$ after the heating pulse has started. At this moment the heating already has stopped and also for the $5 \mu \mathrm{s}$ and $3 \mu \mathrm{s}$ for the measurements at relatively low power the deflection starts after the heating pulse has stopped. In section 4.7.2 it was already shown that bubble nucleation can take place after the heating pulse. It, however, is also possible 


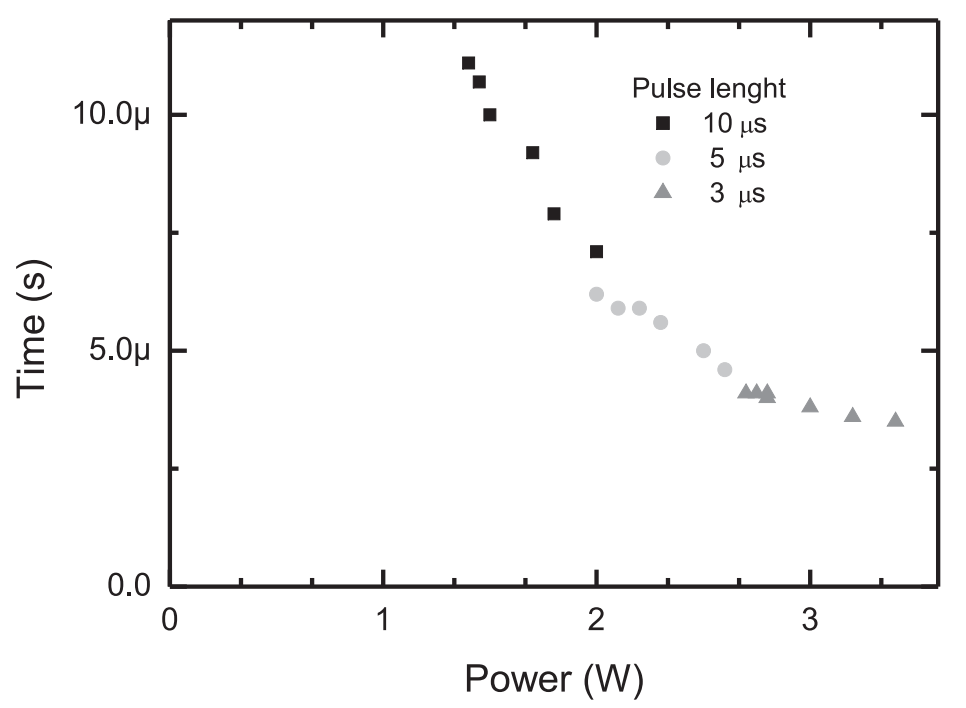

Figure 5.16: Start of deflection for three different pulse lengths.

that earlier discussed delay due to the distance between the bubble and the membrane is part of the cause. This delay is estimated at $0.5 \mu \mathrm{s}$, if a speed of sound in ethanol of $1144 \mathrm{~m} / \mathrm{s}$ and the cavity depth of $525 \mu \mathrm{m}$ are assumed. An estimation of the other delays, for example due to bubble dynamics, are more difficult to make. It can be concluded that for each pulse length there is a minimum power needed to get a considerable deflection and that this minimum power is directly related to the generation of bubbles.

One of the goals of the actuator was to have a deflection on the order of several micrometers within microseconds. The minimum power and thus the minimum energy to create a deflection is determined by the minimum energy to generate a bubble. The maximum deflection of the membrane will depend on the created bubble volume and the membrane properties. In the previous chapter it was shown that the applied heating power has a large influence on the bubble size and bubble geometry. The heating power will therefore also have a large influence on the maximum displacement. Figure 5.17 shows the maximum deflection at the centre of the membrane for three different pulse lengths as a function of heating power. For all the pulse lengths the dynamics are about the same. At low power no bubbles are generated and only a small deflection of a few nanometres due to the expansion of the ethanol near the heater is measured. If a power above a certain threshold is applied, the nucleation temperature will be reached and bubbles will be generated leading to a deflection of several micrometres. The largest deflections of nearly $6 \mu \mathrm{m}$ are reached for the $10 \mu \mathrm{s}$ pulse. The maximum deflection decreases with a decrease in pulse length, because a decrease in pulse length results in a smaller volume of heated liquid and thus a smaller maximum bubble size. A further increase in power above the threshold only leads to a slight increase of the maximum deflection for the $3 \mu \mathrm{s}$ and $5 \mu$ s pulses. This small increase can be related to an increase in maximum bubble volume. A minimum amount of energy is needed to generate the bubble. The extra energy due to the higher power increases the temperature of the liquid and the vapour in the bubble. This will lead to an increase of the maximum bubble volume. This extra volume, however, is relatively small in comparison to the volume change created by the formation of the 


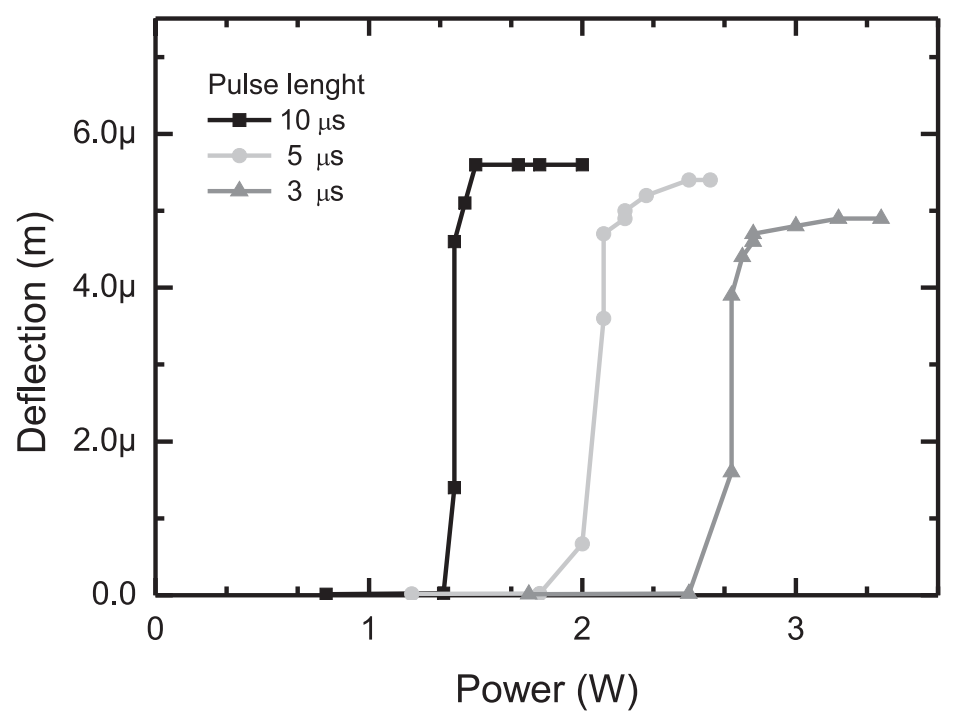

Figure 5.17: Maximum deflection for three different pulse lengths as a function of applied heating power.

bubble itself.

It takes a certain amount of time before the maximum deflection is reached after the onset of displacement. The total rise time can be used to calculate the speed of the initial deflection. The start of the deflection is shown in figure 5.16 and the moment of maximum deflection can also be easily determined from the displacement-time curve.

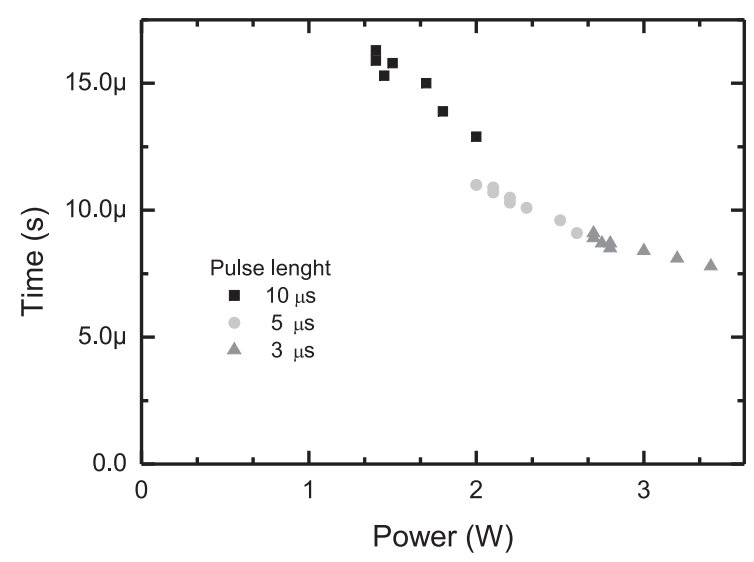

(a)

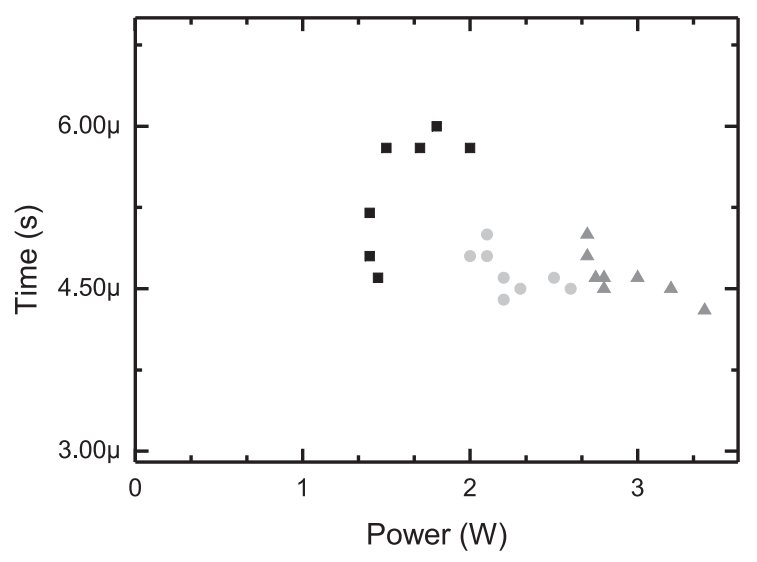

(b)

Figure 5.18: The time of maximum deflection (a) and the difference between the time of maximum deflection and the onset of deflection(b) for three different pulse lengths.

Figure 5.18a shows the time of maximum deflection as a function of heating power. Identical to the onset, the time of maximum deflection decreases with an increase in heating power. For these pulse lengths the maximum deflection is always reached after the heating power is turned off. Figure $5.18 \mathrm{~b}$ shows the difference in time between the onset of deflection and the maximum deflection. The calculated values lie between $6 \mu \mathrm{s}$ 
and $4.5 \mu \mathrm{s}$ and thus result in an average speed of $1 \mathrm{~m} / \mathrm{s}$. The initial deflection peak mainly shifts in time if the heating power is increased. The maximum displacement and the rise time hardly change with the applied heating power. A small decrease in maximum displacement is detected with a decrease in length of the heating pulse.

\subsubsection{Effect of the heater size and the membrane size}

The initial deflection is directly related to the volume of the generated bubble. The previous chapter has shown that the bubble volume depends on the heater size. The maximum deflection is therefore expected to change with the size of the heaters.

\section{Maximum deflection}

A number of samples with different heater sizes were examined at two different pulse lengths. Figure 5.19a shows the maximum deflection measured at heating powers just above the threshold power as a function of heater surface area. The size of the membrane for the samples was not identical for the different samples. Therefore the same data is shown as a function of the membrane area in figure 5.19b. The difference in deflection

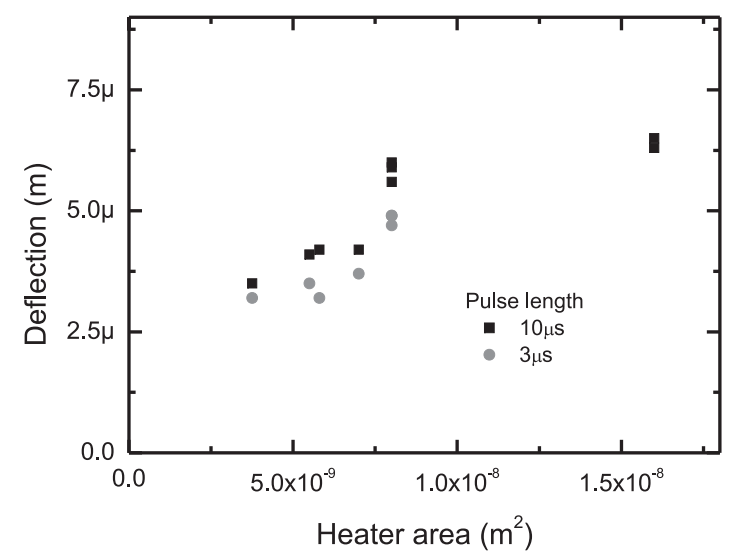

(a)

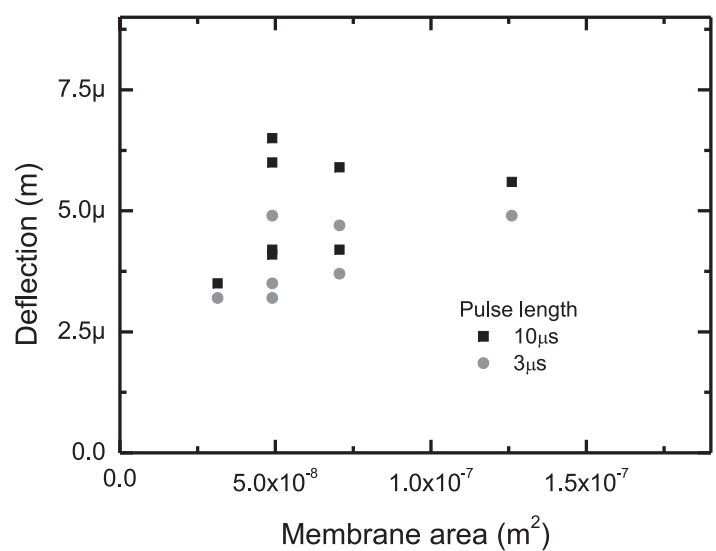

(b)

Figure 5.19: Maximum deflection as a function of heater area (a) and membrane area(b) for a $3 \mu \mathrm{s}$ and $10 \mu \mathrm{s}$ pulse length and a power just above the threshold power.

is relatively small in comparison to the range of the heater surface areas, but the $3 \mu \mathrm{s}$ as well as the $10 \mu \mathrm{s}$ pulse length the maximum deflection increases with surface area. The maximum deflection for the $10 \mu \mathrm{s}$ pulse is always larger than for the $3 \mu$ s pulse. No clear correspondence between the maximum deflection and the membrane area can be detected from figure 5.19b. Membranes with the same surface area show a large difference in maximum deflection.

\section{Volume of displacement}

Up to now only the deflection of the centre of the membrane was considered. If, however, a comparison between different membrane sizes must be made, it is much better to look 
at the total change in volume of the cavity due to the membrane deflection. The volume change at maximum deflection can be calculated from the deflection profile across the membrane diameter. Figure 5.20 shows two profiles for circular membranes of $200 \mu \mathrm{m}$ and $400 \mu \mathrm{m}$ diameter. The maximum deflection of both membranes differs only a couple of hundreds of nanometres, but the volume of displacement is much larger for the $400 \mu \mathrm{m}$ membrane.

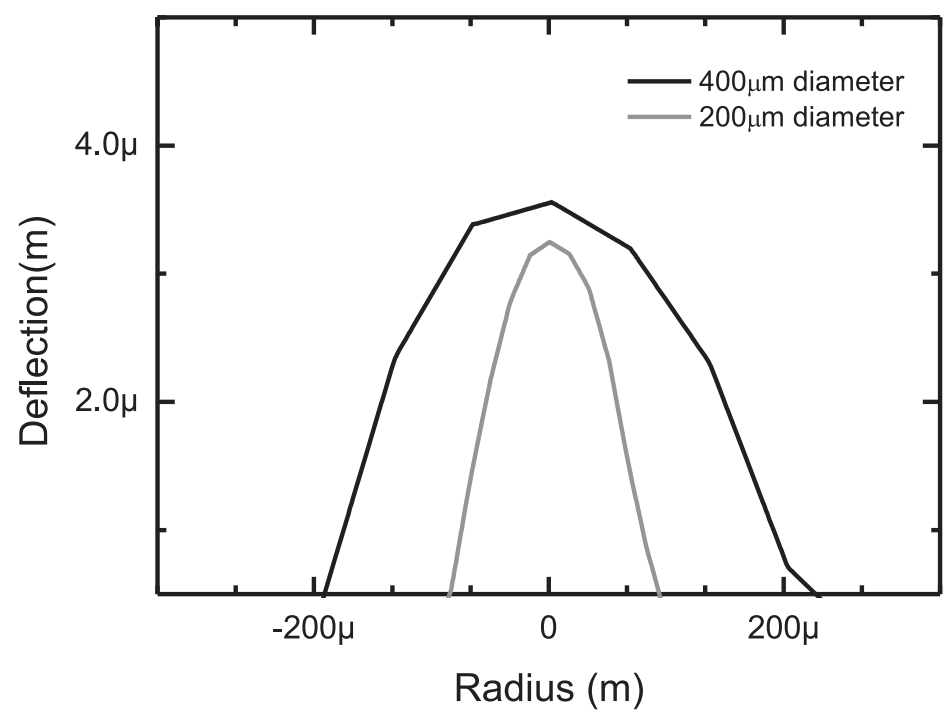

Figure 5.20: The profile of the deflection across the membrane diameter at the moment of maximum centre deflection for two different membranes.

The volume of displacement $(\Delta V)$ can be calculated by using equation 5.3

$$
\Delta V=2 \pi \int h(r) r d r
$$

where $h(r)$ is the deflection at a distance $r$ from the centre of the membrane. For all the measurements shown in figure 5.19 the profile at maximum deflection was determined and from this the total volume displacement was calculated. This volume is the volume change due to the dynamic displacement of the membrane generated by the bubbles. The change of the cavity volume due to a static pressure difference across the membrane can not be determined from the deflection profile determined with the laservibrometer.

The samples used in these measurements have different heater sizes as well as membrane sizes. To determine the influence of one of the two parameters the other parameter ideally should have the same value for the different samples. Unfortunately this was the case for only a small number of measurements. Figure 5.21a shows the calculated bubble volume as a function of heater area for four different samples, each with a $250 \mu \mathrm{m}$ diameter membrane. Although the number of data points is limited an increase in volume change for an increase in heater area can be detected. Figure 5.21b shows the change in volume as a function of membrane area for three different samples with a identical heater geometry and a heater area of $8.0 \times 10^{-9} \mathrm{~m}^{2}$. Due to the limited number of data points it can not be determined if there is a relation between the change of volume and the membrane area. 


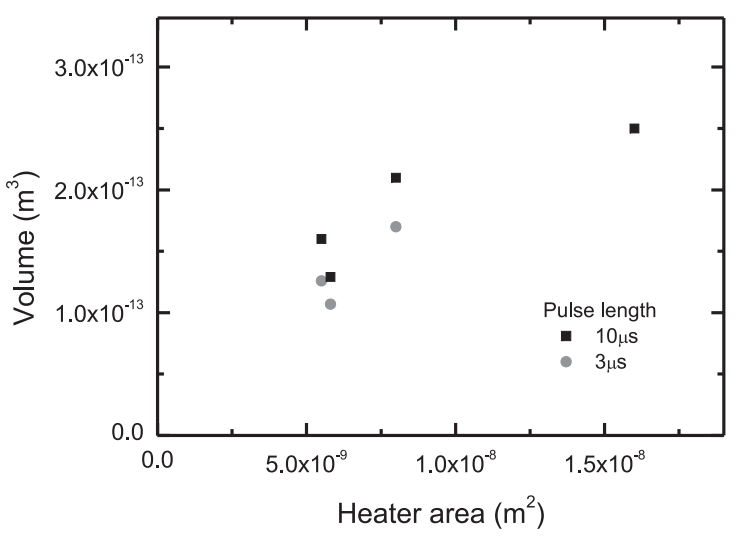

(a)

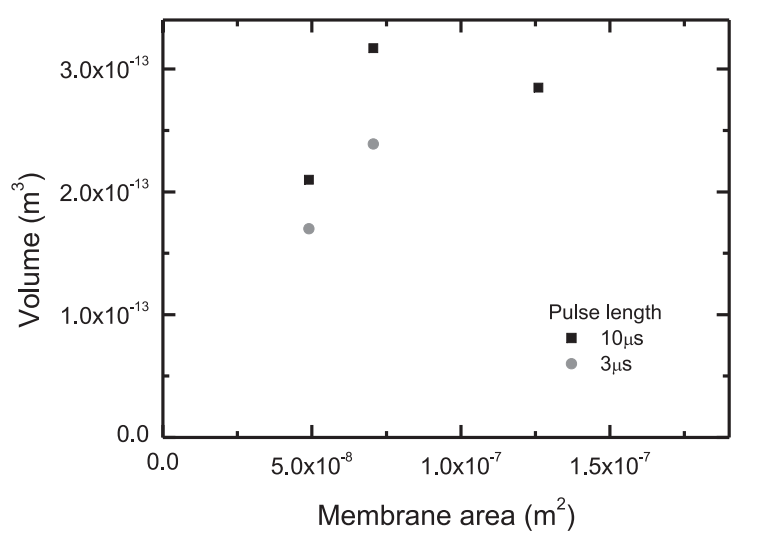

(b)

Figure 5.21: Calculated bubble volume as a function of heater area for a membranes with a $250 \mu \mathrm{m}$ diameter(a) and the calculated bubble volume as a function of membrane area for samples with a $8.0 \times 10^{-9} \mathrm{~m}^{2}$ heater area $(b)$.

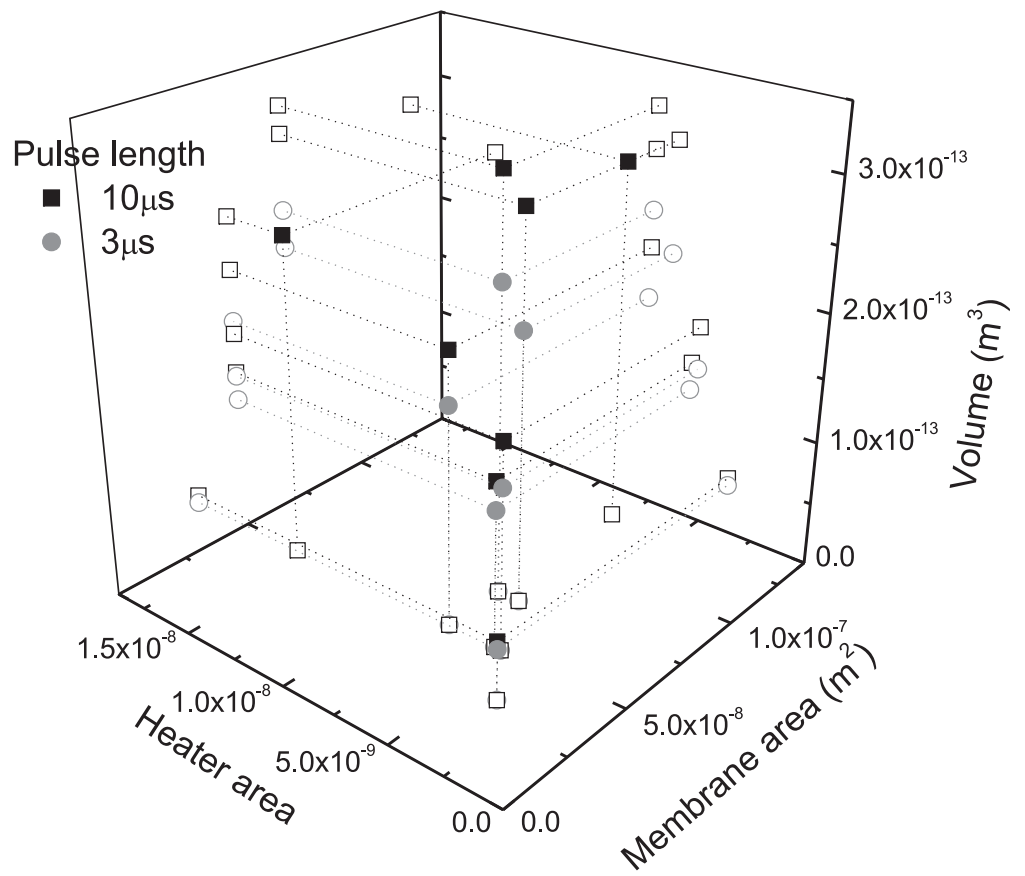

Figure 5.22: 3-Dimensional plot of the calculated volume of displacement as a function of heater area and membrane area for a $3 \mu \mathrm{s}$ and $10 \mu$ s pulse length. 
In order to compare the results found for the all the samples the data is plotted in a 3 -dimensional graph. Figure 5.22 shows a 3-dimensional plot of the volume change as a function of heater area and membrane area. This figure shows that for the majority of the samples the ratio between the heater area and membrane area has a value close to 10. In these cases the volume change increases linearly with the heater area or membrane area. Two samples have a considerably different ratio. For the sample with heater area of $1.6 \times 10^{-8} \mathrm{~m}^{2}$ and a membrane area of $4.9 \times 10^{-8} \mathrm{~m}^{2}$ this ratio is 3.3 and for the sample with a heater area of $8 \times 10^{-9} \mathrm{~m}^{2}$ and a membrane area of $1.3 \times 10^{-7} \mathrm{~m}^{2}$ the ratio is almost 16 . In both cases the change in volume at maximum deflection was smaller than expected from linear relation for device with a ratio close to 10 .

For an incompressible liquid the bubble volume and the volume change due to membrane displacement are identical. In chapter 4 it was shown the the bubble volume can be estimated by the product of the heater area and the height of the vapour sheet. As expected figure 5.21a and 5.22 show that the calculated volume change increases with the heater area.

The maximum volume change ranges between $4 \times 10^{-14} \mathrm{~m}^{3}$ to $3.2 \times 10^{-13} \mathrm{~m}^{3}$ Although Hong et al. [65] used water as the working liquid they found a bubble volume between $7 \times 10^{-14} \mathrm{~m}^{3}$ and $7 \times 10^{-15} \mathrm{~m}^{3}$ with heaters with a surface area of $2 \times 10^{-9} \mathrm{~m}^{2}$. Asai et al. [22] calculated the bubble volume created in an ink jet printer and found values between $1 \times 10^{-13} \mathrm{~m}^{3}$ and $5 \times 10^{-13} \mathrm{~m}^{3}$. The vapour volume in water calculated from the acoustic pressure measured by a pressure transducers by Zhao et al. [73] was between $4 \times 10^{-13} \mathrm{~m}^{3}$ and $3.0 \times 10^{-12} \mathrm{~m}^{3}$. Despite the use of ethanol as a working fluid, the values for the bubble volume, which were calculated from the volume change under the membrane, found in this research are comparable to the literature values.

No clear relation between the membrane size and the deflection can be found from figure 5.19b. This can only occur if the membrane is relatively flexible. The generation of a bubble creates a pressure impulse and the liquid above the heater will start to move. The moving liquid will only exert a force on the central part of the membrane. The pressure is thus not equally distributed across the membrane and the deflection profile will mainly determined by the geometry and volume of the created bubble. If the stiffness of the membrane has no influence at all, this would also imply that there should be no relation between the membrane area and the change of volume. Figure 5.22 however suggest that this relation does exist. The linear relation is mainly caused by the fact that in these measurements the membrane size is not an independent parameter, but has a nearly constant ratio of 10 with the heater size. Nevertheless, the results found for a few samples with ratio's different than 10 show that there is some influence of the membrane size. A decrease in membrane size will increase the stiffness. For relatively small membranes this increase in stiffness will result in smaller bubbles and therefore volume change will be smaller than expected. This effect is expected to be the reason that the sample with a ratio 3.3 shows a smaller volume than expected for its heater size. A closer look at the results shown in figure 5.22 reveals that the displaced volume of the sample with a $8.0 \times 10-9 \mathrm{~m}^{2}$ heater area and a $4.9 \times 10^{-8} \mathrm{~m}^{2}$ membrane area (ratio=6.1) is also relatively small in relation to its heater size and large in comparison to it membrane size.

On the other hand when the membrane is large and thus highly flexible the non- 


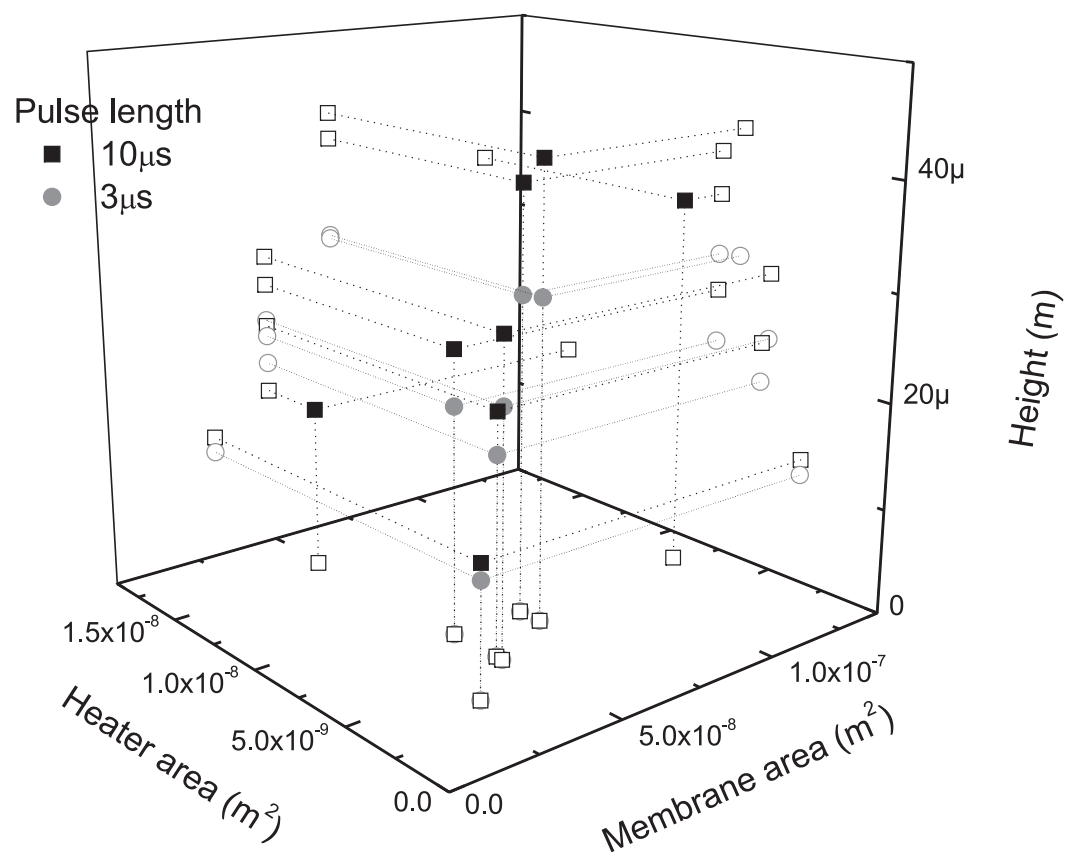

Figure 5.23: Height of the vapour bubble calculated from the volume change at maximum deflection.

circular geometry of the heater can have an effect on the measured membrane profile. The sample with a ratio of almost 16 has a heater in the form of a long strip. In this case the bubble will have a slightly rectangular shape and the deflection profile along its length will give a different calculated volume than the profile along its width. The value given in figure 5.22 was calculated from a profile along the heater width and this probably is the reason for the relatively small volume change for this particular sample. For most of the other samples the difference between heater length and width was much smaller and therefore this effect was less significant.

\section{Bubble height}

Under the assumption that the created bubble volume is equal to the volume change and that bubble has the form of a vapour sheet with an area equal the the heater surface, the bubble height can be estimated by dividing the volume change by the heater area. In figure 5.23 the calculated bubble height is shown as a function of heater area and membrane area. The previous chapter has shown that the bubbles will become rounded and become larger than the heater area as time progresses after the vapour sheet formation, especially for the $10 \mu \mathrm{s}$ pulse time. The calculations can thus only be used to get an estimate of the bubble height. A calculated height between $10 \mu \mathrm{m}$ and $45 \mu \mathrm{m}$ was found. This is comparable to the heights between $10 \mu \mathrm{m}$ to $70 \mu \mathrm{m}$ found from images in [71]. Varlamov et al. [50] show images of a bubble with a height of $40 \mu \mathrm{m}$ for bubble nucleation in ethanol under similar conditions. Asai et al. [56] found a bubble height between $70 \mu \mathrm{m}$ and $100 \mu \mathrm{m}$ 
for boiling in methanol.

For identical devices the bubble height measured for the $10 \mu$ s pulse time is always higher than the maximum height for the $3 \mu$ s pulse time. The height of the bubble increases with heater area as well as membrane area. Identical to the volume change a linear relation can be found for the samples with ratio 10 between membrane area and heater area. The samples with a different ratio deviate from this behaviour. The exact relation between bubble height and membrane or heater size is difficult to determine from these measurements.

\section{Energy and pressure impulse}

The maximum bubble volume can be related to the mechanical energy required to form the bubble and the pressure impulse generated during the formation of the bubble. The liquid is assumed incompressible and the maximum bubble volume is therefore equal to the volume displacement at maximum deflection. The work required to create a bubble in the liquid with a volume equal to the maximum bubble volume can be calculated from equation (2.27). At the maximum bubble volume the derivative of equation (2.27) with respect to time is equal to zero. The relation between maximum bubble volume $V_{\max }$ and the mechanical work $W$ used to overcome the cavity pressure and to increase the vapour/liquid interface surface area is

$$
V_{\text {max }} \approx \frac{W}{P_{a m b}-P_{s a t}\left(T_{a m b}\right)}
$$

For a highly flexible membrane the membrane stiffness can be neglected and therefore no mechanical energy is required to deflect the membrane. All the mechanical energy is utilized to create the maximum bubble volume and equal to the work calculated from 5.4.

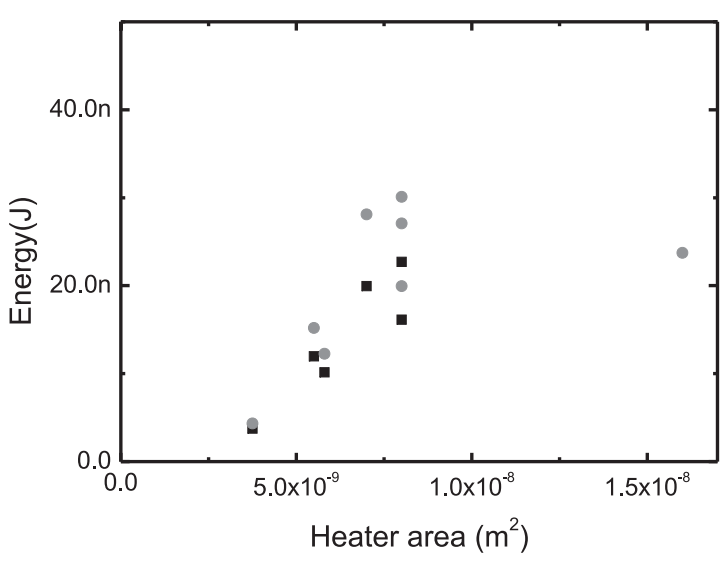

(a) Energy

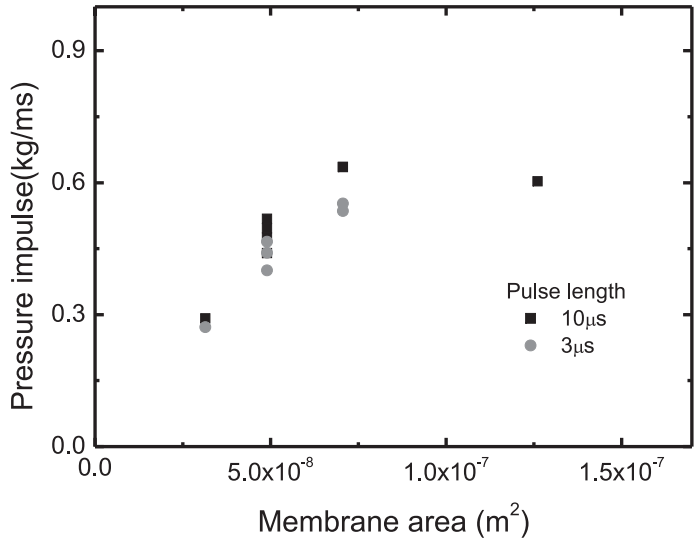

(b) Pressure impulse

Figure 5.24: Energy and pressure impulse used in the formation of the bubble calculated from the maximum volume of displacement.

Figure 5.24a shows the mechanical energy as a function of heater area. In the calculations the difference between the ambient pressure $\left(P_{a m b}\right)$ and the saturation pressure 
$\left(P_{\text {sat }}\right)$ were assumed to be equal to 0.95 bar for all the measurements. The energy increases with heater area from $4 \mathrm{~nJ}$ to $30 \mathrm{~nJ}$. The actual mechanical energy required to deflected the largest membrane was approximated at about $1 \mathrm{~nJ}$ for the largest membrane using Timoshenko et al. [85]. For the large membranes the energy required for membrane deflection is thus considerably smaller than the energy used for bubble formation and the approximation of a negligible mechanical energy required for the membrane deflection was valid. However for a smaller membrane more energy will be required and this energy cannot be neglected any more. For the smallest membrane the mechanical energy in the bubble and in the membrane will be comparable. The total mechanical energy, however is about a factor 1000 lower than the total energy input needed to create the bubble. A

large amount of energy is lost in the process. Such a low efficiency is typical for thermal actuators.

The magnitude of the pressure impulse $M_{p}$ required to generate bubble can be estimated from the mechanical energy using equation (2.30). In figure 5.24b the pressure impulse is shown as a function of the membrane size. The pressure impulse increases from $0.27 \mathrm{kgm}^{-1} \mathrm{~s}^{-1}$ to $0.64 \mathrm{kgm}^{-1} \mathrm{~s}^{-1}$. The difference in pressure impulse for different samples with identical membrane sizes is relatively small considering the large differences seen earlier in this section.

The membrane size or properties should have no influence on the generated pressure impulse. However in this case it was not the actual pressure impulse that was calculated, but the magnitude of the pressure impulse required to create a bubble volume equal to the displaced volume of liquid under the membrane. A part of the mechanical energy released during the formation of a bubble is used to deflect the membrane and a portion of the energy will be lost due to viscous effects and acoustic radiation. As the membrane decreases in size, the stiffness increases and larger part of the mechanical energy produced by the system is used to deflect the membrane. The maximum volume of the bubble as well as the volume displacement will therefore decrease in size.

\subsubsection{Effect of the pressure inside the cavity}

The pressure inside the cavity will change the bubble dynamics and change the static deflection. The change in static deflection will have an influence on the tension in the membrane. These changes will have a considerable influence on the initial deflection.

Figure 5.25 shows the maximum deflection as a function of the pressure difference across the membrane. In this measurement two different pulse lengths of $3 \mu \mathrm{s}$ and a $10 \mu \mathrm{s}$ with a repetition frequency of $1 \mathrm{hz}$ are used. The applied power is identical for all the data points of a particular pulse length. The maximum deflection decreases with an increase in cavity pressure. At low pressure the deflection for the $10 \mu$ s pulse is larger than for the $3 \mu \mathrm{s}$ pulse. As the pressure inside the cavity increases the difference in maximum deflection for the different pulse lengths decreases. For cavity pressures greater than 1 bar there is hardly any difference.

The static deflection is also shown in figure 5.25. The dynamic deflection is superimposed on this static deflection. At 0 bar pressure difference the maximum deflection will be nearly $6 \mu$. At 1.65 bar the maximum dynamic deflection will be $2 \mu \mathrm{m}$ and the static deflection is more than $5 \mu$, therefore the total deflection will be more than $7 \mu \mathrm{m}$. 


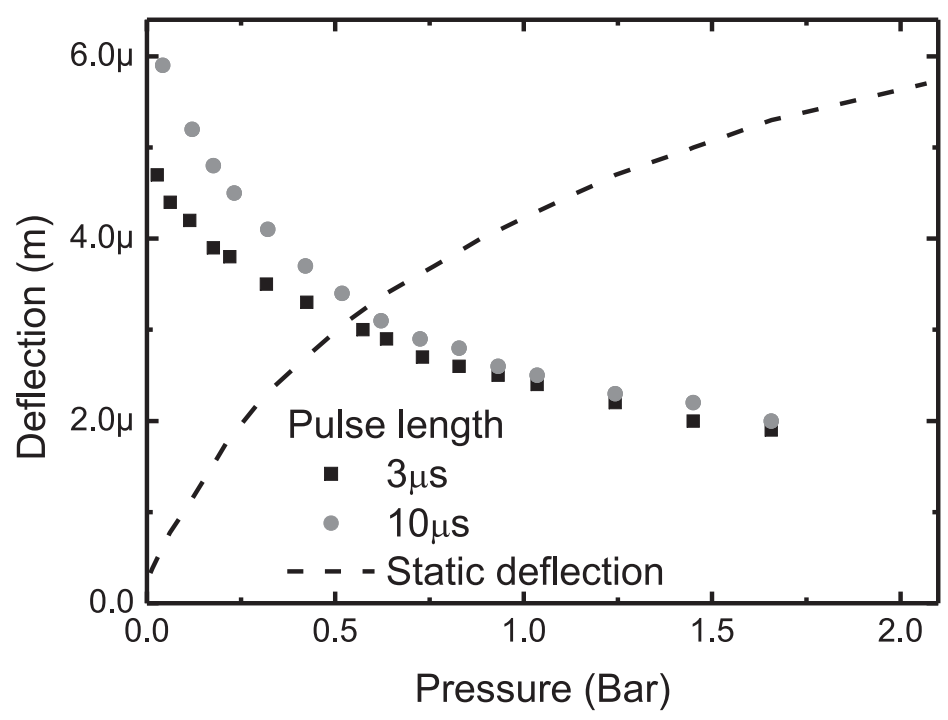

Figure 5.25: Maximum dynamic deflection for two different pulse lengths and the static deflection as function of pressure difference.

The increase in static pressure will change the tension in the membrane. Section 5.3.4 has shown that the application of pressure difference across the membrane will change the response of the membrane. The relative increase in stiffness of the membrane results in a smaller centre deflection and also the deflection profile at maximum deflection across the membrane will change with cavity pressure. Figure 5.26a shows the deflection profile for a $400 \mu \mathrm{m}$ diameter membrane at two different cavity pressures.

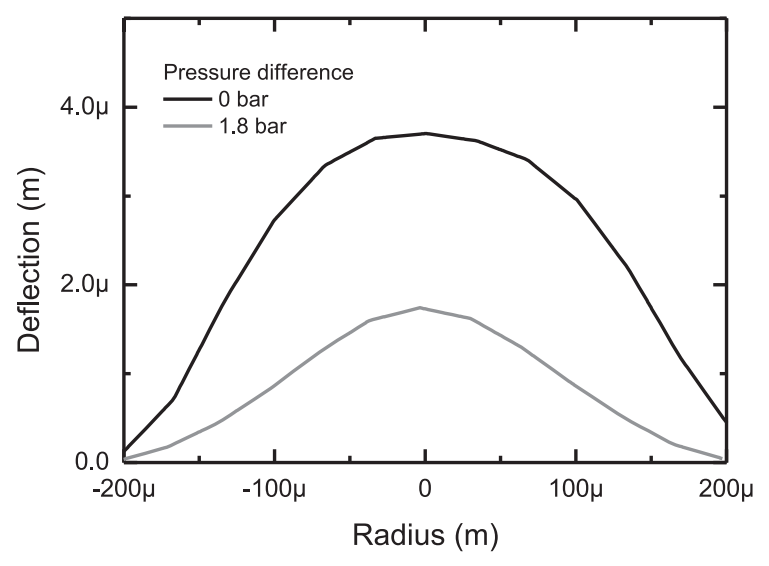

(a) profile

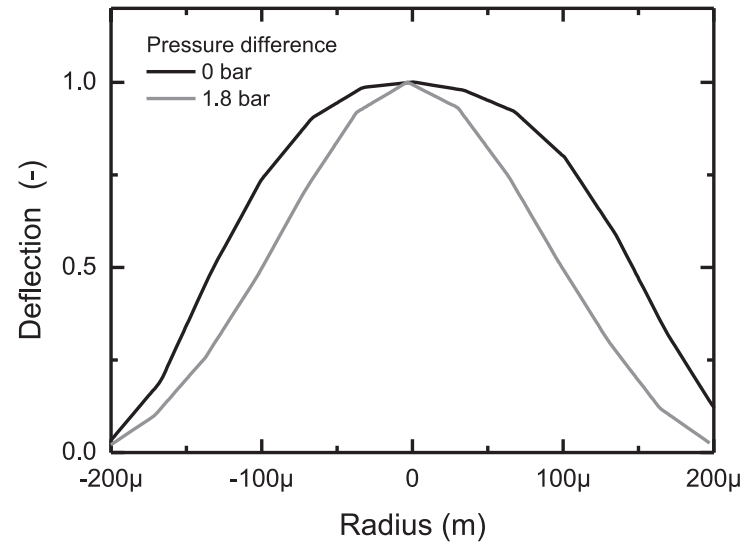

(b) normalize profile

Figure 5.26: Profile and normalized profile of the deflection across membrane diameter at the moment of maximum centre deflection for two different pressure differences across the membrane.

At higher pressure not only the maximum deflection is smaller, but also the deflection profile is different. This becomes more clear in figure 5.26b, where the normalized profiles of the membrane are shown at both pressures. At low pressure the curve of the deflection 
profile is relatively semi-circular, which is expected for a diaphragm that mostly bends. For the high pressure the curve shows more correspondence to a cosine function. This behaviour is characteristic for a stretching membrane. As the static pressure increases the dynamic deflection will be caused by stretching of the membrane and the influence of membrane bending will decrease.

Several parameters can be calculated from the membrane profile. Identical to the previous section the volume change under the membrane, the bubble height, the energy and the pressure impulse were calculated. The results are shown in table. This table also contains values found for a $10 \mu \mathrm{s}$ pulse and a heating power of $1.5 \mathrm{~W}$ for the same device.

\begin{tabular}{|c|c|c|c|c|c|c|}
\hline $\begin{array}{c}\text { Pressure } \\
(\mathrm{bar})\end{array}$ & $\begin{array}{c}\text { Pulse } \\
(\mu \mathrm{s})\end{array}$ & $\begin{array}{c}\text { Deflection } \\
(\mu \mathrm{m})\end{array}$ & $\begin{array}{c}\text { Volume } \\
\left(\mathrm{m}^{3}\right)\end{array}$ & $\begin{array}{c}\text { Height } \\
(\mu \mathrm{m})\end{array}$ & $\begin{array}{c}\text { Energy } \\
(\mathrm{nJ})\end{array}$ & $\begin{array}{c}\text { Impulse } \\
\left(\mathrm{kgm}^{-1} \mathrm{~s}^{-1}\right)\end{array}$ \\
\hline 1 & 3 & 3.7 & $2.3 \times 10^{-13}$ & 29 & 22 & 0.54 \\
1 & 10 & 5.5 & $3.1 \times 10^{-13}$ & 39 & 29 & 0.62 \\
2.7 & 3 & 1.7 & $7.0 \times 10^{-14}$ & 8.8 & 12 & 0.40 \\
2.8 & 10 & 1.8 & $8.1 \times 10^{-14}$ & 10 & 14 & 0.43 \\
\hline
\end{tabular}

Table 5.5: Different parameters calculated from the deflection profile at different cavity pressures and two different pulse lengths

The increase in cavity pressure results in a volume change and therefore a smaller bubble volume for both pulse lengths. The maximum height of the generated bubble at ambient pressure pressure is comparable to the heights calculated in the previous section. At increased pressure the height of the bubble is between 3 and 4 times smaller than at ambient pressure. The mechanical energy and pressure impulse used to create the bubble are also smaller at increased pressure. However, the total thermal energy introduced per heating pulse is independent of the cavity pressure and was $12 \mu \mathrm{J}$ and $14 \mu \mathrm{J}$ for the $3 \mu \mathrm{s}$ and $10 \mu \mathrm{s}$ pulse respectively. At high pressure an extra 10 to $15 \mathrm{~nJ}$ was used in the membrane deflection or in other processes. A simple approximation of the influence of cavity pressure can be made by calculating the difference in mechanical energy required to reach the total membrane deflection minus the energy required to reach the static deflection. The difference in mechanical energy for this membrane and at a pressure difference of 1.8 bar across the membrane is estimated at 10nJ. Although this is only a rough approximation, the energy is identical to the energy found from the measurements. Because the measurements were done on the same device the influence of static pressure on the pressure impulse, the mechanical energy and volume change was similar.

Identical to the previous section at ambient pressure the maximum centre deflection and maximum bubble volume created by a $10 \mu$ s pulse is larger than the bubble volume created by a $3 \mu \mathrm{s}$. Section 4.6 shows that at ambient cavity pressure the bubble geometry is more rounded and the area covered by the bubble is larger than at increased pressure. At relatively high pressures, however, there is only a small difference between the deflection and the volume change for two different pulse lengths. From images shown in section 4.6.4 it was determined that the bubble growth stops almost immediately after the vapour sheet is formed. The vapour sheet created by a $3 \mu$ s pulse is expected to be nearly equal to the sheet created by a $10 \mu \mathrm{s}$ pulse. Because the maximum deflection and created bubble 
volume are similar, the bubble height should also be nearly the same. At high pressure the influence of the pulse length is much smaller than at low pressures. At low pressure difference the influence of the membrane stiffness is much smaller than at high pressures differences. Although the total energy per pulse differs only slightly, the temperature distribution will be different for the different pulse lengths. The increase in stiffness and cavity pressure will decrease the influence of the temperature distribution in the liquid.

\subsection{Ideal response}

In the ideal actuator the heating pulse of several microseconds should generate a single deflection peak with a large displacement. After this peak the membrane quickly returns to its original state without any vibrations. In reality the response of the membrane was often quite different. Figure 5.9a shows a large number of vibrations after the initial deflection. These vibrations only show a small decrease in amplitude with an increase in time. In figure 5.7a the initial deflection is even smaller than the amplitude of the vibrations. The maximum deflection is reached at the fourth vibration peak. In this case the amplitude of the vibrations only show a small decrease with time. The response in figure 5.5 shows a closer correspondence to the ideal response. A large deflection peak of $6 \mu \mathrm{m}$ can be detected and after this peak the vibrations are dampened in $120 \mu \mathrm{s}$. The response which comes closest to the ideal response is shown in figure 5.27.

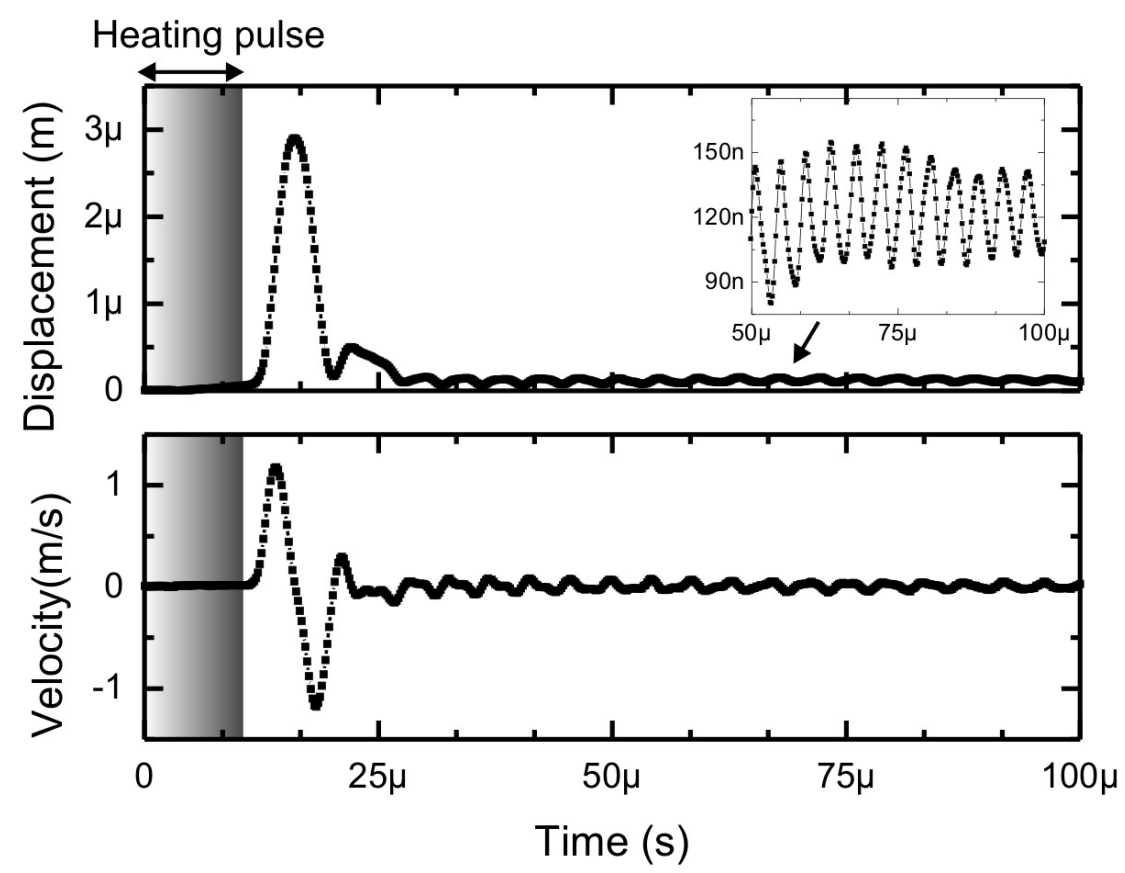

Figure 5.27: Velocity (bottom) and displacement (top) in time for a $1.9 \mathrm{~W}$ heating pulse measured with a scanning vibrometer. 
Figure 5.27 shows a measurement of a $250 \mu \mathrm{m}$ x $250 \mu \mathrm{m}$ square membrane for a $10 \mu \mathrm{s}$ heating pulse with a $100 \mathrm{~Hz}$ repetition frequency at an applied power of $1.9 \mathrm{~W}$. After $12 \mu \mathrm{s}$ the membrane starts to deflect and the membrane velocity increases to more than $1 \mathrm{~m} / \mathrm{s}$ at $14 \mu \mathrm{s}$. A maximum deflection of $3 \mu \mathrm{m}$ is reached within $16 \mu \mathrm{s}$, at this point the velocity of the membrane is $0 \mathrm{~m} / \mathrm{s}$. After this the membrane moves back reaching a downward speed of more than $1 \mathrm{~m} / \mathrm{s}$ at $18 \mu \mathrm{s}$. Twenty microseconds after the start of the heating pulse the membrane is again in its original position and only small decreasing oscillations around the normal position of the membrane could be detected. The total time of a single actuation, the heating and the deflection pulse, takes about $25 \mu \mathrm{s}$. The deflection peak is $3 \mu \mathrm{m}$ at its maximum and has width of $8 \mu \mathrm{s}$. In the previous section it was shown that the maximum height of the bubble will be between 10 and $45 \mu \mathrm{m}$, the average growth speed of the bubble will be between $2.5 \mathrm{~m} / \mathrm{s}$ and $12 \mathrm{~m} / \mathrm{s}$. This is comparable to the values of the speed of the bubble interface during growth determined from the images in the previous chapter. The velocity of the bubble interface is larger than the membrane speed for the growth and the collapse period.

The good performance of this actuator can be attributed to several causes. The membrane used here was the smallest square membrane. All the membranes used in this research have the same thickness. The stiffness of the membranes will increase for smaller membranes. This particular membrane was also coated with a thin metal layer. Therefore membrane is relatively thick and has a higher stiffness. This will have a large effect on the free-vibration; the amplitude will be lower and the natural frequencies will be higher. The persistence of the vibrations is relatively low.

For a square membrane only a few modes have a large centre deflection. The fundamental mode and modes with relatively large centre deflection like the $(3,1)$ will be excited the most by the initial deflection. The fundamental mode will always be highly dampened, but the damping of the $(3,1)$ mode will mainly determine the amplitude of the vibrations. For round membranes a much larger number of mode-shapes show a large centre deflection. All these mode-shapes will be excited and thus be present in the total vibration.

The ratio between the membrane area and heater area is relatively low and therefore the pressure generated by the vapour sheet will thus be divided equally over the membrane. This will result in an initial deflection profile which closely resembles the fundamental mode-shape. Smaller heaters, especially when they are placed out of centre, will result in an excitation of higher order asymmetric mode-shapes.

\subsection{Effect of the repetition frequency}

In the previous sections of this chapter only single pulses were considered. In this section the behaviour of the membrane at different repetition frequencies is investigated. Figure 5.28 shows the deflection as a function of the applied power at a repetition of $1 \mathrm{kHz}$. During these measurements the temperature at the start and at the end of the heating pulse were also monitored. Identical to the measurement for a single pulse (figure 5.17) a threshold power must be applied before a considerable deflection can be measured. Here, in contrast with the single pulse, an increase in power above this threshold leads to larger 


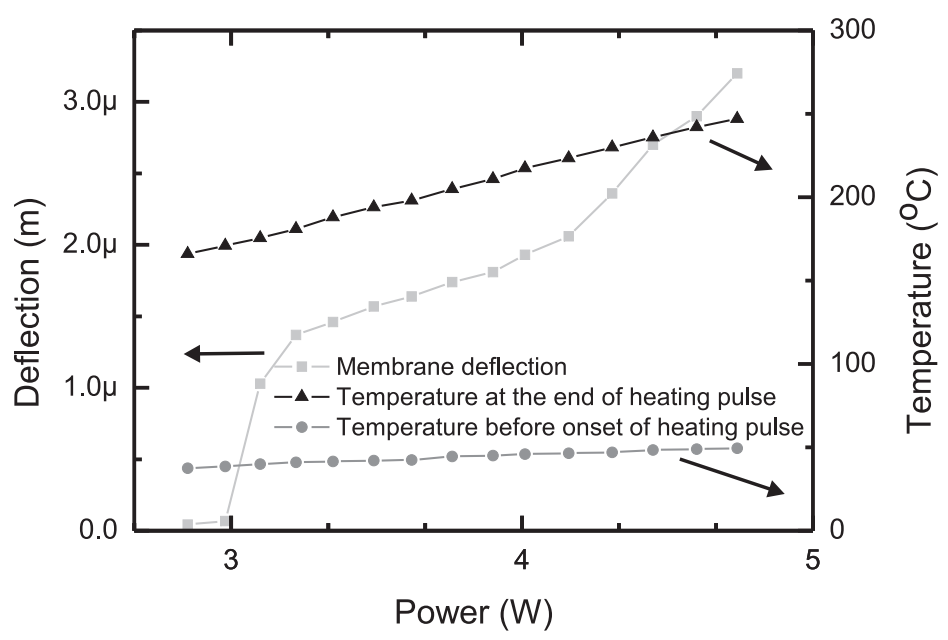

Figure 5.28: Membrane deflection and average heater temperature for a $3 \mu$ s pulse at a frequency of $1 \mathrm{kHz}$

deflection and above $4 \mathrm{~W}$ this increase extends. The increase in deflection can be related to an increase in energy input into the system. For a single pulse the system cools down to a temperature close to the ambient temperature. Here the temperature just before the onset of the heating pulse increases from $38^{\circ} \mathrm{C}$ at $3 \mathrm{~W}$ to $50^{\circ} \mathrm{C}$ at $4.75 \mathrm{~W}$. This increase in temperature leads to a larger volume of liquid with a temperature above the boiling temperature and will thus lead to larger bubbles. The bubble nucleation time decreases with an increase in heating power and the bubble has more time to grow as the applied power increases (section 5.4). This will result in a further increase in bubble size and this is most probably the cause of the extended increase of deflection above $4.75 \mathrm{~W}$.

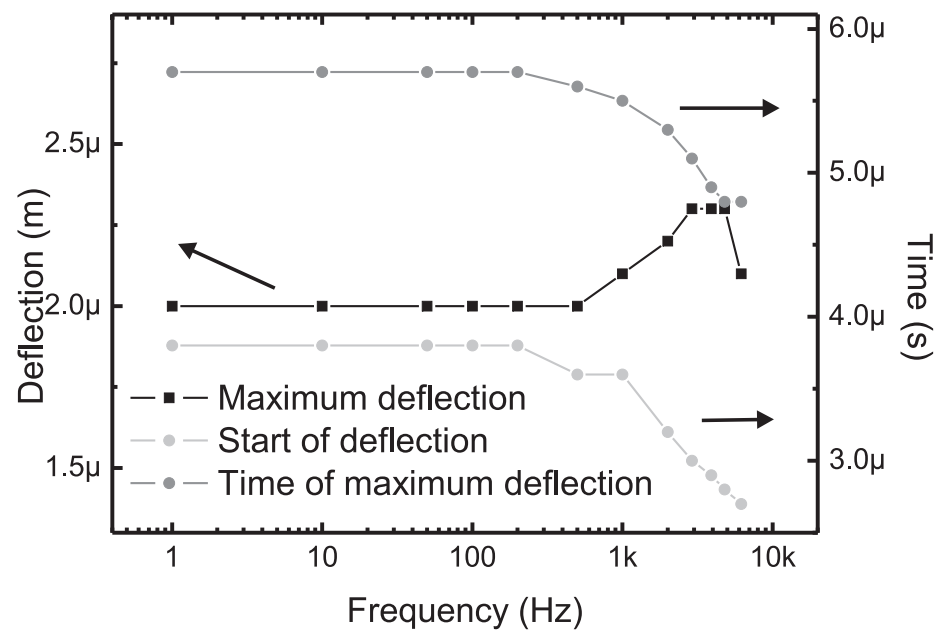

Figure 5.29: Maximum deflection, start of deflection and the time of maximum deflection for a $3 \mu$ s heating pulse of $3.0 \mathrm{~W}$

The effect of the repetition frequency on the average heater temperature and the bubble nucleation and growth at a increased repetition frequency were investigated in 
section 4.7. The membrane deflection will also change with the repetition frequency. Figure 5.29 shows the maximum membrane deflection, the start of deflection and time of maximum deflection as a function of the repetition frequency. All the measurements were done with for a $3 \mu \mathrm{s}$ heating pulse of $3.0 \mathrm{~W}$. Between 1 and $500 \mathrm{~Hz}$ the maximum deflection is nearly constant. Above $500 \mathrm{~Hz}$ the deflection is affected by the repetition frequency. First the deflection increases with frequency, but between $3.0 \mathrm{kHz}$ and $5.0 \mathrm{kHz}$ a maximum is reached. A further increase in repetition frequency results in a decrease in deflection.

Up to $200 \mathrm{~Hz}$ the increase in repetition frequency has no noticeable effect on the system. Above $200 \mathrm{~Hz}$ the temperature starts to rise and the nucleation temperature will be reached earlier. The bubble nucleation takes place earlier and therefore the deflection will start sooner after the start of the heating pulse. Above $500 \mathrm{~Hz}$ the increased temperature around the heater will result in larger bubbles and a thus a larger membrane displacement. Above $5 \mathrm{kHz}$ the deflection probably decreases due the formation of permanent bubble. The average temperature around the heater has risen to values where a permanent bubble can sustain it self during the cooling periods. This permanent bubble absorbs a large part of the pressure pulse generated by the explosive evaporation and this will lead to a decrease of the membrane displacement.

\subsubsection{Deflection control}

In the previous chapter it was shown that the power and energy needed to create a bubble decreases with frequency. If identical powers are used for low and high repetition frequencies the surplus energy at high frequencies at first results in a larger bubble. A further increase in repetition frequency will lead to the formation of a permanent bubble. At some point this will result in a decrease in the maximum deflection. In section 4.4.3 the minimum energy required to generate a bubble was investigated. Here the minimum energy to create a pre-determined deflection will be investigated. Figure 5.30 shows the applied heating energy and the temperature before onset of the heating pulse and at the end of the heating pulse as a function of frequency. The heating power decreases with repetition frequency from $3.1 \mu \mathrm{J}$ at $1 \mathrm{~Hz}$ to $2.6 \mu \mathrm{J}$ at $3.2 \mathrm{kHz}$. Over the same range the average heater temperature at the start of the heater pulse increases from $24^{\circ} \mathrm{C}$ to $43^{\circ} \mathrm{C}$. The temperature at the end of the heating pulse, although it fluctuates, stays around $210^{\circ} \mathrm{C}$.

Similar to the energy to create a bubble the minimum energy to create a certain deflection decreases with the repetition frequency. The average heater temperature at the end of the heating pulse is nearly constant despite the large difference in repetition frequency and heating power. Figure 5.28 already showed that there is a relation between the temperature at the end of the pulse and the deflection. A measurement of the heater temperature is less difficult than a measurement of the deflection and therefore the temperature could be used to determine and control the membrane deflection for a changing repetition frequency. In this case, unfortunately, a large uncertainty at relatively high temperatures prevents a determination of the exact relation between the final heater temperature and the maximum deflection. 


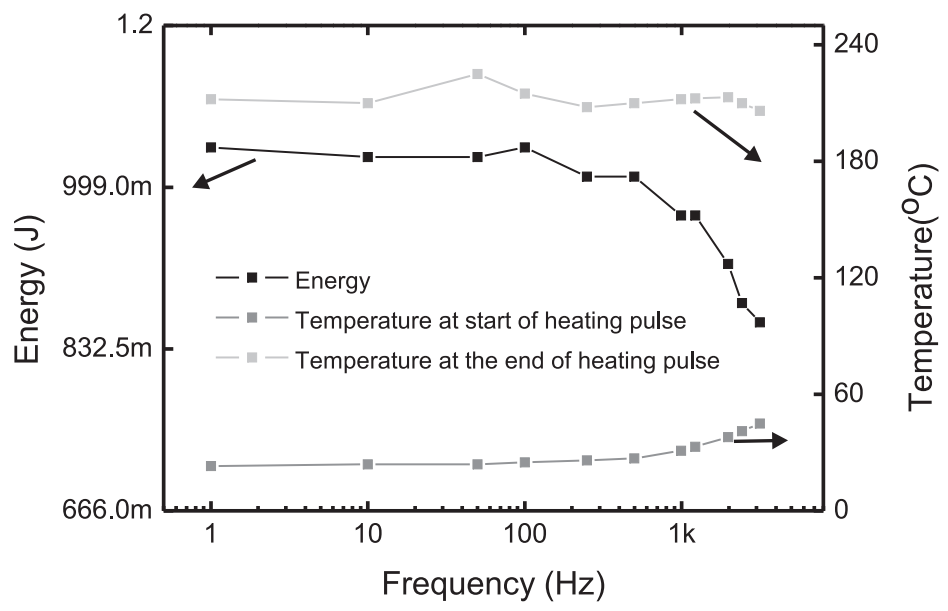

Figure 5.30: Minimum energy to create a pre-determined deflection of $3 \mu \mathrm{m}$ and the average heater temperature as a function of the repetition frequency.

\subsubsection{Influence of the cavity pressure at high repetition frequen- cies}

The effect of the cavity pressure on the bubble generation and the bubble dynamics was already discussed in chapter 4 . The effect of the cavity pressure on a single pulse was treated in section 5.4.3. Here the effect on the cavity pressure on the repetition frequency will be investigated. The deflection as a function of frequency was measured for three

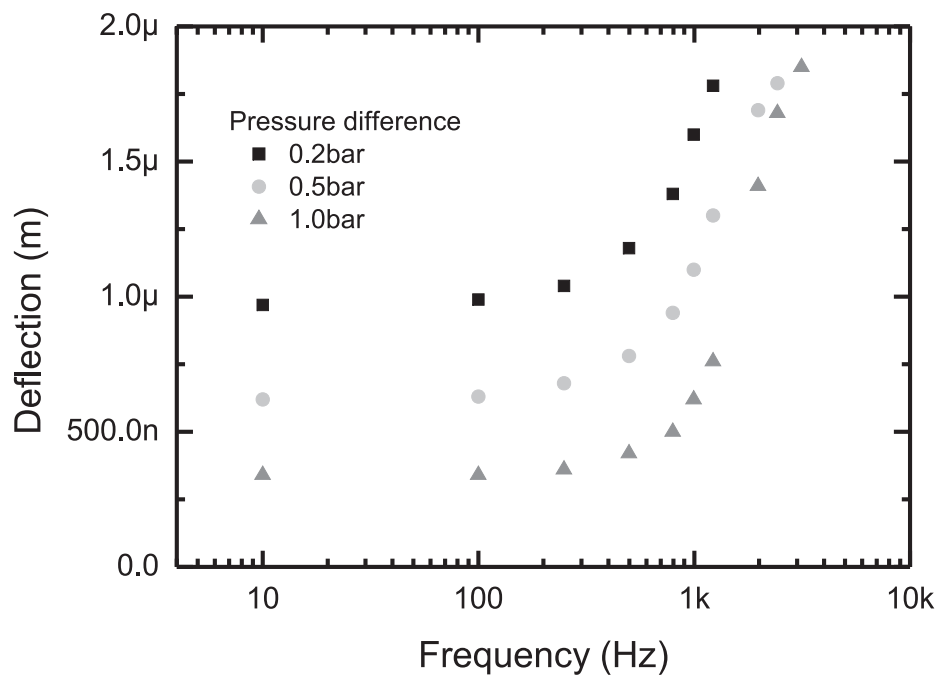

Figure 5.31: Membrane deflection for a $3 \mu$ heating pule and an applied heating power of $3.9 \mathrm{~W}$ at three pressure differences across the membrane.

different pressures inside the cavity. Figure 5.31 shows the results for a $3.9 \mathrm{~W}$ applied power and a pulse length of $3 \mu \mathrm{s}$. Similar to the behaviour shown in figure 5.29, the deflection increases with frequency for all the static pressure differences. The effect of an increase in pressure is identical to what was described in section 5.4.3, the deflection 
decreases for an increase in pressure difference across the membrane. Up to $1.2 \mathrm{kHz}$ the decrease in deflection with pressure is independent of repetition frequency. Above this repetition frequency the difference between the deflection at 0.5 bar and 1.0bar pressure difference decrease with repetition frequency. At the maximum repetition frequency the deflection is nearly equal for all the pressure differences. At frequencies close to the maximum repetition frequency a permanent gas-bubble will be present above the heater. The presence of a gas bubble in the system will lead to extra damping, but the exact influence of this bubble on the initial deflection and the vibrations is difficult to analyze.

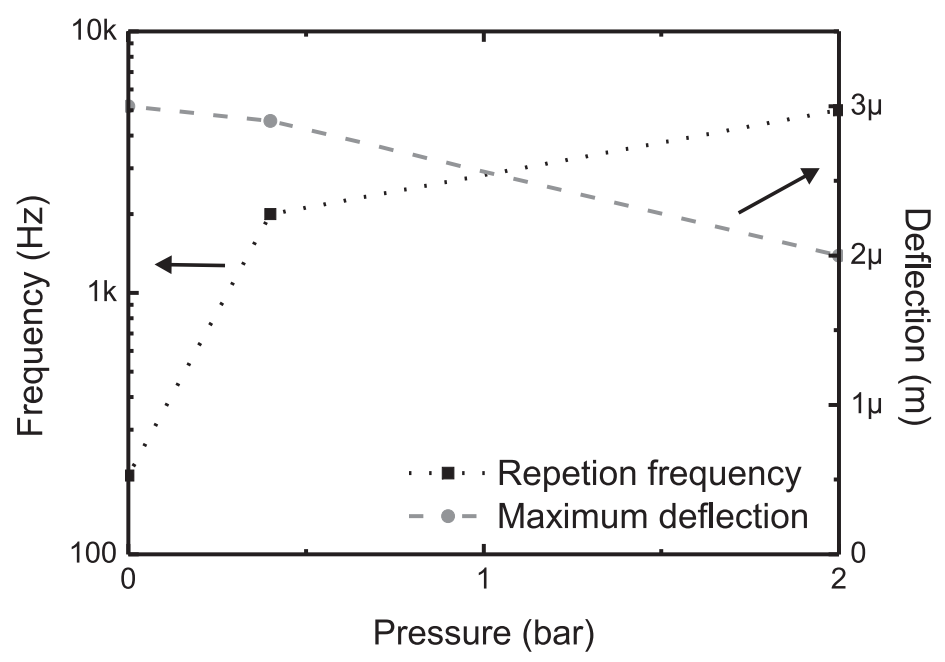

Figure 5.32: Maximum repetition frequency and maximum deflection at the centre of the membrane as a function of the pressure difference across the membrane.

The effect of cavity pressure on maximum repetition frequency and maximum deflection were also determined for a sample with a $200 \mu \mathrm{m}$ radius and a heater of $100 \times 50 \mu \mathrm{m}^{2}$. Figure 5.32 shows the maximum repetition frequency and the maximum deflection for a $3 \mu \mathrm{s}$ heating pulse at $1.8 \mathrm{~W}$. At ambient pressure the maximum repetition frequency is $200 \mathrm{~Hz}$. At a 2 bar pressure difference the maximum repetition frequency has increased to $5 \mathrm{kHz}$. The maximum deflection decreases from $3 \mu \mathrm{m}$ to $2 \mu \mathrm{m}$ over the same pressure range.

Although two completely different samples were used in the previous measurements the maximum repetition frequencies especially at the higher pressures differs only slightly. The maximum repetition frequency is reached at the moment the permanent bubble, formed above the heater, has reached a critical size and starts to influence the performance of the actuator. The size of the permanent bubble depends the pressure inside the cavity. An increase in pressure decreases the size of the gas bubbles and will also lead to a faster bubble collapse. In section 4.6.4 it was shown that the collapse time decreases considerably with an increase of the pressure difference across the membrane. The time between pulses can thus be decreased, if the cavity pressure is increased. This will automatically lead to an increase in repetition frequency. The short heating pulse at relatively low power ensures that the bubble growth stops almost immediately after the formation of the vapour sheet. The volume of the vapour sheet changes a little bit with pressure and a small change in deflection will occur. The maximum repetition frequency can thus be significantly 
increased by increasing the pressure inside the cavity. This increase pressure difference will only lead to relatively small decrease in maximum deflection for this sample.

\subsection{Summary and conclusions}

A constant pressure difference across the membrane will result in a static deflection. This static membrane deflection was measured with the white light interferometer. As expected the deflection increases with pressure and decreases with membrane size following the behaviour expected from theory. Circular and round membranes of comparable size show a similar deflection when subjected to a constant pressure. The pressure-deflection curves were used for membrane calibration and to calculate the Young's modulus and initial stress of the silicon nitride.

The dynamic deflection, generated by the explosive evaporation of ethanol and subsequent bubble growth and collapse, was measurement with a laservibrometer. The membrane is excited by the force generated by the bubble. The initial deflection can be seen as a superposition of all initial states of the membrane mode-shapes. After the initial deflection the bubble will collapse quickly and the membrane is left in free vibration. The total vibration is a superposition of the all mode-shapes oscillating at their own natural frequencies. The natural frequencies of the system are determined by the membrane and the liquid underneath. The bubble generation and the bubble dynamics have no significant influence on the natural frequencies. The natural frequency increases with a decrease in membrane size and with an increase in the pressure difference across the membrane. The mode-shapes are determined by the membrane geometry. For the circular as well as square membrane the profile of the deflection at the natural frequencies corresponds to a particular mode-shape. The amplitude of each mode-shape in the total vibration will change with time. Mode-shapes requiring a net-volume change will be highly dampened and will not show in the frequency spectrum.

The profile at maximum deflection, which is considered to be equal to the initial deflection from which the membrane starts to vibrate, shows a large centre deflection. Therefore, mode-shapes with a relatively large centre deflection, like the $(3,1)$ for a square membrane and the $(2,0)$ mode-shape for a circular membrane will have a relatively large amplitude in the frequency spectrum. These mode-shapes are also less affected by an increase in pressure. The initial deflection can be controlled by a number of parameters such as the energy of the heating pulse and pressure inside the cavity. The maximum deflection, which can be reached will also depend on the heater surface area and membrane size and properties. However, for the membrane sizes used in this research, the size of the membrane did not show a distinct effect on the generated maximum deflection. The diaphragms could still be considered as highly flexible membranes. At ambient pressure the energy needed for stretching or bending of the membrane is small in comparison to the mechanical energy generated by the formation and growth of a bubble.

The change of volume of the cavity due to the formation of a bubble is determined from the deflection profile across the membrane diameter. The effect of heater area and membrane area could not be determined independently, because for most devices the ratio between the two parameters was nearly the same. However, an increase of the heater area 
and the membrane area leads to an increase in the created volume change. The height of the bubble was estimated from this volume change and values between $10 \mu \mathrm{m}$ and $45 \mu \mathrm{m}$.

The area of the membrane was larger the heater surface area for all the devices. The maximum deflection of the membrane therefore will always be smaller than height of the bubble. The difference between deflection and bubble height will depend on the flexibility of the membrane, the distance between membrane and heater surface, and the ratio between heater surface and the membrane area. For the devices used in this research the membrane deflection was between 4 and 10 times smaller than the height of the vapour sheet.

The mechanical energy and pressure impulse generated by the formation of the bubble should only depend on the heating conditions. However the pressure impulse calculated from the volume displacement decreases with membrane size. As the membrane decreases in size it will become stiffer and a larger part of the impulse is needed to bend and/or stretch the membrane. The mechanical energy is divided between the bubble and the membrane. For the smaller membranes the stiffness of the membrane starts to influence the bubble volume and the membrane deflection. More mechanical energy is required to create a certain volume displacement. The difference in mechanical energy required to form the maximum bubble volume for several membrane sizes and at different cavity pressures can be used to estimated the performed work. This work could be used to perform useful work in an application. The influence on the centre deflection is more difficult to predict, because the deflection profile will also change with the membrane size.

The effect of a change in cavity pressure is twofold. A pressure difference across the membrane will create a permanent deflection and the distribution of the tension across the membrane will change. In general more energy will be required to generate a membrane deflection and the deflection profile will be different. An increase in cavity pressure also leads to decrease in the maximum bubble volume. A constant change in pressure difference across the membrane changes the profile of the dynamic deflection. At low pressure the dynamic deflection will be a combination of bending and stretching. For an increase in pressure stretching of the membrane becomes more important. At increased cavity pressure the influence of the pulse length and the temperature distribution close to the heater will be smaller.

The ideal response of the actuator to a single heating pulse is a single deflection peak of several microseconds with a large amplitude. The smallest square membrane had a response, which closely resembled the ideal behaviour. The good performance of this particular device can be explained by; the ratio between heater surface area and membrane area, the stiffness of the membrane and its square geometry.

The maximum repetition of the explosive bubble actuator is reached at the moment the permanent gas bubble formed at small distance above the heater starts to have a negative influence on the deflection of the membrane. This bubble is formed when the collapse time of a bubble is longer than the time between pulses. The size of this bubble will grow with system temperature and thus with repetition frequency. The maximum repetition frequency can be significantly increased by increasing the pressure inside the cavity. This increase in pressure difference will, however, lead to a small decrease in deflection. A maximum repetition frequency of $5 \mathrm{kHz}$ and a maximum deflection of $2 \mu \mathrm{m}$ were reached at a pressure difference of 2 bar. 


\section{Chapter 6}

\section{Conclusion and Outlook}

The main conclusions of the previous chapters are recapitulated in this chapter. In addition several improvements to the current design and possible applications are outlined. 


\subsection{Overall conclusion}

This research has two main goals. The first goal was to prove that explosive evaporation can be utilized to deflect a membrane and create an actuator, which is strong and fast. The second goal was to investigate the bubble nucleation and dynamics at high heat-flux conditions and provide useful data for future models.

The explosive micro-bubble actuator was fabricated using MEMS technology. Thinfilm heaters of platinum on a pyrex substrate form the bottom of a cavity etched in a silicon wafer. The top of the cavity sealed by a silicon-nitride membrane and the cavity is filled with a working liquid. A heating pulse of a few microseconds is used to generate a bubble. The formation of the bubble in this closed system creates a deflection of the membrane.

Information on the bubble nucleation and bubble dynamics inside the bubble actuator was gained from images taken by stroboscopic imaging and by monitoring the heater temperature during the heating pulse. The moment of bubble nucleation can be determined from images taken by stroboscopic imaging as well as from the temperature response. Two different regimes of nucleation are distinguished. For relatively low heatfluxes $\left(100 \mathrm{MW} / \mathrm{m}^{2}\right)$ the nucleation of a single bubble takes place at more than $50 \mu$ s after the start of the heating pulse. The micro-bubble actuator will use much shorter pulses with a much higher heat-flux. For nucleation times below $25 \mu \mathrm{s}$ most of the bubbles are generated by spontaneous nucleation. At a temperature of $185^{\circ} \mathrm{C}$ a large number of bubbles formed on the heater instantly coalesce into vapour sheet.

The bubble dynamics can be divided in three stages. In the growth stage bubbles are generated and quickly grow to a maximum bubble size. The intermediate stage is characterized by alternating collapse and growth periods. In the final stage the bubble decreases in size until it finally collapses completely. The start of the final stage is mainly determined by the total pulse length, but can start long after the heating pulse has stopped. All the stages are influenced by the energy input per pulse. In general all the events, such as bubble nucleation, maximum bubble volume and bubble collapse take place earlier if the energy is increased. The amount of energy needed to get a nucleation decreases with the pulse length, but the bubbles generated by these pulses have a smaller volume. The intermediate and final stage are also influenced by the pressure inside the cavity. The bubble volume will decrease with pressure and the collapse speed of both vapour and gas bubbles will increase with pressure. The collapse time will thus considerably decrease with an increase in cavity pressure.

The stroke generated by the actuator is equal to the centre deflection at maximum bubble volume in most cases. The displacement and speed of the membrane caused by the bubbles were determined in real-time. The centre of the membrane shows a maximum deflection between $2 \mu \mathrm{m}$ and $6 \mu \mathrm{m}$ and a deflection speed of more than $1 \mathrm{~m} / \mathrm{s}$ was measured with a laservibrometer. The ethanol liquid and vapour are assumed incompressible and therefore the change in cavity volume due to the membrane displacement is equal to the created bubble volume. From the stroboscopic images it was determined that the bubble is a thin vapour sheet. The volume of this sheet can be estimated by taking the product of the surface area of the heater and the height of the vapour sheet.

The height of the vapour sheet depends on parameters such as the energy input per 
pulse, the cavity pressure and the repetition frequency. This height could not be determined from the images, but can be calculated from the volume displacement of the membrane. For the short heating pulses used here this height is estimated at several tens of micrometers and the values found here are comparable to values found in literature. In the current design the membrane is always larger in surface area than the heater area, therefore the deflection will always be smaller than the bubble height. The difference between bubble height and centre deflection will mainly depend on the ratio between membrane size and heater size, and the tension in the membrane.

The bubble volume is highly dependent on surface area of the heater, while the geometry of the heater has no significant effect on the volume of the vapour sheet. However at high heat-fluxes the heater design can become important. A non-uniform heat-flux across the heater during the heating pulse can result in a much higher current density at some parts of the heater, in particular in sharp corners. The heat-flux at these points will be higher and the nucleation temperature of the liquid will be reached earlier. This will lead to an earlier nucleation at those spots. Although nucleation on the rest of the heater will occur if heating is continued, this effect is unwanted. Simulations of the current density can predict the sites and the chance of early nucleation. The simulations can therefore be used to optimize the heater design.

In this research it was the first time that bubbles formed by explosive boiling in a closed cavity with flexible membrane on one side were studied. The flexibility of the membrane will determine the influence of the membrane on the bubble generation and bubble dynamics. Several different samples with different membrane sizes were fabricated and studied. In absence of a permanent pressure difference across the membrane most of membranes are relatively flexible. The energy used in the bubble growth process is much larger than the energy needed to stretch or bend the membrane. An increase in pressure difference across a membrane or a decrease in membrane size will increase the stiffness of the membrane. More energy is needed to create a deflection and the effect membrane stretching will increase its importance with respect to bending of the membrane. This will also change the deflection profile at maximum displacement.

After the initial deflection, which is a superposition of all the excited mode-shapes, the system will be in dampened free-vibration. The amplitude and damping of the different mode-shapes depend on size, geometry and properties of the membrane. Mode-shapes with a large centre deflection are excited the most. Mode-shapes with a net-volume change are heavily dampened. The stiffness of the membrane can be increased by an increase in pressure difference across the membrane. This will result in an increase of the natural frequencies and a decrease in amplitude of the mode-shapes.

The operation speed of most conventional thermal actuators is low due to the slow heat-diffusion processes. In the bubble actuator the speed of bubble growth and collapse of vapour are several tens of $\mathrm{m} / \mathrm{s}$ and it takes only a few microseconds to create a bubble. Therefore a vapour bubble can be created and completely collapse within $20 \mu \mathrm{s}$. The heating conditions, the heater size and the heat-transfer will determine how long it will take the system to cool-down. Especially at higher repetition frequencies the system will start to heat-up, but in theory repetition frequencies up to $50 \mathrm{kHz}$ should be possible. However, if only a small amount of gas is dissolved in the liquid, the bubble formed by explosive evaporation will also contain some gas. The residual gas has to dissolve into the 
liquid. This process is much slower than condensation of vapour and is determined by the diffusion speed of the specific gas through the liquid. The amount of gas inside the bubble will therefore determine the total collapse time. The maximum repetition frequency of the actuator is reached at the moment that a permanent bubble is formed above the heater surface and starts to have a negative influence on the actuator performance. This permanent bubble is formed when the time between heating pulses is shorter than the time of bubble collapse and will contain vapour as well as gas. The size of this bubble will depend on the temperature of the liquid and the pressure inside the cavity. After the heating stops the bubble will quickly decrease in size due to condensation of the vapour and the concentration of residual gas in the bubble will increase. After most of the vapour in the bubble has condensed, the speed of decrease in bubble volume is mainly determined by the dissolution of the gas in the liquid.

The maximum repetition frequency is highly dependent on the amount of residual gas in the liquid. Degassing the liquid is therefore very important. A low concentration of gas will result in smaller residual bubbles and a faster dissolution of the gas in the bubble. It is almost impossible to degas a liquid completely and there will always be a small amount of gas present in the liquid. An increase in cavity pressure will lead to a change in the solubility of the gasses and therefore a faster bubble collapse. Because the increase in pressure difference across the membrane only results to a small decrease in maximum deflection the increase in cavity pressure is an effective way to increase the operating frequency of the actuator.

The best performance up this point was a maximum repetition frequency of $5 \mathrm{kHz}$ with a deflection of $2 \mu \mathrm{m}$ at the centre of the membrane at a pressure difference of $2 \mathrm{bar}$ across the membrane. At low repetition frequency and ambient pressure inside the cavity the maximum dynamic deflection at the centre of the membrane was $6 \mu \mathrm{m}$ and maximum displacement speed more than $1.3 \mathrm{~m} / \mathrm{s}$ was measured. The maximum volume displacement was $3.2 \times 10^{5} \mu \mathrm{m}^{3}$ and this is equal to the maximum bubble volume. From this the maximum bubble height was estimated at $45 \mu \mathrm{m}$ and the bubble growth speed at $12 \mathrm{~m} / \mathrm{s}$. A change in the ratio between membrane area and heater area should make it possible to increase the maximum deflection to a deflection equal to the bubble height. However,in order to reach these large deflections, the energy required for the membrane deflection should still be negligible.

The total energy required to generate a bubble is several micro Joules. Only a fraction of this thermal energy is converted to mechanical energy. Such a low efficiency is typical for thermal actuators, but because the energy density is high, the pressure inside the bubble is 25 bar directly after bubble nucleation. At increased cavity pressures and for small membranes a significant part of the generated pressure impulse, a maximum of 0.3 $\mathrm{kgm}^{-1} \mathrm{~s}^{-1}$ for the devices used in this research, is utilized to deflect the membrane. At ambient pressure about $29 \mathrm{~nJ}$ of mechanical energy is used to form the bubble and less than $1 \mathrm{~nJ}$ is required to create the maximum membrane deflection for the larger membranes. However, at a cavity pressure of 2.8bar the maximum bubble volume is smaller and only $14 \mathrm{~nJ}$ is used for bubble growth, the remaining $15 \mathrm{~nJ}$ is utilized to create a membrane deflection. This proves that the mechanical energy generated by the formation of a bubble can produce useful work. Section 6.3 will give some examples how this work can be used in applications. 


\subsection{Design improvements}

The micro-bubble actuator can generate deflections of several micrometers at frequencies of more than a kilohertz. Still a number of modifications can be made to the design to improve the performance of the actuator. Some of these changes will be easy to implement. Other alterations will be more difficult and require radical changes in the design, the fabrication or the liquid handling. For a number of modifications mentioned below, the precise effect on the performance can not predicted easily and further investigations will be necessary to determine the outcome. Here simulations can be a useful tool and the results of experiments can be used to check the outcome of the simulations. The improvements can be divided in three areas:

- Heaters and substrate

- Working liquid

- Membrane

\subsubsection{Heaters and substrate}

The current design of the actuator is relatively simple and there are only a few critical steps in the fabrication process. A change in the design of the heaters or the use of a different substrate material can lead to a considerable improvement in the performance. However, in most cases these changes will result in a more complex design and an increase in the number of fabrication steps. A few relatively simple changes to the design of the heaters and substrate are suggested below.

\section{Suspended heaters}

A large part of the heat produced by the heaters is "lost" in the substrate. There are several solutions to improve the efficiency. If the heaters are suspended in the liquid, the complete heater will be in contact with the liquid and nearly all the energy produced by the heater will be transferred to the liquid. However, a heater completely surrounded by liquid can also have a disadvantage. After the heating pulse the liquid has to cool down. For the design used in this research the heat spreads more slowly in the liquid than in the substrate (section 2.2). The substrates therefore improves the cooling of the liquid after the heat pulse. In a heater completely surrounded by liquid only the liquid will determine the cooling speed.

Furthermore a suspended heater will be exposed to the forces generated during the bubble collapse. It is well known that the collapsing bubbles can lead to cavitation damage. The heater should be strong enough to withstand these cavitation forces. In this design the heaters are very thin and would be too fragile to be suspended in the liquid. An increase in thickness of the metal or placing the heater on a supporting structure should make a suspended heater possible. 


\section{Heater on the membrane}

The placement of the heater on the membrane will have two effects. The membrane is relatively thin and has air on its other side. The conductivity of heat through the membrane into the air will be relatively low. A larger part of the heat produced by the heater will therefore be transferred to the liquid and this will improve the efficiency. Secondly the bubbles produced by explosive evaporation will not only exert a pressure on the liquid, but will also exert a pressure directly on the heater and the membrane. This might lead to a larger deflection, because the effects of liquid compression and other sources of energy dissipation will be smaller. Placing the heater on the membrane, however, requires a flexible heater. The membrane will also be exposed to the cavitation forces if the heater is placed on the membrane. Designing a membrane with a flexible heater for these circumstances is a considerable challenge.

\section{High conductivity substrate and insulation layer}

The use of a substrate with a high conductivity will improve the cooling after the heating pulse. However, more energy will be needed to create a bubble, because a larger part of the total power will be transferred to the substrate. If a thin layer with a low thermal conductivity is placed in between the liquid and the substrate, this layer will act as a thermal insulation during the short heating pulse. In order to work properly the layer should be thicker than the heat penetration depth of the heating pulse. After the pulse has stopped and the system will start to cool down, the heat is transferred mostly through the high conductivity substrate. The effect of the insulation layer during the relatively long cooling time is small. During the heating pulse a considerable part of the produced thermal energy will be transferred to the liquid, but after the heating pulse the thermal energy is quickly removed from the system through the high conductivity substrate.

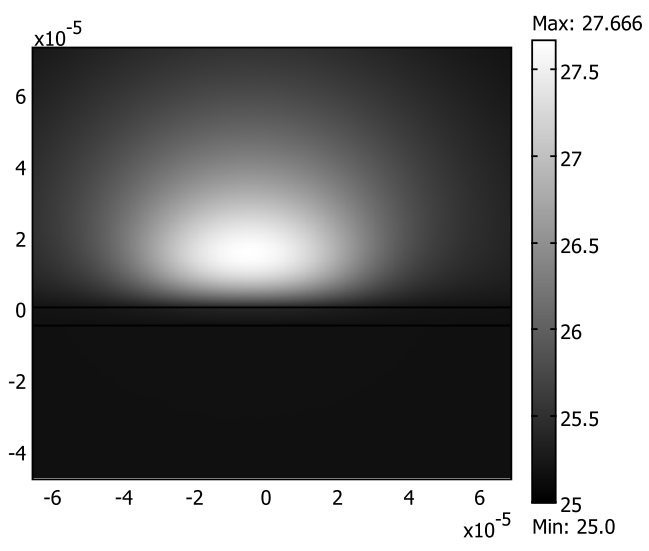

(a) Temperature profile

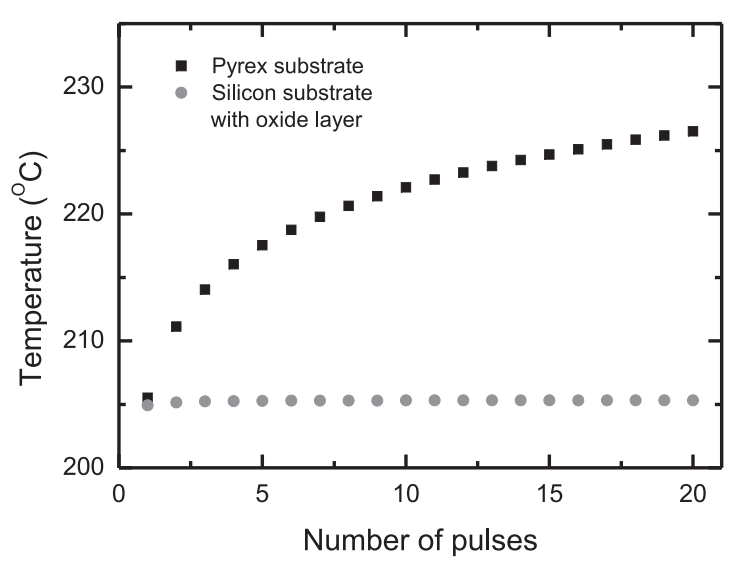

(b) Heater temperature

Figure 6.1: Temperature profile $(y-z)$ at the end of a cooling period after 20 pulses for a silicon substrate with a $5 \mu \mathrm{m}$ silicon-oxide insulation layer (a). The heater temperature at the end of the heating pulse for the pyrex substrate and the silicon substrate with an insulation layer as a function of the number of pulses (b). 
Simulations identical to the simulations described in section 2.2.3 were carried out, but instead of a pyrex substrate, a silicon substrate with a $5 \mu \mathrm{m}$ layer of silicon-oxide between the heaters and the substrate was used. Figure $6.1 \mathrm{~b}$ shows the heater temperature at the end of the heating pulse as a function of the number of pulses. For the silicon substrate with an insulation layer the temperature after the first pulse is lower and slightly more energy will be required to reach the nuclation temperature. The temperature increase with the number of pulses, however, is much smaller than for the pyrex substrate. This already shows that the silicon substrate does not heat up as fast as a sample with a pyrex substrate. Figure 6.1a shows the temperature at the end of the cooling period after 20 heating pulses. If this profile is compared to figure $2.13 \mathrm{a}$, it shows that the entire silicon substrate has a temperature close to the ambient temperature and only a part of the liquid has an elevated temperature. The silicon substrate does not heat up as much as the pyrex substrate due to heating pulses and the heat is quickly distributed throughout the entire substrate. Identical to the sample with a pyrex substrate (figure 2.13a) the highest temperature can be found in the liquid at some distance from the heater surface. The heater temperature as well as the maximum temperature found in the liquid, however, are much lower than for the pyrex substrate (figure 6.2a). The silicon substrate with the insulation shows a considerably smaller increase in the temperature with the number of pulses. After only 5 pulses the temperature of heater and the maximum liquid temperature at the end of the cooling period are nearly constant. Figure $6.2 \mathrm{~b}$ shows that the distance between the heater surface and the point in the liquid with the highest temperature is much larger for the silicon substrate.

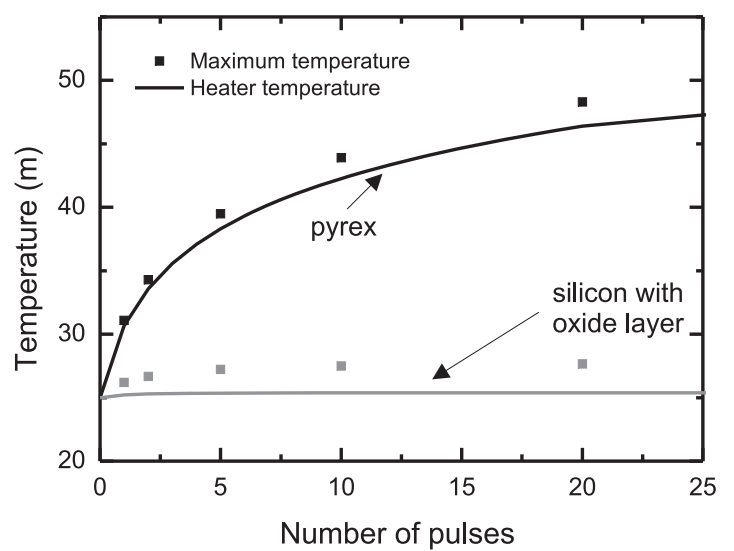

(a) Temperature

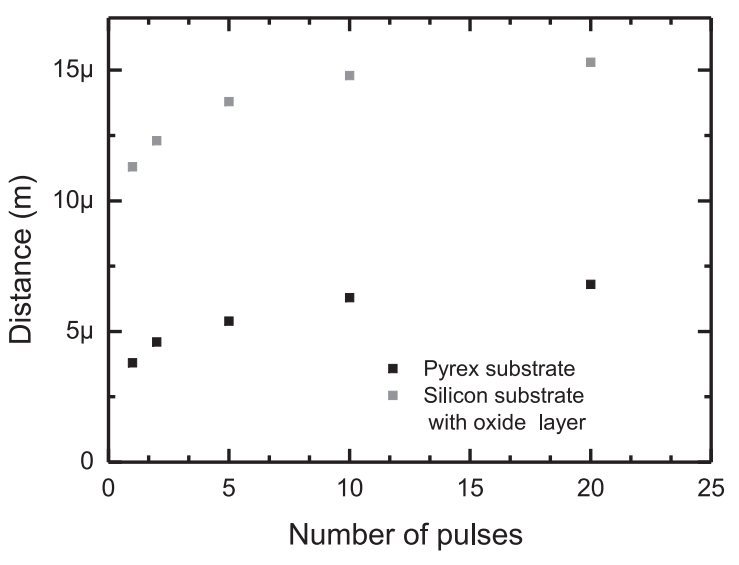

(b) Location

Figure 6.2: The distance from the heater (b) and temperature (a) of the point of maximum temperature in the liquid for a pyrex substrate and a silicon substrate with a $5 \mu \mathrm{m}$ silicon-oxide insulation layer. The temperature of the heater is also shown in (a) for both cases.

If a gas bubble is formed it will be situated at the point of maximum temperature. For a silicon substrate with an insulation layer, the gas bubble will be smaller or not present due to the lower maximum liquid temperature. Furthermore the gas bubbles will show less interference with the vapour bubble formation and growth, because they will be 
located at a larger distance from the heater. Therefore it is expected that the gas-bubble will start to have a negative effect on the performance of the bubble actuator at a higher repetition frequency. The use of a silicon substrate in combination with a silicon-oxide insulation layer will lead to an increase in the maximum repetition frequency.

\section{Increase in the heater surface area}

Not only the placement of the heaters is important, changes in the heater surface area might also lead to a better performance. The bubble volume is directly related to surface area. An increased heater surface will increase the generated bubble volume and is therefore expected to lead to a larger deflection. An increase in heater area will require more energy to generate a bubble and can also change the distribution of the current density in the heater. For large square heaters the maximum current density might be reached and this can lead to damage of the heater. The current density for these heaters will also be highly non-uniform. Here simulations of the current density can be used to improve the heater design and create a heater with a large surface area and highly uniform current density.

A vertical placement of the heaters on the substrate also increases the total surface area. It is expected that the bubble volume will increase and therefore the volume displacement is larger. More general any increase of the heater surface area will lead to a larger volume displacement, if a bigger bubble volume can be created.

\section{Parallel heaters}

It is also possible to use a number of heaters in parallel. By alternating the heating pulses between the different heaters the maximum repetition frequency is increased. The distance between the heaters should be chosen in such a way that they have no large thermal effect on each other. Multiple heaters in a single device could also have another advantage. It is known that the growing and collapse bubble will result in convection. The convection created by a bubble on one heater might lead to the displacement of a permanent bubble on another heater. This could result in the a faster collapse. The exact effect is however hard to predict, but some experiments on the interaction between bubbles created under high heat-flux conditions were done by Yang et al. [109].

\section{Ratio between heater and membrane surface area}

All the devices used in this research have a larger membrane area than heater area. For the $\mathrm{KOH}$ etched structures the opposite is also possible. The relatively large heater surface and converging structure of the cavity could lead to a considerable increase in deflection. Whether the maximum deflection actually will increase depends on the membrane size and the stiffness of the membrane. 


\subsubsection{Optimal properties of the liquid}

In this research only ethanol was used, but other liquids might be more suitable and could lead to an improvement in performance. Several different liquid properties will be important in the choice of the liquid. The liquid properties can be divided in four groups.

- Boiling temperature, Critical temperature and latent heat

- Solubility of gasses

- Thermal conductivity and diffusivity

- Viscosity and surface tension

\section{Boiling temperature, Critical temperature and latent heat}

The critical temperature, normal boiling temperature and latent heat of the liquid are important in the generation of bubbles. The pressure impulse is determined by the pressure inside the bubble right after bubble nucleation. This pressure is close to the vapour pressure at the nucleation temperature and can be calculated with equations (2.16) and (2.17) described in section 2.2.3. Liquids with a large latent heat and/or a large difference between the nucleation temperature at ambient pressure and spontaneous nucleation temperature will have a relatively large vapour pressure. An example of such a liquid is water. The pressure inside the bubble just after nucleation can be as high as 100 bar. It is therefore expected that the pressure impulse caused by bubble formation in water will be significantly higher than for ethanol and more useful work can be generated.

The bubble size will be closely related to the boiling temperature at pressure inside the cavity. At the bubble interface the conditions are nearly in thermal equilibrium. The bubble interface will be located close to the isotherm of the boiling temperature. The difference between the boiling temperature and ambient temperature determines the degree of subcooling. A large degree of subcooling will result in a large pressure difference between the vapour in the bubble and the liquid at the moment of maximum bubble volume. A large degree of subcooling will also speed-up the condensation process. A fast collapse of the vapour bubble will follow. Creating a bubble in these liquids will require more energy because the difference in temperature between the ambient and nucleation temperature will be larger. Therefore the efficiency will probably decrease with an increase in subcooling, the maximum repetition frequency, however, will increase.

\section{Solubility of gasses}

The maximum repetition frequency of the bubble actuator is limited by the formation of a permanent bubble. The influence of this bubble on the performance of the actuator depends on the on the amount of residual gas in the liquid. When there is no gas dissolved in the liquid the maximum repetition frequency will increase considerably. Degassing the liquid before use is therefore of extreme importance. However, it is nearly impossible to get rid of all the residual gas. The solubility of a gas in a liquid depends on the properties 
of the liquid as well as the gas. The solubility also depends on the temperature and pressure. In general the solubility increases with pressure.

The influence of the temperature is less straight forward; it can increase or decrease with temperature and depends on the liquid, the gas and the pressure. For the microbubble actuator a liquid with a high solubility for gasses is most preferable, because gas bubbles will quickly dissolve in the liquid. A liquid, for which the solubility increases with temperature in the temperature range reached in the liquid during actuation, would also highly beneficial, because this would restrict the formation of a gas bubble. If a gas bubble is formed in such a liquid, it will not be located at the point of maximum temperature but will move away from this position. The gas will not accumulate and the negative effect of gas bubbles on the performance of the actuator will be reduced. Unfortunately a high solubility makes degassing more difficult. A large amount of gas can be stored in the liquid, if the solubility is high. The amount of gas can be decreased by depressurizing the liquid before use. The equilibrium between gas in the liquid and gas in the air above the liquid will shift and less gas will be present in the liquid. If the pressure, however drops below the vapour pressure of the liquid, the liquid will also start to evaporate. A change in temperature can also improve the degassing. The solubility of the gasses expected to be present in the liquid, which in most cases is mostly air, ideally are at their minimum during degassing, but at their maximum at temperature around the heater during operation. Some trends and predictions of the solubility of gases in liquids were described by Leites [104] and can be used to find to the best liquid, a good degassing procedure and the optimal operating conditions for the explosive micro-bubble actuator.

\section{Thermal conductivity and diffusivity}

The thermal conductivity and diffusivity of the liquid determine the speed of temperature rise or decrease and the distribution of energy in the liquid. During the cooling period a high thermal conductivity and diffusivity will enable a fast dispersion of the energy and fast temperature decrease. For a liquid with a high conductivity and diffusivity, like water, also a smaller amount of the energy will be lost in the substrate. The fast distribution of the energy across the liquid will, however, also result in a slower temperature increase during the heating pulse. Therefore higher heat-fluxes or longer pulse times are required to generated bubbles. In total more energy will be used in the bubble formation, but the volume of liquid heated by the pulse will also be larger. This larger heated volume will probably lead to a larger bubble. Experiments or more accurate simulations have to determine, whether or not the efficiency increases or a higher maximum repetition frequency can be attained for liquids with a higher conductivity or diffusivity.

\section{Viscosity and surface tension}

The viscosity and surface tension of the liquid have an influence on the dynamics of the bubble and the liquid surrounding it. A relatively low viscosity will lead to larger bubbles. A decrease in surface tension will have an identical effect. The influence of viscosity and surface tension on the hydrodynamics are even more important. The hydrodynamics determine the heat-transfer by convection and will influence the bubble collapse. This will have an effect on the cooling of the liquid after the heating pulse and dissolution of 
the gas into the liquid. However due to the complexity of the bubble dynamics and heattransfer during the bubble collapse, it is difficult to predict the influence of the viscosity and surface tension.

Using a different working liquid will result in large number of changes in the system. In order to generate more force a liquid with a large latent heat and large difference between the boiling temperature and critical temperature can be used. Off course the heater should be able to heat the liquid to temperature close to the critical temperature. For the heaters in the micro-bubble actuator the maximum temperature will be around $600^{\circ} \mathrm{C}$ and there for the critical temperature of the liquid should be lower than $670^{\circ} \mathrm{C}$. The maximum frequency can be increased by using liquids with a high conductivity or diffusivity. A liquid with a high diffusivity will generate a larger bubble volume and therefore larger deflections. The maximum repetition frequency can also be increased by using a liquid with a higher solubility for the most common gasses or a better degassing procedure.

\subsubsection{Membrane}

The size, geometry and properties of the membrane will have an influence on the initial deflection and vibrations following afterwards. The ideal response to a heating pulse is a large and single deflection peak. After this deflection the membrane should quickly return to its initial state without large vibrations. Several improvements to the membrane could be made to get closer to the ideal response. A straightforward way modify the response is to change the thickness of the membrane. A change in thickness of the membrane alters the flexibility of the membrane. An increase in stiffness decreases the amplitude of vibrations and increase their natural frequencies. The ratio between the pressure impulse generated by the bubbles and the force needed for membrane deflection will determine if this will also result in a decrease in maximum deflection. A decrease of the surface area will have a similar effect. Furthermore a change in ratio between heater area and membrane area might result in an increase in deflection and a more favourable distribution of the excited mode-shapes. The use of another membrane material, like an elastomer, can lead to much larger change in response, but a full investigation of the effect of use of these materials on the response is beyond the scope of this thesis. The same holds for the effect of several more complicated membrane structures. Here one can think of the use a corrugated membrane, a membrane with a boss, a bi-stable membrane or a membrane with reinforcing beams to make it less flexible in certain directions.

\subsection{Applications}

The explosive micro-bubble can create a deflection of several microns and displace a volume of $3.0 \times 10^{-13} \mathrm{~m}^{3}$ at frequencies of several $\mathrm{kHz}$. It is not possible for this actuator to provide a continuous deflection; it can only give pulses of a few microseconds.

One of the most promising applications pump is therefore a small pump. In a large number of systems pumps are used to transport liquid or gas. As these systems get smaller, smaller pumps are required. The smaller systems often also operate at higher 
frequencies and therefore the actuator has to be fast. A single micro-bubble actuator placed in a small channel with a nozzle in the outlet and a diffuser in the inlet of the channel. The bubble actuator creates a volume change in the channel and due to the nozzle and diffuser a net flow from the inlet to the outlet will be created. By placing a number of bubble actuators along the length of a channel a pump can also be created. If the actuation is periodically cycled a net-flow is generated. The advantage of this type of pump is that fluid can be transported in both directions. The disadvantage of this type of pump is that in order create a liquid flow the timing of the displacement of each individual membrane has to be perfect.

The micro-bubble actuator can be placed in a reservoir with nozzle on another side of the reservoir; like in a inkjet-printerhead. The pressure created by the formation of a bubble will result in the ejection of a droplet of liquid. In the arrangement proposed here the liquid in which the bubble is created is different from the liquid that will be ejected though the nozzle. This separation enables the printing of a wide variety of liquids. If the system is designed correctly the working liquid and the liquid that has to be ejected can be separated thermally. Liquids, which are temperature sensitive like water containing DNA, can be printed without being heated. It is also possible to heat the printing liquid without changing the bubble generation and thus the actuator performance. This will enable the printing of even more liquids.

The actuator can be used produce sound and/or pressure waves. The performance of the cryocooler designed by Vanapalli et al [110] could be improved if smaller actuators are used. The bubble actuator is considerable smaller than the piezoelectric actuator used in their research and could therefore lead to an improvement of the performance. The actuator can also be used as a loudspeaker to produce sound in the kilohertz range. If a large number of these actuators are placed in parallel it is possible to do experiments on non-linear acoustics.

Although the bubble actuator can not generate a permanent deflection it can be used as a valve. The most simple valve will be a normally opened valve. The displacement of the membrane can be used to temporarily close a channel or to regulate the total flow through a channel. Creating a normally closed valve is also possible, but this would require a more complex design. Furthermore the displacement of the membrane can be used to move another structure, for example a small mirror in a photonic system.

The explosive micro-bubble actuator has several advantages above piezoelectric actuators with a comparable performance. The micro-bubble actuator is relatively easy to fabricate with standard technology, while the fabrication of a piezo-actuator is made from less standard materials and often requires special and complex layer growth techniques to create the piezoelectric material. Furthermore in most cases these material can not be machined by standard MEMS technology. The bubble actuator can be fabricated on a wafer scale and can easily combined with other devices, like sensors or fluidic devices made with MEMS technology. Integration in other systems for example made from PDMS, which is a material used often for micro-fluidic devices, can also be achieved relatively easy. Another advantage of the bubble actuator in comparison to the piezo actuator is the low voltage that is required to generate the bubbles. Piezoelectric devices need several hundreds of Volts to generate a force, where the bubble-actuator can be designed to use only a few volts. The electronics to control the actuation and provide the power used in the bubble 
generation can therefore be relatively simple.

The design of the actuator discussed in this thesis was not optimized for a specific application, neither was it perfected to reach a certain maximum performance. If the application of the actuator is known the design can be optimized to improve the performance for that particular application. In a pump the displaced volume is the most important parameter and the actuator can be improved to develop a larger volume change. For other applications the force generated at the centre of the membrane or the repetition frequency are more important. A small change in the membrane design or pressure inside the cavity can already be enough to enhance the performance significantly. The design improvements discussed in the previous section can also be used to meet the requirements of a specific application. 


\section{References}

[1] X. Yang, C. Grosjean, Y.C. Tai, and C.M. Ho. A mems thermopneumatic silicone rubber membrane valve. Sensors and Actuators A-Physical, 64(1):101-108, January 1998.

[2] X. Yang, C. Grosjean, and Y.C. Tai. Design, fabrication, and testing of micromachined silicone rubber membrane valves. Journal of Microelectromechanical Systems, 8(4):393402, December 1999.

[3] C. Goll, W. Bacher, B. Bustgens, D. Maas, W. Menz, and W.K. Schomburg. Microvalves with bistable buckled polymer diaphragms. Journal of Micromechanics and Microengineering, 6(1):77-79, March 1996.

[4] O.C. Jeong and S.S. Yang. Fabrication and test of a thermopneumatic micropump with a corrugated p plus diaphragm. Sensors and Actuators A-Physical, 83(1-3):249-255, May 2000 .

[5] W.I. Jang, C.A. Choi, C.H. Jun, Y.T. Kim, and M. Esashi. Surface micromachined thermally driven micropump. Sensors and Actuators A-Physical, 115(1):151-158, September 2004 .

[6] J.H. Kim, K.H. Na, C.J. Kang, D. Jeon, and Y.S. Kim. A disposable thermopneumaticactuated microvalve stacked with pdms layers and ito-coated glass. Microelectronic Engineering, 73-74:864-869, June 2004.

[7] M. Knight and J. House. Design, fabrication, and test of a peristaltic micropump. Microsystem Technologies-Micro-and Nanosystems-Information Storage and Processing Systems, 10(5):426-431, August 2004.

[8] A. Wego and L. Pagel. A self-filling micropump based on pcb technology. Sensors and Actuators A-Physical, 88(3):220-226, March 2001.

[9] A. Wego, H.W. Glock, L. Pagel, and S. Richter. Investigations on thermo-pneumatic volume actuators based on pcb technology. Sensors and Actuators A-Physical, 93(2):95102, September 2001.

[10] H. Takao, K. Miyamura, H. Ebi, M. Ashiki, K. Sawada, and M. Ishida. A mems microvalve with pdms diaphragm and two-chamber configuration of thermo-pneumatic actuator for integrated blood test system on silicon. Sensors and Actuators A-Physical, 119(2):468475, April 2005. 
[11] F.C.M. Van de Pol, D.G.J. Wonnink, M. Elwenspoek, and J.H.J. Fluitman. A thermopneumatic actuation principle for a microminiature pump and other micromechanical devices. Sensors and Actuators, 17(1-2):139-143, May 1989.

[12] F.C.M. Van de Pol, H.T.G. Van Lintel, M. Elwenspoek, and J.H.J. Fluitman. A thermopneumatic micropump based on micro-engineering techniques. Sensors and Actuators A: Physical, 21(1-3):198-202, February 1990.

[13] C.A. Rich and K.D. Wise. A high-flow thermopneumatic microvalve with improved efficiency and integrated state sensing. Journal of Microelectromechanical Systems, 12(2):201-208, April 2003.

[14] W.Y. Sim, H.J. Yoon, O.C. Jeong, and S.S. Yang. A phase-change type micropump with aluminum flap valves. Journal of Micromechanics and Microengineering, 13(2):286-294, March 2003.

[15] P.L. Bergstrom, J. Ji, Y.N. Liu, M. Kaviany, and K.D. Wise. Thermally driven phasechange microactuation. Journal of Microelectromechanical Systems, 4(1):10-17, March 1995.

[16] E.H. Yang, C. Lee, J. Mueller, and T. George. Leak-tight piezoelectric microvalve for highpressure gas micropropulsion. Journal of Microelectromechanical Systems, 13(5):799-807, October 2004.

[17] E.T. Carlen and C.H. Mastrangelo. Surface micromachined paraffin-actuated microvalve. Journal of Microelectromechanical Systems, 11(5):408-420, October 2002.

[18] J.C. Yoo, Y.J. Choi, C.J. Kang, and Y.S. Kim. A novel polydimethylsiloxane microfluidic system including thermopneumatic-actuated micropump and paraffin-actuated microvalve. Sensors and Actuators A-Physical, 139(1-2):216-220, September 2007.

[19] A.K. Henning, J.S. Fitch, J.M. Harris, E.B. Dehan, B.A. Cozad, L. Christel, Y. Fathi, D.A. Hopkins, L.J. Lilly, W. McCulley, W.A. Weber, and M. Zdeblick. Microfluidic mems for semiconductor processing. Ieee Transactions on Components Packaging and Manufacturing Technology Part B-Advanced Packaging, 21(4):329-337, November 1998.

[20] O.C. Jeong, S.W. Park, S.S. Yang, and J.J. Pak. Fabrication of a peristaltic pdms micropump. Sensors and Actuators A-Physical, 123-24:453-458, September 2005.

[21] J. Fahrenberg, W. Bier, D. Maas, W. Menz, R. Ruprecht, and W.K. Schomburg. A microvalve system fabricated by thermoplastic molding. Journal of Micromechanics and Microengineering, 5(2):169-171, June 1995.

[22] A. Asai, T Hara, and I Endo. One-dimensional model of bubble growth and liquid flow in bubble jet printers. Japanese Journal of Applied Physics, 26(10):1794-1801, July 1987.

[23] P.H. Chen, W.C. Chen, and S.H. Chang. Bubble growth and ink ejection process of a thermal ink jet printhead. International Journal of Mechanical Sciences, 39(6):683-\&, June 1997. 
[24] F.G. Tseng, C.J. Kim, and C.M. Ho. A high-resolution high-frequency monolithic topshooting microinjector free of satellite drops - part i: Concept, design, and model. Journal of Microelectromechanical Systems, 11(5):427-436, October 2002.

[25] F.G. Tseng, C.J. Kim, and C.M. Ho. A high-resolution high-frequency monolithic topshooting microinjector free of satellite drops - part ii: Fabrication, implementation, and characterization. Journal of Microelectromechanical Systems, 11(5):437-447, October 2002 .

[26] T.G. Kang and Y.H. Cho. A four-bit digital microinjector using microheater array for adjusting the ejected droplet volume. Journal of Microelectromechanical Systems, 14(5):1031-1038, October 2005.

[27] T.G. Kang, S.W. Kim, and Y.H. Cho. High-impulse, low-power, digital microthrusters using low boiling temperature liquid propellant with high viscosity fluid plug. Sensors and Actuators A-Physical, 97-8:659-664, April 2002.

[28] J.H. Tsai and L.W. Lin. A thermal-bubble-actuated micronozzle-diffuser pump. Journal of Microelectromechanical Systems, 11(6):665-671, December 2002.

[29] J.Y. Jung and H.Y. Kwak. Fabrication and testing of bubble powered micropumps using embedded microheater. Microfluidics and Nanofluidics, 3(2):161-169, April 2007.

[30] Z.Z. Yin and A. Prosperetti. A microfluidic 'blinking bubble' pump. Journal of Micromechanics and Microengineering, 15(3):643-651, March 2005.

[31] T.K. Jun and C.J. Kim. Valveless pumping using traversing vapor bubbles in microchannels. Journal of Applied Physics, 83(11):5658-5664, June 1998.

[32] H. Yuan and A. Prosperetti. The pumping effect of growing and collapsing bubbles in a tube. Journal of Micromechanics and Microengineering, 9(4):402-413, December 1999.

[33] W. van der Wijngaart, D. Chugh, E. Man, J. Melin, and G. Stemme. A low-temperature thermopneumatic actuation principle for gas bubble microvalves. Journal of Microelectromechanical Systems, 16(3):765-774, June 2007.

[34] L. Lin, A.P. Pisano, and A.P. Lee. Microbubble powered actuator. Proceedings of the International Conference on Solid-State Sensors and Actuators, pages 1041-1044, 1991.

[35] H.J. Lee, Y.S. Chang, Y.P. Lee, K.H. Jeong, and H.Y. Kim. Deflection of microcantilever by growing vapor bubble. Sensors and Actuators A-Physical, 136(2):717-722, May 2007.

[36] A.P. Papavasiliou, D. Liepmann, and A.P. Pisano. Fabrication of a free floating silicon gate valve. In Proceedings of the ASME MEMS Division, IMECE, volume 1, pages 435-440, 1999.

[37] D.S. Meng, Y. Ju, and C.J. Kim. A comparative study of electrolysis and boiling for bubble-driven microactuations. volume 2, pages 1263-1266, 2005.

[38] C.R. Neagu, J.G.E. Gardeniers, M. Elwenspoek, and J.J. Kelly. An electrochemical active valve. Electrochimica Acta, 42(20-22):3367-3373, 1997. 
[39] A.P. Papavasiliou, D. Liepmann, and A.P. Pisano. Electrolysis-bubble actuated gate valve. In Proceedings 2000 Solid State Sensor and Actuator Workshop, pages 48-51, 2000.

[40] C.T. Ho, R.Z. Lin, H.Y. Chang, and C.H. Liu. Micromachined electrochemical t-switches for cell sorting applications. Lab on A Chip, 5(11):1248-1258, 2005.

[41] M.S. Plesset and S.A Zwick. The growth of vapor bubbles in superheated liquids. Journal of Applied Physics, 25:493-501, 1954.

[42] M. Blander and L.J. Katz. Bubble nucleation in liquids. American Institute of Chemical Engineers Journal, 21(5):833-848, 1979.

[43] V.P. Skripov. Metastable Liquids. John Wiley \& Sons, Inc., New York, 1972.

[44] S van Stralen and R Cole. Boiling Phenomena. Number 1. Hemisphere Publishing Corporation, 1979.

[45] C.F. Delale, J. Hruby, and F. Marsik. Homogeneous bubble nucleation in liquids: The classical theory revisited. Journal of Chemical Physics, 118(2):792-806, January 2003.

[46] A.A. Avdeev and Y.B. Zudin. Inertial-thermal governed vapor bubble growth in highly superheated liquid. Heat and Mass Transfer, 41(10):855-863, August 2005.

[47] S. Heo, S. Koshizuka, and Y. Oka. Numerical analysis of boiling on high heat-flux and high subcooling condition using mps-mafl. International Journal of Heat and Mass Transfer, 45(13):2633-2642, June 2002.

[48] S.A.D. Wiesche, C. Rembe, and E.P. Hofer. Boiling of superheated liquids near the spinodal: I general theory. Heat and Mass Transfer, 35(1):25-31, May 1999.

[49] J. Li, G.P. Peterson, and P. Cheng. Mechanical nonequilibrium considerations in homogeneous bubble nucleation for unsteady-state boiling. International Journal of Heat and Mass Transfer, 48(15):3081-3096, July 2005.

[50] Yu Varlamov, Yu Meshcheryakov, S.I. Lezhnin, M.R. Predtechenskii, and S.N. Ul'yankin. Evolution of a vapor cavity during explosive boiling on a film microheater: Experiment and numerical simulation. Journal of Applied Mechanics and Technical Physics, 48(4):534-541, 2007.

[51] J.L. Xu and W. Zhang. Effect of pulse heating parameters on the microscale bubble dynamics at a microheater surface. International Journal of Heat and Mass Transfer, 51(1-2):389-396, January 2008.

[52] K. Okuyama and Y. Iida. Transient boiling heat-transfer characteristics of nitrogen (bubble behavior and heat-transfer rate at stepwise heat-generation). International Journal of Heat and Mass Transfer, 33(10):2065-2071, October 1990.

[53] K.P. Derewnicki. Experimental studies of heat-transfer and vapor formation in fast transient boiling. International Journal of Heat and Mass Transfer, 28(11):2085-2092, 1985.

[54] K. Okuyama, Y. Kozawa, A. Inoue, and S. Aoki. Transient boiling heat-transfer characteristics of r113 at large stepwise power-generation. International Journal of Heat and Mass Transfer, 31(10):2161-2174, October 1988. 
[55] A. Asai. Application of the nucleation theory to the design of bubble jet printers. Japanese Journal of Applied Physics Part 1-Regular Papers Short Notes 83 Review Papers, 28(5):909-915, May 1989.

[56] A. Asai. Bubble dynamics in boiling under high heat-flux pulse heating. Journal of Heat Transfer-Transactions of the Asme, 113(4):973-979, November 1991.

[57] A. Asai. 3-dimensional calculation of bubble-growth and drop ejection in a bubble jet printer. Journal of Fluids Engineering-Transactions of the Asme, 114(4):638-641, December 1992.

[58] Y. Iida, K. Okuyama, and K. Sakurai. Peculiar bubble generation on a film heater submerged in ethyl-alcohol and imposed a high heating rate over $10(7) \mathrm{k} \mathrm{s}(-1)$. International Journal of Heat and Mass Transfer, 36(10):2699-2701, July 1993.

[59] Y. Iida, K. Okuyama, and K. Sakurai. Boiling nucleation on a very small film heater subjected to extremely rapid heating. International Journal of Heat and Mass Transfer, 37(17):2771-2780, November 1994.

[60] C.T. Avedisian, W.S. Osborne, F.D. Mcleod, and C.M. Curley. Measuring bubble nucleation temperature on the surface of a rapidly heated thermal ink-jet heater immersed in a pool of water. Proceedings of the Royal Society of London Series A-Mathematical Physical and Engineering Sciences, 455(1991):3875-3899, November 1999.

[61] L.W. Lin, A.P. Pisano, and V.P. Carey. Thermal bubble formation on polysilicon micro resistors. Journal of Heat Transfer-Transactions of the Asme, 120(3):735-742, August 1998.

[62] K. Okuyama, S. Tsukahara, N. Morita, and Y. Iida. Transient behavior of boiling bubbles generated on the small heater of a thermal ink jet printhead. Experimental Thermal and Fluid Science, 28(8):825-834, October 2004.

[63] P.G. Deng, Y.K. Lee, and P. Cheng. The growth and collapse of a micro-bubble under pulse heating. International Journal of Heat and Mass Transfer, 46(21):4041-4050, October 2003.

[64] P.G. Deng, Y.K. Lee, and P. Cheng. An experimental study of heater size effect on micro bubble generation. International Journal of Heat and Mass Transfer, 49(15-16):25352544, July 2006.

[65] Y. Hong, N. Ashgriz, and J. Andrews. Experimental study of bubble dynamics on a micro heater induced by pulse heating. Journal of Heat Transfer-Transactions of the Asme, 126(2):259-271, April 2004.

[66] J.Y. Jung, J.Y. Lee, H.C. Park, and H.Y. Kwak. Bubble nucleation on micro line heaters under steady or finite pulse of voltage input. International Journal of Heat and Mass Transfer, 46(20):3897-3907, September 2003.

[67] L.W. Lin. Thermal challenges in mems applications: phase change phenomena and thermal bonding processes. Microelectronics Journal, 34(3):179-185, March 2003. 
[68] Z. Yin, A. Prosperetti, and J. Kim. Bubble growth on an impulsively powered microheater. International Journal of Heat and Mass Transfer, 47(5):1053-1067, February 2004.

[69] C.T. Avedisian, R.E. Cavicchi, and M.J. Tarlov. New technique for visualizing microboiling phenomena and its application to water pulse heated by a thin metal film. Review of Scientific Instruments, 77(6):-, June 2006.

[70] Y.S. Hong, N. Ashgriz, J. Andrews, and H. Parizi. Numerical simulation of growth and collapse of a bubble induced by a pulsed microheater. Journal of Microelectromechanical Systems, 13(5):857-869, October 2004.

[71] K. Okuyama, S. Mori, K. Sawa, and Y. Iida. Dynamics of boiling succeeding spontaneous nucleation on a rapidly heated small surface. International Journal of Heat and Mass Transfer, 49(15-16):2771-2780, July 2006.

[72] Yu Varlamov, Yu Meshcheryakov, M.P. Predtechenskii, S.I. Lezhnin, and S.N. Ul'yankin. Specific features of explosive boiling of liquids on a film microheater. Journal of Applied Mechanics and Technical Physics, 48(2):213-220, 2007.

[73] Z. Zhao, S. Glod, and D. Poulikakos. Pressure and power generation during explosive vaporization on a thin-film microheater. International Journal of Heat and Mass Transfer, 43(2):281-296, January 2000.

[74] S. Glod, D. Poulikakos, Z. Zhao, and G. Yadigaroglu. An investigation of microscale explosive vaporization of water on an ultrathin pt wire. International Journal of Heat and Mass Transfer, 45(2):367-379, January 2002.

[75] P.G. Deng, Y.K. Lee, and P. Cheng. Micro bubble dynamics in dna solutions. Journal of Micromechanics and Microengineering, 14(5):693-701, May 2004.

[76] P.G. Deng, Y.K. Lee, and P. Cheng. Measurements of micro bubble nucleation temperatures in dna solutions. Journal of Micromechanics and Microengineering, 15(3):564-574, March 2005.

[77] P.G. Deng, Y.K. Lee, and P. Cheng. Two-dimensional micro-bubble actuator array to enhance the efficiency of molecular beacon based dna micro-biosensors. Biosensors 86 Bioelectronics, 21(8):1443-1450, February 2006.

[78] K. Okuyama, J.H. Kim, and Y. Iida. Allowable repetition frequency of pulse heating in microactuators using rapid boiling (effects of heater size and substrate material). Jsme International Journal Series B-Fluids and Thermal Engineering, 46(3):399-407, August 2003.

[79] A. Bejan. Heat transfer. John Wiley\&Sons, New York, 1993.

[80] Q.H. Tang and J.L. Xu. A three-dimensional analytical solution of the microheater temperature before bubble nucleation. Applied Thermal Engineering, 28(11-12):1491-1501, August 2008.

[81] B.B. Mikic, W.M. Rohsenow, and P. Griffith. On bubble growth rates. International Journal of Heat and Mass Transfer, 13(4):657-\&, 1970. 
[82] Lord Rayleigh. On the pressure developed in a liquid during the collapse of a spherical cavity. Philosophical Magazine, 34:94-98, 1917.

[83] M Di Giovanni. Flat and Corrugated Diaphragm Design Handbook. Marcel Dekker Inc., New York, 1982.

[84] A.B. Wood. A Textbook of Sound. G. Bell and Sons Ltd, London, 1964.

[85] S Timoshenko and W Woinowsky-Krieger. Theory of Plates and Shells. McGraw-Hill, 1959.

[86] S Timoshenko, D.H. Young, and W Weaver JR. Vibration Problems in Engineering. John Wiley \& sons, Inc, 1974.

[87] H. Lamb. On the vibrations of an elastical plate in contact with water. Proceedings of the Royal Society of London.Series A, Containing Papers of Mathematical and Physical Character., 98(690):205-216, March 1920.

[88] M.K. Kwak. Vibration of circular plates in contact with water. Journal of Applied Mechanics-Transactions of the Asme, 58(2):480-483, June 1991.

[89] M.K. Kwak. Vibration of circular membranes in contact with water. Journal of Sound and Vibration, 178(5):688-690, December 1994.

[90] G. Romera-Guereca, J. Lichtenberg, A. Hierlemann, D. Poulikakos, and B. Kang. Explosive vaporization in microenclosures. Experimental Thermal and Fluid Science, 30(8):829836, August 2006.

[91] K.M. Balss, C.T. Avedisian, R.E. Cavicchi, and M.J. Tarlov. Nanosecond imaging of microboiling behavior on pulsed-heated au films modified with hydrophilic and hydrophobic self-assembled monolayers. Langmuir, 21(23):10459-10467, November 2005.

[92] P. Selvaganapathy, E.T. Carlen, and C.H. Mastrangelo. Electrothermally actuated inline microfluidic valve. Sensors and Actuators A-Physical, 104(3):275-282, May 2003.

[93] C.M. Cheng and C.H. Liu. A capillary system with thermal-bubble-actuated 1 x n microfluidic switches via time-sequence power control for continuous liquid handling. Journal of Microelectromechanical Systems, 15(2):296-307, April 2006.

[94] P. Gluche, R. Leuner, A. Vescan, W. Ebert, E. Kohn, C. Rembe, S. der Wiesche, and E.P. Hofer. Actuator sensor technology on "electronic grade" diamond films. Microsystem Technologies, 5(1):38-43, October 1998.

[95] T. McGee. Principles and methods of Temperature measurement. Wiley, New York, 1988.

[96] R.M. Tiggelaar, P.W.H. Loeters, P. van Male, R.E. Oosterbroek, J.G.E. Gardeniers, M.H.J.M. de Croon, J.C. Schouten, M.C. Elwenspoek, and A. van den Berg. Thermal and mechanical analysis of a microreactor for high temperature catalytic gas phase reactions. Sensors and Actuators A-Physical, 112(2-3):267-277, May 2004.

[97] B.H. Jo and R.W. Vook. In-situ ultra-high-vacuum studies of electromigration in copperfilms. Thin Solid Films, 262(1-2):129-134, June 1995. 
[98] P. Enoksson. New structure for corner compensation in anisotropic koh etching. Journal of Micromechanics and Microengineering, 7(3):141-144, September 1997.

[99] B. Kim and D.I.D. Cho. Aqueous koh etching of silicon (110) - etch characteristics and compensation methods for convex corners. Journal of the Electrochemical Society, 145(7):2499-2508, July 1998.

[100] B. Wacogne, Z. Sadani, and T. Gharbi. Compensation structures for v-grooves connected to square apertures in koh-etched (100) silicon: theory, simulation and experimentation. Sensors and Actuators A-Physical, 112(2-3):328-339, May 2004.

[101] G. Romera-Guereca, T.Y. Choi, and D. Poulikakos. Explosive vaporization and microbubble oscillations on submicron width thin film strip heaters. International Journal of Heat and Mass Transfer, 51(17-18):4427-4438, August 2008.

[102] B.C. Khoo, E. Klaseboer, and K.C. Hung. A collapsing bubble-induced micro-pump using the jetting effect. Sensors and Actuators A-Physical, 118(1):152-161, January 2005.

[103] P.S. Epstein and M.S. Plesset. On the stability of gas bubbles in liquid-gas solutions. Journal of Chemical Physics, 18(11):1505-1509, 1950.

[104] I.L. Leites. Some trends and a prediction of the solubility of gases in liquids and the heat of dissolution. Separation and Purification Technology, 12(3):201-213, December 1997.

[105] J.G.E. Gardeniers, H.A.C. Tilmans, and C.C.G. Visser. Lpcvd silicon-rich silicon nitride films for applications in micromechanics, studied with statistical experimental design. Journal of Vacuum Science \& Technology A-Vacuum Surfaces and Films, 14(5):28792892, September 1996.

[106] L. Kiesewetter, J.M. Zhang, D. Houdeau, and A. Steckenborn. Determination of young moduli of micromechanical thin-films using the resonance method. Sensors and Actuators A-Physical, 35(2):153-159, December 1992.

[107] V.L. Spiering, S. Bouwstra, J.F. Burger, and M. Elwenspoek. Membranes fabricated with a deep single corrugation for package stress reduction and residual stress relief. Journal of Micromechanics and Microengineering, 3(4):243-246, 1993.

[108] J.H. Powell and J.H.T. Roberts. On the frequency of vibration of circular diaphragms. Proceedings of the Physical Society of London, 35(1):170-182, 1922.

[109] I.D. Yang, F.G. Tseng, R.J. Yu, and C.C. Chieng. Bubble dynamics for explosive microthermal dual bubbles. Journal of Microelectromechanical Systems, 16(3):734-745, June 2007.

[110] S. Vanapalli, H.J.M. ter Brake, H.V. Jansen, Y. Zhao, H.J. Holland, J.F. Burger, and M.C. Elwenspoek. High frequency pressure oscillator for microcryocoolers. Review of Scientific Instruments, 79(4):-, April 2008. 


\section{Summary}

Microactuators are key components in numerous microsystems, and in many applications strong and fast microactuators are required. The principles used to generate forces in the current actuators are not capable of fulfilling both requirements at the same time, so new principles have to be investigated. One promising technique is based on explosive evaporation. Explosive evaporation occurs when a liquid is exposed to extremely high heat-fluxes. Within a few microseconds the liquid reaches a temperature close to the critical point. At these temperatures spontaneous nucleation can take place; the nucleation barrier is lowered significantly and small fluctuations in the liquid density will act as bubble nuclei. A large number of small bubbles will be formed at numerous sites. Nucleated bubbles instantly coalesce forming a vapour film followed by rapid expansion due to the pressure impulse. This effect of explosive evaporation, which already has proven its use in current thermal inkjet technology, can be used to produce mechanical work. Here the force generated by bubble formation and growth will be utilized to deflect a flexible membrane.

Within this thesis the design and fabrication of the explosive micro-bubble actuator is described. The influence of different operating conditions and design parameters on the performance of the actuator is determined. The behaviour of the actuator is highly dependent on the generation and dynamics of the created bubbles. The study of the bubbles created due to explosive evaporation increases the insight in the complex dynamics and provides experimental data to verify the validity of newly developed models. This information is used to find the best operating conditions and create design rules for future devices.

The actuator is fabricated using MEMS technology. A pyrex substrate with thin film heaters of platinum form the bottom of a cavity etched in silicon. The top of the cavity is sealed by a flexible silicon-nitride membrane. This cavity is filled with degassed ethanol. Inside the actuator, bubbles are created by applying potential difference across a micrometer sized heater for a few microseconds. The power generated by these current pulses is dissipated inside and around the heater resulting in extremely high heat-fluxes up to several hundreds of $\mathrm{MW} / \mathrm{m}^{2}$ and temperature rise rates of $10^{8} \mathrm{~K} / \mathrm{s}$. These conditions result in spontaneous nucleation. A high degree of superheat is concentrated in a small area. The fact that only a small volume is heated leads to short cooling times and fast bubble collapse. This microsecond heating time, together with a high reproducibility makes the device extremely fast for a thermo-pneumatic actuator.

Information on the bubble generation and the bubble dynamics inside the bubble actuator was gained from images taken by stroboscopic imaging and by monitoring the heater temperature during the heating pulse with resistance thermometry. The influence 
of different operating and design parameters, such as heating power, pulse length and heater geometry on the moment of nucleation, the nucleation temperature, the bubble growth speed and the bubble size was investigated. In contrast to the research on the thermal bubble jet printers and most other studies on bubble generation under high heatflux conditions, the bubbles in the explosive micro-bubble actuator are generated in a closed system. Therefore the influence of the pressure inside the cavity on the bubble generation and dynamics were also examined. The experimental results are used to verify the validity of models on the heat-transfer in system and the effect of a non-uniform current density though the heater.

A bubble formed in this closed system by a short heating pulse will result in a dynamic deflection of the membrane. This dynamic membrane displacement is measured in realtime with a scanning laservibrometer. The membrane deflection is not only affected by the dynamics of the bubble, but also depends on the design of the actuator, the material properties of the membrane and the pressure inside the cavity. A constant pressure difference across the membrane will result in a permanent membrane deflection, which can be measured with a white light interferrometer. Measurements on this constant deflection were used to calibrate the membrane. After a bubble collapses the membrane is left in free-vibration and the properties of the membrane and the liquid inside the cavity will determine the movement of the membrane.

The maximum repetition frequency is reached when the previous bubble has just vanished at the moment a new bubble is created. At higher frequencies a permanent bubble will form and the performance of the actuator will decrease drastically. Although the ethanol is degassed before use, a small amount of residual gas is still present. A bubble created by explosive evaporation will therefore not only contain vapour, but also some gas. This gas has to dissolve into the liquid and this process is much slower than condensation. The amount of gas inside the bubble determines the bubble collapse time. An effective way to increase the maximum repetition frequency is to increase the pressure inside the cavity. This will increase solubility of the gas in the liquid and will change the dynamics of the bubble; the time it will take a bubble to collapse decreases. Although a small decrease in bubble size and membrane deflection will also occur, it will lead to a considerable increase in maximum repetition frequency.

The best performance achieved in this research is a $2 \mu \mathrm{m}$ dynamic deflection for a repetition frequency of $5 \mathrm{kHz}$ at a cavity pressure of nearly $3 \mathrm{bar}$. At low repetition frequencies and ambient pressure even a higher membrane deflection of $6 \mu \mathrm{m}$ and a membrane displacement speed of $1.3 \mathrm{~m} / \mathrm{s}$ were measured. The total energy required to generate a bubble is several micro Joules. Only a fraction of this thermal energy is converted to mechanical energy, but due to the high energy density, the actuator is still powerful. The pressure inside the initial bubble is estimated at 25 bar and the growth speed of a bubble is several tens of $\mathrm{m} / \mathrm{s}$. The local heating also enables a fast bubble collapse. A reduction of the amount of residual gas, for example by an improved degassing procedure, could increase the maximum frequency to more than $50 \mathrm{kHz}$. The explosive micro-bubble actuator proves to be fast and can create a considerable deflection. It is not possible for this actuator to provide a continuous deflection; it can only give pulses. Therefore on can think about applications such as fast micro-pumps, loudspeakers, micro-compressors and inchworm actuators. 


\section{Samenvatting}

Microactuatoren zijn sleutel componenten in verscheidene microsystemen, en in veel toepassingen zijn krachtige and snelle actuatoren gewenst. De methodes die in de huidige actuatoren worden gebruikt om een kracht te genereren zijn niet in staat om aan beide eisen te voldoen, daarom moeten nieuwe methodes worden onderzocht. Een veelbelovende techniek is gebaseerd op explosieve verdamping. Explosieve verdamping kan plaatsvinden, wanneer een vloeistof wordt blootgesteld aan een extreem hoge warmtestroom. Binnen enkele microseconden bereikt de vloeistof een temperatuur dichtbij het kritische punt. Bij deze temperaturen kan spontane nucleatie plaats vinden, dat zich kenmerkt door de vorming van een groot aantal belletjes in de vloeistof. Deze belletjes zullen zich vrijwel onmiddellijk samenvoegen tot een damplaag, waarbij de gegenereerde druk impuls zal zorgen voor een snelle expansie. Deze explosieve verdamping heeft zich al bewezen in de huidige thermische inkjet technologie en kan worden gebruikt om mechanische arbeid te verrichten. In dit onderzoek zal de kracht die wordt gegenereerd bij het vormen en groeien van een bel, worden gebruikt om een flexibel membraan te laten bewegen.

In dit proefschrift worden het ontwerp en de fabricage van de exposieve micro-bellen actuator behandeld. De invloed van verschillende operationele parameters en ontwerpvariabelen op de prestatie van de actuator is onderzocht. Het gedrag van de actuator wordt voornamelijk bepaald door formatie en de dynamica van de bellen. De studie van de bellen, gevormd door explosieve verdamping, heeft het inzicht in de complexe dynamica vergroot en de experimenten hebben de data verschaft die gebruikt wordt om de geldigheid van nieuwe modellen te verifiëren. Deze informatie is vervolgens gebruikt om de beste operationele parameters te bepalen en nieuwe ontwerpregels voor toekomstige actuatoren te maken.

De actuator is vervaardigd met behulp van MEMS technologie. Een Pyrex substraat met een platina verwarmingselement van een aantal micrometers vormt de bodem van een in silicium geëtste holte. De bovenkant van de holte wordt afgesloten door een flexibel silicium-nitride membraan. De holte wordt gevuld met ontgast ethanol. In de actuator worden de bellen gevormd door gedurende een aantal microseconden een spanningsverschil aan te leggen over het verwarmingselement. Het vermogen dat in pulsen rondom het verwarmingelement wordt gedissipeerd zorgt voor een extreem hoge warmtestroom van enkele honderden $\mathrm{MW} / \mathrm{m}^{2}$ en temperatuur stijgingen van $10^{8} \mathrm{~K} / \mathrm{s}$. Onder deze condities zal spontane nucleatie plaats vinden. Doordat slechts een klein volume wordt verwarmd, zal dit worden gevolgd door een snelle expansie en ineenstorting van de bel.

Informatie over de belvorming en de bellendynamica in de bellen-actuator is verkregen met behulp van stroboscopische fotografie en weerstandsthermometrie gedurende de warmtepuls. De invloed van verschillende operationele parameters en ontwerpvariabelen 
op het moment van nucleatie, de nucleatietemperatuur, de groeisnelheid van de bellen en de belgrootte is bestudeerd. In tegenstelling tot het onderzoek naar de thermische inkjetprinters en andere studies naar belvorming door extreem hoge warmtestromen, worden de bellen in de explosieve micro-bellen actuator gevormd in een gesloten systeem. Om deze reden is ook de invloed van de druk in de holte op de belvorming en de bellen dynamica onderzocht. De resultaten van de experimenten zijn gebruikt om modellen over het warmte transport door het systeem en het effect van een niet-uniforme stroomdichtheid door het verwarmingselement te valideren.

Op het moment dat een bel wordt gevormd zal dit resulteren in een dynamische uitwijking van het membraan. De verplaatsing is gemeten met een laservibrometer. De uitwijking van het membraan wordt niet alleen benvloed door de dynamica van de bellen, maar ook door het ontwerp van de actuator en de druk in de holte. Een constant drukverschil over het membraan leidt tot permanente uitwijking van het membraan. Deze uitwijking kan worden gemeten met een interferrometer. Deze metingen kunnen vervolgens worden gebruikt om het membraan te kalibreren. Na het inklappen van de bel zal het membraan over gaan in een vrije gedempte vibratie en zullen de eigenschappen van het membraan en van de vloeistof de beweging van het membraan bepalen.

De maximale herhalingsfrequentie wordt bereikt wanneer de vorige bel is verdwenen op het moment dat een nieuwe bel gevormd wordt. Bij hogere frequenties ontstaat een permanente bel, die de prestatie van de actuator dramatisch verslechtert. Hoewel het ethanol voor het gebruik wordt ontgast, is er nog steeds een kleine hoeveelheid gas opgelost in de vloeistof. De bel, gevormd door explosieve verdamping, zal niet alleen damp bevatten, maar ook een kleine hoeveelheid gas. Om de bel te laten verdwijnen moet dit gas oplossen in de vloeistof. Dit proces is veel trager dan de condensatie van damp. De hoeveelheid gas in bel zal daarom de tijd bepalen die het kost om een bel te laten verdwijnen. Door een verhoging van de druk neemt de oplosbaarheid van het gas toe en verandert de dynamica van de bellen. De bellen zullen door de hogere druk sneller verdwijnen en de herhalingsfrequentie kan worden verhoogd. Alhoewel de drukverhoging ook resulteert in een kleine afname van de belgrootte en uitwijking van het membraan, verbetert de algehele prestatie significant door de aanzienlijke toename in maximale herhalingsfrequentie.

De beste prestaties die in dit onderzoek zijn bereikt, waren een $2 \mu \mathrm{m}$ dynamische uitwijking voor een herhalingsfrequentie van $5 \mathrm{kHz}$. Voor lage herhalingsfrequenties en omgevingsdruk werd zelfs een veel grotere uitwijking van $6 \mu \mathrm{m}$ en een membraan bewegingssnelheid van $1.3 \mathrm{~m} / \mathrm{s}$ bereikt. Er zijn enkele microJoules nodig om een bel te vormen. Slechts een klein gedeelte van deze thermische e-nergie wordt omgezet in mechanische energie, maar door de hoge energiedichtheid is deze actuator nog steeds erg krachtig. De druk binnenin de bel op het moment dat de bel gevormd wordt, wordt geschat op 25 bar en de groeisnelheid van de bel is een aantal tientallen $\mathrm{m} / \mathrm{s}$. Het feit dat slechts een klein volume verwarmd wordt maakt een snelle ineenstorting van de bel mogelijk. Een vermindering van de hoeveelheid opgelost gas, bijvoorbeeld door een betere ontgassing, zou een herhalingsfrequentie van meer dan $50 \mathrm{kHz}$ mogelijk maken. De explosieve microbellen actuator heeft bewezen dat hij snel is en een aanzienlijke uitwijking kan genereren. Met deze actuator is het echter niet mogelijk om een constante uitwijking te creëren; alleen pulsen kunnen worden opgewekt. Daarom moet men aan toepassingen zoals snelle micro-pompen, micro-compressors en luidsprekers denken. 


\section{Dankwoord}

Dit proefschrift is bijna afgerond en daarmee is ook het einde van mijn promotie in zicht. Voor mij was het een erg leerzame en vooral leuke periode. Een promotieonderzoek doe je natuurlijk niet alleen en de afgelopen vier jaar hebben veel mensen een bijdrage geleverd aan de totstandkoming van dit proefschrift. Graag wil ik dit dankwoord gebruiken om een aantal personen die mij hebben geholpen in de afronding van dit project, in het bijzonder te noemen.

Allereerst wil ik natuurlijk mijn begeleider en promotor Miko Elwenspoek bedanken. Waarschijnlijk ben je een van de weinigen die dit hele boekje gelezen heeft. Alle mooie plaatjes van bellen en meetresultaten in dit proefschrift zou ik niet hebben kunnen maken zonder de hulp van Pino. Verder zijn er nog velen binnen de vakgroep, die mij op de een of andere manier geholpen hebben. Iedereen bij naam noemen zou wat veel werk worden en dan loop ik het gevaar om iemand te vergeten. Daarom wil bij deze alle collega's en ex-collega's van de vakgroep TST, ook wel bekend als MicMec, bedanken.

Naast mijn onderzoek heb ik de afgelopen vier jaar natuurlijk ook de nodige ontspanning gehad. Na het werk een biertje drinken of wat eten in de stad. Doekle, Marcus, Jeroen en Pino, ook na mijn promotie moeten we dit regelmatig blijven doen. Een andere belangrijk vorm van ontspanning is voor mij handballen bij Cabezota. Hierdoor ben ik de afgelopen jaren nog een beetje in conditie gebleven, hoewel daar op woensdagochtend niet altijd evenveel van te merken was.

Natuurlijk wil ik ook mijn moeder en in het speciaal mijn vader bedanken. Vaak herinner ik mij nog het moment dat mijn vader zei: "Ik vind het zo jammer dat ik niet bij jouw promotie zal zijn". Ik weet hoe trots hij nu zou zijn geweest.

Marjolijn, zonder jou zou het allemaal niet zo soepel verlopen zijn. Zeker wanneer ik het wat moeilijker had was jij er altijd met een positieve instelling en om te benadrukken dat ik moest geloven in mijzelf. Ik hou van je! 